\title{
Trans-border Advocacy Network in Tobacco Control: The Adoption of WHO-FCTC Best Practices into Domestic Laws in Ghana and Malawi
}

\author{
Edward Brenya \\ West Virginia University
}

Follow this and additional works at: https://researchrepository.wvu.edu/etd

\footnotetext{
Recommended Citation

Brenya, Edward, "Trans-border Advocacy Network in Tobacco Control: The Adoption of WHO-FCTC Best Practices into Domestic Laws in Ghana and Malawi" (2012). Graduate Theses, Dissertations, and Problem Reports. 4837.

https://researchrepository.wvu.edu/etd/4837

This Dissertation is protected by copyright and/or related rights. It has been brought to you by the The Research Repository @ WVU with permission from the rights-holder(s). You are free to use this Dissertation in any way that is permitted by the copyright and related rights legislation that applies to your use. For other uses you must obtain permission from the rights-holder(s) directly, unless additional rights are indicated by a Creative Commons license in the record and/ or on the work itself. This Dissertation has been accepted for inclusion in WVU Graduate Theses, Dissertations, and Problem Reports collection by an authorized administrator of The Research Repository @ WVU. For more information, please contact researchrepository@mail.wvu.edu.
} 
Trans-border Advocacy Network in Tobacco Control: The Adoption of WHO-FCTC

Best Practices into Domestic Laws in Ghana and Malawi

\title{
Edward Brenya
}

\author{
Dissertation submitted to the \\ Eberly College of Arts and Sciences \\ at West Virginia University \\ in partial fulfillment of the requirements \\ for the degree of
}

Doctor of Philosophy

in

Political Science

\author{
Donley Studlar, Ph.D, Chair \\ Graeme Donovan, Ph.D \\ Scott Crichlow, Ph.D \\ Susan Hunter, Ph.D \\ John Kilwein, Ph.D
}
Department of Political Science
Morgantown, West Virginia
2012

Key words: Tobacco Control, Policy Adoption, Knowledge Transfer, Multi-level Policy Transfer Approaches, Global Advocacy Network, Liberal-Constructivism, Voluntary Agreements, Developing Countries

(C) 2012 Edward Brenya 


\begin{abstract}
Trans-border Advocacy Network in Tobacco Control: The Adoption of WHO-FCTC Best Practices into Domestic Laws in Ghana and Malawi
\end{abstract}

The study investigates the politics characterizing the adoption of tobacco control policy in Ghana and Malawi as a way of contributing to political science literature. It combines the global advocacy network, the multi-level policy transfer, and the liberal-constructivist theories as a single framework to examine their effects on tobacco control policy adoption. Methodologically, the study uses a comparative case study approach complemented by elite interviews, review of secondary materials, and policy documents. The study finds that neither of the Parliaments of the two countries has adopted any legislation that is properly enforced. However, both countries have certain voluntary agreements to control tobacco that were adopted through administrative directives by the Ministry of Health. Ghana has signed and ratified the WHO-FCTC protocol and the voluntary agreements adopted are well respected. In addition, the National Parliament is currently debating a draft tobacco control bill with the hope of passing a law before the end of 2012. On the contrary, Malawi has not signed the FCTC tobacco control protocol and the few voluntary agreements are in jeopardy following the establishment of a privately owned domestic tobacco manufacturing company in 2009. In the end, the study argues that the voluntary agreement often considered ineffective tobacco control instrument in many western countries could be the means of controlling the health hazards associated with tobacco in developing countries where the National Parliament fails to adopt tobacco control legislation. However, the effectiveness of the voluntary agreement depends on the structure and nature of activities of the tobacco industry network and tobacco control network, especially the influence of the Ministry of Health in each of the countries and possibly other developing countries. Where policymakers are directly involve in the tobacco business, little maybe achieve with the adoption of voluntary agreements as tobacco control instruments and vice versa. 
Dedicated to Donley Studlar, Jennifer McIntosh, the late S.W. E. Brenyah, Margaret Osei-Owusu, my wife and children - Patricia Ama Bonsu-Brenya, Edward Nana Kwabena Bonsu Brenya, Jude Nana Adusei Brenya, Angelina Nana Serwaa Brenya-, Judy McGinn and Dick McGinn, Mr. Collins K. Adusei, and His Royal Highness, Otumfuo Osei Tutu II, Asantehene for making this dream a reality. 


\section{ACKNOWLEDGEMENTS}

The contributions and expertise of several individuals and organizations made this dissertation a reality. First, I wish to express my profound gratitude to Dr. Donley Studlar, Dr. Graeme Donovan, Dr. Susan Hunter, Dr. Scott Crichlow, Dr. John Kilwein, and Mrs. Patricia Osei Brenya for the valuable suggestions offered on the earlier drafts of the work, which greatly impacted the quality of the study. In addition, I want to appreciate the financial contributions from the President Office for Social Justice, the Political Science Department, the Eberly College of Arts and Science, and the Africana Studies Program, which made the completion of this study possible.

Other individuals also supported me in diverse ways during this study for which I owe appreciation. In particular, I wish to extend my sincere appreciation for the invaluable love, support and inspiration I enjoyed from my sweetheart, Mrs. Patricia Osei Brenya and children, Edward Brenya, Jude Brenya, and Angelina Brenya, which gave me the peace of mind and motivation to complete the study. In addition, I thank Dr. Dick McGinn and Mrs. Judy McGinn, Ms. Jennifer McIntosh, Mr. Charles Morris and Mrs. Lois Morris, Mrs. Susan Studlar, Mrs. Sharon Mallow, Mrs. LeeAnn Greathouse, the African Students Association, Dr. Francis Godwyll, Mr. Collins K. Adusei, Nana Brefo Boateng, Mr. I. K Gyasi, The Board Members of the Otumfuo Education Fund, Dr. Felix Kumah Abiwu and family, Rev. Kobina Ayittey, Bagnini Kohoun, Kombe Kapatamoyo, Timothy Adivilah, Jacob Sanwidi, Dr. and Mrs. Azumah, Dr. and Mrs. Cwrobreski, Dr. Marty Otanez, Dr. Hadii Mamudu, Dr. Bossman Asare, Dr. Jeffrey Worsham, Dr. Jeffrey Drope, Dr. Sallah, Auntie Cecilia, and His Royal Highness, Otumfuo Osei Tutu II, the King of Asante Kingdom, Ghana, for the enormous moral support and encouragement I received from them.

I also wish thank individuals and institutions such as the Maranatha Evangelistic Ministry Kumasi, the Redeem Christian Church International, Morgantown, the Morgantown Church of Christ, the Prayer Warriors of Benny Hinn Ministries, Rev. Asante Gyimah, Elder Kofi Nti, Pastor Collins, Pastor Nicholas, Mrs. Margaret Osei Owusu, Mr. Samuel Brenyah (B.B), Rev. Evans Brenyah, Godfred Brenyah, Joe Agyeman Prempeh, Michael Brenya, Angelina Brenya, Rexford Brenyah, Mr. and Mrs. Osei Bonsu, Sister Emelia, Kofi Panin, Kofi Ketewa, Pastor Kofi Sarbour and my wife and kids for their spiritual intercession, which granted me the mercies and favor of God for this study to become a reality.

Of course, this study will not have been possible without the encouragement of my late father, Mr. S.W.E. Brenyah, my late uncle Opanin Akwasi Asiama (Sony Boy) and my sweet mother Margaret Osei Owusu, to pursue a doctoral degree and I say a big thank you to them. Last by not the least, I wish to thank the experts/interviewees who willingly accepted to share their expertise on tobacco control with me. Above all, I thank the Lord Almighty for the grace and favor granted me to sail through the doctoral degree which has given birth to this accomplishment. 


\section{Abbreviation and Acronyms}

ACS

$\mathrm{ACF}$

APACT

ATCA

ATCRI

ATSA

BAT

BMRC

CCAP

CDC

CEPS

CHOGM

CIHR

CSR

CTFK

ECLT

FCA

FCP

FCTC

FDA

FORCE

GCTC

GDP

GDHS
America Cancer Society

Advocacy Coalition Framework

Asia Pacific Association for the Control of Tobacco

Africa Tobacco Control Alliance

Africa Tobacco Control Research Initiative

Africa Tobacco Situational Analysis

British American Tobacco Company

British Medical Research Council

Church of Central Africa Presbyterian

Center for Disease Control and Prevention

Customs, Excise, and Preventive Service

Commonwealth Head of Government Meeting

Canadian Institutes of Health Research

Corporate Social Responsibility

Campaign for Tobacco Free Kids

Eliminating Child Labor in Tobacco Growing

Framework Convention Alliance

Framework Convention Parties

Framework Convention on Tobacco Control

Food and Drugs Board

Forum for a Clean Environment

Gold Coast Tobacco Company

Gross Domestic Product

Ghana Demographic Health Survey 


\begin{tabular}{|c|c|}
\hline GES & Ghana Education Service \\
\hline GHS & Ghana Health Service \\
\hline GNA & Ghana News Agency \\
\hline GPRTU & Ghana Private Road Transport Union \\
\hline GTB & Ghana Tourist Board \\
\hline GTC & Global Tobacco Control \\
\hline GTLC & Ghana Tobacco Leaf Company \\
\hline GTSS & Global Tobacco Surveillance System \\
\hline GYTS & Global Youth Tobacco Survey \\
\hline ICITT & International Conference on Illicit Tobacco Trade \\
\hline IDRC & International Development Research Center \\
\hline IGO & International Governmental Organization \\
\hline ILCSC & International Liaison Committee on Smoking Control \\
\hline INGCAT & International Nongovernmental Coalition against Tobacco \\
\hline INWAT & International Network of Women against Tobacco \\
\hline ITG & International Tobacco Ghana \\
\hline ITGA & International Tobacco Growers Association \\
\hline IUAC & International Union against Cancer \\
\hline IUTLD & International Union against Tuberculosis and Lung Disease \\
\hline PAHO & Pan African Health Organization \\
\hline PHASA & Public Health Association of South Africa \\
\hline PATH-Canada & Program for Appropriate Technology in Health, Canada \\
\hline PTC & Pioneer Tobacco Company \\
\hline MDG & Millennium Development Goals \\
\hline $\mathrm{MOH}$ & Ministry of Health \\
\hline
\end{tabular}




\begin{tabular}{ll} 
MOU & Memorandum of Understanding \\
MTC & Meridian Tobacco Company \\
NCS & Norwegian Cancer Society \\
NGO & Nongovernmental Organization \\
NTCSC & National Tobacco Control Steering Committee \\
NYCM & National Youth Council of Malawi \\
RCP & Royal College of Physicians \\
RITC & Research for International Tobacco Control \\
RDD & Research and Development Division \\
SSNIT & Social Security and National Insurance Trust \\
TA & Tobacco Atlas \\
TAMA & Tobacco Association of Malawi \\
TCCP & Tobacco Control Country Profile \\
TOTAWUM & Tobacco Tenants and Allied Workers Union of Malawi \\
UCT & World Health Organization \\
UICC & University of Cape Town \\
UKCR & International Union against Cancer \\
USADF & United Kingdom Cancer Research \\
VALD & Whion for Alternative Development \\
VAT & World Conference on Tobacco or Health \\
WHO & Worled Assembly \\
\hline
\end{tabular}


WHO-TFI WHO-Tobacco Free Initiative

WHO- EMRO WHO- Eastern Mediterranean Regional Office

WHO- AFRO WHO- Regional Office for Africa

YASED Youth Alliance in Social and Economic Development 


\section{Table of Contents}

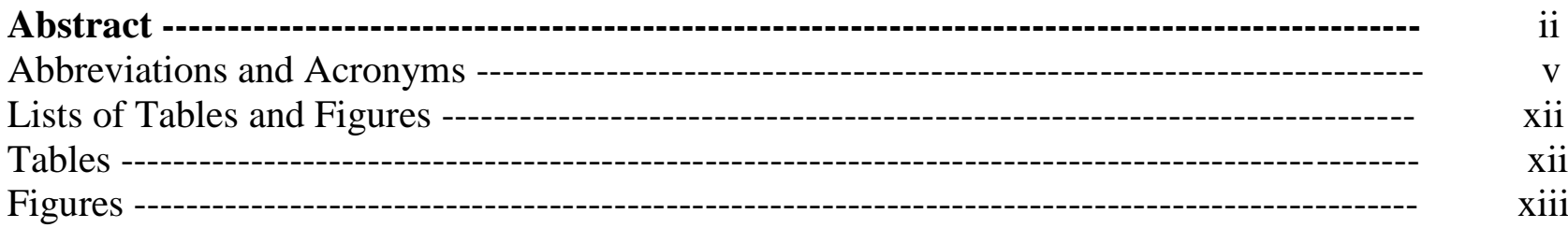

Chapter One: Tobacco Consumption and Control in Developing Countries-------------- 1

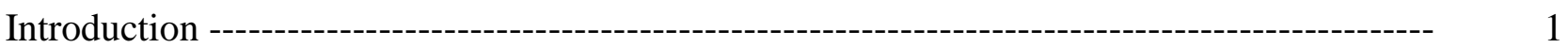

Problem Statement and Purpose of Study------------------------------------------------------ 13

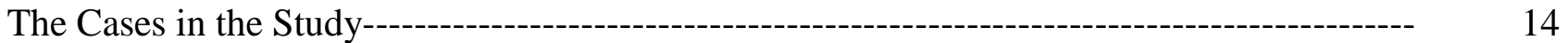

Overview of the Policy Process of Ghana and Malawi---------------------------------------------- 15

The Structure of Bureaucracy and Adoption of Administrative Rules---------------------------- 17

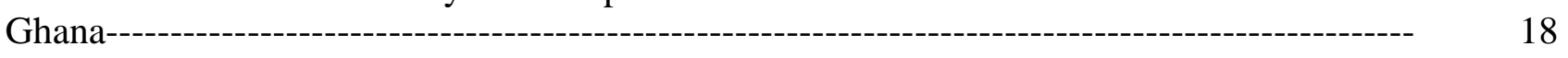

Malawi--------------------------------------------------------------------------------------------------------- 22

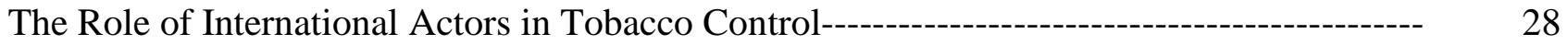

International Research Organizations and Government Agencies--------------------------------- 28

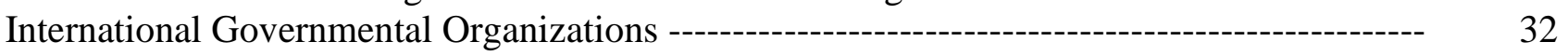

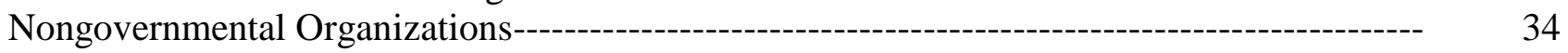

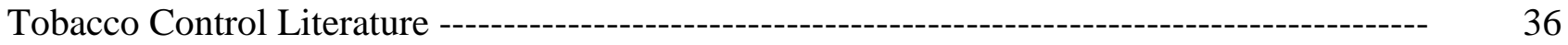

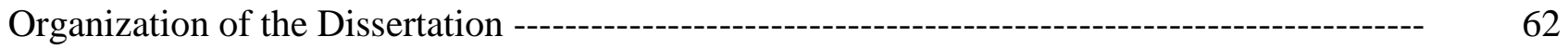

Chapter Two: Theoretical Framework -

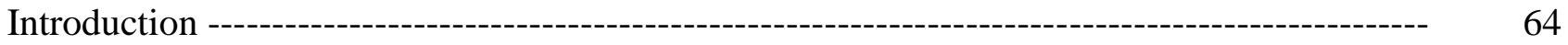

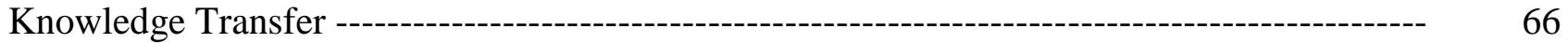

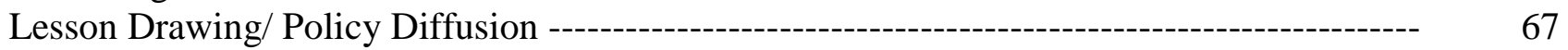

Negotiated / Indirect Coercive Policy Transfer ---------------------------------------------------- 70

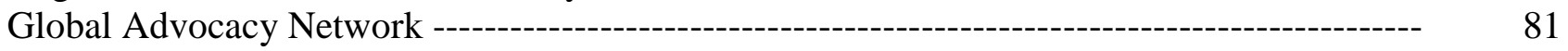

Epistemic Communities ------------------------------------------------------------------------------------ 84

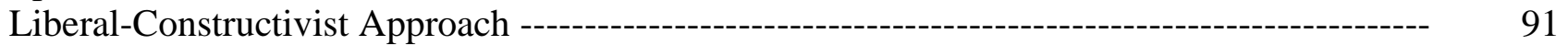

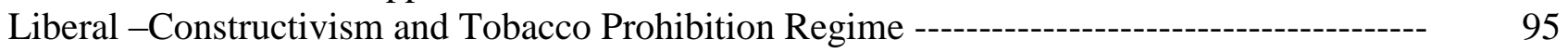

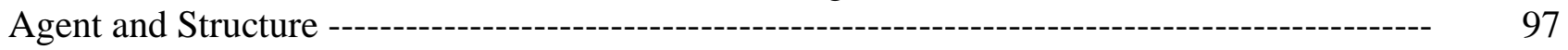

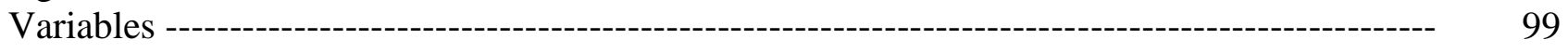

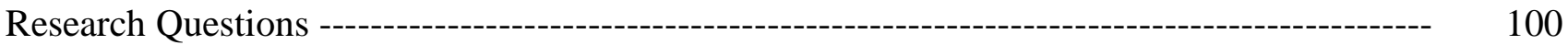

Methodology and Data Collection ------------------------------------------------------------------- 101

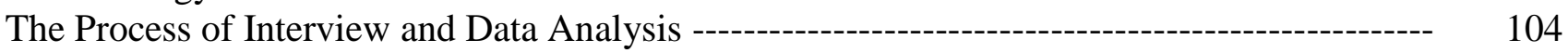

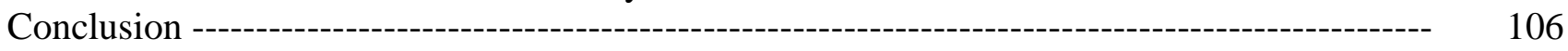

\section{Chapter Three: Nature of Tobacco Control Activities and Policy Change in the}

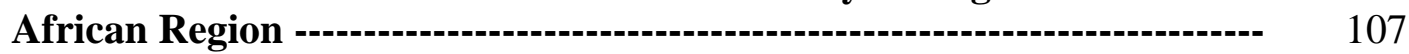

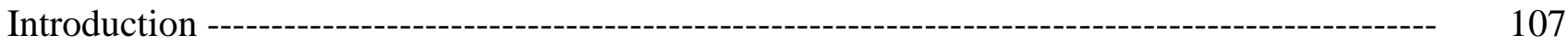

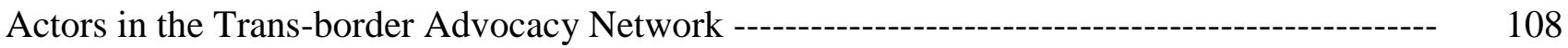

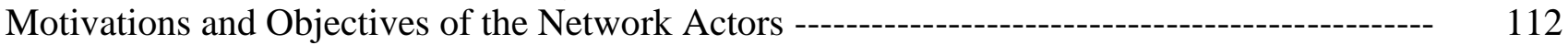


Strategies, Power Structure, and Operations of Network Actors ----------------------------------- 114

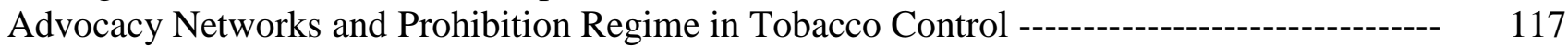

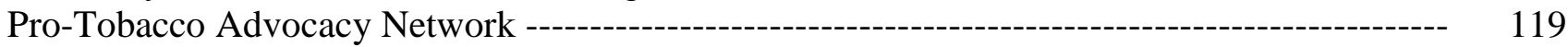

Anti-Tobacco Advocacy Network --------------------------------------------------------------- 123

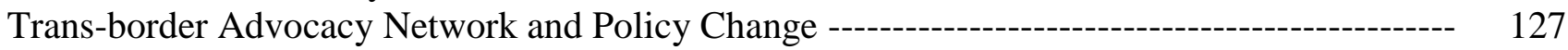

FCTC Instruments and Tobacco Control Policy Change -------------------------------------------- 130

Developed Countries, Global Tobacco Prohibition Regime, and Developing Countries -------- 137

Transnational Actors and Tobacco Control Knowledge Transfer in the African Region -------- 140

Anticipated Tobacco Control Policy Change in the Developing Countries ----------------------- 148

Conclusion ---------------------------------------------------------------------------------------------------- 150

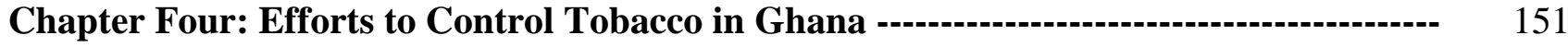

Introduction --------------------------------------------------------------------------------------------------- 151

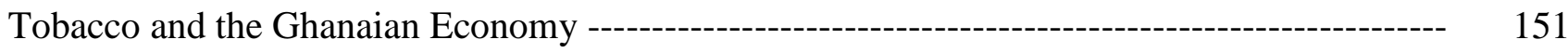

Tobacco Industry Network in Ghana ------------------------------------------------------------------- 153

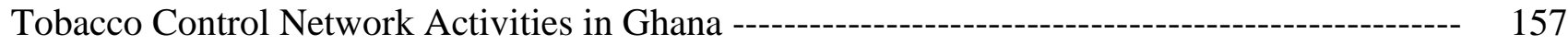

Knowledge Transfer and Tobacco Control in Ghana ------------------------------------------------ 165

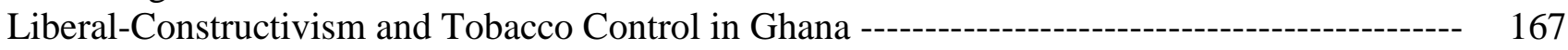

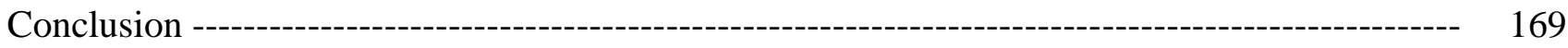

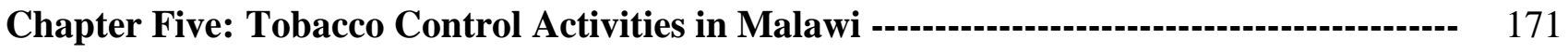

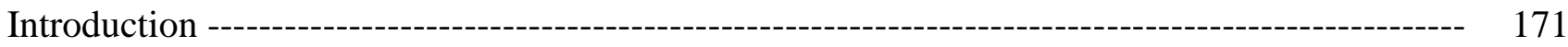

Tobacco and the Economy of Malawi ------------------------------------------------------------- 171

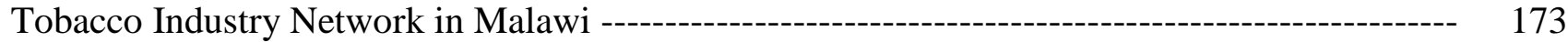

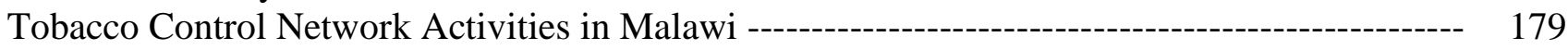

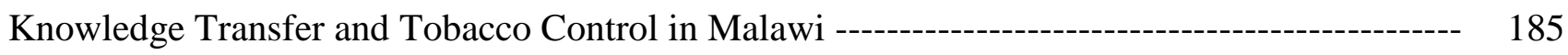

Liberal-Constructivism and Tobacco Control in Malawi -------------------------------------- 188

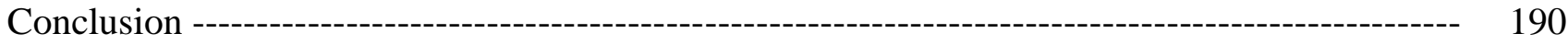

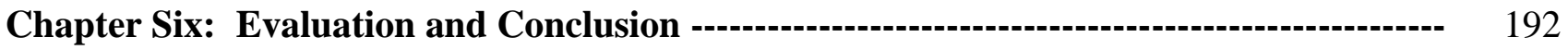

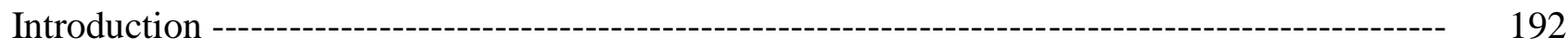

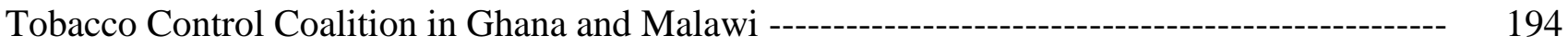

Global Advocacy Network and Tobacco Industry Network ------------------------------------- 195

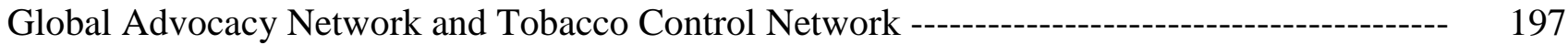

Voluntary Agreements (Tobacco Control Instruments) Adopted in Ghana and Malawi --------- 200

Impact of Voluntary Agreement as Tobacco Control Instrument in Ghana and Malawi --------- 212

Knowledge Transfer and Adoption of Domestic Tobacco Control Policies ----------------------- 213

Liberal - Constructivism and Adoption of Domestic Tobacco Control Policies ------------------ 216

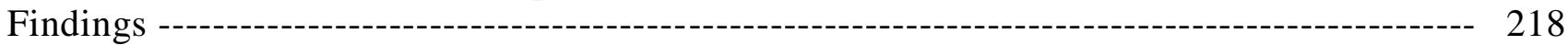

Structure and Influence of Bureaucracy and Voluntary Agreements ---------------------------- 222

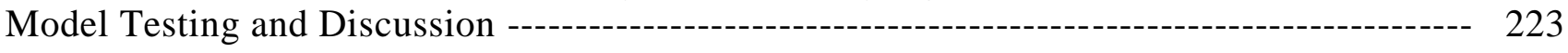




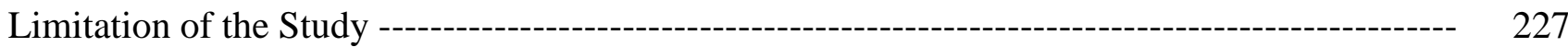

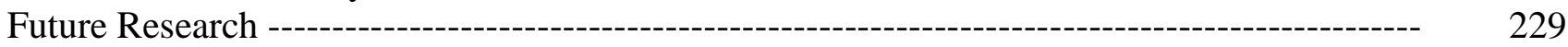

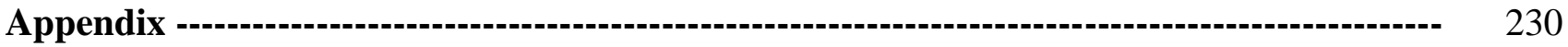

Interview Questions and Protocol ---------------------------------------------------------------------- 230

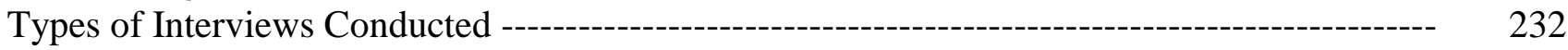

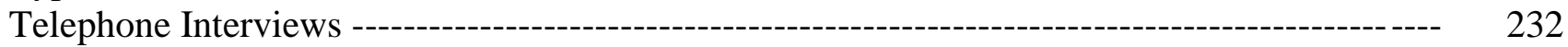

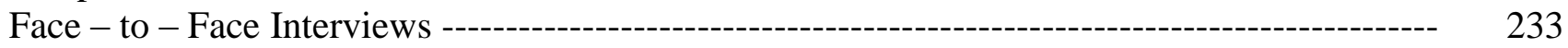

Interviews through Emails ---------------------------------------------------------------------------- 233

List of Individuals and Organizations that Provided Information for the Study ------------------ 234

Copy of Introductory Letter to Interview Respondents Approved by the Institutional Review

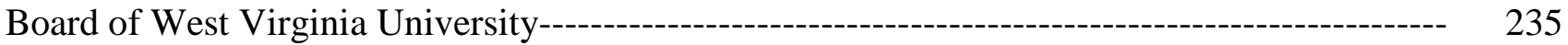

Explanation for Table 6.2: Status of Policies and Progress towards Selected Demand

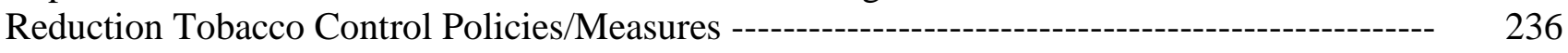

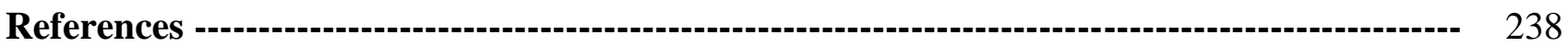




\section{List of Tables and Figures}

\section{Figures}

Figure 1.1 Past and Future Annual Deaths due to Tobacco Use ------------------------------- 2

Figure 1.2 The Four Stages of Tobacco Epidemic Worldwide ------------------------------ 2

Figure 1.3 Composite Diagram of Tobacco Control Policy Transfer ------------------------ 12

Figure 2.1 Knowledge Transfer Diagram ----------------------------------------------------- 66

Figure 2.2 Structure of Activities of Epistemic Communities -------------------------------- 86

Figure 3.1 Trans-border Advocacy Network and Tobacco Control Policy Change ------- 130

Figure 6.2 Sample Health Warning on Cigarette Packs and Point of Sale in Ghana ------- 205 


\section{Tables}

Table 1.1: $\quad$ Timeline of Tobacco Production, Manufacturing, and Control Efforts

(including the Adoption of Voluntary Agreements) in Ghana --------------------- 21

Table 1.2: $\quad$ Timeline of Tobacco Production and Control Efforts (including the Adoption of

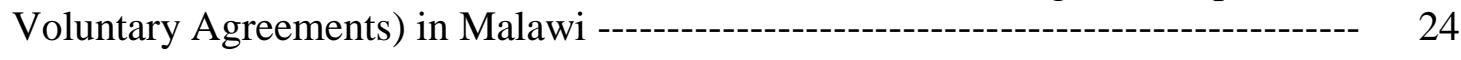

Table 1.3 Prevalence and Stages of Tobacco Epidemic among Developing Countries ------- 28

Table 1.4 Characteristics of the Stages of the Tobacco Epidemic ------------------------------- 29

Table 1.5 Relevance of Tobacco Control Policies of Different Income Levels ---------------- 55

Table 4.1 Composition of the National Tobacco Control Steering Committee of Ghana ----- 158

Table 6.1 Status of the Policies and Progress towards Selected Demand Reduction Tobacco

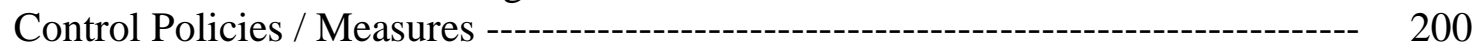




\section{Chapter One}

\section{Tobacco Consumption and Control in Developing Countries}

\section{Introduction}

The battle over the adoption of relevant policies to control the consumption of tobacco products has been a major issue confronting all developing countries, including Ghana and Malawi. Since the 1970s when countries in the developed world started to adopt and enforce stricter tobacco control policies, many tobacco companies have relocated to the developing countries. This has resulted in a rapid increase of tobacco consumption and its related health hazards in the developing countries. The World Health Organization $(1997,2008)$ and World Bank $(1995,1999)$ estimate that there will be an estimated 500 million total tobacco-related deaths by the year 2030, and that $70 \%$ of the deaths will be from the developing countries if the trend of tobacco consumption does not change ( see Figure 1.1). In attempt to prevent such tobacco epidemic, some organizations and individuals have been engaging in activities that transferred tobacco control knowledge and strategies to the developing countries (Cairney et al 2012).

The action of these actors is informed by the experience of the developed countries and the knowledge that the anticipated deaths and epidemic caused by tobacco consumption will decline if the developing countries adopt relevant tobacco control policies (WCTOH 2000; Lopez et al 1994, also see Figure 1.2). However, the tobacco industry network and their affiliates have been employing strategies and politics to prevent the adoption of control measures that can hamper their activities in the developing countries. The strategies include but are not limited to lobbying influential policymakers to prevent the adoption of restrictive tobacco control laws and projecting tobacco as an important economic commodity for the development of the countries where the companies operate (WHO 2004; Mamudu et al 2008). 


\section{Figure 1.1 Past and Future Annual Deaths due to Tobacco Use}

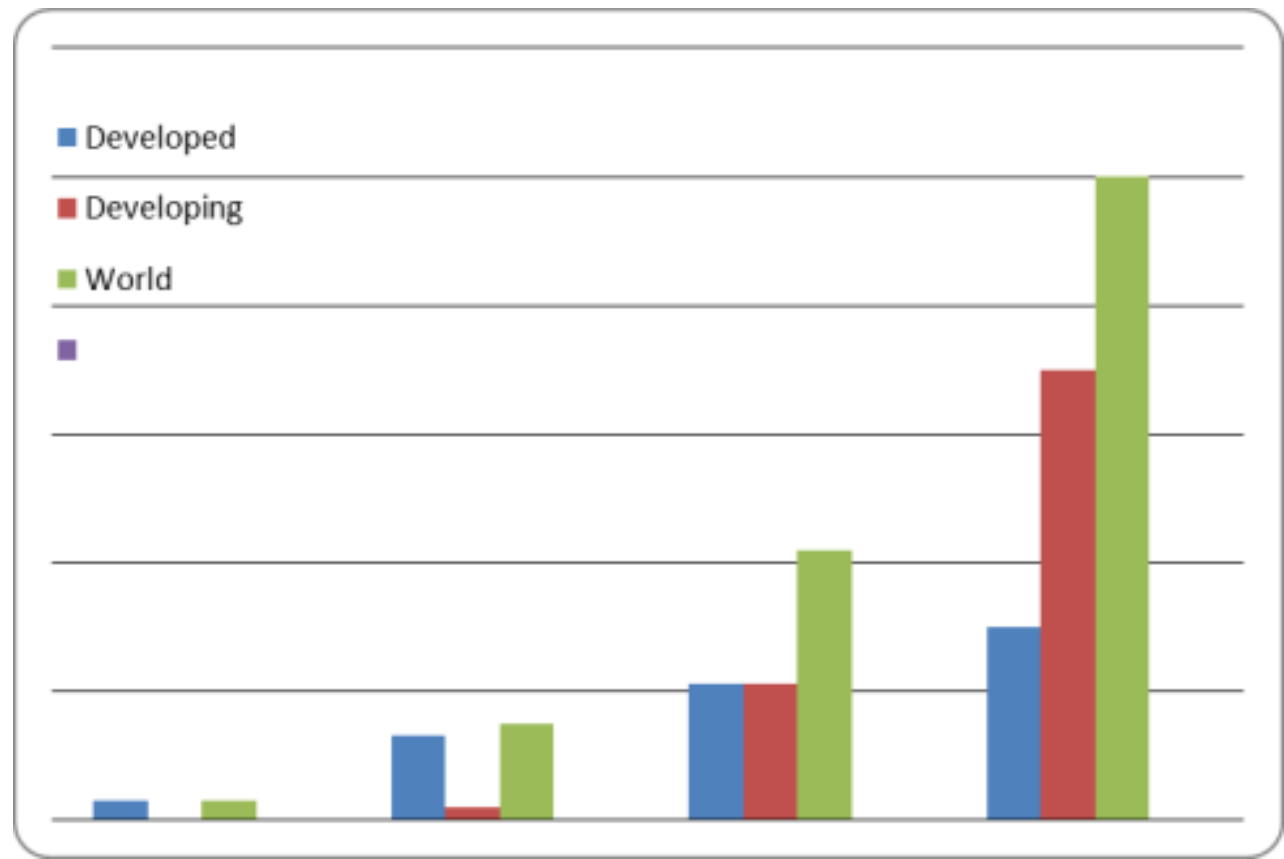

Source: WHO (2002) The Tobacco Atlas, p.36. Scale: $1=50$ million deaths

\section{Figure 1.2: $\quad$ The Four Stages of Tobacco Epidemic Worldwide}

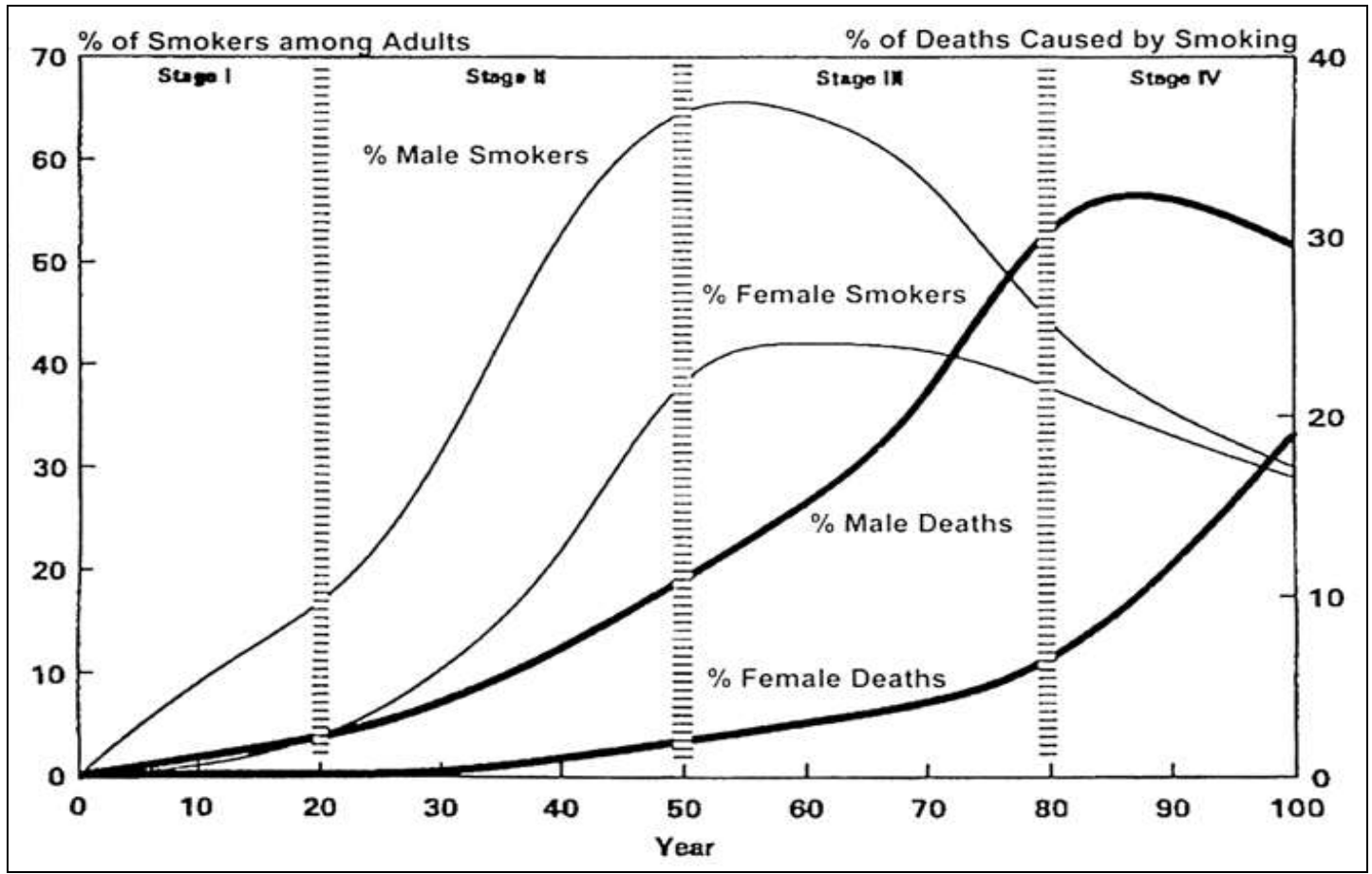

Source: Lopez AD, Collishaw NE, and Piha T. (1994). 
The tobacco companies are also alleged to offer funding and scholarships to the youths to study at higher institutions while at the same time enticing them to smoke cigarettes as means of getting the youths hooked to the products at early age (Barraclough and Morrow 2008). The companies also promote the growing of tobacco leaves in the tobacco producing countries and sponsor major countrywide events where key government officials are invited to present the awards (WHO 2004, WHO 2008). Furthermore, the companies use public relations and the manipulation of the media to project a favorable image of their activities (WHO 2004; IDRC 1995; Interview 2011). Therefore, the tobacco pandemic facilitated by the activities of the companies has evolved into an international public health issue because of the extent of reach of the tobacco companies globally.

In response to the threat posed by a global tobacco epidemic, the World Health Organization (WHO) exercised the power granted it in Article 19 (2) of the Constitution of the WHO by the World Health Assembly (WHA), - the highest decision making body of the WHO - to adopt standards, legislations, conventions, and agreements to promote and protect international public health (WHO 2009). The World Health Organization established the Framework Convention on Tobacco Control (FCTC) in 2003 as the first international health treaty to control the consumption of tobacco products and the health hazards affiliated with tobacco use (Mamudu 2005; 2008; WHO 2009). The preamble of the FCTC treaty states that the special contributions and participation of NGOs and other entities not affiliated with the tobacco industry is essential to achieving the objectives of the convention at both the country and international levels (MOH, GHS \& FCTC 2010; WHO-FCTC 2009).

Some nongovernmental organizations (NGOs) and governmental organizations (IGOs) based in the U.S, Canada, and the U.K have been contacting and transferring knowledge of tobacco control to Ghana and Malawi, two of the developing countries that are yet to adopt comprehensive domestic tobacco laws. The knowledge transfer agents include the government officials from these developed countries, international governmental organizations like the Commonwealth of former British colonies, 
and the World Health Organization, NGOs like the Bloomberg Foundation and the American Cancer Society (ACS), and certain tobacco control epistemic communities. These contacts are mostly made through formal meetings of the IGOs such as meetings of the Commonwealth countries, conferences of the WHO, World Conference on Tobacco or Health (WCTOH) and personal visits, while others use informal means such as correspondence or communication through the internet.

The contacts are mostly facilitated by the desire of the transnational actors to prevent a tobacco epidemic in the developing countries and also to socialize the countries into the community of countries with effective tobacco control policies (Cairney et al 2012). Generally, the actors recommend tobacco control measures/strategies to the developing countries. Mostly, the strategies and practices recommended by the knowledge transfer actors have been tested and found to be effective measures for controlling the consumption of tobacco products and its hazards in the countries they were earlier applied. Therefore, the measures are considered "best practices" for addressing the tobacco issue that could be introduced to other countries (CDC 1999; WHO 2008; Laugesen et al 2000).

Mostly, the actors use education on the health hazards of tobacco to encourage the developing countries to adopt the best practices and ideas as domestic law with the hope that the adoption will generate similar result as the country where the practices were initially adopted. In some instances, the actors use incentives to encourage the developing countries to adopt the tobacco control policies. The use of incentives is considered as an indirect coercive measure to get the countries to adopt tobacco control policies. However, the use of incentive is less coercive than regulatory measures that use command and control to promote some policy options and interest.

This study seeks to understand the political struggles and societal dynamics during the transfer of knowledge leading to the adoption of the best practices into domestic tobacco control laws. In other words, it investigates the processes through which the ideas and strategies recommended by the tobacco control agents are adopted as domestic laws in the developing countries. Specifically, it examines the 
process of policy change through the lens of domestic laws being adopted in Ghana and Malawi - both member countries of the Commonwealth in sub-Saharan Africa. In addition, it examines the changes in the behavior of the developing countries that are indicative of the changes in the policy position.

Often, the notion of policy change when simply considered connotes "the movement of policy over time" (Studlar 2009:73). In this sense, policy change can take the form of moving from a weak policy to adopting more stringent policies. This could happen through the domestic development of policy by policymakers or policy diffusion. However, the study moves beyond the simple conception of policy change and adopt a view that enables it track and analyzes major changes in the policy behavior of the countries. The initial aim of the study was to examine the tobacco laws passed by Parliament that are properly enforced in both countries as were done by Asare (2007) and Studlar (2002, 2004). However, with the absence of such legislation in both countries, this study was compelled to adopt a broader view of policy adoption that enable it to examine policy change in the countries by focusing on the voluntary tobacco control agreements, changes in the rhetoric of policymakers, or the adoption of policies that indirectly satisfy the spirit of the tobacco control measures. Often voluntary agreements to control tobacco have been considered as a weak tobacco control instrument and this study seek to ascertain if that is the case in the developing countries.

The voluntary agreement are British derived means of policy implementation whereby the actors to be impacted by a policy instrument are engaged in negotiation on the adoption of the instrument to ensure compliance during implementation (Vogel and Kagan, 2004). Considering that the voluntary agreements are spearheaded and adopted by the Ministries of Health - a bureaucratic agency the studies also examine how the structure and influence of bureaucratic agencies also impact the adoption of tobacco control instruments. This is an exploratory study that seeks to analyze the policy process, especially the interactions between the external and internal factors, pro and anti- tobacco interests, and the adoption of tobacco control policy in the two countries, in detail, applying the existing 
theories. The overall research question focuses on understanding how and why certain tobacco control policies are adopted in the developing countries. The idea is to examine the determinants of policy based on the belief that policy development is an outcome of the interplay between transnational and domestic actors and factors (Cairney et al 2012). Therefore, this study uses an approach that combines three theories of policy transfer and change processes - the multi-level policy transfer approaches, the global advocacy network, and the liberal-constructivist framework of international relations - to understand the politics of policy adoption.

The multi-level policy transfer approaches explains policy adoption motivated by both domestic and external factors and pressures as well as those that are coerced or done voluntarily. Therefore, the study investigates the tobacco control policies or voluntary agreements that have been adopted in the two countries to ascertain if the adopted policies/measures were influenced by pressure from domestic and external tobacco control actors. Specifically, the theory investigates how governmental institutions can be influenced by voluntary and indirect coercive means involving incentives to adopt certain tobacco control instruments. Moreover, it examines how policy borrowing from countries with tested and better tobacco control practices, especially those promoted by the transnational tobacco control actors, influence policy adoption. The paper also investigates laws that have been adopted by the countries that indirectly address some of the concerns of the tobacco issue in addition to the direct tobacco control instruments. In addition, it investigates the effectiveness of policy adoption that is indirectly coerced through the offer of economic and other incentives. The interactions of the knowledge transfer agents with actors of the developing countries at meetings and conferences of international governmental organizations play a significant role in the knowledge transfer process. Therefore, the study also investigates how the membership of IGOs such as the Commonwealth and WHO impact the adoption of tobacco control laws through the lens of the multi-level policy transfer approaches. 
This study also expects that the global advocacy network theory will help to explain how some governments, NGOs, international governmental organizations (IGOs), and epistemic communities who share similar values collaborate to influence policy change globally. For instance, policy adoption or non-adoption in the area of tobacco control is influenced by the actions of the two major advocacy networks in the tobacco control policy subsystems - the pro-tobacco advocacy network and antitobacco advocacy network (Farquharson, 2003). Therefore, the global advocacy network approach is used to illuminate the political struggles between the networks and how it impacts the adoption of tobacco control laws in Ghana and Malawi. The pro-tobacco advocacy network is made up of actors who are connected by the values and interests of the tobacco industry such as the tobacco manufacturing and leaf buying companies, farmers, importers and distributors, retailers, and employee associations.

The network projects tobacco as the economic pillars of tobacco economies and encourage them not to adopt tobacco control policies that will decrease the contribution of tobacco to their GDP. On the other hand, the anti-tobacco advocacy network members are bonded by their mutual interest in prohibiting the consumption of the tobacco products and its related health hazards. They represent collaboration between governmental and nongovernmental actors as well as relevant epistemic communities who are promoting the adoption of their interest as tobacco control ideas in the developing countries. Generally, the network promotes the signing and ratification of the FCTC protocol with the assumption that the signing of the protocol will motivate the adoption of their interest and ideas presented as effective tobacco control measures as domestic laws to address the tobacco related hazards.

Therefore, the global advocacy network theory is used to assess the strategies used by the tobacco industry network to prevent the adoption of effective tobacco control measures in the adopting countries and how successful they are with the blocking strategies. It investigates if the tobacco 
industry network succeeds in the attempt to prevent tobacco economies from adopting effective tobacco control policies or not. Furthermore, the role undertaken by actors from the US, Canada, U.K and other leading Commonwealth countries as well as the WHO and other domestic actors (governmental, NGOs and advocacy groups) to halt the activities of tobacco industry network in the developing countries by getting governmental institutions such as the Ministry of Health to adopt tobacco control instruments is examined under the global advocacy network theory. In that sense, it examines how incentives and voluntary means are used to motivate the adoption of tobacco control measures. It also investigates if the signing and adoption of the FCTC protocol necessarily influence the adoption of effective tobacco control laws.

Given that the tobacco control activities are carried out by both coalitions of NGOs and IGOs, including regional IGOs such as the African Union (AU) and countries like South Africa as well as individual actors and organizations as part of the coalition, the study investigates if the knowledge transfer is successful when it is disseminated by a coalition or by the individual actors and organizations. In that regard, it examines the manner through which the tobacco control knowledge and tactics are transferred to ascertain if the transfer agents act independently or in coalitions. It also determines if the actors in the leading tobacco control countries target specific developing countries or groups of countries, whether they transfer the same knowledge to all the countries they contact, and if the tobacco control instruments being transferred is based on the FCTC recommended measures or deviates from them.

It explores the different actors involve in a coalitions when the contacts are made through coalitions, the venues where the contacts take place and the factors that determine which country to contact. The study also investigates the possible partnerships used by the actors to transfer the tobacco control knowledge under the same theory. The partnership can take the form of the US government partnering with Canada, U.K, the Commonwealth Secretariat, the WHO, the NGOs, or epistemic 
communities to transfer knowledge on tobacco control to the adopting countries. It also examine if there are other possible partnerships in the network among sub-groups of the epistemic communities, NGOs and/or IGOs.

While the global advocacy network examines the collaborations and strategies adopted by the two networks to influence the adoption of tobacco control policies, the liberal-constructivist approach looks at how socially constructed ideas and relevant information are used to influence the actions and understanding of people, especially policy actors, in such a way that they change their actions and perceptions on issues and adopt policy changes (Goldstein and Keohane 1993; McNamara 1998; Mamudu 2005; Wendt 1992; Finnemore 1996). With this theory, the study examines how the transnational tobacco control actors are using the transmission of the new tobacco control ideas to the developing countries to enforce compliance with the FCTC norms. Importantly, it investigates why the receiving countries adopt or do not adopt the tobacco control ideas disseminated by the tobacco control agents. In addition, it investigates how the dissemination of relevant information on tobacco control is changing the understanding/perception of people about tobacco issues in the receiving countries. In this sense, the study examines how the people considered the tobacco issue in the past and how they consider it now as well as the factors that account for the changes in perception. Additionally, the study examines the possible changes in the actions, opinion, rhetoric, understanding or perception of the people and policy makers on the tobacco issue as well as the support of the people for adopting effective tobacco control laws.

This study presumes that the examination of the changes in actions, perceptions, rhetoric and the adoption of policies that directly or indirectly address problem of tobacco in these countries will help to illuminates the inherent political struggle underlying the processes of policy change. The adoption of the broader view of policy change will contradict other studies whereby voluntary agreements on tobacco control were not considered as effective variables for tracking the changes in 
tobacco control policy (Studlar 2004; Asare 2007; 2009). However, it is similar to the study of Drope (2010) in which the author examined the adoption of smoke free policies in certain African countries, using alternative tobacco control arrangements such as voluntary agreements as variables of analysis because of the absence of smoke free laws adopted by the National Parliaments . This study anticipates that using the voluntary agreements comprising administrative directives to analyze the tobacco control policy adoption is a valid measure because the FCTC treaty calls for the adoption of laws, regulations, administrative directives, and other tobacco control measures to promote the treaty (FCTC 2009; MOH, GHS \& FCTC 2010).

By combining all three approaches within a single framework of analysis, the study hopes to provide a comprehensive overview of policy change. The intention is to address the problem of weak understanding of policy change in the literature of political science, comparative public policy and politics, and international relations resulting from the overreliance on only one of these approaches to explain policy change. Notable exceptions to the general weak understanding are the studies of Keck and Sikkink (1998) and Mamudu (2005), which combined at least two of these approaches within a single framework of analysis. Both Keck and Sikkink (1998) and Mamudu (2005) use the liberalconstructivist and the transnational advocacy network theories in their studies. Keck and Sikkink (1998) examine how transnational actors involving some IGOs, NGOs, and governmental actors collaborate to influence countries to adopt certain preferred policies. They applied their theory to the environment, human rights, and women rights to ascertain how the network of actors was able to influence the adoption of domestic policies on those issues.

One weakness of the study of Keck and Sikkink (1998) is that it leaves out the epistemic communities who are mostly influential in generating ideas that creates policy change. Epistemic communities are groups of experts who are bonded by their shared beliefs, competence, and expertise on certain issues, and the desire to influence policy related to those issues (Haas 1992). Therefore, the 
analysis of the activities of epistemic communities is vital to an elaborate understanding of how authoritative knowledge and critical ideas influence policy change. The attempt of Farquharson (2003) to address the weakness of the study of Keck and Sikkink by incorporating the epistemic communities' theory into her study was limited by its focus on only Australia- a developed country. While this was not the objective of the study, it did not provide an understanding on how the theories compare in two or more countries. Her study also did not include the wider context of the liberal-constructivist approach within the tobacco control policy subsystem. The study that came closest to addressing all the concerns raised above was that of Mamudu (2005). Unfortunately, the study examined only why the WHO-FCTC emerged as a global tobacco control regime and did not investigate how the regime influences countries to adopt domestic tobacco control laws.

This research moves beyond Mamudu (2005) to study empirically how the tobacco control regime already in place is influencing policy change in selected developing countries through transfer of knowledge, which leads to the adoption of new domestic tobacco control policies in the countries. It relies on case studies focusing on the process of knowledge transfer of tested and better practices of tobacco control by the officials of the U.K, U.S, Canada, WHO, the Commonwealth of former British colonies, some NGOs, and epistemic communities based in these countries (referred to as developed countries) to selected developing countries in the African region: Malawi and Ghana (referred to as developing countries) ${ }^{1}$, which lead to the adoption of domestic laws (see Figure 1.3). Therefore, this study is different from other studies that mainly focus on the processes of tobacco control in the developed countries.

\footnotetext{
${ }^{1}$ For the justification for using these two countries refer to the section under the cases in the study.
} 
Figure 1.3: $\quad$ Composite Diagram of Tobacco Control Policy Transfer

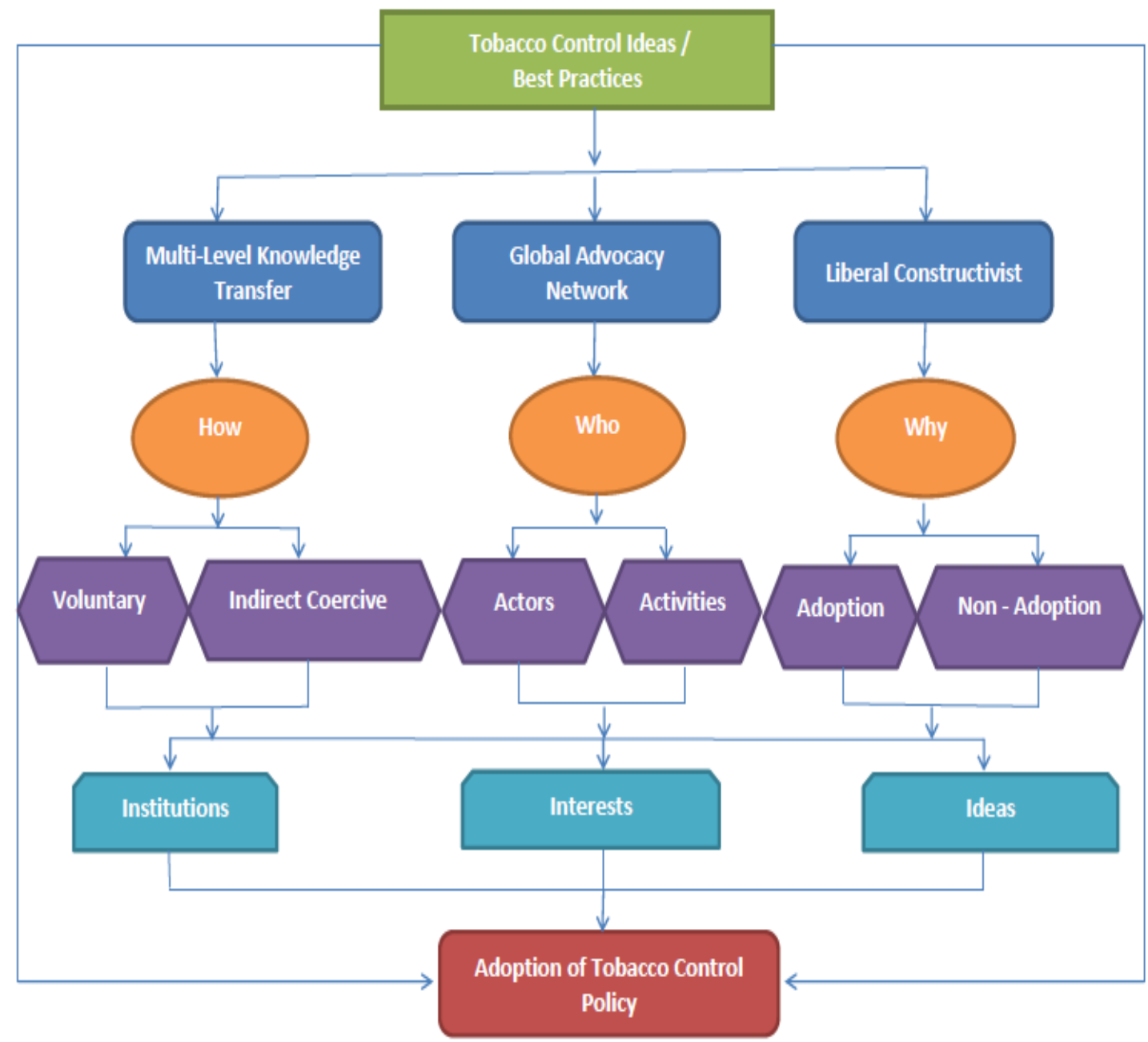

Source: (Self Developed Model Based on the Studies of Farquharson 2003, Finnemore 1996, Mamudu 2005, Dolowitz and Marsh 2000, WHO 2008, RITC 2006, PAHO 2003, PHASA 2007, Evans and Davies 1999, Evans 2004, Ladi 2005, Wendt 1992, Gidden 1996)

Methodologically, the study adopts a comparative case studies technique that relies on interviews of elites and a review of policy documents and periodic reports on the tobacco control of countries submitted to the WHO-FCTC, the reports by the World Bank, and the American Cancer Society on the tobacco control activities and policies of the countries. It also reviews the reports and materials from conferences, seminars, workshops, and media reports on meetings and tobacco control activities involving the officials of the developed and developing countries as well as the policy statements of governmental officials of the countries. It uses a triangulation approach whereby the 
primary interviews are combined with existing secondary material to both complement and assess the authenticity of the information received.

The diagram above summarizes the way this study perceives the nature of tobacco control knowledge transfer. It postulates that tobacco policy transfer occurs through the dissemination of tobacco control ideas and best practices to the adopting countries. The multi-level knowledge transfer approaches explains how voluntary and indirect coercive - incentive - are being implored to engage bureaucratic institutions and interest groups to promote ideas for controlling tobacco. The global advocacy network helps to identify and explain the activities of the tobacco control actors who are promoting their interest as tobacco control ideas for adoption. Lastly, the liberal-constructivist approach explains why the ideas being disseminated are being adopted or not adopted at the receiving countries.

\section{Problem Statement and Purpose of Study}

Scholars of policy transfer have argued that jurisdictions and countries confronting similar public policy problems can draw lessons to address their domestic problems from countries that had already addressed similar issues (Rose 2005). Public health and political science scholars have also identified the tobacco pandemic as a global epidemic confronting all countries, both developed and developing. WHO (2008) has established that the tobacco companies adopt similar tactics such as using deceptive advertising to attract new users in all countries. Moreover, it has been argued that many developed countries have succeeded with the adoption of effective control measures that have decreased consumption of tobacco. For instance, Warner (2000:418) argues that the total consumption of cigarettes in the US decreased from 633 billion in 1981 to 479 billion in 1997 because of the adoption of tobacco control measures.

There are reports indicating that some governmental and nongovernmental actors from developed countries are contacting developing countries to influence the adoption of domestic tobacco 
control laws (PAHO 2003; PHASA 2007; WHO- EMRO 2001; WHO-AFRO 2000; WHO 2005; RITC $2006)^{2}$. Unfortunately, these reports generally do not illuminate the political struggles that occur during the process of knowledge transfer, and the subsequent adoption or non-adoption of domestic laws in the receiving countries. For instance, the reports of the WHO on conferences and training sessions on tobacco control for some developing countries fall short of in-depth theoretical and academic analysis examining if the sessions generate policy adoption. On the other hand, the periodic reports by the World Bank, American Cancer Society and the FCTC, mostly indicate the tobacco policies adopted by individual countries without analyzing the process that led to the adoption of the policies. This study builds on such reports and undertakes an in-depth academic investigation of the types of knowledge being transferred, the policies that are adopted as a result, and the political struggles that occur between the anti and pro-tobacco advocacy networks during the process.

\section{The Cases in the Study}

The core objective of this research is to examine the best practices of tobacco control policies adopted into domestic laws by Ghana and Malawi, and the political struggles that occur during the adoption processes. The Parliaments of the two countries selected for analysis have not adopted any comprehensive tobacco control legislation that is effectively implemented. However, each of the countries has certain voluntary agreements on tobacco control instituted through the directives of the Ministry of Health and other significant governmental ministries and agencies (see Table 1.1). Generally, the voluntary agreements do not have the full force of the laws and legal backings because they were not passed by the Parliaments of the countries. However, they are greatly respected by the majority of the public and the stakeholders of the tobacco industry, especially in Ghana because the tobacco interest groups were involve in the adoption of the instruments.

\footnotetext{
${ }^{2}$ These reports are reviewed in detail at the literature review section
} 


\section{Overview of the Policy Process of Ghana and Malawi}

Ghana and Malawi have unitary systems of government, where the National Parliament is the official law making body. The structure of policy making in both countries is such that the Cabinet recommends policy proposals that are legislated into laws by the Parliament. The Cabinet comprise the ministers of substantive ministry, the Vice President and the President and it serves as the highest decision making body of the executive arm of government. The Cabinet recommends policies to the Parliament for consideration and passage into law to ensure consistency of the policy with the agenda of the government. Normally, cabinet meetings are chaired by the President or the Vice President. Additionally, administrative agencies and departments also have power to issue administrative and executive directives or to promulgate rules but the directives and rules are mostly persuasive and not properly enforced and legally binding as laws passed by the National Parliament.

Mostly, the executive and administrative agencies adopt administrative directives to address issues of concern when the National Parliament fails to pass legislation or the Cabinet does not introduce a bill to the Parliament for legislation to address the problem. In view of the concentration of law making power in the central government, which is exercised by the Parliament or executive agencies and departments, the laws of each of the countries are fairly uniform countrywide, as usually the case with unitary systems of government (Cairney et al 2012). However, the media and vocal civil society and interest groups have great influence in the policy making because of how they shape public opinion on the activities of the government, which directly or indirectly impact the political capital of both the President and the members of Parliament.

In Ghana, the standing orders and procedure for the introduction of bills to Parliament allows both private individuals and the government to introduce bill(s) to Parliament (Interview 2011). Bills introduced by individuals, including Members of Parliament, are referred to as a private member motion. However, cabinet ministers have always introduced bills to Parliament and it has now become 
an unwritten norm that private members, including Members of Parliament, present their policy proposal to relevant sector ministry for consideration and subsequent introduction to Parliament (Interview 2011). Nonetheless, members of Parliament are able to add their input to a policy through amendments at both the committee and general assembly levels. Normally, all policy proposals have to be cleared by the Cabinet before they can be introduced to Parliament. As a result, the Attorney General Department has become a clearing house for all policy proposals before they are forwarded to the Cabinet to decrease the case load of the Cabinet.

The media and vocal civil society groups are able to influence policy at both the Cabinet and Parliament because of their influence on the political capital of the elected officials. The President is elected by the entire country and the winner must obtain $50 \%$ plus 1 of the valid votes. On the other hand, members of Parliament are elected separately on a system of voting whereby the candidate that obtains the simple majority votes becomes the winner. Therefore, the elected officials have both collective and individual responsibility and accountability to their constituents and are mindful of protecting their political capital. Even though, members of Parliament mostly tow the party line in their voting, some of them use amendments to shape the policy to reflect the interest of their constituents. In addition, sector ministries and government agencies also use executive and administrative directives to promote policy objectives in instances where the Cabinet does not show interest in introducing a bill to Parliament for a legislation to address the issue of concern. Normally, the directives reflect outcome of negotiation with relevant actors to be affected by the directives who have voluntarily agreed to respect the directives.

The story is not different for Malawi as the policy agenda is set by the President whose decisions are made in conjunction with the members of the Cabinet. The Cabinet is made up of Ministers of substantive government ministries, the Vice President and the President. Policy proposals to be legislated into laws are introduced to the chamber by a Cabinet level Minister. However, 
individual members of Parliament can sponsor and introduce bills in the Parliament on Thursdays (Interview 2011). Malawi has established a Law Commission since 2004 to which special interest groups and private individuals can apply directly to consider drafting legislation for consideration of the National Assembly (Makoka et al 2011). The Commission is a semi-autonomous body under the Ministry of Justice and members are selected from a wide range of interest groups known to be opened to well researched and evidence-backed arguments that can be represented by legal opinion. The media and vocal interest groups play influential role on the adoption of policy proposals through their leverage on the elected officials who act to protect their political capital just like Ghana.

Malawi also has a voting system where the President is elected by the whole nation and the Members of Parliament are elected by individual constituencies. Therefore, the elected officials are careful to gain a favorable approval rating from the public, which makes the media and other civil society organizations to be influential to the policy making process. In addition, the administrative agencies and governmental departments also have the power to issue directives or to promulgate a rule to address certain issues under their jurisdiction when the Parliament fails to do so. Like Ghana, administrative directives issued by sector ministries of Malawi are normally an outcome of voluntary agreement between the ministry and the groups to be affected by the directives.

\section{Structure of Bureaucracy and the Adoption of Administrative Rules}

Both Ghana and Malawi have unitary systems of government with large bureaucracies that play a major role in the administration of the countries. The bureaucrats are career civil servants who serve in their position for a long time and get promoted to higher positions through the completion of requisite examinations. The bureaucrats oversee the daily administration of the affairs of the government and also see to the implementation of laws passed by politicians and their own rules and regulations. Because of the political history of instability of governments of these countries in the past, 
the bureaucracies tend to be the agent of continuity and are often in charge of the day to day administration of the countries. Even after the re-introduction of multi-party elections, the bureaucrats still play the dominant role of overseeing the day to day administration of government affairs because the political appointees of the elected government who oversee the realization of its agenda often stay for shorter period of about 22 months or more if the government is re-elected for another four year term. The bureaucratic agencies have branches in the regional, district, and local levels that help to implement policies of the government, but all of the branches are controlled vertically from the national level. The bureaucrats often serve in their roles for a long time and are often more experienced than the elected officials on how to effectively get preferred policies implemented. Mostly, the bureaucratic agencies are more comfortable with using their administrative rules to address public problems than laws enacted by the elected officials, which are sometimes considered ineffective because of the various interests that the politicians have to please when enacting laws.

\section{Ghana}

Ghana is an independent republic and was the first country in sub Saharan Africa to obtain independence from the British in 1957. The country is located in West Africa bordered by the Gulf of Guinea of the Atlantic Ocean on the south, Cote D'Ivoire on the west, Togo on the east, and Burkina Faso on the north. The capital of the country is Accra, and it also boasts of other important cities like Kumasi, Takoradi, and Tema, which are major trading centers. The population of the country is 23.8 million (Wellington et al 2011), with 32\% and 68\% living in the urban and rural areas respectively (Oppong-Anane, 2001). Ghana has a diverse agricultural and mineral resource based economy, and like many developing countries, agriculture accounts for about $54 \%$ of GDP and over $50 \%$ of foreign earnings, which makes Ghana's economy more dependent on agriculture (Owusu-Dabo et al 2009, Oppong-Anane, 2001). 
The agricultural sector is predominantly small scale and traditional, and it employs about 55\% of the workforce on a formal and informal basis (Owusu-Dabo et al 2009). The sector also provides over $90 \%$ of the food needs of the country (SRID 2001). The agricultural products of the country are mainly cash and food crops. Normally, similar cash crops are grown on the same farmland (monocropping) but different food crops are grown together in the same farmland (mixed-cropping). There are different types of food and cash crops in the country and these are grown in different ecological zones and regions of the country as the main agricultural activities of the farmers in those regions (Oppong-Anane, 2001). The cash crops mostly grown in the forest zone are tree crops like cocoa, oil palm, coffee and rubber while the food crops in this area are maize, plantain, cocoyam and cassava, which are normally grown together as mixed crops (Oppong-Anane, 2001). The predominant cash crops in the middle belt and the northern sector are tobacco and cotton while maize, legumes, cocoyam or yam are the main food crops in the middle belt (Oppong-Anane 2001). The main food crops in the northern sector are sorghum, maize, millet, cowpeas, groundnuts and yam.

Ghana cultivated 5,750 hectares of tobacco of the WHO region and was ranked $75^{\text {th }}$ in the production of tobacco leaves in the world in 2007 (Wellington et al 2011; ACS and WLF, 2009). The country also produced 2700 tons of tobacco and exported 2455 tons in the same year and tobacco export ranked $17^{\text {th }}$ of the total commodities exported by Ghana that year (Wellington et al 2011). Even though tobacco is considered one of the viable commercial crops providing revenue and employment for farmers in the regions where they are grown, it is one of the least contributors of foreign earnings for the country ${ }^{3}$. In spite of this, Ghana's tobacco control effort is often hindered by the Ministries of Agriculture and Finance, on grounds that adopting stricter tobacco control measures may cause farmers and other workers to lose their jobs and also have a negative impact on the country's GDP.

\footnotetext{
${ }^{3}$ The exact contribution of tobacco to the GDP and percentage of employment offered by the tobacco sector is lump up together and presented as the agricultural sector. The data so far only talks about the amount of export and total volume of tobacco produced unlike Malawi where specifics were given on tobacco.
} 
Ghana's experience with tobacco dates back to the end of World War II, when the veterans returning from the war introduced the product to the country. Since then, Ghana like many African countries, has witnessed the active operation of the tobacco industry for more than 50 years starting from 1948 (see Table 1.1). Ghana at different periods had tobacco manufacturing plants and leaf buying companies, including state-owned tobacco manufacturing and leaf buying companies (Owusu-Dabo et al 2009; Wellington et al 2011). The presence of these companies encouraged active tobacco leaf farming, which provided raw materials for the local cigarette manufacturing until 2006 when the last company left the country (Interview 2010). In spite of the long activities of the tobacco industry in the country, the prevalence of cigarette smoking is generally low in Ghana, accounting for $10.6 \%$ among adult males compared to $24 \%$ of the U.S and other global levels like $82 \%$ of Afghanistan, $67 \%$ of Ukraine, and $77 \%$ of Yemen and below $1 \%$ for females compared to $18.3 \%$ of U.S (Owusu-Dabo et al 2009; Wellington et al 2009; ACS and WLF 2009; Eriksen, Mackay, and Ross 2012).

The tobacco products consumed are the regular cigarettes, pipe smoking, sniffing, oral or nasal use of smokeless tobacco and locally, the chewing of dried tobacco leaf known as tawa mostly by the elderly (Wellington et al 2011; Interview 2010). The low prevalence of tobacco consumption can be attributed to cultural and religious reasons - traditional, Islam and Christianity-, which abhors smoking especially among women in Ghana as in many developing countries, (Cairney et al 2012). Culturally, tobacco smoking is considered a disrespectful and immoral act equivalent to prostitution especially among the Akans, the largest ethnic group in the country (Interview 2011). Traditional authorities, values, and practices of the ethnic group have strong impact on every day practices and behavior (Brenya and Asare 2011).The perception is not different for the other ethnic and religious groups because of the intermarriage of religious and cultural beliefs and practices and their impact on every day values and practices. 
Table 1.1 Timeline of Tobacco Production and Manufacturing, and Control Efforts (Including the Adoption of Voluntary Agreements) in Ghana

\begin{tabular}{|c|c|}
\hline \multicolumn{2}{|r|}{ Ghana } \\
\hline 1948 & $\begin{array}{l}\text { BAT partnered with local groups to establish a tobacco warehouse and began selling cigarettes imported } \\
\text { into the country }\end{array}$ \\
\hline 1951 & Gold Coast Tobacco Company established \\
\hline 1952 & $\begin{array}{l}\text { Pioneer Tobacco Company was established to champion domestic tobacco leaf cultivation and } \\
\text { manufacturing of cigarette in Ghana }\end{array}$ \\
\hline 1952 & Official manufacturing of cigarettes in Ghana \\
\hline 1957 & Ghana obtained Independence from the British \\
\hline 1959 & Pioneer Tobacco Company took over Gold Coast Tobacco Company \\
\hline 1962 & $\begin{array}{l}\text { Nkrumah's government passed law to take over tobacco marketing but the private companies came back } \\
\text { after the overthrow of his government in } 1966\end{array}$ \\
\hline 1971 & $\begin{array}{l}\text { The government established Ghana Tobacco Leaf Company to manage the marketing of tobacco } \\
\text { products }\end{array}$ \\
\hline 1976 & $\begin{array}{l}\text { Acheampong's government passed a law to take over ownership of private cigarette manufacturing and } \\
\text { tobacco leaf companies and organizations }\end{array}$ \\
\hline 1976 & $\begin{array}{l}\text { International Tobacco Ghana was establish to take over the marketing and production of all tobacco } \\
\text { products }\end{array}$ \\
\hline 1980 & $\begin{array}{l}\text { Government issued directive to prohibit smoking in government facilities, offices, and public places, } \\
\text { including restaurants and cinemas. }\end{array}$ \\
\hline 1982 & Ministry of Health directives to ban tobacco advertisements on TV, radio and the print media \\
\hline 1988 & $\begin{array}{l}\text { The Leaf Development Company was established in } 1988 \text { to produce tobacco leaf for the local market } \\
\text { and to lay the basis for a future export industry }\end{array}$ \\
\hline 1989 & International Tobacco Ghana was privatized \\
\hline 1993 & Ghana Committee on Tobacco Control was established \\
\hline 1999 & $\begin{array}{l}\text { BAT merged with Meridian Tobacco Company and became the sole local manufacturer of tobacco } \\
\text { products in Ghana }\end{array}$ \\
\hline 1999 & $\begin{array}{l}\text { Ghana signed the Lome, Togo Declaration on the Contribution of Parliamentarians to Tobacco Control } \\
\text { in the African Region }\end{array}$ \\
\hline 2000 & $\begin{array}{l}\text { Ghana joined GTSS and first national GYTS survey was conducted to determine prevalence of tobacco } \\
\text { among the youth }\end{array}$ \\
\hline 2001 & $\begin{array}{l}\text { The immediate Director General of Ghana Health Service complained about the painting of the biggest } \\
\text { market in Accra with BAT products, and this generated a national debate about tobacco control }\end{array}$ \\
\hline 2002 & Ghana became a member of Quit and Win International Smoking Cessation Program \\
\hline 2002 & Ghana National Tobacco Control Steering Committee inaugurated \\
\hline 2003 & First national tobacco control bill drafted for approval by Cabinet \\
\hline 2003 & Demographic and Health Survey conducted to assess tobacco prevalence \\
\hline 2004 & Ghana signed and ratified the FCTC \\
\hline 2005 & Second national GYTS survey conducted to determine tobacco prevalence among the youth \\
\hline 2005 & Tobacco Bill re-drafted to reflect FCTC provisions and sent to Cabinet for consideration \\
\hline 2006 & $\begin{array}{l}\text { Ghana chaired one of the committee meetings of the first session of the FCTC Conference of Parties held } \\
\text { in Geneva }\end{array}$ \\
\hline 2006 & Ministry of Health Directives to ban smoking in all Ministry of Health facilities \\
\hline 2006 & $\begin{array}{l}\text { Ministry of Transportation directive to ban smoking in public and private commercial transport including } \\
\text { the GPRTU and Inter City Buses, and on both domestic and international flights transport and buildings, } \\
\text { ports, and stadia }\end{array}$ \\
\hline 2006 & BAT closed down its manufacturing company and relocated to Nigeria \\
\hline 2007 & $\begin{array}{l}\text { Ghana chaired one of the committee meetings of the second session of the FCTC Conference of Parties } \\
\text { meeting held in Thailand }\end{array}$ \\
\hline 2007 & $\begin{array}{l}\text { Ministry of Health directives to compel all importers of tobacco products to register their products and } \\
\text { comply with the Food and Drugs Board regulatory requirements }\end{array}$ \\
\hline 2007 & $\begin{array}{l}\text { Ministry of Health, the Tobacco Control Steering Committee, and the Ghana Tourist Board reached } \\
\text { voluntary agreement with owners of entertainment industry to create smoke free area for nonsmokers }\end{array}$ \\
\hline
\end{tabular}




\begin{tabular}{|l|l|}
\hline 2007 & Institution of a five years plan of action on tobacco control \\
\hline 2008 & Ghana Demographic Health Survey conducted to determine national tobacco prevalence among the adult \\
\hline 2008 & $\begin{array}{l}\text { Ghana National Tobacco Control Steering Committee reached an agreement with importers to disclose } \\
\text { the content of tobacco products to be imported into the country }\end{array}$ \\
\hline 2009 & Another national GYTS survey conducted to determine tobacco prevalence among the youth \\
\hline 2009 & $\begin{array}{l}\text { Ministry of Education developed a School Health Education Policy that promote healthy lifestyle and } \\
\text { prohibit the use of tobacco by children }\end{array}$ \\
\hline 2009 & $\begin{array}{l}\text { Ghana Food and Drug Board directed all tobacco imported into Ghana to have the approved health } \\
\text { warnings and cover the required size }\end{array}$ \\
\hline 2010 & Ghana hosted the Second Meeting of the Working Group of the FCTC on Article (17) and (18) \\
\hline 2011 & $\begin{array}{l}\text { Attorney General's Department announces that a comprehensive Public Health Bill which has a tobacco } \\
\text { control provision has been sent to cabinet for approval instead of the draft Tobacco Bill. }\end{array}$ \\
\hline 2011 & $\begin{array}{l}\text { Ministry of Health directives mandating the posting of no smoking signs on the premises of all health } \\
\text { facilities }\end{array}$ \\
\hline 2011 & Parliament debate tobacco bill and send it to Select Committee on Health for Amendment \\
\hline
\end{tabular}

Source: (Owusu-Dabo et al 2009; Owusu-Dabo et al 2010; Wellington et al 2011; Wellington, 2007)

For instance, the Seventh Day Adventist Church educates its members against smoking through its temperance program (Cairney et al 2012; Interview 2011). The temperance program is the process whereby the church offers education on healthy choices for members. In spite of the low prevalence, Ghana has adopted certain tobacco control agreements to halt the anticipated health hazards associated with tobacco consumption.

\section{Malawi}

Malawi is landlocked independent republic, located in southern part of African, west of Mozambique and south of Tanzania. The country obtained its independence from the British in 1964. The capital of Malawi is Lilongwe, with other major cities as Zomba and Blantyre. Malawi has a total population of approximately 15.26 million (Eriksen, Mackay and Ross 2012). The country is one of the world's least-developed countries, with an economy based on subsistence agriculture and over 90 percent of its population rural. Tobacco growing is a major economic activity, accounting for a high percentage of Malawi's earnings, which makes it the country that heavily relies on tobacco followed by Zimbabwe. Tobacco is often referred to as green gold by Malawians, and it accounts 
for over $70 \%$ of total foreign earnings, $43 \%$ of agricultural gross domestic product, $13 \%$ of total gross domestic product, and 23\% of the tax base of Malawi (Otanez et al 2007; 2009; Otanez et al 2006; Interview 2010/2011). Otanez et al (2009: 1759) indicate that the tobacco sector directly employs between 600,000 to 2 million - $12 \%$ to $40 \%$ - members of the country's workforce of 5 million.

Malawi also has $2.95 \%$ of its total agricultural land allocated to tobacco farming (Eriksen, Mackay and Ross 2012). Moreover, it exports about 5\% of the world's tobacco and $2 \%$ of the world's total production (Semu-Banda 2007). Malawi has a high male smoking prevalence rate of about 22.8\% (Eriksen, Mackay, and Ross 2012) compared to 10.6\% of Ghana (Owusu-Dabo et al 2009). The smoking prevalence among the females is about $1.7 \%$. The low prevalence of smoking rates for women in relation to men is often attributed to the influence of the Christian and traditional religion as well as the cultural practices that abhor smoking among women (ACS and WLF, 2009; Interview 2011).

Tobacco production in Malawi dates back to the 1800s when settlers from the west introduced the growing of tobacco leaf to the country (See Table 1.2). Since then, tobacco leaf farming has become a major activity of the country and has attracted many leaf buying and selling companies who have become the face of the tobacco industry network in Malawi. The country produced 155, 000 hectares of tobacco in 2007 (ACS \& WLF, 2009). Tobacco activities (production and sales) in Malawi have been spearheaded by the international leaf buying companies and tobacco auction companies such as Alliance One International, Universal Corporation, and Limbe Leaf Tobacco, etc. 
Table 1.2: Timeline of Tobacco Production and Control Efforts (including the Adoption of Voluntary Agreements) in Malawi

\begin{tabular}{|c|c|}
\hline \multicolumn{2}{|r|}{ Malawi } \\
\hline 1889 & $\begin{array}{l}\text { Settlers from Virginia, United States introduce tobacco to Malawi and the country started } \\
\text { commercial production of tobacco }\end{array}$ \\
\hline 1893 & Malawi started exporting tobacco \\
\hline 1938 & Tobacco Control Commission was established to promote tobacco growing activities \\
\hline 1962 & $\begin{array}{l}\text { Limbe Leaf tobacco company (a subsidiary Universal Leaf Tobacco Company of the United } \\
\text { States of America) was incorporated and registered in Malawi }\end{array}$ \\
\hline 1964 & Malawi gained independence and became a republic \\
\hline 1970 & $\begin{array}{l}\text { International tobacco manufacturing companies identified Malawi as an ally for fighting tobacco } \\
\text { control }\end{array}$ \\
\hline 1972 & $\begin{array}{l}\text { Government enacted Special Crop Act that limited the production of tobacco to estate owners } \\
\text { with no exception for small landholders }\end{array}$ \\
\hline 1983 & $\begin{array}{l}\text { Nick Hauser representing the International Consortium of Tobacco companies meets with David } \\
\text { C.W. Kambauwa -Malawi's undersecretary of Agriculture - in Italy to develop a program to } \\
\text { work with Malawi to promote tobacco's economic importance }\end{array}$ \\
\hline 1985 & $\begin{array}{l}\text { A Philip Morris official gives a speech to the company's executives and notes that the company } \\
\text { lobbies Malawi to pressure the FAO to take a pro-tobacco stance in FAO publication on tobacco }\end{array}$ \\
\hline 1989 & $\begin{array}{l}\text { Labor and Human Rights Activists began to draw attention to the use of child labor in Malawian } \\
\text { tobacco production }\end{array}$ \\
\hline 1990 & Malawi liberalized tobacco farming to allow small landholders to produce tobacco again \\
\hline 1992 & Malawi reintroduced the allowance system that set quotas for tobacco production \\
\hline 1994 & $\begin{array}{l}\text { Constitutional Provision protecting the economic exploitation and interference of education of } \\
\text { children (which helps to protect child labor on tobacco farms) }\end{array}$ \\
\hline 1996 & The allowance system was abolished \\
\hline 1999 & $\begin{array}{l}\text { Smoking ban in some public and private places including Air Malawi domestic flights, airports } \\
\text { and in fuel stations was adopted }\end{array}$ \\
\hline 2000 & BAT and Philips Morris policy statement against child labor in tobacco production \\
\hline 2000 & $\begin{array}{l}\text { Tobacco Control Commission of Malawi argues that tobacco control would reduce Malawi's } \\
\text { tobacco earnings by } 10 \% \text { a year }\end{array}$ \\
\hline 2000 & $\begin{array}{l}\text { ITGA conducts media road show in Malawi to discredit World Bank evidence of public health } \\
\text { benefits of tobacco control }\end{array}$ \\
\hline 2000 & $\begin{array}{l}\text { ITGA works through Malawi task force on the FCTC to attempt to undermine and delay } \\
\text { meetings of FCTC working Group }\end{array}$ \\
\hline 2000 & $\begin{array}{l}\text { BAT and ITGA lobbies Malawi and other tobacco-growing countries to contribute to the FCTC } \\
\text { draft treaty text in which protocol language is weaker than the language of the original proposal }\end{array}$ \\
\hline 2000 & $\begin{array}{l}\text { Malawi signs Nairobi Declaration at the Inter-country Meeting on Tobacco Control Policy and } \\
\text { Programming }\end{array}$ \\
\hline 2001 & $\begin{array}{l}\text { Malawi signs Johannesburg Declaration at meeting of } 21 \text { countries from the WHO African } \\
\text { Region }\end{array}$ \\
\hline 2001 & $\begin{array}{l}\text { Malawi refuse to sign Algiers Declaration ratified at the consultative meeting of the WHO } \\
\text { African Region }\end{array}$ \\
\hline 2002 & $\begin{array}{l}\text { Malawi signs Abidjan Declaration at the consultative meeting of the WHO African Region on } \\
\text { FCTC }\end{array}$ \\
\hline 2002 & $\begin{array}{l}\text { Malawi signs Lilongwe Declaration at the } 4^{\text {th }} \text { sub-regional meeting of African countries on } \\
\text { FCTC }\end{array}$ \\
\hline 2003 & $\begin{array}{l}\text { FAO releases report on the impact of tobacco control and the FCTC on world economies that } \\
\text { notes Malawi's extreme reliance on tobacco }\end{array}$ \\
\hline
\end{tabular}




\begin{tabular}{|l|l|}
\hline 2003 & World Health Organization surveys tobacco prevalence in Malawi \\
\hline 2005 & WHOSIS Survey conducted \\
\hline 2005 & $\begin{array}{l}\text { Ministry of Health Directives to ban radio and television advertisement and also to require } \\
\text { advertisement in print to carry a health warning }\end{array}$ \\
\hline 2005 & Ban on tobacco sponsorship and promotional activities for sports and entertainment issued \\
\hline 2006 & GYTS surveys tobacco prevalence among the youth \\
\hline 2008 & Introduction of excise tax on imported cigarettes that does not use Malawian tobacco \\
\hline 2009 & Nyasa Tobacco Manufacturing Company established \\
\hline 2010 & $\begin{array}{l}\text { Adoption of a Tenancy Bill that makes it illegal to hire any child below the age of } 18 \text { years to } \\
\text { work on the tobacco farm }\end{array}$ \\
\hline
\end{tabular}

Source: (Makoka et al 2011; Otanez et al 2006, 2007, 2009; Semu-Banda 2007; Munthali 2009)

The tobacco growers associations such as the Tobacco Association of Malawi (TAMA) and Tobacco Tenants and Allied Workers Union of Malawi (TOTAWUM), and the Ministries of Agriculture and Finance also play active roles in the tobacco activities of Malawi (Makoka et al 2011; Otanez et al 2006; Otanez et al 2007; Semu-Banda 2007). The British American Tobacco Company, which is the major buyer of Malawian tobacco exported by the leaf companies, also plays a limited role in the country. The company has a subsidiary known as BAT- Malawi, which serves as a distributor of its cigarettes in the country (Interview 2010). The leaf buying companies and their affiliates are influential in the politics of tobacco control in the country and are often credited with hindering Malawi from signing and ratifying the FCTC international protocol as well as obstructing the adoption of legally enforced tobacco legislation in the country. In spite of the activities of the tobacco interest groups, certain voluntary agreements have been adopted to control the consumption of tobacco and its health hazards as shown in Table 2 above.

The general argument espoused by the tobacco control literature is that countries that heavily depend on tobacco or have a domestic tobacco manufacturing plant are less likely to adopt stricter tobacco control policies (Cairney, 2007). At the time of their independence, both Ghana and Malawi had substantial tobacco activities going on. Ghana was home to several tobacco manufacturing plants including state-owned tobacco manufacturing and leaf buying companies, which encouraged domestic tobacco farming (Owusu - Dabo et al 2009). Malawi also had a lot of tobacco leaf buying companies 
that made tobacco farming attractive in the country (Otanez et al 2006). However, the tobacco activities of Ghana have eventually dwindled due to tobacco control activities (Interview 2011). Ghana has made more advances with the tobacco control through the directives of the Ministry of Health and other government ministries and has signed and ratified the FCTC protocol. Malawi on the other hand, has not sign nor ratified the FCTC protocol ${ }^{4}$ and is more dependent on tobacco products. In addition, a domestic private tobacco manufacturing company has been established in Malawi recently (Otanez et al 2007, Makoka et al 2011).

Both Ghana and Malawi were former colonies of Britain and current members of the Commonwealth. The Commonwealth is known to be a venue where countries share significant knowledge on how to address common policies and problems, and was used as a venue to convince members to sign the FCTC protocol (Leichter 1983; Krishnan et al 2003; Commonwealth Secretariat 2007). Furthermore, both countries are members of the WHO and have some form of tobacco control activity going on, which includes significant evidence of contacts with actors from the developed countries. The purpose of the contact is to enable each of the countries to adopt domestic tobacco laws or voluntary agreements on tobacco control to prevent a tobacco epidemic. As the descriptive analysis above portrays, both countries have similar system of government and policy making processes. In addition, the behavior of the Parliament has also been similar, which has forced the directives of the Ministries of Health to be the main tobacco control instrument. In that regard, Ghana seems to have made more progress than Malawi. Therefore, this study seeks to understand the policy behavior of the two countries and explain the factors that influence policy adoption in each country. It analyzes the processes leading to the adoption of tobacco policies and voluntary agreements in Ghana and Malawi to explain the politics of tobacco control in each country.

\footnotetext{
${ }^{4}$ http://www.fctc.org/dmdocuments/ratification_latest_Bahamas.pdf
} 
The study adopts the most similar systems design, which helps to analyze the similarities in policy behavior and possible variations among the countries (Manheim et al 2006, Peters 1998). It examines the effects of two important factors, tobacco growing economy and a state-owned tobacco company on the adoption of restrictive tobacco control laws based on the history of tobacco of the two countries. The study also examines how signing and ratifying the FCTC impacts the adoption of tobacco control policies bearing in mind that Ghana was among the first forty countries that signed and ratified the FCTC at an early stage while Malawi has not yet done so. In addition, the study investigates the influence of tobacco companies on countries with different levels of corruption to ascertain if corruption could be a key factor to explain the policy behavior of the countries. The Transparency International Corruption Perception Index (2009) ranks Ghana as 69 and Malawi as $89^{5}$. In spite of the fact the countries are in different geographic regions of Africa - Ghana in Western and Malawi in Southern Africa ${ }^{6}$ - they both face similar tobacco-related problems because they are at the same stage of the tobacco epidemic (Lopez et al 1994, Cairney et al 2012; WHO 2008, Shafey, et al., 2009; also see Table 1.3 and 1.4).

Ghana and Malawi are both in the first stage of the tobacco epidemic where smoking among men is generally low. Also, fewer women than men smoke because women are culturally and religiously discouraged from smoking (Lopez et al 1994, Asma and Pederson 1999, Pampel 2008). In addition, there is no political will among the political leaders to control tobacco because tobacco related health hazards have not become a major public health problem. Lopez et al (1994) argue that most developed countries went through all the stages of the epidemic because the health hazards related to tobacco consumption took decades to become apparent. They maintain that the countries at the early stages of the epidemic can avoid tobacco hazards by adopting early intervention measures against

\footnotetext{
${ }^{5}$ The lower the rank, the greater the corruption

${ }^{6}$ The information on the profile of these countries can be obtained from the Foreign and Commonwealth Office website at http://www.fco.gov.uk/en/travel-and-living-abroad/travel-advice-by-country/. Retrieved 10/16/2009.
} 
Table 1.3: Prevalence and Stages of the Tobacco Epidemic among the Developing Countries

\begin{tabular}{|c|c|c|c|c|c|c|c|c|c|}
\hline \multirow{3}{*}{ Country } & \multirow{3}{*}{$\begin{array}{l}\text { Stage of } \\
\text { Tobacco } \\
\text { Epidemic }\end{array}$} & \multicolumn{8}{|c|}{ Percentage Adult Consumption Rate } \\
\hline & & \multicolumn{2}{|l|}{ Male } & \multicolumn{2}{|l|}{ Female } & \multicolumn{2}{|l|}{$\begin{array}{l}\text { Total } \\
\text { Men }\end{array}$} & \multicolumn{2}{|c|}{$\begin{array}{l}\text { Total } \\
\text { Female }\end{array}$} \\
\hline & & Cigarette & $\begin{array}{l}\text { Other } \\
\text { Tobacco } \\
\text { Products }\end{array}$ & Cigarette & $\begin{array}{l}\text { Other Tobacco } \\
\text { Products }\end{array}$ & TCCP & TA & TCCP & TA \\
\hline Ghana & 1 & 8.8 & 1.3 & 0.1 & 0.1 & 10.8 & 7.4 & 4.0 & 0.7 \\
\hline Malawi & 1 & 16.6 & 4.5 & 0.3 & 0.2 & 20.0 & 20.5 & 9.0 & 4.8 \\
\hline
\end{tabular}

TCCP =Tobacco Control Country Profile; TA= Tobacco Atlas 2009

Source: Pampel, Fred 2008, WHO 2008, a = Khan et al 2006; Flora et al 2009, b = White, Vicki, 2009, c = Youssef et al 2002

tobacco consumption. The adoption of relevant best practices of tobacco control from the developed countries can prevent the Ghana and Malawi from going through all the stages of the tobacco epidemic.

\section{The Role of International Actors in Tobacco Control}

It is generally believed that the tobacco control activities of government agencies, international governmental and nongovernmental organizations, and individuals can counter the activities of the tobacco industry. The collaboration between these tobacco control organizations provide good prospects for reversing the anticipated tobacco epidemic in the countries that are yet to adopt comprehensive tobacco control legislation such as Ghana and Malawi (Cairney et al 2012). Furthermore, the activities of knowledge transfer agents from the developed countries aimed at transferring tobacco control expertise to the developing countries will help to prevent the tobacco epidemic if Ghana and Malawi move soon enough to adopt and enforce the knowledge into comprehensive tobacco control laws.

\section{International Research Organizations and Government Agencies}

International research organizations and government agencies from the developed countries play roles considered crucial for the tobacco control in developing countries, including Ghana and 
Table 1.4:

\begin{tabular}{|ll} 
Stages & $\begin{array}{l}\text { Prevalence among male } \\
\text { and female }\end{array}$ \\
\hline Stage 1 & $\begin{array}{l}\text { Male prevalence less than } \\
15 \% \text { and female less than } \\
10 \% \text { due to social and } \\
\text { cultural discouragement }\end{array}$ \\
Stage 2 & $\begin{array}{l}\text { Male prevalence continues to } \\
\text { rise and peaks at 50-80\%. } \\
\text { That of women continues to } \\
\text { rise but lags behind that of } \\
\text { men by one to two decade. } \\
\text { Similar Prevalence for } \\
\text { different socio-economic } \\
\text { classes but is slightly higher } \\
\text { with upper class }\end{array}$
\end{tabular}

Stage 3 Male prevalence exceeds $60 \%$ for a long time and begins to drop to about $40 \%$

by the end of the phase. Smoking is usually lower among the middle-aged and older men, most of whom are ex-smokers and female prevalence peaks around 35 $45 \%$ and begins to decline.

Stage 4

Smoking prevalence continues to decline among both male and female smokers in comparable manner. Female prevalence decrease around $10-15 \%$ and is typically around $30 \%$ and that of male is around 33$35 \%$.

\section{Characteristics of the Stages of the Tobacco Epidemics}

\section{Per Capita Cigarette \\ Tobacco Related Health} smoking

Generally low, less than 500

cigarettes and mostly smoked by males

Per capita consumption varies between 1000 and 3000 per annum and men still consume the highest. Annual consumption among men is probably between 2000 - 4000 hazards and Deaths

\section{Beginning of the smoking} epidemics. Very few male deaths by the end of the phase. Rare Lung cancer comparable to those in non-smokers

Tobacco use is causing about $10 \%$ deaths among males and is comparatively low among women. Male lung cancer has risen 10 times from bout $5 / 100000$ to $50 / 100000$. The rate among women is still small about $8-10 / 100000$

Male consumption is about 3000 - 4000 cigarettes per annum and women consume between 1000 - 2000 cigarettes per year towards the lower end of this stage. The difference between male and female smoking is about 5\% and smoking among women is related to their age.

Per capita consumption of tobacco products is decreasing among both sexes partly because of the knowledge on the health hazards of tobacco consumption. Smoking is lowest among the educated who have responded to the negative health effect

There is rise in male smoking related deaths to about $25-30 \%$ from $10 \%$ within three decades. Deaths among the middle-aged (35-65 yrs) are about one in three deaths and females deaths is about $5 \%$ of all deaths. Male lung cancer rate is about 110 -

$120 / 100000$ and tobacco related diseases death among women is about $25-30 / 100000$.

Male tobacco related deaths peaks at $30-35 \%$ of all deaths and is $40-45 \%$ among the middle-aged. Female deaths will be still rising around 20$25 \%$ of all deaths. Lung cancer rates among men falls to about $20 \%$ after the peak. Mortality for both sexes decline

\section{Nature of Tobacco} Control Policies

Generally no organized and effective tobacco control policies

Tobacco control activities not well developed. Information and education on the hazards of tobacco are sporadic and not well organized

afterwards.

Strict tobacco control laws on smoke free public places and transport but not for smoke free workplace. There are organized tobacco contro programs often taught in schools
There are effective tobacco control measures. Smoke -free environmental laws and workplace laws are common

\section{Political Will}

Lack of political support to control tobacco because health hazards have not become apparent

Lack of public and political support likely to undermine successful implementation of control laws because the hazards are not properly understood

Conditions for tobacco control measures are more favorable and the media supports laws on tobacco control. Society discourage smoking and smoking behavior is generally considered abnormal behavior

High public support for tobacco control policies and the public demand policies for smokers who wish to quit smoking but are finding it difficult to quit. Society continues to ridicule smoking habits. 
Malawi. Therefore, the study analyzes the activities of governmental agencies such as the International Development Research Center (IDRC), and the Research for International Tobacco Control (RITC) of Canada, the National Institute for Medical Research (NIMR) of the British Medical Research Council (BMRC), the Centers for Disease Control and Prevention (CDC) of United States, and the Global Youth Tobacco Survey (GYTS).These governmental agencies conduct research to control various diseases and also offer assistance for controlling diseases in other countries. For instance, the US Centers for Disease Control and Prevention undertakes extensive research aimed at disease control and prevention both in the US and abroad because of the interrelationships in global health.

The Office on Smoking and Health of the National Centers for Chronic Disease Prevention and Health Promotion under the Centers for Disease Control \& Prevention is a WHO collaborating center for global tobacco surveillance. The Center establishes a global tobacco surveillance system for tobacco control and also facilitates dissemination of surveillance data as well as advances and disseminates science and research for effective tobacco control programs. Additionally, the CDC through the GYTS also offers technical assistance to the WHO for global tobacco control (CDC 2009). The GYTS conducts surveys to identify the prevalence of tobacco among the youth in the developing countries as a way of helping the countries to adopt the right strategies to control tobacco consumption. The CDC provides financial and technical assistance for the Global Youth Tobacco Survey, including survey design and sample selection, training of research coordinators for fieldwork implementation procedures, data management and processing, initial tabulation of the data, and training for analysis to support enhanced surveillance systems in countries across all WHO regions. The GYTS assesses attitudes, perceptions and behaviors regarding tobacco use among youth and also publishes their data in 
peer reviewed journals to influence the thinking on the tobacco issue among scholars and policymakers (WHO 2005).

The Canadian government though the IDRC conducts research to assist the development agendas of other countries. The IDRC and the RITC engages in activities to ensure global adoption of tobacco control policies to prevent related health issues. The National Institute for Medical Research (NIMR) is a British research institute dedicated to conducting research on the life processes that are relevant to all aspects of health. The Institute is funded by the Medical Research Council (MRC), a publicly funded national organization that promotes research into all areas of medical and related science to improve the public health in Britain and abroad. The United States, United Kingdom, and Canada were selected because of their progress with tobacco control, which has resulted in a decrease in cigarette smoking (Warner 2000, CDC 1999, WCTOH 2000).

Even though the US has not ratified the FCTC, various states such as California have adopted comprehensive tobacco control laws (Asare 2007). Also, the NGOs based in these countries play a leading role in preventing global epidemics caused by diseases, among which tobacco related diseases loom large. The countries and their NGOs offer substantial amounts of aid and grants to the developing countries for tobacco control. Moreover, Marmor and Lieberman (2004) classify U.S, Canada, United Kingdom, and Australia as the English-speaking group with strong tobacco control outcomes. Studlar (2002:18) maintains that United States and Canada have similar histories of the impact of tobacco and that there has been convergence in the policies to control tobacco in the two countries. There is also collaboration between some UK based NGOs with organizations from the US and Canada to control tobacco consumption globally. For instance, the Bloomberg Initiative and Foundation offers grants to the International 
Union against Tuberculosis and Lung Disease based in Edinburgh, UK to disburse to tobacco control organizations and agencies in the developing countries (Interview 2010) ${ }^{7}$. The American Cancer Society also collaborates with the IDRC and UK Cancer Union to promote tobacco control in developing countries. In other words, there are lots of collaborations among these organizations to transfer tobacco control knowledge to the developing countries ${ }^{8}$.

Lopez et al (1994: 245) argue that the US and Canada are at the end of the third stage of the tobacco epidemics while the UK is already in the fourth stage (refer to Figure 1.3 and Table 1.4 above). They maintain that all the three countries went through the early stages of the epidemic before detecting the serious health consequences of the tobacco consumption. They further argue that in spite of the fact that the countries continue to experience massive tobacco related deaths and health problems, the U.S, UK, and Canada have been able to avoid the worst scenario projected to occur at the last stages of the epidemic because of the adoption of relevant tobacco control measures. The preliminary review of literature shows that the tobacco control agents from these countries are directly or indirectly sharing their experience with Ghana and Malawi to prevent the tobacco epidemic.

\section{International Governmental Organizations}

The transnational activities of the tobacco companies and the global nature of the tobacco epidemic make the role of international governmental organizations vital to the tobacco control of Ghana and Malawi. International organization such as the WHO promotes issues of public health globally. The WHO is also the umbrella body hosting the FCTC, and through the Tobacco Free Initiative, administers the implementation of the FCTC measures. The activities of the

\footnotetext{
${ }^{7}$ For more on this see http://www.tobaccocontrolgrants.org/

${ }^{8}$ Detail analysis of the collaboration between these organization is done in chapter three
} 
WHO are carried out by its six regional offices across the globe (Interview 2011). For instance, the tobacco control activities of the WHO in Africa are spearheaded by the WHO regional office for Africa, which has its headquarters in Brazzaville, Republic of Congo. The regional office conducts training, workshops, and conferences for member countries to ensure the adoption of tobacco control measures. It also engages in awareness and education programs and uses the Annual World No Tobacco Day celebrations to highlight the dangers associated with smoking. The WHO works closely with other governmental agencies and nongovernmental organizations to influence countries to sign and ratify as well as adopt relevant domestic tobacco control policies.

The Commonwealth of former British colonies is another intergovernmental organization that plays important advocacy role on tobacco control for its members. Krishnan et al (2003) note that Commonwealth member countries are distributed over five of the WHO regions, and that the Commonwealth is made up of 54 member countries with about 1.7 billion citizens who account for more than $25 \%$ of the population of the world. In addition, the Commonwealth played a crucial role in influencing member countries to sign and ratify the FCTC protocol in 2003 by commissioning research which was used to advise the Health Ministers of member countries on the importance of the FCTC protocol (Krishnan et al 2003, Latif 2004, The Commonwealth Secretariat 2007). The Conferences of the Commonwealth Health Ministers often serves as a lobby ground for some tobacco control organizations and NGOs such as the IDRC.

The IDRC often attends Commonwealth meetings to lobby for the adoption of comprehensive tobacco control policies by member countries to prevent the tobacco epidemic (IDRC 2004). Moreover, the British based tobacco company, British American Tobacco (BAT), 
is the second largest transnational tobacco company, with subsidiaries in about 65 countries including the two countries under study (Krishnan et al 2003). The members of the Commonwealth are made up of both developed and developing countries, some with best practices for controlling tobacco and others without, which provide incentives for learning from each other. Moreover, the Commonwealth countries have a history of cooperating with and learning from one another on policy issues of common interest (Leichter 1983; Collier and Messick, 1975). Therefore, the Commonwealth could be used to influence the transnational activities of the company and vice versa. Another organization of interest is the Framework Convention Alliance (FCA). The Framework Convention Alliance organizes the meetings of the Conference of Parties (COP) of the FCTC to deliberate and share new knowledge on the best practices for tobacco control and so the analysis of their activities is vital (FCA, 2001).

\section{Nongovernmental Organizations}

The study also examines the tobacco control activities of some nongovernmental organizations such as the Bill and Melinda Gates Foundation, the Bloomberg Global Initiative and Foundation, the American Cancer Society, the Physicians for a Smoke Free Canada, and Cancer Research UK (UKCR). It also investigates the activities of the International Union against Cancer (IUAC), the Campaign for Tobacco Free Kids (CTFK), and the International Union against Tuberculosis and Lung Disease (IUTLD). The NGOs help policy makers in developing countries to acquire the requisite skills for enacting laws and creating awareness and education on activities that generate global health problems through the provision of funds to domestic tobacco control actors. 
For instance, the Bill and Melinda Gates Foundation and the Bloomberg Foundation are US based NGOs that have been offering financial grants for research on cancer and AIDS to curb the global epidemic generated by these diseases, especially in developing countries. They also offer financial assistance for education and training on HIV/AIDS prevention as well as tobacco control to prevent its related diseases. The American Cancer Society also offers grants to tobacco control activists in developing countries to assist with activities aimed at pressuring their countries to adopt relevant tobacco control laws. The Society is currently administering the Africa Tobacco Control Consortium, which spearheads activities to promote the adoption of tobacco control laws in the region (Interview 2010). In much the same way, Physicians for Smoke Free Canada is a Canadian nongovernmental organization that has been undertaking activities to prevent the health hazards of tobacco in Canada and many developing countries.

The Physicians for Smoke Free Canada has been involved in helping countries in the southern part of Africa to adopt appropriate tobacco control policies (PHASA 2007). In addition, the UK Cancer Research collaborates with organizations like the American Cancer Society to ensure the adoption of effective tobacco control laws to prevent health hazards such as cancer. Other international nongovernmental organizations such as the International Union against Cancer (IUAC), the Campaign for Tobacco Free Kids (CTFK), and the International Union against Tuberculosis and Lung Disease (IUTLD) are actively involved in the global control of the consumption of tobacco products. The Campaign for Tobacco Free Kids, the American Cancer Society, and the IUTLD are the main organizations that disburse the funds from the Bloomberg Foundation for tobacco control (Interview 2010, 2011). In addition, the World Conference on Tobacco or Health (WCTOH) and the International Conference on Illicit tobacco 
Trade (ICITT) provide the venue for sharing of expertise on tobacco control through their periodic conferences 9 .

\section{Tobacco Control Literature}

The issue of tobacco and its hazards has obtained great prominence in scholarly writings in recent decades, even though tobacco use and its control is an old phenomenon (Borio 2001, Mamudu 2005). Generally, the studies on tobacco have been analyzed through the lenses of the effects of tobacco consumption on public health, the social and economic impact of tobacco, and/or the processes of tobacco control. Scholars focusing on public health examine the history of the evidence linking tobacco to health hazards. Some attribute the initial evidence of the health hazard of cigarette smoking to the1930s when the first medical proof of both the addictive nature of tobacco and the cancer hazard from smoking was detected by medical experts in Nazi Germany (Proctor 1999). Others trace the scientific discovery that links cigarette smoking and lung cancer to scientific reports of the British Medical Research Council (BMRC) published in 1957 on research conducted in United States and the United Kingdom (Collin et al. 2002;

Farquharson 2002; Proctor 1999, Leichter 1983).

Notwithstanding these claims, Asare (2007:10) and Mamudu (2005:7) argue that what energized and politicized the institution of a global tobacco regime were the reports of the Royal College of Physicians (RCP) of 1962 in the United Kingdom and that of the Surgeon-General of United States in 1964, which outlined the health hazards of cigarette smoking. Mamudu (2005:8) and the World Conference on Tobacco or Health (WCTOH, 1967) argue that the reports shifted the debate on tobacco from the analysis of its health effects to control and prevention. Asare

\footnotetext{
${ }^{9}$ The details on the specific activities of the IGOs, NGOs and epistemic communities will be reviewed in Chapter Four and Five.
} 
(2007) maintains that the discovery of the negative impact of second hand smoking in the 1980s also gave impetus to the emergence of the tobacco control regime. Bayer and Colgrove (2004) share a similar view, arguing that policymakers in the US were motivated by the desire to protect secondhand smokers from the harmful effects of tobacco. This is because passive smoking has serious negative effects on the health of people, especially children (Beyer and Brigden 2003). These studies present ideas that the focus on tobacco has clearly shifted and the recent emphasis is more on preventing hazards associated with tobacco consumption. A major part of the prevention measures is the promotion of policies that will curtail the activities of the tobacco industry, especially in the developing countries.

In attempt to provide adequate justification for the adoption of prevention measures, other scholars, especially those from the public health background undertake studies that analyze health hazards and deaths related tobacco (Taylor 1985, Glantz et al 1996, Jha \& Chaloupka, 2000, WB 1999, WHO 2008). Some of these scholars have attributed the largest share of premature and preventable deaths to cigarette smoking (Glantz et al 1996; Jha and Chaloupka, 2000; Taylor, 1985, Lando et al 2005). For instance, Beyer and Brigden (2003: ix) contend that a tobacco related death occurs worldwide every 10 seconds. Tobacco is also projected to account for the largest number of deaths globally by 2050, registering about 10 million deaths per annum (WHO-TFI 2007). Yet others scholars have examined the consequences and the trends of cigarette smoking globally and concluded that tobacco consumption and cigarette smoking is a great threat to future premature deaths in all countries - poor, rich, developed, or underdeveloped (Jha and Chaloupka, 2000, World Bank, 1999; WHO, 1997). For instance, the Research for International Tobacco Control (RITC) argues that the diseases related to tobacco have cumulative mortality rates that exceed the combined problems of AIDS, tuberculosis and 
complications of child births and serve as the most vital preventable health problem in recent times ${ }^{10}$. The findings and conclusions from these studies no doubt give impetus to the demand for the adoption of more tobacco control policies to prevent the tobacco epidemic, especially in the developing countries where there is rapid increase of tobacco activities in recent times.

In addition, studies that have analyzed the anticipated social and economic impact of adopting tobacco control measures as domestic policies have concluded that countries need to be proactive with tobacco control because of its effect on their economies and citizens (Licari and Meier 1997, Spill et al 2001, Feldman 2004, Manfredi and Maioni 2004, Ballard 2004). The studies indicate that tobacco control has important benefits for countries because of the negative effects of the tobacco industry, especially for developing countries. For instance, The WHOTobacco Free Initiative (2007) argues that the problem of tobacco for developing countries affect both health and development.

The report argues that tobacco not only drains the developing countries of their meager resources for sustainable development through the treatment of health hazards, but the related diseases render many members of the active workforce ineffective while killing a significant proportion of the productive labor force. The provision of such hard facts helps to support the promotion of the adoption of tobacco control policies, which are being projected as the only remedy for avoiding the anticipated epidemic. In that regard, the World Conference on Tobacco or Health (1994) claims the problems associated with the use of tobacco products such as cigarette smoking can be curtailed through adoption of effective regulatory policies and control measures to reduce per capita tobacco consumption. It argues that the adoption of such measures can reduce the menace posed by tobacco consumption.

\footnotetext{
${ }^{10}$ See (RITC: R T C) 1. G2 .C report retrieved from Website: www.idrc.ca/uploads/user-S/10547490910case4.pdf on $10 / 17 / 2009$
} 
In a similar way, Lopez et al (1994) have suggested that the adoption of effective control policies will prevent countries at the early stages of the tobacco epidemic from experiencing all the other stages. They further argue that Singapore was able to prevent tobacco related deaths because it adopted early intervention measures at Stage Two of the tobacco epidemic in the 1970s. The World Conference on Tobacco or Health (1994) also argues that the adoption of regulatory and effective tobacco control policies have resulted in a decrease in the per capita consumption of cigarettes by $10 \%$ in developed countries since the 1970 s. In the US, the total consumption of cigarettes has decreased from 633 billion cigarettes in 1981 to 479 billion in 1997 (Warner 2000:418).

Warner (2000) also argues that the antismoking campaign in the US has avoided close to 3 million tobacco related premature deaths in the first two decades up to the year 2000 . However, the reduction of tobacco consumption has been witnessed in the developed countries due to the adoption of tobacco control measures. The adoption of such measures in the developed countries has resulted in the expansion of tobacco activities in the developing countries as the tobacco companies have shifted their activities to those countries. Globally, the total volume of cigarettes exported to the developing countries increased from 2,017 to 2,172 billion units from 2004 to 2008 while the figures in the developed countries decreased from 1,297 to 1,170 billion units within the same period (Cairney et al 2012; Camilleri \& Morgan Stanley 2009).

In effort to curtail tobacco control activities globally, many studies have been conducted to examine the processes of tobacco control policy adoption in some countries. The studies range from those that examine the role of policy entrepreneurs and epistemic communities in pushing for the adoption of general control measures at the global level (Roemer and Taylor 2005) to those that focus on the reasons for the emergence of the WHO-FCTC as a global 
tobacco control regime (Mamudu, 2005). Additionally, others studies focus on the negotiations of the WHO-FCTC and its control measures (Framework Convention Alliance (FCA), 2001, World Bank 1999), while some have also examine the role played by interest groups to promote the adoption of tobacco control in individual countries (Malan and Leaver 2003; Asare 2009; Albaek 2004; Read 1996; Mamudu et al 2008; IDRC 1995; Yach and Bettcher 2000; Frey 1997; Sklair 2002; RITC 2006, Mamudu 2005). Other studies have also analyze the processes of tobacco control policy adoption through the lens of political institutions (Asare 2007; Kagan and Vogel 1993; Marmor and Lieberman 2004; Pross and Stewart 1994; Frankenberg 2004; Derthick 2002; Glantz and Balbach 2002; Givel and Glantz 2000; Fakhafakh et al 2002; Jha and Chaloupka 1999; Studlar 2002, 2003, 2004a, 2005, 2007b) and lesson drawing/diffusion (Studlar 1999, 2004a, 2004b, 2009; Asare 2007; Asare and Studlar 2009; Lopez et al 1994). The findings of such studies not only provide evidence of tobacco control to motivate other countries to emulate, but also provide countries who may be concern with preventing tobacco hazards with tools of how to address their problems based on what others have done.

Asare (2007:23) maintains that most of these studies emanate from and focus on tobacco control in the industrialized countries and the findings of the studies have generally concluded that almost all the industrialized countries who have adopted such measures have succeeded in their attempt at controlling tobacco, albeit to different degrees. While some of these studies concentrate on tobacco control policies of a single country (Pross and Stewart 1994; Read 1996; Sato 1999; Fakhfakh et al. 2002; Shimkhada et al. 2003; Ballard 2004; Feldman 2004; Manfredi and Maioni 2004; Licari and Meier 1997; Spill et al 2001), others focus on a comparative study of two or more countries (Asare 2007; Studlar 1999, 2002, 2004a, 2004b, 2005, 2007b; Jha and Chaloupka 1999; Kagan and Vogel 1995). Unfortunately, none of the studies examine the 
process of tobacco control in developing countries, despite evidence suggesting that the tobacco companies are directing their activities to the developing countries, which makes this study important to fill the gap in the literature.

Moreover, the studies that have reported that the tobacco companies are sending in substantial quantities of their products to developing countries following the success of the industrialized countries in controlling the consumption of tobacco identify Africa and Asia as the main target of the tobacco industry (Frey 1997; Jha and Chaloupka 2000; Sklair, 2002; Yach and Bettcher 2000). Additionally, there are reports confirming a rapid rise in tobacco consumption and anticipated health hazards in these continents due to the activities of the tobacco companies (World Bank 1999; WHO 1997, 2008). Therefore, a study that provides academic assessment of the politics and processes of tobacco control in certain countries in Africa as this one does will greatly aid in presenting a clear picture of the effort to address the tobacco epidemic and the reaction of the tobacco industry network to the adoption of such measures. The understanding of the nature of tobacco control activities in the developing countries may aid the global tobacco control effort given that the activities of the tobacco industry is general similar in many ways.

There is clear evidence that the tobacco companies adopt similar tactics such as using deceptive advertising to attract new users and employing various strategies to prevent the adoption of restrictive tobacco control measures in all the countries in which they operate (WHO 2004, 2008; World Bank 1999). For instance, the International Development Research Center (1995) argues that the tobacco companies resort to all manner of bad trading practices such as using attractive and vigorous deceptive advertising that presents high smoking habits in the developed countries to attract new smokers in the developing countries. In addition, there are 
evidence of the used of pictures of famous celebrities in tobacco advertisement as means of luring people to smoke.

The seriousness of the actions of the tobacco companies is that many people in the developing countries hold the people of developed countries in high esteem and emulate their practices and habits. Therefore, such deceptive advertisements are very effective in luring people, especially the youth, to cultivate the habit of smoking, which leads to the tobacco related health hazards. The World Health Organization (2004) maintains that some of the strategies used by the tobacco companies to undermine tobacco control in developing countries include infiltrating parliament to lobby policy makers and government officials as well as maintaining strong contacts with influential figures to avoid the adoption of strong tobacco control laws that will hamper their activities. The International Development Research Center (1995) indicates that in 1995, the Miss Ghana Contest was sponsored by a tobacco company known as "Embassy" and the Minister of Education presented the award.

Mamudu et al (2008:1692) argue that the tobacco companies and their affiliates hire public relations firms and sponsor studies on the economic impact of tobacco to convince governments that tobacco has positive benefits for their economies. They maintain that the tobacco industry have been trying to discredit the World Bank's (1999) landmark study which concluded that tobacco control generates unprecedented health benefits and does not harm the economies of countries. Mamudu et al (2008) further argue that the British American Tobacco has established a lobbying program targeting the World Bank and the International Monetary Fund in Washington, DC, because of the growing support of these financial institutions for tobacco control lending and policy on trade. 
Asare (2007:14) also notes that some tobacco companies pretend to care about the hazards of tobacco by carrying advertisements on television and discussing on their websites the health hazards of tobacco for both smokers and nonsmokers. He cites Philip Morris as one company that adopts such a strategy even though the company still explores new trading avenues to sell cigarettes. There is also evidence that the tobacco companies use their supporters to sabotage anti-smoking activities by infiltrating tobacco control organizations. The recent withdrawal of funding for tobacco control offered to the Canadian International Development and Research Center (IDRC) by the Bill and Melinda Gates Foundation attest to such strategy.

The Bill and Melinda Gates Foundation withdrew its funding because of the links of the Chair of IDRC board of governors - Barbara J. McDougall - to a subsidiary of the British American Tobacco (Wilson, 2010). The Foundation and tobacco control activists in Africa complained that the chair was not promoting comprehensive tobacco control policies because of her link to the tobacco companies. The World Health Organization (2004) argues that Philip Morris, in its 1981 report, indicates its commitment to counter the adverse effects of antismoking and tobacco control activities. The paper further presents a report by the company on a 5-year plan to study the tobacco control situation in Egypt in order to institute a counter action through increased public relations. The FCTC control measures have also been described as "developed world obsession being foisted on the developing world" by the tobacco companies (Hammond and Assunta, 2003).

The World Health Organization (2004) and Mamudu et al (2008) report that the tobacco companies manipulate the media and advertising companies and also sponsor people to speak in their favor at conferences to promote the activities of the tobacco industry network. The companies also conduct regular surveys and research to present findings favorable to their 
interests. In addition, warnings on the cigarette packs are written in languages that most illiterates in developing countries cannot read or comprehend. Barraclough and Morrow (2008), and Soerojo and Budiantoro (2007) argue that the tobacco companies use the cloak of corporate social responsibility to build good relations with government officials and the public to protect their economic activities. For instance, Soerojo and Budiantoro (2007:7) argue that the tobacco companies offer educational assistance and emergency response to national disasters for the poor as well as undertake environmental programs in Indonesia.

Soerojo and Budiantoro (2007) also state that the companies sponsor key national events such as the Independence Day Celebrations and major religious events, including the celebrations of the ascension and birth of Prophet Mohammed in Indonesia. They argue that the tobacco industry network sponsors major sporting and cultural events at both the local and national levels, while at the same time engaging in active advertising of their products, using all available channels such as billboards, televisions, posters, banners, and lampposts. They argue that the network members mostly target the youth and even use some of their sponsored events to give free samples of their products to the youth to try. In addition, many of their advertisements are placed at places where youth congregates, including sporting centers, schools, and malls. Barraclough and Morrow (2008) maintain that such strategy "overshadows" criticism and helps the companies to continue their operations because the policy makers find it difficult to adopt restrictive tobacco control policies that will hamper the operations of the tobacco industry network. My interviews reveal that the pattern is similar in both Malawi and Ghana. Therefore, these actions threaten the environment and the sustainable development of the developing countries. Moreover, presenting the facts in an academic study may generate new ideas on tobacco control for those countries that may help to address the tobacco issue. 
The World Conference on Tobacco or Health (1994) argues that there is a correlation between the adoption of restrictive tobacco control laws and the reduction in per capita cigarette smoking. For instance, Asare (2009) found that South Africa increased it tax revenue and decreased the level of tobacco consumption by adopting effective tobacco tax instruments. The World Conference on Tobacco or Health (1994) further argues that the increase in per capita smoking rate in developing countries is due to either the presence of weak tobacco control laws or their total absence. The World Bank (1999) argues that the developing countries can curtail the tobacco epidemic by adopting relevant tobacco control policies. It argues that the prevention of deaths in developing countries will depend on the type of the tobacco control policies they adopt and calls for the adoption of austere regulatory policies (Refer to Figure 1.3). The World Health Organization (2008) also maintains that developing countries stand to benefit more from adopting and enforcing effective tobacco control laws because the money used to treat tobaccorelated health hazards can be used for other developmental projects. Therefore, an overview of the actual nature of the tobacco control activities will be essential to the adoption of the requisite measures needed to address the problem in these countries.

The importance of a study of this nature stems from the fact that the tobacco companies are constantly adopting new strategies to protect their interests. With the plethora of research providing a clear evidence of the harm of tobacco, the companies have resorted to new strategies, some of which are enumerated above. The recent strategy adopted replaces the old tactics whereby the companies tried to reject the findings that ties tobacco consumption with health hazards as unscientific. The World Health Organization (2008) maintains that the tobacco companies have more resources and are more politically powerful than the tobacco control advocates who seek to protect children and non-smokers from tobacco or to help tobacco users to 
quit. Therefore the countries and policymakers need to do more to curtail the tobacco pandemic. The World Health Organization further argues that political commitment is needed for the adoption and enforcement of comprehensive domestic tobacco control policies because of the politics that normally characterize the adoption process. To Asare (2007) and Saloojee (1993), the importance of tobacco as a major economic commodity for many developing countries makes political commitment of policy makers more crucial. For instance, tobacco accounts for a large percentage of the gross domestic product of countries like Malawi and Zimbabwe (Jacobs et al 2000). Therefore, a ban of tobacco sales and growing for countries like Malawi could have a significant impact similar to a ban of oil sales for countries like Saudi Arabia, which makes political commitment very vital (Eldring et al 2000).

The developing countries have witnessed an increase in the production of tobacco products from the 1960s, which has contributed to the global expansion in tobacco production (Cairney et al 2012; Chapman \& Fahey 1989; FAO 2003; WHO 2004). The increased in the share of the global tobacco production of the developing countries was due to increases in tobacco production from countries such as Bangladesh, India, China, Brazil, South Korea, and Thailand. Generally, the tobacco production from the developing countries increased from 53\% in $1962-1964$ to $69 \%$ in 1985-1987 (WHO 1991). From 1980 to 2001, global production of tobacco leaf increased at an average $1.02 \%$, from 5.26 to 6.97 million tons (Jaffee 2003). Within the same period, the tobacco production in the developed world - including the US, EU and Japan - declined by $2 \%$ and $3.1 \%$ per annum in the 1980 s and 1990 s respectively, decreasing from 1.99 million tons in 1980 to 1.29 million tons in 2001 (Jaffee, 2003).

However, tobacco production in developing countries increased from 3.26 million tons in the 1980 s to 5.68 million tons in 2001, increasing the share of developing countries' global 
tobacco production from $62 \%$ to $81 \%$ over the period (Jaffee 2003). The production of tobacco in the developing countries grew by $3.8 \%$ in the 1980 s but saw no significant growth in the 1990s (Jaffee 2003). In recent times, the increase in the share of the developing countries to the global tobacco production has also been influenced by the increases in the production by countries such as Malawi and Zimbabwe (until the last 5 years) (Jaffee 2003). Malawi increased its tobacco production from 124,500 tons in 1990 to 138,200 in $2002^{11}$ and seven of eleven countries that produced $80 \%$ of the world's tobacco leaf in 2003 were from the countries in the developing regions (FAO 2003; World Bank 2003; Cairney et al 2012).

The contribution of tobacco to tobacco economies in the developing countries makes it difficult for such countries to adopt stricter tobacco control policies because of the impression that tobacco products are essential to their balance of payments (RITC 2006; Schreiner and Dalesio 2008; WHO 2004). It is the failure to adopt comprehensive tobacco policies that has encouraged the tobacco companies to take advantage of the weak laws to send substantial quantities of their tobacco products to such countries, mostly in Africa (Frey 2000; Jha and Chaloupka 2000; Sklair 2002; Yach and Bettcher 2000). Therefore an academic study on the nature of tobacco control activities may help the policymakers to develop the commitment to adopt the requisite measures that may help the countries to address the tobacco issue. Asare (2009:105) argues that the commitment of the government of the African National Congress of South Africa enabled the country to adopt comprehensive tobacco control policies that has made it a leader in tobacco control in sub Saharan Africa. The example of South Africa offers a measure of hope to developing countries, especially in Africa who may want to travel that path to emulate.

\footnotetext{
${ }^{11}$ This excludes about 5000 to 10000 tons smuggled to neighboring countries and imported back to Malawi
} 
Waverley (2007) supports the assertion that tobacco control will generate more revenue for developing countries, arguing that the South African government increased its revenue with an increase in tobacco excise taxes. To the World Health Organization (2008), every \$1 used for tobacco control in low and middle income countries can generate revenue of about $\$ 5000$. It argues that even though most governments generate over US\$200 billion in tobacco revenues, they spend less than $1 / 5$ of $1 \%$ of that amount on tobacco control. This is partly because the tobacco producing companies use deceptive tactics to maintain their hold on the developing countries to prevent them from adopting effective tobacco control measures. The report identifies one of the deceptive tactics used on the developing countries by the tobacco companies as portraying the notion that tobacco production creates jobs in a context of a high unemployment rate. However, the International Development Research Center of Canada refutes the job creation claim of the tobacco companies, arguing that the transnational companies are adopting efficient mechanisms of production that reject the use of inefficient local labor of the developing countries (also see Waverley 2007, IDRC 2007).

The IDRC argues that tobacco production actually increases unemployment and poverty rates in the developing countries because the companies pay the farmers meager incomes insufficient to cater for the needs of the employees. The tobacco companies also use youth addiction as a means of sustaining their businesses and its epidemic (WHO 2008). The World Health Organization (2008) argues that the tobacco companies adopt the youth as "replacement smokers" because the companies know that once the young get addicted to smoking at an early age, there is a high tendency for them to remain smoking for the rest of their lives. According to the RITC (2006), the actions of the tobacco companies have resulted in a higher consumption of cigarettes and other tobacco products in the developing countries, with its accompanying health 
hazards and deaths. From the 1970s to the early 1990s -around the same period the developed countries saw a reduction in the consumption of cigarettes - the developing countries witnessed an increase of over $65 \%$ in consumption rate, which raises serious concerns (Frey 1997; Jha and Chaloupka 2000; Sklair 2002; Yach and Bettcher 2000; WHO 1997; World Bank 1999; WCTOH 1994).

The World Health Organization-Framework Convention on Tobacco Control (2009) attributes the increased consumption of tobacco globally to complex factors like trade liberalization, direct foreign investment, global marketing, transnational tobacco advertising, promotion and sponsorship, the international movement of contraband, and counterfeit cigarettes. It is against this backdrop that the Framework Convention on Tobacco Control (FCTC) was established in 2003 to facilitate global adoption of the tobacco control policies. The actors - tobacco control activists, policy entrepreneurs, and epistemic communities - who championed the establishment of the FCTC recognized the importance of a concerted global effort to address the tobacco menace (Roemer and Taylor 2005; Studlar 2004). The effort to address the tobacco problem is beyond the endeavors of individual countries and the actors believe that the establishment of the FCTC as a global umbrella body will help to attract that concerted effort necessary to address the tobacco problem. The FCTC aims at using the adoption of measures, which control the behavior of consumers and the activities of the tobacco companies to minimize the consumption of cigarettes and other tobacco products globally (IDRC 1995; Harry et al 2005; Mamudu 2005; PAHO 2003).

The Framework Convention on Tobacco Control (2009) classifies its provisions into demand reduction measures that control the behavior of consumers and supply reduction measures that regulate the activities of the tobacco companies. The main demand reduction 
measures are contained in articles 6-14 and these are further divided into price and tax measures, and non-price control measures. The price and tax measures focus on the adoption of excise tax on tobacco products to increase the price of the product and compel the youth and marginal income smokers to stop smoking. The non-price measures include: protection against secondhand smoke or "passive smoking", product regulation and ingredient disclosure, the establishment of a national coordinating mechanism for tobacco control, packaging and labeling of tobacco products, education of the public on the consequences of smoking, restrictions on advertising, promotion, and sponsorship, and the inclusion of tobacco cessation services in national health programs.

The main supply reduction measures which are also contained in articles 15-17 are: smuggling or unlawful trade in tobacco product and liability, prohibition of the distribution or sales of tobacco to under age people, the provision of support for economically viable alternatives, and tobacco related research and information dissemination among parties. The first process of the FCTC is the signature stage, whereby the country signs the protocol to become a member. The assumption is that, once a country signs the protocol, it signifies the agreement of the country to ratify the protocol as well as the commitment not to oppose the implementation of the provision by other states (WHO-FCTC 2009). The ratification also comes with binding agreement on the parties to adopt effective tobacco control policies to protect public health and avoid tobacco-related hazards. The treaty came into force 90 days after the $40^{\text {th }}$ country signed and ratified the protocol in 2005 and the treaty currently has 173 members. The FCTC also serves as global norm that indirectly put pressure on countries to comply with the norm expectation because of the core objective of addressing a global health hazard. 
Beyer and Brigden (2003:2) note that reducing the health hazards of tobacco is hard because of the strong addictive properties of nicotine, the "low prices of tobacco products, the well-established social norms, and the constant inducement to smoke, which are fuelled by billions of dollars' worth of tobacco industry advertising and promotion". They assert that one way through which this objective may be achieved is through the use of tax instrument as a tobacco control measure. They further maintain that the raising of taxes on tobacco products and banning their advertising and promotion may help to reduce the use of tobacco if these policies are combined with good information on the associated health risks of tobacco use as well as programs to help existing smokers to quit. Furthermore, they argue that certain countries and jurisdictions have successfully implemented taxation measures to control the consumption of tobacco products, which are considered best practices in the tobacco control regime. For instance, Asare (2009) and Waverley (2007) note that cigarette smoking in South Africa decreased with increases of taxes on the price of cigarette because most of the smokers could not afford the price of the products.

In addition, Laugesen et al (2000) recommend several elements of what can be considered good tax policies for controlling the consumption of tobacco. They identify the tobacco control policies of some developed countries and jurisdictions that qualify as best practices. For instance, they argue that the tobacco control policies of New Zealand have reduced the consumption of tobacco by fifty percent within fifteen years from 1981 to 1996 . They maintain New Zealand was able to achieve the reduction through the adoption of taxation instrument, which was combined with other tobacco control instruments over several years. They indicate that between 1980 and 1994, New Zealand increased its tax revenue on tobacco by three 
times, which decreased adult consumption of tobacco by fifty percent. Moreover, tax rates were also increased in 1995 and 1998.

Laugesen et al (2000:228) argue that cigarettes prices increased by thirteen percent following the 1998 tax increases while sales from shops reduced by ten percent. They maintain that the adoption of a Smoke Free Act in 1990 with the support of a coalition of two hundred and fifty voluntary organizations helped to ban tobacco advertising, restricted smoking in workplaces, and instituted health foundation and sponsorship for sports through taxes instead of tobacco sponsorship. The country also adopted a standardized warning on the tobacco pack, which read "tobacco kills" and was written in both English and in a local language (Maori) to ensure that all groups could read and understand it. In addition, a media campaign to promote total quitting of tobacco use was started in mid-1999. The campaign included the provision of free telephone lines on the packs of cigarettes for people to call. They argue that the combination of these instruments ensured the success of New Zealand's tobacco control measures.

Laugesen et al (2000) also identify other measures for controlling tobacco as giving smokers access to treatment medications. They argue that the U.S. adopted such measures, which proved to be effective for controlling tobacco consumption because it helps existing smokers to quit. They further identify the adoption of restrictive policies to ban all forms of advertisements of tobacco and the surveillance of patterns and consequences of tobacco as other effective measures for controlling the consumption of tobacco. The smoke free policies adopted in some US states such as California were also considered as best practices that are effective for tobacco control. They maintain that the California smoke free policies have ensured smoke free public places that protect nonsmokers from environmental smoke. 
Laugesen et al (2000) also identify litigation, common in the U.S, as one of the means through which the information on the health hazards of tobacco can reach the people through media. They argue that litigation compels tobacco companies to increase the prices of tobacco products to cover the cost of litigation and at the same time forces marginal smokers to quit. Laugesen et al (2000) further identify other measures such as mass media campaigns used in Australia and UK and the policies to reduce youth access championed by the Food and Drug Administration of U.S as other types of the best practices of controlling tobacco. Joossens and Raw (2006) also identify some of the effective practices stressed by the World Bank for controlling tobacco as better consumer information including media coverage, public information campaign, and publicizing research findings. The others are large and direct health warning labels on all cigarette boxes and tobacco products, medical treatment for dependents of smokers who may be suffering from the health hazard from second hand smoke, and access to medication.

In recent time, the World Health Organization (2008) has identified the tobacco control policies of a few other countries as best practices or standardized tobacco control measures. It argues that many countries that laid claim to smoke free laws, from limits to bans have subjected nonsmokers to environmental tobacco smoke because their laws did not provide a smoke free environment. The World Health Organization further argues that the effective practices for controlling tobacco are still emerging and encourages countries to emulate, adopt, and learn practices that are suitable to their policy demands to control the tobacco menace domestically (also see Blanke 2002; CDC 1999; Nathan 2004; Selin and Bol 2002; WCTOH 2000).

Jha and Chaloupka (2000) maintain that the adoption of different tax policies and tobacco control measures will generate different changes in the number of smokers and deaths for 
countries with different income levels. This is exemplified in South Africa where a combination of effective tobacco control policies has helped to decrease tobacco consumption in the country (Asare 2009). Weaver (2000) also calls for the adoption of multiple instruments for controlling tobacco because of problems with measuring the impact of individual ones. Therefore, tobacco control activists recommended the adoption of relevant policies that can reverse the anticipated deaths associated with smoking as soon as possible. This paper asserts that the adoption and implementation of relevant tobacco control measures that have been empirically proven to be effective in controlling the consumption of tobacco in the developed countries will hasten the process and likely achieve the desired results in the developing countries (WCTOH, 2000). The transfer of knowledge will help to ameliorate the problems of legislative and technical expertise that normally accompanies the adoption of new policy initiatives such as tobacco laws (World Bank 1999; WCTOH 2000). Moreover, knowledge transfer effort will be enhanced if the nature of tobacco control in the developing is known, which makes the endeavor of this study important.

Mamadu (2005) argues that the intention of the knowledge transfer is to ensure the convergence of policies that will prevent the tobacco companies from taking advantage of weak laws in the developing countries to spread tobacco related health hazards through the promotion and consumption of their products. Moreover, countries are encouraged to institute policies that are tougher than the general requirement recommended by the FCTC (Framework Convention Alliance, 2001). The countries are also implored to adjust the measures to suit their policy situations and economic structure as well as the level of relevance of the policies to the countries (World Bank 1999). This is because innovations that do not conform to local beliefs and values may not succeed (Berry 2008, 9). 
Table 1.5: Relevance of Tobacco Control Policies to Countries of Different Income Levels

\begin{tabular}{llcc}
\hline Policy instrument & Low-income & Middle-income & High-income \\
\hline Raise tax & 3 & 3 & 3 \\
Research causes, consequences and costs & 2 & 2 & 2 \\
Mass information, prominent warning labels & 3 & 3 & 2 \\
Ban advertising and promotion & 3 & 3 & 3 \\
Restrict public and workplace smoking & 2 & 2 & 3 \\
De-regulate nicotine replacement products & 1 & 2 & 2 \\
Control smuggling & 3 & 3 & 3 \\
\hline
\end{tabular}

$3=$ highly relevant, $2=$ relevant, $1=$ somewhat relevant, $0=$ not relevant.

Source: Table adopted from the World Bank, 1999

Hence, the World Bank (1999) identifies different tobacco control policies that are relevant for different countries based on their income levels (see Table 1.5 above). It is presumed that having a clear idea of the structure of the tobacco control processes in the developing control will aid the countries to borrow the requisite knowledge necessary to counter the activities of the tobacco industry network in their area. Berry and Berry (2007: 177) and Collier and Messick (1975) identify developed countries, especially the US, as pace setters in social security policy, which countries - both developed and developing- emulated. Studlar (1999:72) also argues that the Seventh World Conference on Tobacco or Health (WCTOH) reinforced the claim of the Canadians to be world leader in tobacco control, based on substantial increases in taxes on tobacco products and the enacting of the Tobacco Products Control Act by the federal government in 1988 . 
Studlar (1999) further notes that the Canadian government was recognized by the WCTOH in the 1990s for its leadership in improving the health of Canadians and also for the outstanding example shown by adopting a comprehensive tobacco control policy. Therefore, the adoption of tobacco control policies that have been effectively implemented in the developed countries, especially US, Canada and UK could make it easier for developing countries to tackle the tobacco menace in their individual countries with little adjustment. This will save the time and resources that would have been used if countries were to formulate their own domesticallydeveloped control policies. It will also help the developing countries to minimize the strategy of the tobacco industry to undermine the adoption of stricter control measures as domestic policies even though such measures have benefits of saving the lives of millions of people.

Interestingly, a handful of reports suggests that some governmental and nongovernmental actors from the developed countries are contacting some developing countries to transfer knowledge on the best practices of tobacco control for the adoption of domestic tobacco control laws (PAHO, 2003; PHASA, 2007; WHO- EMRO, 2001; WHO-AFRO, 2000; WHO 2005, RITC 2006, IDRC 2003, The Commonwealth Secretariat 2004). The reports talk about conferences and meetings organized to deliberate on measures to assist the developing countries with funding and programs that will enable them to adopt domestic tobacco laws. The reports indicate that the intention of the meeting was to address the challenges posed by increased tobacco production and consumption, especially to the economic development of the developing countries. The contacts also aim at preventing massive tobacco related deaths. For instance, Research for International Tobacco Control (RITC 2006:2) reports on a meeting held with the stakeholders "to prepare a multidisciplinary regional agenda for tobacco control research, to 
promote adequate funding for research, and build a strong knowledge base for effective public policy in Africa."

The Research for International Tobacco Control (2006:5) notes that the meeting was organized for East, Central and Southern Africa, and it was held in Zimbabwe to launch a local initiative for research that focuses on sound regional research priorities. The report further indicates that the objective of the meeting was to address the problem of tobacco production and consumption globally to attract more funding for policy-oriented tobacco control research. It also aims at building capacity to conduct policy-oriented research and to provide policy makers with sound global tobacco control data that will support the Framework Convention. It notes that similar meetings were organized in the Latin American and Caribbean as well as the Asian regions. The World Health Organization - Regional Office for Africa's (WHO-AFRO, 2007) examines the collaboration between the WHO, CDC, and the coordinators of 17 African countries to survey the impact of tobacco on the youth of Africa. The survey result was to serve as a guide for control measures in the region.

The World Health Organization- Regional Office for Africa in its 2000 report examines an inter-country meeting organized by the WHO, CDC, the World Bank, Organization of Africa Unity (OAU) (now the African Union, AU), and the government of Kenya on tobacco control policy and programming in Nairobi, Kenya. The objective of the meeting, according to the report, was to assist policy makers in the Africa region to institute appropriate tobacco control measures to curtail the harm of tobacco on the African people. The meeting also discussed issues of legislation, policy campaigns, and educational programs for tobacco control.

A report by the World Health Organization- Eastern Mediterranean Regional Office (WHO-EMRO, 2001) identifies the measures taken by the WHO to assist with the dissemination 
of information and also to educate the people about the health hazards of tobacco in Cairo, Egypt. The report notes that the WHO brought experts to share their expertise with members at the meetings and the media on educational programs for the public. The Pan American Health Organization (2003) looks at what the WHO through the FCTC is doing to assist national governments to improve the health of people within their jurisdictions. It also looks at attempts to transfer knowledge among countries and focuses on the role of key actors in the tobacco control regime.

Lando et al (2005) examine the institutional and governmental support being offered to establish tobacco research and capacity building programs globally. They identify the efforts of the Fogarty International Center of the National Institute of Health Research, the CDC, the Canadian Institute of Health Research, and the WHO Tobacco Free Initiative as key actors collaborating support for the program. John Hopkins and the London School of Tropical Disease are some of the other institutions that undertake extensive research on tobacco. For instance, the John Hopkins University has an Institute for Global Tobacco Control that generates, synthesize, and translate scientific evidence to support effective tobacco control programs, activities, and policies as a way of preventing the epidemic caused by tobacco. The institute works with over 40 countries around the world to promote global tobacco control.

The London School of Tropical Disease also undertakes extensive research aimed at improving national and international public health policies and health in general. The Fogarty International Center is an institution within the US National Institutes of Health. The Center supports and facilitates global health research conducted by U.S. and international investigators. It also helps to build partnerships with health research institutions in the U.S. and abroad, and trains the next generation of scientists to address global health needs. With regard to tobacco 
control, Lando et al (2005:939) argue that the Fogarty International Center provides funding to support both trans-disciplinary research, including basic tobacco science and research involving partnerships between primarily US scientists and scientists in the developing countries. Lando et al (2005) state that the goal of the Center is to reduce the burden of tobacco consumption in lowand middle-income countries by conducting observational, interventional, and policy research of local relevance and to build capacity in epidemiological and behavioral research, prevention, treatment, communications, health services, and policy research. The Canadian Institutes of Health Research (CIHR) is a government agency responsible for funding health research in Canada. The Institute supports global tobacco research, including the Canadian Tobacco Control Research Initiative (Lando et al 2005).

According to the World Health Organization, the Tobacco Free Initiative (TFI) is an institution established under its auspices to focus on international attention, resources, and action on the global tobacco epidemic. The TFI was established in July 1998 to develop and implement the FCTC protocol and it works jointly with the six regional offices of the WHO to promote global tobacco control (Interview 2011; WHO 2005; 2009). The TFI has regional advisors for tobacco control for each of the WHO regional offices who work closely with the WHO country representatives and national counterparts for tobacco control. The TFI staff at the headquarters in Geneva works closely with its regional advisors and staff in regional offices to plan and implement all activities.

The World Health Organization (2009) maintains that the core objective of the Tobacco Free Initiative is to reduce the global burden of disease and death caused by tobacco. It also seeks to protect present and future generations from the devastating health, social, environmental and economic consequences of tobacco consumption and exposure to tobacco smoke. It provides 
global policy leadership, encourages mobilization at all levels of society, and promotes adoption of the WHO-FCTC protocol. The Tobacco Free Initiative also encourages countries to adhere to its principles, and supports them in their efforts to implement tobacco control measures based on its provisions. Lando et al (2005) argue that the Tobacco Free Initiative supports communication, media, and advocacy for policy change. It works with countries to strengthen national capacity for the development of sustainable tobacco control activities and also participates with collaborating centers and other international agencies to promote regional and country tobacco control work as part of its global network function (WHO 2005; 2009).

Another core function of the Tobacco Free Initiative is the surveillance and monitoring program administered jointly with the US Centers for Disease Control and Prevention. The collaboration between the two organizations has led to the establishment of the global tobacco surveillance system, including the Global Youth Tobacco Survey, the Global School Personnel Survey and the Global Health Professionals Survey, that enables TFI to monitor and evaluate international tobacco related issues by reviewing structural elements (task forces, commissions, nongovernmental organizations), process developments (laws and regulations, economics, behavior, exposure, advocacy) and epidemiological data (prevalence, morbidity, mortality) (WHO 2005).

As a result, the regional offices of the WHO-TFI has established comprehensive databases on the use of tobacco products and national tobacco control policies in their respective countries and implemented a global information system for tobacco control as well as instituted a system to monitor, report, research, and analyze the activities of the tobacco industry (WHO 2005). The TFI also works with an international network of scientists and health experts to promote research on various aspects of tobacco production and consumption and their impact on 
health, economics and environment. It also develops and disseminates policy recommendations on tobacco control instruments and measures, including issues and policies on second hand tobacco smoke, tobacco and youth, gender responsive tobacco control, smoking cessation, liability and litigation, indigenous population and tobacco control, and tobacco and tuberculosis (WHO 2005; 2009; Interview 2011).

The Public Health Association of South Africa (PHASA, 2007) presents information on a workshop collaboratively organized by the WHO, the RITC/IDRC, the Physicians for a Smoke Free Canada, and government officials of some Eastern and Southern African countries to provide leadership in tobacco control in Africa. It describes the workshop as having stressed the intention to generate sound scientific data and knowledge to aid the adoption of the FCTC measures. The Research for International Tobacco Control (RITC) and the International Development Research Center (2007) identify the support offered by RITC to the University of Cape Town (UCT) and other local NGOs in South Africa in 1996 to conduct a comprehensive study on the economics of tobacco control in South Africa and the African region.

Unfortunately, these reports fail to present an in-depth academic and theoretical analysis of the specific domestic laws being adopted by the developing countries and the political struggles and dynamics that occur during the transfer of knowledge and ideas in the policy adoption processes. The preliminary analysis above clearly shows that the tobacco companies and their affiliates are using different strategies to prevent the adoption of tobacco measures in the developing countries. Simultaneously, the anti-tobacco activists are speeding up the attempt to control tobacco through knowledge transfer. Therefore, the study analyzes the two streams of propaganda and elucidates the nature of political struggles as a way of contributing to literature. In particular, knowledge is added by shedding light on how the processes work in the 
individual countries and also by illustrating how the global advocacy network, liberalconstructivist theory, and knowledge transfer frameworks could be used within a single framework to understand the process of policy change. By providing a detailed report on the collaborative global effort to assist the developing countries with the control of tobacco consumption and its related menace, this study adds to studies that implore social constructivist, advocacy network, and policy transfer theories to explain policy change. Lastly, the study contributes to the political science, public policy, comparative public policy and international relations literature.

\section{Organization of the Dissertation}

Chapter Two presents the theoretical framework outlining and discussing the various theories for the study. It also looks at the methodology, data, variables, and the specific research questions for this study. Chapter Three examines the operations of trans-border advocacy networks, focusing on their goals, motivations and the strategies that are employed to influence their target actors. It also analyzes the structure of policy change in the Africa region and the anticipated policy change in the developing countries. Chapter Four examines tobacco control activities of Ghana and tobacco control instruments adopted in the country as well as the political struggles and resistance that occur during the process. It also looks at the composition of actors who are directly or indirectly involved in the process. It analyzes the data collected from the interviews and secondary documents to understand the nature of tobacco control in Ghana. Chapter Five does the same analysis as in Chapter Four for Malawi and it assesses the tobacco instruments adopted by the country and the inherent political struggles. 
Chapter Six discusses the findings and evaluates how well the theories adopted for the study have been able to provide understanding of the processes of knowledge transfer that are occurring between the developed and developing countries. It compares the different domestic laws adopted in each of the countries and examines the similarities and difference in the factors that facilitated the adoption of the laws. It also describes the political struggles that occur between groups affiliated to the tobacco industry and the tobacco control activists. Finally, the chapter assesses how the nature of bureaucracy of the countries impact the adoption of the voluntary agreements and why Ghana has made more progress than Malawi in the adoption of voluntary agreements as tobacco control instruments and also presents the conclusion of the study as well as makes suggestion for future research. 


\section{Chapter Two}

\section{Theoretical Framework}

\section{Introduction}

This chapter explains the theoretical framework for the study. It examines the transborder advocacy network approach, which is used to investigate how the best practices of tobacco control are adopted as domestic laws in Ghana and Malawi. The trans-border advocacy network approach combines three theories: the multi-level policy transfer approaches, the global advocacy network approach, and the liberal-constructivist approach to international relations. These theories are complementary to understanding the adoption of tobacco control policies in the selected countries because all three theories attempts to explain how and why policy changes occurs. They focus on the role of policy actors and/or ideas in influencing the adoption of policy.

The central focus of the tobacco control knowledge transfer leading to the adoption of tobacco law by the developing countries is on how states change their behavior in the international system. Generally, the preferences of states are shaped by the interactions between transnational actors in favor of a global regime of tobacco control (Mamudu 2005). Therefore, the theories collectively contribute to the understanding of the processes that influence countries to change their behavior. A core issue that often arises with the analysis of the factors that cause countries to adopt certain ideas propagated by transnational actors as domestic policy, is the question of the levels of analysis that help to explain the process. Caporaso (1997) maintains that the issue of levels of analysis and the research associated with it often leads to an ontological question centering on the appropriate level to analyze the forces behind the adoption of certain policy decisions, especially when the idea behind the decision is propagated by transnational actors (Mamudu 2005). 
Often addressing such a question entails a consideration of major theories under various levels of analysis. The tobacco control knowledge is transferred by a combination of actors comprising individuals, interest groups, bureaucrats, intergovernmental and nongovernmental organizations who collaborate to promote conformity with the FCTC norm. Therefore, the study involves multiple level of analysis and an assessment of the various theories to understand the phenomenon. The combination of the theories adopted for the research will help to address the problem of the level of analysis, and explain the ratification of the FCTC and the adoption of tobacco control law to ensure compliance with the FCTC convention (Mamudu 2005). As earlier indicated in Chapter One, the tobacco control knowledge occurring in the developing countries involves institutions, interest and ideas.

The multi-level knowledge transfer approaches explains how voluntary and indirect coercive means - involving the use of incentives - are used to engage bureaucratic institutions such as the Ministry of Health and interest groups to promote tobacco control ideas. The global advocacy network helps to identify and explain the activities of the actors who are engaging governmental institutions and interest groups to promote their interest as tobacco control ideas for adoption. Lastly, the liberal-constructivist approach explains why the receiving countries are adopting or not adopting the ideas being disseminated. The chapter starts with a general discussion of the theories and how they relate to the adoption of best practices of tobacco control as domestic laws. Then, it presents the specific research questions, the variables, and the methodology outlining the procedure of the research and data collection. The diagram below depicts the conception of this study on how the three theories facilitate policy change. 


\section{Figure 2.1: Knowledge Transfer Diagram}

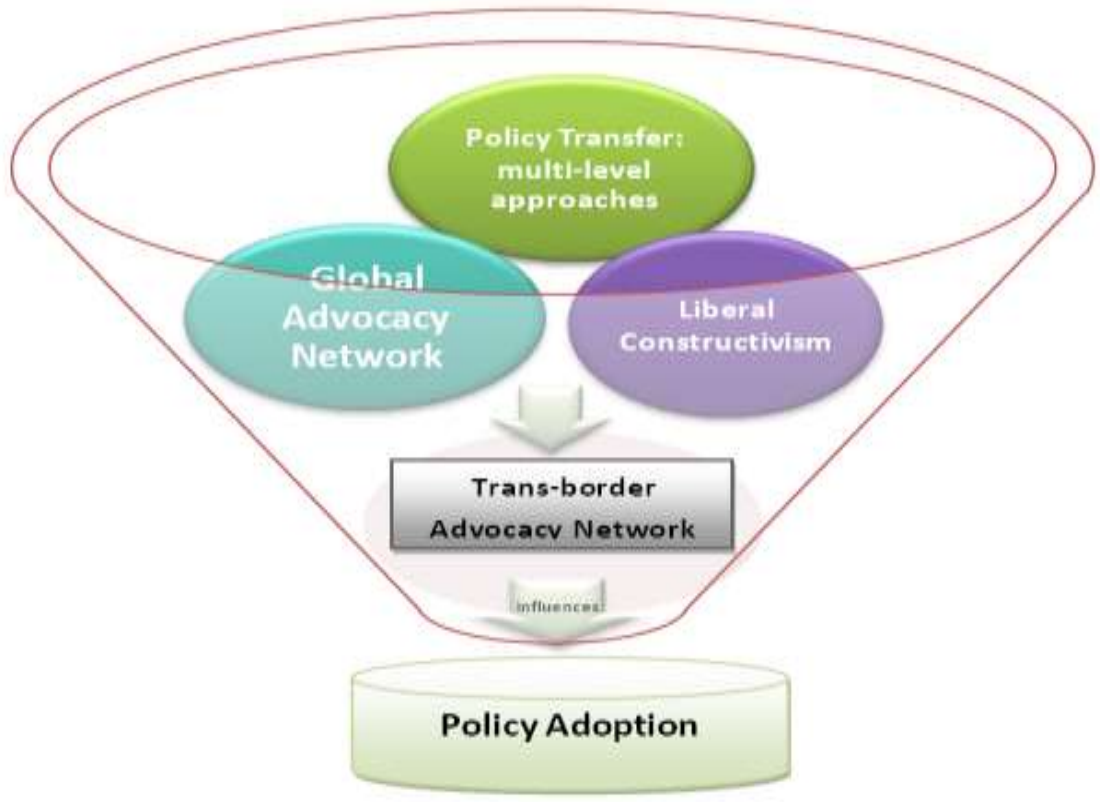

Source: (Based on information from the studies of Farquharson 2003, Finnemore 1996, Mamudu 2005, Dolowitz and Marsh 2000, Evans and Davies 1999, Evans 2004, Ladi 2005, Wendt 1992, Gidden 1996)

\section{Knowledge Transfer}

Scholars have treated knowledge transfer under different names such as policy diffusion, emulation, policy copying, and bandwagon, among others. Since the classic works of Walker (1969), Crain (1966), and Gray (1973) explaining innovation and policy transfer among units of governments, numerous studies have emerged in the political science and public policy literature on policy transfer. Evans (2004:10) defines policy transfer "as a theory of policy development that seeks to make sense of a process or set of processes in which knowledge about institutions, policies or delivery systems at one sector or level of government is used in the development of different institutions, policies or delivery systems at another sector or level of governance”. He classifies the policy transfer literature into voluntary or lesson drawing, negotiated/indirect coercive policy transfer, and coercive policy transfer. However, Studlar (2002:81) observes that 
the concepts involve learning from others, either horizontally or vertically by governmental actors or political jurisdictions in the policy process. The interest of this study is to examine how voluntary and negotiation policy transfer theory can be used to explain the processes of policy change occurring in the area of tobacco control policies of the developing countries. Specifically, the theory is used to examine how bureaucratic institutions such as the public health departments which have been the main agent of tobacco control in the developing countries are being influenced to adopt tobacco control instruments in Ghana and Malawi.

\section{Lesson Drawing / Voluntary Policy Transfer}

To Evans (2004), lesson drawing is a voluntary form of policy transfer involving a rational and calculated decision to use policies implemented elsewhere or ideas there from, to address public policy problems in a different jurisdiction. He notes that the voluntary policy transfer can occur if the public or professionals identify dissatisfaction with an existing policy because of poor performance or if a new government, minister, or management decides to introduce a new policy agenda. He further argues that a decision of policy officials to act on a decision that has been previously agreed on could bring about a voluntary policy transfer. He asserts that a jurisdiction can adopt a voluntary policy transfer to strengthen relations with political allies or to defuse political enemies.

Evans (2004) further notes that lesson drawing is a different process of policy transfer in which adopting countries willingly borrow policy ideas to address their problems. To Newmark (2002:152), lesson drawing is a specific form of policy transfer. He further describes policy transfer as another form of policy diffusion. Graham et al (2008:3) maintain that diffusion occurs when the decision of one government about whether to adopt a policy innovation is influenced 
by other governments' previous choices. In other words, observing what others have done can influence another country or jurisdiction to tailor its own policy on the policy observed. Mostly the actors drawing lessons examines the policies and programs of other countries to ascertain how the countries solved similar policy problems in the past (Evans 2004). Additionally, the actors drawing lesson compare the content of a policy and the manner through which such policy information is communicated (Studlar 1999:69). This enables the adopting countries to borrow the experience from other countries to solve their policy problems (Rose 1991, Bennett 1991). Studlar (1999) maintains that lesson drawing studies qualitatively analyze how policies are transferred from one jurisdiction to another.

Rose (1991) argues that the desire of policymakers to find solutions to problems within their jurisdiction brings about lesson drawing. Also, Newmark (2002:154) and Robertson (1991) argue that lesson drawing enables the policy makers to manipulate the policy process. They claim that the policy makers through lesson drawing take a short cut to the policy process of solving a problem to avoid reinventing the wheel, especially in situations where other countries had already addressed a similar problem. To Asare (2007:52), lesson drawing culminates in a situation whereby jurisdictions copy and apply the policies of others in their home countries, with the goal of achieving similar outcomes as the jurisdictions where the policies were copied. This study is interested in adopted policies aimed at achieving similar outcomes of tobacco control in Ghana and Malawi as the countries where the policies initially implemented. Nevertheless, the study also pays attention to learning from the mistakes of the developed countries and adopting a policy that is completely different from the one learnt. Sometimes, the countries drawing lessons from other countries could end up with a completely different policy because they did not understand the details of the policy. This can result in negative 
consequences whereby the policy adopted turns out to be completely different and more likely fails to address the policy problem it was intended to address (Evans 2004; Studlar 2002). The study also acknowledges the differences in the developing countries that may call for different policy outcomes.

Newmark (2002) identifies diffusion as a general term, which often involves situations whereby structural and modernizing factors in the external environment necessitate the adoption of a domestic policy. To Studlar (1999:69) diffusion when "broadly considered, is oriented more toward categories of policies, outcomes, and the adoption consequences" than lesson drawing. Moreover, Newmark (2002) notes that policy diffusion could take different forms, including lesson drawing. Graham et al (2008:4) argue that diffusion occurs when policies adopted by some countries influence another country to reform its existing policy or to adopt a new policy. Therefore, lesson drawing and policy diffusion revolve around access to information on policies earlier adopted by other countries. The access to such information helps the countries to hasten the process of addressing domestic policy problems. Access to relevant policy information can also save time and resources and prevent the repetition of previous mistakes (Graham et al 2008).

Mintrom and Mossberger (2008:3) consider both diffusion and policy transfer as forms of policy change generated by new ideas, interests, and information. They argue that the spread of new information and ideas promotes new policy interest, which brings about demand for policy change. However, new interests can also generate new policy ideas and information. For instance, the ideas to control tobacco has generated new policy interests, some of which include adopting domestic laws to protect nonsmokers from environmental smoke, restricting the advertisement of tobacco by the tobacco companies, or placing excise taxes on cigarette to curtail smoking. Moreover, the interests to curtail the epidemic associated with the consumption 
of tobacco have significantly influenced the emergence of these policy ideas. The motive underlying the knowledge transfer in the tobacco control regime is for the adopting countries to use the experiences of the developed and other leading regional tobacco control countries to institute intervention measures that will halt the tobacco epidemic at this early stage (Lopez et al 1994).

\section{Negotiated / Indirect Coercive Policy Transfer}

Evans (2004) identifies negotiated policy transfer as another means through which policy transfer occurs. He classified the negotiated policy transfer into two, both of which involve the use of some sort of coercion. The first is when global financial institutions, transnational corporations, international organizations, donor countries, or supra-national institutions use the adoption of certain policies as a condition for obtaining grants, loans, and other forms of investments. This is comparable to what Graham et al (2008) identify as a vertical form of coercion whereby nongovernmental and international governmental actors use indirect pressure on governments for them to adopt certain policies (also see Walker 1969; Gray 1973; Berry and Berry 2007). They argue that in the vertical form of coercion, intergovernmental actors compel governments that have not adopted certain policy reforms to adopt their preferences. An example of such strategy was the International Monetary Fund's requirement in the past for developing countries to adopt the structural adjustment programs (Evans 2004: 3). Evans (2004) notes that the policy was based on western conception of good governance and was used as conditions for receiving loans and grants.

In the tobacco control regime, this form of coercion is comparable to the condition/policy of the World Bank Group, which prohibits the use of its funding to undertake 
activities that promote tobacco consumption (Asare 2007:13). Asare (2007) maintains that the policy of the World Bank Group is to promote tobacco control, arguing that the policy indirectly compels countries to adopt tobacco control policies as condition for loans and grants. Mintrom and Mossberger (2008:3) attribute the ability of an entity to pressure another entity to adopt a certain policy to power. They maintain that power enables actors to impose their ideas or preferences as condition for aid and grants, or for interest groups and policy entrepreneurs to promote their interests. They further argue that although ideas may spread through political processes, power is actually the most influential actor for the spread of ideas. Therefore, this study anticipates that the adoption of domestic tobacco laws in the developing countries is as a result of the economic power of financial and other forms of incentives offered to the adopting countries by countries like the UK, US, Canada, intergovernmental, and nongovernmental organizations in the developed countries.

The tobacco control actors in the developed world are using their economic power indirectly to coerce the adoption of their preferred best practices of tobacco control as laws in the developing countries, and this is done through the use of aids, grants, and other means. Particularly, given that the adoption of the voluntary agreements is mostly fronted by the governmental institutions such as the Ministry of Health which also receive a lot of their operating budget from aids and grants provided by the WHO for disease control, the study anticipates that some of the funds are used as indirect means of coercing the adoption of the tobacco control instruments. In that sense, the importance of economic aid to survival of the operations of many of these governmental institutions may compel them to kowtow to the demands of the tobacco control knowledge transfer agents. Alternatively, many of the 
developing countries who feel pressured to adopt the policies against their will may refuse to adopt the recommended policies.

Evans (2004) also notes that competition and the fear of falling behind neighboring countries can indirectly force some countries to adopt certain policies (also see Graham et al 2008). Graham et al (2008) maintains that competition compels governments to adopt policies that will make them more attractive to investors and their citizens. To Mintrom and Mossberger (2008), new ideas can generate competition among neighboring countries and put pressure on some countries to emulate earlier innovators. They maintain that the force inducing the pressure to use new ideas for solving domestic public policy problems could be from either the external or internal environment. Berry and Berry (2007: 171) and Walker (1969) argue that sometimes jurisdictions compete to adopt policies earlier adopted by others when such policies are attractive to a vocal minority or majority of their citizens. They note that in other instances, countries adopt policies considered as accepted standard in the region or globally to avoid being considered a slacker or awkward (also see Drezner 2001).

Drezner (2001) further notes the force compelling the countries to adopt new policies or to change their institutions and regulations could be a strong belief or idea that the adoption of the policy is the right action to take. In the politics of tobacco control, the illustration of competition to conform is the pressure to adopt the globally accepted best practices of tobacco control by the developing countries. The belief that tobacco consumption is linked to global health hazards is so strong that the countries feel pressured to conform to the norm expectation set by the tobacco prohibition regime by adopting the control measures as domestic laws. The prohibition regime is the process whereby certain actors internationalized their beliefs on certain issues as a global norm for other countries to adopt (Nadelmann 1990). The belief about the need 
to protect global public in this case becomes the norm that has been internationalized for controlling tobacco, and countries are pressured to adopt measures to protect their citizens and also because of the fear that failure to do so may make them a straggler. In addition, actors who share the beliefs about the health hazards of tobacco disseminate information and form alliances with local advocates in a way that it generates domestic pressure for the government to adopt tobacco control legislations. Therefore, it is expected that the pressure from external and domestic tobacco control activists will compel Ghana and Malawi to adopt domestic laws.

Evans (2004) identifies another type of policy transfer as direct coercive policy transfer, which entails the use of force by one government on another to adopt certain policies against the will of the country. Graham et al (2008) identify direct coercive policy transfer as the horizontal type of coercion in which certain governments pressure others to adopt policies earlier adopted by the former. For example, Britain compelled her colonies to adopt policies of the mainland during the colonial period. However, this study does not see the feasibility of a direct coercive policy transfer in the tobacco control policy process. Moreover, the strengthening of notion of sovereignty makes it theoretical impossible to anticipate a direct coercive policy transfer in modern times. Therefore, the study focuses on the voluntary and the negotiated policy transfer. It examines how the countries are willingly adopting tobacco control policies of their choice. Simultaneously, it examines how governmental and nongovernmental organizations are using incentives to coerce the adoption of their preferred tobacco control policies in both countries.

Even though the modern power relations and control facilitated by economic strength and the structure of the United Nations ${ }^{12}$ may suggest the feasibility of a direct coercive policy

\footnotetext{
${ }^{12}$ The structure of the U.N offer more powers to the members of the Security Council and the economically powerful countries of the world feel it is their moral responsibility to prevent epidemic of any type globally. Therefore they can exert pressure on certain countries against the will of the country when they believe that such action will avoid an epidemic or promote peace.
} 
transfer, the study believes that such coercion can only take place through negotiated or indirect means. In other words, the economically advanced countries such as U.S, Canada, U.K, and related actors may only indirectly use incentives to compel the developing countries to adopt the tobacco control policies that have helped to reduce tobacco consumption and its hazards in those countries. Therefore, the study examines how Ghana and Malawi are adopting ideas on the best practices to control tobacco domestically because of incentives provided for that purpose. It also investigates how the ideas about the health hazards of tobacco consumption are putting pressure on the countries to adopt the tobacco control measures. In addition, it examines the negotiations that sometimes occur between the tobacco companies and the countries on the types of laws to be adopted that are acceptable to the companies.

Some scholars maintain that policy transfer is induced by similarities in culture, historical experiences, ideology, common language, or institutions (Castles 1993; Leichter 1983; Studlar 2002, Peters 1997, 1998), geographical proximity (Walker 1969; Gray 1973), modernizing factors (Wolman 1992), the spread of new system of ideas (Hall 1993; Stone 1996, 1999, 2000, Evans and Davies 1999; Ladi 2005), and the economic status of countries (Rose 2005; Berry and Berry 1999, 2007; Berry 2008). The studies focusing on the similarities in culture, ideology, and institutions normally espouse common historical legacies, similar religious and cultural experiences, and the use of common language among the elites of the countries, as the motivating factor for the knowledge transfer among countries. For instance, Castles (1993) identifies the "family of nations" as a motivating factor for knowledge transfer. His analysis focuses on common religious traditions, cultural practices, and historical ties.

Mintrom and Mossberger (2008) argue that some countries concentrate on certain policy innovations but ignore others, based on their common historical ties. To Newmark (2002) and 
Dolowitz (1997, 1998), actors normally become aware of policies adopted by other countries through media reports, conferences and papers, or direct contact with the officials of the countries that had already adopted the policy. However, the adoption of policies by the country is mostly influenced by ideology or common language. They also maintain that activists or transnational actors who see the adoption of a particular policy as the best means for addressing a societal problem can form coalitions to institute a prohibition regime necessary to influence the adoption of such policy (Newmark 2002; Castles 1993; Dolowitz et al 1999; Graham et al 2008; Berry and Berry 2007; Nadelmann 1990).

Therefore, this paper examines how common historical ties or the "family of nations" concept serves as one of the explanatory variables in understanding the process of tobacco control knowledge transfer from the developed to the developing countries. It focuses on the common use of the English language among the elites of the members of the Commonwealth and the activities of the transnational actors within the Commonwealth to promote the adoption of tobacco control legislation as the best means of solving the tobacco epidemic. Leichter (1983: 224) argues that membership of the Commonwealth facilitates contacts, interactions, and broad communication among members. He notes that the common use of the English language among the elites, a legacy from British colonial rule, facilitates extensive communications among the members as well as non-members where the elites use the English language. For instance, the meetings of the Health Ministers of the Commonwealth have become venues through which member countries deliberate on measures to control tobacco in their respective countries (Krishnan et al 2003).

The Commonwealth countries witness enormous amounts of the activities of the tobacco industry because the second largest transnational tobacco company in the world (British 
American Tobacco) and other companies like Phillip Morris have affiliates in many of the Commonwealth countries (Krishnan et al 2003). The Commonwealth is also made up of countries like Canada, Australia, Malaysia and South Africa that have comprehensive tobacco control policies and those that have voluntary agreements that are not properly enforced. This provides incentives for knowledge transfer, especially due to the history of cooperation on policy issues among members of the Commonwealth (Leichter 1983).

For instance, Leichter (1983) focuses on common historical tiers and the adoption of policies among Commonwealth countries and argues that the Commonwealth normally encourages members to adopt uniform policies in areas where cooperation is relevant. The tobacco control issue is an area where cooperation is needed because of its global impact on public health. Therefore, tobacco control is considered as an area where the Commonwealth and other relevant international governmental institutions could encourage cooperation and the adoption of uniform tobacco control policies by member countries and the broad interactions between the members would facilitate the adoption. However, the presence of the headquarters of British American Tobacco in the UK, which has affiliates in almost all the member countries of the Commonwealth, can serve as a strong incentive not to adopt comprehensive tobacco control laws. This is because the officials of BAT may lobby Commonwealth and governmental officials to protect their economic interest. Therefore, examining the tobacco control activities of the Commonwealth may shed light on the politics and process of transferring the FCTC measures, which are adopted in the developing countries.

Berry and Berry $(2007,172)$ maintain that generally each contact between officials of prospective adopters of a policy with previous adopters increases the chance of a policy being adopted. However, in some instances, the contact can lead to the contrary, especially when 
contradictory information is discovered through the contact or when it generates local political resistance to prevent the adoption of a particular policy from another country or jurisdiction. Leichter (1983) also contends that the Commonwealth institutions provide "institutional venues" that ensure broad interactions and movements among officials and people to share ideas and knowledge on certain public policy issues. Therefore, the Commonwealth could serve as the institutional venues for the transfer of knowledge on effective tobacco control measures.

Institutional venues for tobacco control are places where tobacco control governmental and nongovernmental actors meet to deliberate on issues of common interest (Mamudu 2005). Other examples of institutional venues are the conferences of the WHO, the World Conference on Tobacco or Health, and the United Nations. Mamudu (2005) identifies the conferences of the World Conference on Tobacco or Health (WCTOH) as one of the institutional venues where the officials and tobacco control activists meet to deliberate on the best practices of the tobacco control. Contacts made through such venues normally generate other formal and informal follow ups in the form of workshops to provide training, capacity building for research, internet/email correspondence, introduction to essential literature on tobacco hazards, awareness creation and educational skills as well as longer time mentoring of civil servants and advocates ( Interview 2010).

Evans (2004) argues that knowledge can be transferred from all countries, irrespective of the level of development or economic status. He maintains that knowledge can be transferred from one developed country to the other, from developed to developing countries, from developing to developing countries, or from developing to developed countries. Therefore, the current study also examines the role played by other regional countries that have adopted comprehensive tobacco control laws in the knowledge transfer processes. In particular, it 
examines the role of South Africa in influencing other developing countries of the Commonwealth to adopt tobacco control instruments. Other scholars have observed that countries normally adopt policies from the economically advanced countries (Rose 2005; Berry and Berry 1999, 2007; Berry 2008). Leichter (1983) argues that sometimes resource constraints compel the less developed countries to borrow policies from the developed countries.

In the case of the Commonwealth, Leichter (1983:228) and Collier and Messick (1975) found that most of the time when the reasons for knowledge transfer involve expertise on legislative, technical, and administrative issues, the less developed members of the Commonwealth normally seek substantive and technical guidance from the developed members. It cannot be denied that the tobacco control problem confronting the developing countries requires expertise on technical, legislative, and administrative issues as well as adequate resources to address. Accordingly, it is expected that the leadership and guidance of the developed members of the Commonwealth on such issues will be vital to help the less developed members to adopt the best practices of the tobacco control. At the same time, certain leading regional tobacco control developing countries such as South Africa are also expected to be part of the coalition pushing for the adoption of effective tobacco control through the Commonwealth.

The presumption is that the developing countries will adopt knowledge from the developed members not only because of resource constraints to deal with the tobacco problem but also because of the experience of U.S, Canada, and UK with tobacco control (Leichter, 1983). Such process could occur through socialization because those countries have held themselves as attractive and prominent leaders in the tobacco control regime worthy of emulation (Graham et al 2008). Moreover, the other actors of the tobacco control knowledge 
transfer like the WHO, the American Cancer Society, the Bloomberg Initiative and Foundation, the Bill and Melinda Gates Foundation, IDRC, among others, and the epistemic communities are expected to facilitate the diffusion of the norms as a way of socializing the developing countries into the community of countries that have best practices of tobacco control (Adler 1992, Haas 1992, Graham et al 2008, Mamudu 2005).

Latif (2004) maintains that the Commonwealth plays a pivotal role in influencing it members to sign and ratify the FCTC protocol. The action of the Commonwealth stems from the belief that the ratification of the protocol is necessary to achieving its millennium development goal of eradicating poverty based on the notion that the ratification will result in the adoption of domestic tobacco control laws to protect public health. The WHO has also been very influential in propagating the notion that the adoption of tobacco control laws will minimize the amount of money spent by the government on tobacco related health hazards and enable the government to concentrate on poverty alleviating programs (WHO 2008). Therefore, the hope is that once a country ratifies the FCTC protocol, the obligations that come with the ratification may influence the country to adopt domestic tobacco control law.

Rose (2005) argues that the officials adopting the knowledge will need to have a detailed grasp of the policy or program being adopted so as to apply it to the problem confronting the domestic policy environment. In other words, policy makers are able to apply the ideas of the policy to their unique situations for achieving intended outcomes when they have full grasp of the policies and programs being adopted (Sabatier and Jenkins-Smith 1999; Wolman 1992; Rose 2005). Therefore, the knowledge transfer and learning should enable the adopting countries to acquire the expertise needed to use local initiatives modeled after the best practices of the 
tobacco control to address domestic problems (Newmark 1999, 2002; Dolowitz 1998; Schneider and Ingram 1988; Rogers 1995; Berry 2008).

According to Dolowitz and Marsh (2000:10) bureaucrats and government officials, think tanks, consultants, experts, elected officials, and policy entrepreneurs are the major actors who engage in policy transfer. Political parties, pressure groups, transnational corporations, nongovernmental institutions, and intergovernmental organization also play important roles in policy diffusion and transfer (Dolowitz and Marsh, 2000; Hall, 1993; Sabatier and H. JenkinsSmith 1999; Stone 2004). Studies also indicate that some of the agents of knowledge transfer such as bureaucrats and governmental officials, think tanks, experts, policy entrepreneurs, transnational corporations, nongovernmental organizations, and intergovernmental organizations operate as an advocacy network on issues of the environment, human rights, and women's rights (Keck and Sikkink 1998) as well as tobacco control (Farquharson 2003) to pursue their interest globally. For instance, Studlar (1999) asserts that governmental, nongovernmental organizations and advocacy groups have been instrumental in spreading ideas about tobacco control among different jurisdictions especially in the case of Canada and US.

Graham et al (2008:17) identify three types of actors who perform crucial roles in the policy diffusion process as the internal, external, and go-between actors. They argue that studying the activities and interactions of these actors is crucial to the understanding of the politics that occurs in policy diffusion. Some of the actors mentioned as internal actors are the electorates, elected politicians, appointed bureaucrats, interest groups, and policy advocates who are part of the government considering the policy reforms. They maintain that analyzing the policy environment, objectives, influences, and interests of such actors will help to better understand the diffusion of a particular policy. The external actors, according to the authors, are 
the earlier-decision makers or the governments that had already adopted the policy. Therefore, understanding the motivations and responses of the earlier adopters of the policy will help to give a better grasp of policy transfer.

Graham et al (2008) further argue that the go-between actors are very instrumental in policy transfer from one government to the other, even though they are not part of the internal or the external actors. They identify the members of this group as policy entrepreneurs, epistemic communities, think tanks, academic entrepreneurs, research institutes, mass media, nongovernmental organizations, and intergovernmental organizations who advocate, influence, and facilitate policy reforms based on their individual shared principles and beliefs. In a nutshell, whereas the go-between actors are normally motivated by the shared beliefs and values to influence policy reforms, the internal actors are motivated by the need to address societal problem with public policy by looking at what has been done elsewhere.

Therefore, the internal actors examine the activities of the external actors-the governments that have already addressed a similar problem in order to use it to solve their problem in the domestic environment. The paper examines how the interests and activities of these actors impact the process of tobacco control knowledge transfer, leading to the adoption of domestic policies. Moreover, Alder (1992) and Haas (1992) observe that the policy experts within epistemic communities are also influential in the diffusion of norms. As a result, the study expects that the go-between actors will be vital to the policy transfer and learning of the evidence-based tobacco control policies or best practices (Nathan 2004).

\section{Global Advocacy Network}

The global advocacy network is an approach that helps to describe how the tobacco control actors are promoting their interest as tobacco control ideas for adoption globally. 
The approach focuses on the activities of nongovernmental and intergovernmental actors to influence policy change in the tobacco regime. The nature of knowledge transfer and learning in the tobacco control policy arena occurs through the interactions, information dissemination, and activities of both international and domestic actors of tobacco control. The global advocacy network approach combines significant aspects of theories such as the transnational advocacy network, the advocacy coalition framework, transnationalism, and the epistemic community approaches to understand the activities of the global tobacco control (GTC) (Farquharson 2003; Cairney et al 2012).

Farquharson (2003) identifies the existence of two advocacy networks in the tobacco control regime as the anti-tobacco control network and pro-tobacco control network, and the activities of both networks influence the control of tobacco in the international scene. She maintains that the pro-tobacco advocacy network is made up of actors who are affiliated to the tobacco industry and seek to protect the interest of the industry (also see Cairney et al 2012). The pro-tobacco advocacy network tries to protect the activities of farmers and businesses affiliated to the tobacco industry by preventing the adoption of stricter tobacco control laws, especially in tobacco economies or in areas where tobacco manufacturing plants are located (WHO 2009).

On the other hand, the anti-tobacco network comprises tobacco control actors who want to eradicate the consumption of tobacco products globally. The actors making up the anti- tobacco network are both scientific experts and non-experts. Therefore, the global advocacy network approach is broader than the epistemic community approach, which focuses on a community of experts who share common principled beliefs (Farquharson 2003). Additionally, the approach is different from the advocacy coalition framework 
approach, which emphasizes the domestic policy subsystem as the core characteristics of the coalition seeking to make policy changes (Sabatier and Jenkins-Smith 1999). The tobacco control network comprises both domestic and international actors who are pushing for policy change on tobacco within a certain jurisdiction. Moreover, the global advocacy network adopts certain relevant aspects of the advocacy coalition network.

Farquharson (2003) notes that the global advocacy network is built on a network of advocacy groups with a common shared discourse and belief just like transnational advocacy network actors. However, the two are different because the global advocacy network approach has actors with instrumental goals such as the industry based actors of the pro-tobacco network but the transnational advocacy network excludes them. The global advocacy network has both the actors with instrumental goals and other actors who are motivated by shared principled ideas or values like the IGOs, NGOs, and epistemic communities. Actors with instrumental goals are motivated by profit maximization while those who mobilize on share principles are motivated by their common beliefs and values. The global advocacy network actors operate globally to promote the beliefs of the members through the exchange of services and relevant information. Farquharson (2003) argues that such classification represent the nature of the tobacco control policy subsystem in view of the vital role played by the tobacco industry in the politics of the tobacco control.

The global advocacy network approach is adopted for the analysis of this study because it helps to understand the political struggles that occur during the adoption of domestic tobacco laws in the developing countries. It brings to the fore the activities of the tobacco industry network to prevent the adoption of laws that will hamper their successful operations in the developing countries. In addition, it portrays the strategies and coalitions adopted by the anti- 
tobacco interests and activists to hasten the adoption of best practices of tobacco control as domestic laws in an attempt to halt the tobacco health hazards. The anticipation is that the analysis of the activities of the pro-tobacco and anti-tobacco advocacy networks within the tobacco control policy process will shed light on the success of and impediments to knowledge transfer. One approach that is essential to the tobacco control network is the epistemic communities. The experts examined by the approach use their expertise to provide justification for the adoption of tobacco control ideas. Therefore, the approached is examined below in spite of the fact that it is part of the global advocacy network.

\section{Epistemic Communities}

The impact of issue framing in politics and policy making has been well documented in the political science literature. Framing not only influence the opinion of people but it also determines the actors who play important roles in an issue area (Nelson, Oxley, and Clawson 1997; Kahneman and Tversky 1984; Alder and Haas 1992). Alder and Haas (1992) and Alder (1992) have illustrated how some epistemic communities used expert advice and issue framing to position themselves as core actors in the arms control era. Besides, some epistemic communities have emerged as important actors in the tobacco control regime because of the way that they have framed the tobacco issue (Haas 1992, Mamudu 2005). The tobacco menace has been postulated as a complex global issue that demands expertise and collective global action to tackle. Mamudu (2005:44) identifies the World Conference on Tobacco or Health as one of the tobacco control epistemic communities that have been championing the adoption of best practices at the global level. 
Haas (1992: 3) defines epistemic communities as "a network of professionals with recognized expertise and competence in a particular domain or issue-area". These professionals become indispensable to decision makers when a power vacuum emerges within their area of expertise for which their assistance is required (Edwards 2001, Haas 1992, Alder and Haas 1992). Mamudu (2005:42) argues that what motivates the activities of the epistemic communities is when the authoritative knowledge within the domain of their expertise has to be used to make policy during a power vacuum. The power vacuum results from the condition of uncertainty created by the broad and complex nature of modern problems, which are difficult to address (Haas 1992, Alder and Haas 1992, Edwards 2001, Mamudu 2005). This compels the decision makers to depend on the experts for their knowledge-based information, technical advice, and expertise for solving the societal problem.

However, as the interest for information and interpretation persists, these experts who are bonded by their shared beliefs and reasoning as well as policy preferences become institutionalized and influential in the politics of the issue area (Haas 1992, Edwards 2001). It must be noted that the epistemic communities are not policy advocates in the real sense. Policy advocates are individuals who persistently lobby for certain proposals to be adopted as policy, while the epistemic communities are experts who share a common belief about a problem and the means of solving the problem. However, there may be some overlap between the activities of the two groups. Therefore, when the need arises for their expertise to be used to address a global problem, the experts capitalize on that opportunity to seek for global adoption of their preferences through policy diffusion. The activities and characteristics of epistemic community differ from policy advocates (See Figure 2.2). 


\section{Figure 2.2 Structures of Activities of Epistemic Community}

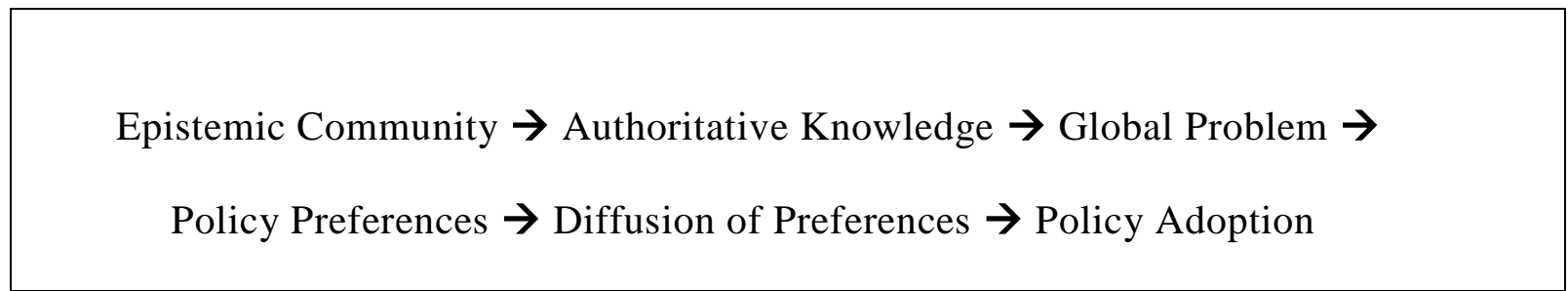

Source: Edwards 2001; Haas 1992

Edwards (2001:9) maintains that epistemic communities emerge because of their interest to influence policy making in the area of their expertise, arguing that the influence comes with a reputation among their peers. He maintains that the influence generates access to funding, which encourages the institutionalization of the epistemic communities by attracting more experts who share similar ideas.

Alder and Haas (1992:377) and Haas (1992) note that epistemic communities also exert their influence on countries by helping the countries to identify their national interest or pointing out important issue areas that will enable the countries to deduce their interests. Haas (1992) argues that the epistemic communities can help to create and maintain social institutions that control behavior in the international scene. He further argues that the epistemic communities normally establish a model of cooperation within a certain issue-area that may continue to operate even when the organized concentration of their power is not adequate to influence countries to change their actions and interests. Haas (1992:3) identifies the distinguishing characteristics that set the epistemic communities apart from other members as:

(1) a shared set of normative and principled beliefs, which provide a value-base for social action of community members; (2) shared causal beliefs, which are derived from their analysis of practices leading or contributing to a central set of problems in their domain, which then serve as the basis for elucidating the multiple linkages between possible policy actions and desired outcomes; (3) shared notions of validity 
- that is intersubjective, internally defined criteria for weighing and validating knowledge in the domain of their expertise; (4) a common policy enterprise - that is, a set of common practices associated with a set of problems to which their professional competence is directed, presumably out of conviction that human welfare will be enhanced as a consequence.

The basic motive underlying the activities of the epistemic communities is to use their practical beliefs and ideas to transform the interests and actions of countries. Therefore, they use the interactions, dissemination of information, and the exchange of authoritative knowledge and ideas to achieve this purpose during periods of uncertainty (Wendt 1992, Edwards 2001). However, the issue for which the experts have a shared belief and seek to address through public policy must be of international nature in a way that it generates some form of international cooperation among the experts. To Mamudu (2005:42), the principled, normative, and causal beliefs shared, the notions of validity, and the common policy enterprise are the factors that bond the members of the epistemic communities.

Mamudu (2005) also maintains that the scientific method of inquiry serves as the core beliefs of the members, and the shared beliefs and norms motivate a common understanding of problems, the causes of the problem, and how to solve the problem. To Alder (1992:104), the focus on epistemic communities and their mode of operation helps to understand the influence of shared principle norms, normative, scientific, and technical knowledge on international cooperation. Mamudu (2005) further notes that the epistemic communities' concept helps to understand how groups of experts are able to trans mit socially constructed ideas that develop as a shared knowledge among them to the international scene, which are promoted for countries to adopt. 
Haas (1992:27) argues that the epistemic communities serve as a medium through which new ideas diffuse from societies to governments and also from one country to the other. Haas (1989:378) examines the influence of the epistemic communities using the Mediterranean Action Plan (Med Plan), which was a regime for maritime pollution control in the Mediterranean Sea. He argues that regimes can transform and offer power to new groups, who can use it to change the interest and practice of countries. Haas (1992:380) maintains that this occurs when a group with a common perspective manages to acquire and sustain control over a substantial policy domain, making them stronger and forcing countries to conform to their interest. To Alder (1992:106), the success of the activities of epistemic communities is dependent on historical contingency created by the state of technology, distribution of power in the international scene, and domestic political and administrative structures.

In the politics of tobacco control, the epistemic communities have used their power to project the long time notion of the need to control tobacco due to its harmful effects into the global scene in the form of recommended policy solutions/measures to be adopted by countries. Mamudu (2005:44) argues that the communities have a common belief that tobacco consumption is harmful for human beings and is creating a major epidemic. He argues that the members of the tobacco epistemic community connect through the internet with GlobaLink. Also, they participate in world tobacco control conferences such as the WCTOH and WHO where they share their knowledge with the tobacco control community. He further argues that the best practices of tobacco control the epistemic communities are influencing countries to adopt are scientifically proven to be effective for controlling 
tobacco. Therefore, they recommend them as appropriate measures for the countries to adopt. However, the effectiveness of some of the measures has often been disputed.

Haas (1992:17) asserts that the epistemic communities have advantage and power in policy debates because the claim to knowledge is supported by a test of validity. However, the identification of what is considered the best practices of tobacco control is subject to the bias of the members of the community (Mintrom and Mossberger 2008, Bardach 1994). Nevertheless, Sebenius (1992:325) argues that even though the core interest of epistemic communities is fostering the adoption of their recommendations and interest, their success depends on how well they articulate the science-driven ideas to influence its adoption as policy (Larsen 2009). A preliminary review of literature supports the view that epistemic communities play an instrumental role in influencing the developing countries to adopt the best practices in tobacco control in conjunction with other governmental and nongovernmental actors. Therefore, the activities of the epistemic communities are examined as part of the overall global advocacy network in the tobacco knowledge transfer processes (Farquharson 2003).

Heclo (1978) clearly illustrates the existence of issue networks on various issues in the policy process. Petracca (1992:70), extending Heclo’s concept, defines an issue network as a network of communication among knowledgeable people in certain policy areas, and this includes government officials, business people, legislators, lobbyists, academicians, and journalists. Furthermore, Larsen (2009) argues that an issue network consists of a broader group of members who engage in systematic but limited interactions that sometimes involve conflicting views on what constitutes policy problems and their solutions. Nonetheless, a policy community network can consist of small members that are closely in contact and have a 
consensual notion of problem as well as its solution within a particular issue area. Certainly, the actors operating in the tobacco control regime are knowledgeable about the hazards associated with tobacco consumption and they operate in a network where communication of relevant information is channeled from one area to the other. Therefore, they could be classified as a network of actors on the tobacco issue.

The transnational nature of the tobacco issue generates various networks of actors who operate in a transnational environment to seek redress of issues of common interest. Mamudu (2005:44), and Keohane and Nye (1972: xii) define transnationalism as the process whereby governmental and nongovernmental actors move tangible and intangible items across the boundaries of countries. The items moved across states' borders in transnationalism include money, information, physical objects, people, and other tangible and intangible items (Mamudu 2005:44, Keohane and Nye 1972). Mamudu (2005), Keohane and Nye (1972) argue that governmental and nongovernmental actors are key players in the transactions. The tobacco control activities certainly involve the movement of tangible and intangible items across borders by governmental and nongovernmental groups with the common interest of preventing the harm associated with tobacco. But the process often involves some politics and counter activities of opposing groups - the tobacco industry network.

Farquharson (2003) identifies the existence of a pro-tobacco advocacy network that operates in a similar manner as the anti-tobacco network. Members of this transnational tobacco interests network is made of the tobacco companies, epistemic communities, domestic tobacco growers, state-owned tobacco companies and other affiliates. The transnational tobacco companies use the their affiliates to obstruct any attempt by their government to adopt stricter 
tobacco control laws by projecting such moves as denying sources of employment and income to tobacco workers. For many developing countries, the contribution of tobacco to their political economy motivates them not to adopt stricter policies because of its perceived detrimental effect to the wellbeing of their economy (World Bank 1999; WHO 2008; Cairney 2007). Furthermore, the tobacco companies sponsor research which concludes with findings in support of the notion that the adoption of such laws will negatively affect the economy of countries that have tobacco as a great contributor to their GDP (Mamudu et al 2008).

The release of such studies and findings make it difficult for the government to adopt direct policies to address problems associated with tobacco production because the farmers respond negatively to the attempt of government to adopt such measure. Instead, the governments, especially those in democratic countries, resort to adopting indirect policies to address some tobacco related problems as means of protecting their political capital. As a result, this study expects that countries that have tobacco as a major source of earnings will be unwilling to adopt measures that could decrease their source of revenue and also to prevent negative reaction from the tobacco farmers and interest groups. Instead they will adopt voluntary agreement and laws that may indirectly address some of the problems associated with tobacco because the adoption of such laws will not attract any reaction from the tobacco interest groups. In view of the relevance of the approaches analyzed above to the politics of tobacco control, aspects of the approaches are also treated under the global advocacy network approach.

\section{Liberal-Constructivist Approach}

The Liberal-Constructivist approach brings together the liberal international theories and the constructivist theories. It focuses on how social interactions shape the interests and behavior of countries in the global system. Liberal theorists view international relations as a system of 
interactions in which it is possible for mutual gains to be made through cooperative and peaceful international behavior, i.e., they reject the idea of a "zero-sum game." Constructivists believe that most of the building blocks of world politics - such as state sovereignty, national identity, legitimacy, ideologies, and political institutions - arise from social action i.e., they are "constructed" and therefore can be changed. If the rules and norms governing behavior can be "reconstructed," new patterns of international behavior may emerge. Liberal-Constructivism emphasizes ideas and norms and how they change over time.

Research under a liberal-constructivist approach focuses on communications across cultures, and seeks to explain the creation of norms (e.g. "human rights"), their diffusion among individuals and political actors, and their effects on the behavior of those actors. The approach emerged in response to the assumptions of neoliberals and neo-realists that the interests of countries are given and can easily be detected (Finnemore 1996, McNamara 1998, Goldstein and Keohane 1993). The liberal-constructivist approach focuses on identity and interest definition and its influence on the actors as well as how information influences the understanding and actions of people (Wendt 1992, Mamudu 2005, Keohane 1989, Gidden 1996, Cook 1996).

Liberal-Constructivist also examines the influence of cultural practices, social values, and norm of behavior on political life and rejects the assumption that interest determines behavior (Finnemore 1996). Wendt (1992) notes that liberals believe international institutions can transform the identity and interests of countries. He indicates his dissatisfaction with that notion, arguing that what is considered as an "institution" is itself socially constructed. To him, what explains the transformation of identity and interests are ideas, information, and knowledge, which through their intersubjective and socially developed meanings and expectations provide understanding of identity and interests. 
Finnemore (1996: 15) notes that the approach centers on how choices are shaped by past dialogue, social norms, culturally determined roles and rules. She argues that the approach stresses the significance of knowing how actors develop knowledge about actions that are considered important, indispensable, and appropriate. She asserts that the intersubjective understanding of certain behavior prescribes knowledge and guides actions. She further indicates that the constructivist theory helps to understand how culturally constructed beliefs determine the actions and choices of actors as well as their outcome. Goldstein and Keohane (1993) argue that the political arena is characterized by conditions of uncertainty about the interests of the political actors. Therefore, the emergence of new ideas guides certain actors to define their interests and actions. They argue that the ideas are the principled beliefs, worldviews, and causal beliefs that give directions or provide convincing ethical and moral inspiration for action during condition of uncertainty (also see McNamara 1998). McNamara (1998:5) maintains that the ideas emerge from the interactions and experiences of actors with other actors and their environment.

Mamudu (2005:30) states that the core concern of constructivism is how states construct their identity and form their interests in the international arena. He argues that constructivists investigate how states' actions and behavior are influenced by principles, conventions, and norms of behavior. Finnemore (1996) also argues that the interest of constructivist centers on how political life is impacted by the values of society and cultural practices. Nevertheless, these societal values and practices are socially constructed. Wendt (1992:398) argues that institutional roles assign identities to actors, which in turn influence the interests of the actors. Therefore, it is presumed that the membership of countries in certain international institutions such as the Commonwealth and the World Health Organization, as well as the ratification of the FCTC, 
come with certain roles and identities that require countries to conform to certain international norms. The requirement to conform to these socially constructed norms compels the countries to change their actions and interests.

This is largely because the anti-tobacco activists have been able to elevate their socially constructed ideas into a global prohibition regime ${ }^{13}$ in the form of FCTC (Nadelmann, 1990). Accordingly, countries are being pressured to conform to the norm expectations through the adoption of the FCTC tobacco control measures. The underlying factor compelling the countries to conform to global norms is their membership of international organizations and the inherent role to protect citizens from a global epidemic caused by the consumption of tobacco products. The mechanism used to ensure global conformity to the tobacco norm expectation is interactions and the spreading of socially constructed ideas on the best practices of tobacco control aimed at compelling countries to change their interests and actions to adopt domestic tobacco control policies.

This study presumes that Ghana and Malawi are involved in constant interactions with transnational actors and agents of the knowledge transfer such as the U.S, Canada, U.K, WHO, the Commonwealth, the American Cancer Society, the Bloomberg Foundation, Bill and Melinda Gates Foundation, and certain epistemic communities in the tobacco control regime. The core issues of discussion in the interactions are information and ideas on the tobacco control best practices that will help the countries to conform to the norms of the FCTC. However, the interactions with actors directly or indirectly through some form of sponsorship, is resulting in a change in the way the tobacco issue is understood by the people and policymakers of both countries (Makoka et al 2011).

\footnotetext{
${ }^{13}$ Refer to page 66 for the definition of prohibition regime
} 
In addition, the change in the understanding of the tobacco issue is generating a change in the actions and interests of the developing countries, which is motivating the countries to adopt the tobacco control ideas as domestic tobacco control laws. As a result, this study expects that the change in the understanding of the tobacco issue facilitated by the dissemination of new tobacco control ideas will encourage support for the adoption of domestic tobacco control laws. Therefore, the review of the politics inherent in the dissemination of the new tobacco control ideas will help to understand why the developing countries decide to adopt or not to adopt domestic tobacco control instruments.

\section{Liberal -Constructivism and Tobacco Prohibition Regime}

Evidence of the activities of the tobacco control actors show similarities with the prohibition regimes instituted to address the issues of prostitution, slavery, piracy, the killing of whales and elephants, extradition, and psychoactive substances, which were identified by Nadelmann (1990: 522-526). In spite of this, the notion that tobacco would likely be a target for an international prohibition regime because of the health hazards associated the consumption of tobacco products globally was initially downplayed (Nadelmann 1990; Studlar 2002).

Nadelmann (1990:484-5) maintains that the prohibition regimes normally witness four to five developmental stages. He argues that in the first stage, the activity being proscribed is normally considered legitimate by most societies under certain circumstances (also see Studlar 2002). He also indicates that states are mostly the central characters and accessories of the activities at this stage and bilateral treaties instead of moral or international norms are the mode of operation because limitations to the activities of actors are of prudence (Studlar 2002: 277). 
Nadelmann (1990:485) argues that international legal scholars, religious, and other moral entrepreneurs generally redefine an activity as a problem and an evil at the second stage. He maintains that even though individual governments continue to tolerate or even sponsor the involvement of private groups and individuals in the activity, the explicit government involvement in the activity is gradually delegitimized. During the third stage, proponent of the regime, especially those who could exercise hegemonic powers in certain issue areas and transnational moral entrepreneurs, actively agitate for the formation of international conventions and the suppression or criminalization of the activity by all states.

The agitation of the proponents takes several forms, ranging from diplomatic pressures, economic inducements, military interventions, and propaganda campaigns of the governments to the domestic and transnational lobbying, educational, organizational, and proselytizing efforts of individuals and nongovernmental organizations. He further argues that a fourth stage could emerge if the activities of the regime proponents are successful. In this case, the fourth stage witnesses the metamorphosing of the activity into subject of criminal laws and police actions globally. Subsequently, a global prohibition regime is established whereby international institutions and conventions emerge to play a coordinating role of the activity.

So far, it can be argued that the activities to control the harm associated with tobacco consumption has reached the fourth stage and a tobacco control prohibition regime is already in place. The FCTC under the WHO is the umbrella body coordinating the tobacco control activity and the FCTC has developed tobacco control measures that are being adopted into laws by countries to regulate the behavior and actions of actors affiliated with the tobacco activity. There is also a strong social pressure on all countries to adopt and enforce the FCTC measures, which is one of the core characteristics of the establishment of a prohibition regime (Nadelmann, 1990). 
In addition, the proponents of the FCTC are still dealing with deviant countries that have refused to sign and ratified the FCTC and weak countries that have ratified the protocol but are struggling to adopt and enforce effective tobacco control laws to end the hazards associated with tobacco consumption. Therefore, this study expects that the transnational tobacco control actors will mount some pressure on countries such as Ghana that has signed and ratified the FCTC but has not adopted effective laws to control tobacco policies, to do so. In addition, Malawi, which has refused to sign or ratify the FCTC protocol, will also experience the activities of the global tobacco control actors as means of getting her to conform to the global tobacco norm.

\section{Agents and Structure}

The issues of agency and structure have also been central to the analysis of the determinants of knowledge transfer. Finnemore (1996) argues that the core issue with understanding why certain countries adopt any policy has always been with examining whether social structures are presumed as emanating from the interaction of actors (agents), their preferences and capabilities, or considering if the choices and abilities of the agents result from the social structures that the actors are entrenched in. Ladi (2005) refers to agents as an entity capable of making decisions. She identifies structure as a dynamic phenomenon that changes overtime and notes that structure always constitutes an external environment to the actions of the agent. She cites examples of structure as organizational rules and imperatives, new knowledge, or ideas.

Ladi (2005) argues that countries could be considered as a structure and pressure groups as agents when the focus is on interactions and political activities within a country. On the other hand, when the international environment is the focus, states become the agents and 
globalization and Europeanization considered as the structure. Whereas some scholars focus on the activities of agents as the determinant of knowledge transfer, others concentrate on the influence of structure. Evans (2004) and Ladi (2005) argue that when the focus is on the agents, the concentration is normally on the micro level analysis, which entails the examination of the activities of the agents participating in the knowledge transfer. On the other hand, the macro level analysis focuses on the structure and the ideas embedded in the structure and how the ideas influence knowledge transfer.

Some scholars adopt the multi-level policy transfer approaches and examine the combined influence of the macro and micro actors on knowledge transfer. For instance, Dolowitz and Marsh $(1996,2000)$ focus on the micro and macro actors and examine how both voluntary and coercive policy transfers processes impact knowledge transfer. They also look at the causes and impact of policy transfer and how policy transfers lead to policy outcomes. Evans and Davies (1999) and Ladi (2005) add the intermediate or meso level approaches such as advocacy network theory to the micro and macro in the analysis of knowledge transfer (also see Common 2001). Evans and Davies (1999) argue that the interaction between the structure and agents occur at the intermediate level and that it is the interaction that generates policy transfer. They argue that change is mediated by the interactions, activities, and role of policy transfer networks. In addition, change can be influenced by the activities of states, global, international, and transnational actors and forces (Evans and Davies 1999, Ladi 2005, Common 2001). Scholars have also investigated the details of how policy makers acquire knowledge by examining the complex interactions between the domestic and the international policy actors as well as the interaction between state and non-state actors. 
The knowledge transfer and the global advocacy network approaches focus on analyzing how governmental and nongovernmental actors influence policy change. Therefore, the two theories fall under the agency approach. On the other hand, liberal - constructivist approach concentrate on the use of new tobacco control ideas to influence policy change and so it falls under the structural approach. Given that the tobacco control knowledge transfer is influenced by both domestic politics and international politics, the combination of these theories helps in the explanation of the process. Therefore, this study investigates the influence of agency and structure to determine how all the approaches collectively help in understanding knowledge transfer leading to policy change.

\section{Variables}

The dependent variables are the set of policies adopted by the developing countries. The independent variables are: (1) the composition and activities of the actors involved in the entire adoption process, pro and anti -tobacco advocacy networks such as tobacco companies, farmers, politicians, countries, IGOs, NGOs, epistemic communities and their impact; (2) the specific strategies and types of knowledge being transferred and the extent to which they include instrument(s) recommended by the FCTC such as the policies to protect nonsmokers from environmental tobacco smoke, labeling of cigarette packs, increases of taxes, etc, (3) the manner of transferring the knowledge (coalition or independently; official meetings, or correspondence through emails, private meetings, or the reading of literature); and (4) the factors that influence the adoption of a particular knowledge or tactics into domestic law in a specific country.

The other independent variables are: (5) the nature of the entire process/political struggle: opposition and support for adopting domestic laws, and internal and external pressure; (6) the 
impact of political economy on the adoption process; (7) the impact of IGOs on the process and the institutional venues such as the conferences, seminars, and workshops, etc, for the knowledge transfer; (8) the impact of signing and ratifying the FCTC protocol on the adoption of effective tobacco control legislation; (9) measures adopted by the knowledge transfer agents to enforce compliance with the FCTC norms (10) the changes in the understanding of the tobacco issues among the people and factors accounting for the changes, and (11) the impact of the bureaucratic agencies on the adoption of tobacco instruments.

\section{Research Questions}

The specific questions for the research are:

1. What is the nature of the tobacco control policy adoption process of Ghana and Malawi (the political struggles)?

2. What is the composition of actors involved in the tobacco control activities?

3. What is the specific tobacco control knowledge being transferred to Ghana and Malawi and the manner of transferring the knowledge?

4. Which factors influenced the adoption of a particular transferred knowledge or tactics as domestic tobacco control policies in Ghana and Malawi?

5. What impact does the domestic tobacco control actors and tobacco interest groups have on the adoption of tobacco control policies?

6. What impact does IGOs have on tobacco control knowledge transfer?

7. What manner of transferring tobacco control knowledge is more successful? In other words, is knowledge transfer successful when transferred through a coalition of actors or individually; or when it is coerced or voluntary?

8. How are the transnational tobacco control actors enforcing conformity with the norms of the FCTC?

9. Does the signing and ratification the FCTC protocol has any corresponding impact the adoption of effective domestic tobacco control law?

10. Do the domestic production/growing of tobacco or the presence of a tobacco manufacturing company affect the adoption of tobacco control laws? 
11. How is the control activity impacting the understanding of the tobacco issue and action of policy makers in the receiving countries?

12. Does the structure of bureaucracy have impact on the adoption of tobacco control instrument?

\section{Methodology and Data Collection}

This study relies on multiple methods of data collection to do its analysis and to draw conclusions (King, Keohane and Verba 1994). It adopts the most similar systems design to examine the similarities between the policy adoption processes of the countries (Manheim et al 2006; Peters 1998), which enables the variations among the processes to be detected.

Furthermore, it uses qualitative method of data analysis (George and Bennett 2005). It is a comparative case study that uses a triangulation approach involving both primary and secondary sources of information and data collection methods (George and Bennett 2005). The countries used for analysis, Malawi and Ghana, have similar but sometimes different patterns of policy adoption (Asare 2007).

The greatest component of data collection was through interviews of experts and officials from both the developed and the developing countries who are knowledgeable about the tobacco control policies and the inherent political struggles during the policy adoption process in the developing countries. In some instances, the materials and reports by the experts provided relevant information to complement the information obtained through the interviews. Interviews were used because it have been found to be a useful means of collecting data for both qualitative and quantitative research (Arksey and Knight 1999; Creswell 2003; Merriam 1998; Patton 2002; Rubin and Rubin 1995). The interviewees were mainly tobacco control experts and researchers, government officials, officials from NGOs and IGOs as well as individuals/activists selected 
from both the developed and developing countries. Many of the government officials work with the health department and enforce tobacco control voluntary agreement or represent the government at tobacco conferences. Therefore they are knowledgeable about the politics of adopting domestic tobacco control laws. In both countries the Ministry of Health and the officials have been responsible for issuing directives for voluntary agreements and other tobacco control activities.

The officials have firsthand information of tobacco control policies, and they educate the public about the dangers of smoking (Asare 2007). As a result, their views expressed in interviews and reports sent to donor agencies provide important information on the nature of struggles. Other groups who are equally well informed about the politics of tobacco control because of their involvement in the process are the NGOs, interest groups, and individual advocates of tobacco control. These organizations and individuals lobby the government to adopt tobacco control policies and also educate the public on the harmful effects of tobacco. For instance, NGOs like the American Cancer Society and the Bloomberg Foundation provide funds for undertaking extensive public education on the harmful effects of tobacco and also for lobbying the government for the adoption and enforcement of effective tobacco laws. In addition, individuals such as Mr. Kondwani Munthali - the coordinator of the Smoke Free Malawi - and Mr. Issah Ali - the executive director of VALD in Ghana - are actively involve with the tobacco control activities of the countries, therefore they are highly knowledgeable about the politics that characterize the process.

Some of the NGOs also undertake extensive research on the impact of tobacco and work with the Ministry of Health to promote tobacco control. There are also people who conduct research on tobacco and use the findings to put pressure on policy makers to adopt domestic 
tobacco laws. Some of the researchers work in academia, research institutions, and health institutions and have several publications on tobacco issues in their countries (Asare 2007). In all, 30 experts with adequate knowledge on the politics of tobacco control in the two countries were identified and 20 of them provided information for the study. The breakdown of the participants is as follows: Ghana-4, Malawi $-3^{14}$, Bloomberg Foundation -2 , America Cancer Society - 2, Campaign for Tobacco Free Kids - 1, Tobacco Control Researchers who have conducted research in the countries -4 , and WHO - 2, IDRC -2. Previous studies have shown that 20 -30 experts who are well versed in an area of study could be a good source of information for a study when their views are complemented or cross examined with existing secondary data (Mamudu and Studlar 2009; Asare 2007; 2009).

At least one of the participants from each country was actively involved in tobacco control either as an official working in the public health sector or in a relevant government ministry or engaged in tobacco control research. For instance, the information on Ghana came from individuals who are working in the Ghana Health Service, the Food and Drugs Board, views of members of the national steering committee presented in recently published research, researchers who have published extensively on tobacco control in Ghana, and members of NGOs actively involved in tobacco control. The Ghana Health Service is the main government agency responsible for implementing tobacco control policies in Ghana and the national steering committee is the official committee that has been tasked with the tobacco control advocacy and policy formulation. Some of the participants also had dual representation, working in the health department or academia and NGOs at the same time.

\footnotetext{
${ }^{14}$ Refer to the appendix for the details on the interview participants from Ghana and Malawi
} 
Efforts at obtaining participants from the Commonwealth Secretariat, the Ministry of Finance, Ministry of Agriculture, and the tobacco companies were not successful. The information on the Commonwealth was obtained through literature review and information from the website. In much the same way, the information on the economically related ministries and tobacco companies was also obtain from the review of literature and from researchers who have conducted similar research. Therefore, the information obtained from the interviews may not reflect their views and be bias in that sense. The detail of some of the participants is provided at the appendix page. However, some requested to remain anonymous for personal reasons and also in line with the introductory letter approved by the IRB, which indicated that the results of the research will be analyzed in aggregate without identifying individual participants (See a copy at the appendix page). Many of the interviewees were well informed about the policy culture of the adopting countries and the operations of the knowledge transfer agents.

\section{The Process of Interview and Data Analysis}

The interview participants comprising governmental and nongovernmental officials were selected through a purposeful and snowball sampling method. Merriam (1998: 61) argues that purposive sampling "is based on the assumption that the investigator wants to discover, understand, and gain insight of an issue and therefore s/he must select a sample from which the most can be learned" (also see Asare 2007). In addition, Berg (2004) and Asare (2007: 68) argue that through purposive sampling, researchers are able to apply their knowledge of the research problem to select experts who can help them to understand the issue being studied. The initial officials for interviews were identified through a review of the tobacco control literature and recommendations by tobacco control researchers. Afterwards, a purposeful sampling method was 
used to select the interviewees who were contacted. The first group contacted was asked to recommend other governmental or nongovernmental officials who are either directly involve or are knowledgeable about the processes of the tobacco control knowledge transfer of the countries. In some instances, the person contacted recommended others to be contacted for interviews or declined to participate. Some of the officials recommended were then contacted and interviewed. In general the interviews were conducted through telephone, face-to-face, and internet email correspondence.

The questions for interviews were formulated based on the review of existing literature. The questions were few and less structured to allow for follow up for in-depth understanding of the issues where necessary (Asare 2007, Creswell 2003). In addition, existing materials comprising reports and documents on the tobacco policies of the adopting countries and knowledge transfer related activities - conferences, seminars, workshops - as well as the periodic publications of the World Bank, WHO, the Commonwealth, and American Cancer Society on tobacco policies of countries were reviewed. Whereas most of this information was obtained directly from the adopting countries, others were obtained through the websites, media reports, and periodic reports/publications of the organizations. Other information was also obtained from the relevant agencies of U.S and Canadian governments, the NGOs, and epistemic communities.

The strategy helped to overcome problem of data collection resulting from the limited ability to obtain relevant information from the adopting countries. The study emulates the research strategies of Studlar (2002), Mamudu (2005, 2008) and Asare (2007) in which interviews of tobacco control experts and review of existing documents were used to draw conclusions. Cross checking with the interviewees from time to time were essential component of the study to ensure the authenticity of data and responses as well as the validity of inquiry 
(Merriam, 1998). The data collected through the responses from the interviews and review of the existing literature was analyzed qualitatively. In particular, the questions for the study were analyzed using the data collected from the interview responses and the existing documents provided by the interviewees. The study uses the information obtained from the responses to the interviews and existing documents to assess the research questions and also as the basis of drawing conclusions.

\section{Conclusion}

The study adopts an approach that combines three theories - multi-level policy transfer, global advocacy network, and liberal-constructivism - as a single framework to understand the process of policy change in Ghana and Malawi. The combination of the theories thrives on the assumption that several factors affect policy adoption and policy change in each of the countries. Moreover, the study finds these theories to be complementary for a detailed understanding of the process of policy change. It anticipates that there are political struggles that occur between the tobacco industry network and the anti-tobacco control network during the process of tobacco control policy adoption in each of the countries and combining these theories helps to get the big picture of the scenario. 


\section{Chapter Three}

\section{Nature of Tobacco Control Activities and Policy Change in the Africa Region}

\section{Introduction}

Since the 1990s when tobacco control became an international issue, almost all countries globally have had some activity on tobacco control in one form or the other (Mamudu 2008, Cairney et al 2012). Recently, there is virtually no developing country that does not have some program on tobacco control, in spite of the fact that developing countries lag behind the developed ones in the volume of tobacco control policies and programs (Cairney et al 2012; Mamudu \& Studlar 2009). Despite the active influence of tobacco companies and affiliates, and the similarities between the projected tobacco epidemic between the two worlds, a different pattern of the policy process has emerged for the developing countries and the developed countries partly due to the active role of international organizations and transnational tobacco control actors (Cairney et al 2012; Mamudu 2008; Mamudu \& Glantz 2009).

The tobacco control knowledge transfer agents often use a regional approach to transfer knowledge to present a uniform strategy for controlling tobacco because of the similarities in the operations of the tobacco industry in the developing countries. In addition, the use of the regional approach ensures that the meager resources could be used to transfer the essential tobacco control information to as many countries as possible in Africa (Interview 2010). This chapter offers a broad overview of the knowledge transfer process for the Africa region and examines how the network actors in tobacco influence policy change in certain jurisdictions. Specifically, the chapter examines the goals, influences, nature of operations, and strategies adopted by the actors in the trans-border advocacy network to influence the behavior and interests of their target actors. It investigates how the actors of the network influence countries to change their interests 
and behavior to adopt the preferred policies and actions of the network actors. The chapter also examines the notion of policy change focusing on the various scenarios under which change occurs. Moreover, it draws on the broader meaning of policy change to establish the expectation of policy change in tobacco control. It further investigates how agent and structure affect policy change. It also examines in detail, the various instruments of the FCTC and uses them as guidelines for the anticipated policy change influenced by network actors in the receiving countries.

The questions used in this chapter to examine how the trans-border advocacy network approach impacts tobacco control policy change in the African region include: (1) who is the actors involved in the trans-border advocacy networks? (2) What are the motivations of the transborder advocacy network actors and how do they achieve their objectives? (3) What are the strategies used by the advocacy networks to influence their target actors? (4) What are the main networks in tobacco regime and their activities? (5) How does the transnational advocacy networks impact policy change (6) how can the FCTC measures influence policy change and what role do knowledge transfer actors play in the process? (7) What is the strategy used by the transnational tobacco control actors to effect policy change in the African region (8) How is the tobacco prohibition regime being used to effect policy change in the developing countries? The chapter concludes with an examination of anticipated nature of policy change in the tobacco control policy subsystem in the developing countries.

\section{Actors in Trans-border Advocacy Network}

Since the period that scholars like Raymond, Aron (1966) introduced the concept of "transnational society"- to describe the interconnectedness and interchanges among actors in the 
international arena - into the international relations literature, several studies have emerged on this topic in all disciplines. In the international relations literature itself, the concept influenced the thinking on the activities of actors in the international system, and generated subsequent interest in this area. The shift in the perception has brought a lot of studies that focus on the activities of actors - interactions and cooperation among governmental and nongovernmental actors - across the boundaries of countries.

Some of the studies have re-examined the state-centric focus of international politics and given recognition to the vital role played by non-state actors in international affairs (Kaiser 1971, Risse-Kappen 1995, Keohane and Nye 1972). Others have analyzed the structure of actors in the transnational network and used various theories to explain or justify the reasons for the emergence of transnational activism involving both state and non-state actors. Nye and Keohane (1971:733) refer to activities of the actors that transcend the borders of countries as transnational relations, noting that it involve interactions between governments, international organizations, nongovernmental organizations, and individuals. These governmental and nongovernmental actors are considered as major actors of world politics because of the vital resources they control in specific issue areas (Piper and Uhlin 2004; Keohane and Nye 1972). Nye and Keohane (1972) maintain that the actions of these actors generate major changes across the boundaries of individual countries. Preliminary review of the activities of the tobacco control shows that there is a collaboration of nongovernmental and governmental actors who are operating across the borders of individual countries to impact policies changes on the tobacco issue.

The organizations, governments, and individuals collaborate to pursue issues of common interest across the borders of individual countries in a way that they influence the 
behavior and activities of other actors who may not share the interest uniting the group. Although the actors in the network are generally bonded by common interest, some members may differ on the means through which such interest could be addressed (Diani 2000). While some consider certain issues as local or national, others may see them as global, yet they collectively operate to address the issue. For instance, some activists of tobacco control consider the tobacco issue as local and therefore seek local, culturally sensitive policies to address the issue. Others however, consider the issue as global and push for the adoption of globally scientific policies for addressing the issue. Even when the tobacco issue is seen as local, the policy outcome could be influenced by the interplay of domestic societal forces with the transnational dynamics because of the nature of the tobacco issue and the activities of the transnational tobacco companies.

The transnational actors who have been diffusing tobacco control measures to the African countries mostly focused on protecting the interest of the voiceless and the weak in international tobacco control politics by assisting the victims to resist activities considered detrimental to the wellbeing or the achievement of their rights as human beings. The conviction of these governmental and nongovernmental actors motivates them to assist local organizations and policymakers with funding, technical assistance and skills for adopting appropriate domestic tobacco laws. Moreover, these organizations and actors act as channels through which tobacco control policies diffuse to the developing countries, which enables the developing countries to partake in the administration of global tobacco control (Cairney et al 2012).

Alternatively, members of the tobacco industry network actors are bonded by the desire to promote the interest of the tobacco industry and activities linked to the industry 
(Farquharson 2003). The actors connected to the tobacco industry adopt strategies across the borders of countries to resist the activities of tobacco control activists who are seeking to end their business globally (Farquharson 2003; Cairney et al 2012; BAT 1980). The actors in the tobacco industry network comprise transnational tobacco companies such as BAT and Phillip Morris, domestic tobacco manufacturing and leaf buying companies, international and local tobacco growers associations, farmers, businesses, distributors, researchers, individuals, advertising, and hospitality industries (Farquharson 2003; Cairney et al 2012; INFOTAB \& Advisory Group 1983; B. Simpson \& INFOTAB 1985; Verkerk \& INFOTAB 1985).

The extent of wealth of the tobacco industry enables the tobacco network to support its members to organize and adopt effective resistance measures against the adoption of policies that will hamper their successful operation. Nonetheless, the resources also enable the industry to use monetary and employment incentives to win the support of influential policy makers or to penetrate and dilute the operating machinery of tobacco control activists (WHO 2008). The actions sometimes forestall the ability of tobacco control activists to push for the adoption of domestic tobacco laws that are unfavorable to the tobacco industry. The wealth also enables the tobacco companies to hire people to conduct research and present reports favorable to the interest of the industry and also to employ the media propaganda to protect their interest (Mamudu et al 2009). In addition, the network is able to alert members of unfavorable policies adopted somewhere and prepare their members to prevent the adoption of similar policies in their areas. Generally, the role performed by the network across the developing countries includes undermining tobacco control programs and 
policies, expanding and consolidating new markets, as well as promoting the use of tobacco products (Cairney et al 2012).

\section{Motivations and Objectives of the Network Actors}

According to Keck and Sikkink (1998), transnational activities involving civil society organization is not a new phenomenon. They maintain that actors who are interested in issues that extend across the borders of different countries have been involved in such activities since the nineteenth century or earlier, during the anti-slavery movements. Trubek et al (2000), note that the motivating factor underlying transnational activism is the belief among actors that different actors can mobilize norms in different arenas to exert pressure on their targeted actors elsewhere. To that effect, transnational actors have exerted substantial influences on the activities of countries and shape their behavior on many issues (Cairney et al 2012; Sikkink 1998; Stone 2000). The underling factor for the emergence of transnational/international relations is the increased interdependence, improvement in communication technology, and the desire to cooperate to achieve mutual interest among governmental and nongovernmental actors across states' boundaries (Nye and Keohane 1971). Moreover, the penetration of the barriers between individuals, states, and organizations across the boundaries of countries through the "telecommunication revolution" has made it possible for likeminded actors to share ideas and experiences around the world (Slaughter 2004).

Piper and Uhlin (2004:3) maintain that one of the ways that transnational networks are created is when nongovernmental organizations and social movement actors undertake activities that extend beyond boundaries of individual countries. Moreover, the movement of tangible and intangible items across state boundaries by actors, one of which may be a 
nongovernmental actor also breeds transnationalism (Keohane and Nye 1972). The items moved across states' borders by the actors include money, information, physical objects, people, and other tangible and intangible items (Keohane and Nye 1972; Mamudu 2005; 2008). However, conflict can also generate transnational activism when nongovernmental actors form alliances with state actors in response to conflict with corporations and other state actors (Piper and Uhlin 2004).

In the area of tobacco control, some studies have examined the alliances between nongovernmental and governmental tobacco control activists to confront the activities of the tobacco industry network (Mamudu 2005; Mamudu and Studlar 2009; Mamudu and Glantz 2009; Faquharson 2003). For instance, Mamudu (2005, 2008) uses the liberal-constructivist theory to analyze transnational activism in tobacco control. He focused on the factors and interactions that enabled a global tobacco control regime to emerge under the auspices of the World Health Organization Framework Convention on Tobacco Control (WHO-FCTC). The World Health Organization (2008) maintains that the tobacco control organizations have been rallied by the concerned about health hazards of tobacco consumption and the rapid relocation of the tobacco companies from the developed to developing countries.

Hudson (2001) argues that the linkage between the network actors in the south and north enable them to influence policies and actions of individual consumers, countries, companies, regional, and international institutions. Even though the specifics of how to prevent the health hazards associated with the consumption of tobacco may differ among the network members, there is a general agreement on the need to control the consumption of tobacco and its health hazards among the transnational advocacy network actors. Simultaneously, the activities of the tobacco control interest groups and actors to halt the activities of the tobacco industry network 
have attracted counter measures from actors linked to the tobacco industry, who have mobilized to protect their interests and businesses ${ }^{15}$ (Cairney et al 2012; BAT 1980). A clear observation is that the actors in the different networks in the tobacco control regime are connected by different reasons and interests, but the strategies each network adopts to achieve it core objectives are similar.

\section{Strategies, Power Structure, and Operations of Actors}

The power structure of the global advocacy network is not clearly defined because the network members are amalgamation of activists loosely connected by issue of common interest and shared beliefs. Jordan and Tuijl (2000:2053) and Keck and Sikkink (1998) define activists as people who are prepared to incur considerable cost and act to achieve goals they care about deeply. The core instrument in a global advocacy network is the use of persuasion to influence decision making and behavioral changes in multiple political arenas. In that sense, the activists' core responsibilities are targeting a specific arena at either the local, country, regional or international levels. However, there is no formal mechanism for enforcing the obligations of the activists within the networks (Jordan and Tuij1 2000). This makes it difficult to have a group that has total control over the activities of others within the network, and the best phrase that can be used to describe the nature of power within the network is a fluid structure of power distribution.

One strategy the advocacy networks use to confront the status quo is global campaigns that focus on the dissemination of relevant information and transfer of expertise (Keck and Sikkink 1998, 1999, Jordan and Tuijl 2000, Trubek et al 2000). Additionally, the actors in the global advocacy network engage in research and the analysis of policies and activities of

\footnotetext{
${ }^{15}$ Please refer to the section on the actors in trans-border advocacy network for details of transnational activities of the tobacco industry to protect its interest.
} 
corporations and states, which enable them to channel relevant information to influence the actions of their target actors. Hudson (2001) argues that in addition to campaigning, the actors use awareness-raising, development, education, lobbying, capacity building, and occasionally, direct action as tools for achieving their objectives. Keck and Sikkink (1998:89) note that transnational advocacy enables the network actors to strategically mobilize relevant information, which are used to "persuade, pressurize, and gain leverage over more powerful organizations and governments". They maintain that the actors are able to change the structure of policy debate and impact the outcome of policy through such efforts. Trubek et al (2000) argue that the transnational actors are motivated by the belief that they can marshal and produce norms in different arenas, which then can be used to exert pressure on their target actors and also to achieve their objectives in different political arenas. The actors are convinced that the ability to act in unison with regard to the adoption of tobacco control policies in each country through their tobacco control efforts in different countries will leave no place for the tobacco companies to hide.

The ability to frame issues and disseminate information in support of their frames is central to activities of the global advocacy networks. Keck and Sikkink (1999: 90) maintain that the actors are able to gain an audience and attract attention by framing their issue to match the correct institutional venue (also see Cairney 2007). Framing is "the conscious strategic effort by groups of people to fashion shared understanding of the world and themselves that legitimate and motivate collective action" (Keck and Sikkink 1999: 90, McAdam et al 1996:6). Keck and Sikkink (1999) maintain that the network actors use the control of relevant information and ideas to impact the structure and context of policies of countries. They also argue that the actors 
influence the public agenda by creatively framing issues, reframing old problems, and seeking hospitable venues to change the way the target actors understand their interests.

Keck and Sikkink (1998) identify the strategies used by the network actors to influence the behavior of their target actors as information politics, symbolic politics, leverage politics, and accountability politics. They maintain that in information politics, network actors are able to quickly and creditably transmit politically usable information to venues where it will have the most impact. Symbolic politics occurs when the actors use symbols, actions and stories to present problems of actors who may be far away so as to draw attention to their issue. In leverage politics, the network actors use powerful actors to champion issues in areas where weaker actors may lack the needed influence. Lastly, they maintain that the actors through accountability politics compel more powerful actors to fulfill principles and agreements they have previously endorsed.

Studlar (2002) argues that tobacco advocates in the U.S and Canada made a lot of impact in their campaign by framing the tobacco issues as the protecting the right of the non-smokers in the 1990s. He maintains that the framing of the tobacco issue as second hand or passive smoking changed the focus of the tobacco control campaign from protecting the rights of the smoker to that of protecting the right of the third party who has not consented to consume tobacco but are affected by the actions of the smokers. Asare (2007) makes a similar assertion, arguing that the issue of passive smoking brought to bear the need to protect the rights of the innocent child who is forced to inhale the tobacco toxic and its related health hazards because of the unhealthy choices of adults around the child. He argues that the framing of the issue as passive smoking gave impetus to the tobacco control campaign. 


\section{Advocacy Networks and Prohibition Regime in Tobacco Control}

Trubek et al (2000:1194) define "a regime as a set of institutions in international space that establishes and reinforces norms or rules of the game for public and private actors". They further argue that the regime theory alone does not help to adequately explain the transnational activities because the theory primarily focuses on structure and rules. On the other hand, the transnational activism centers on the activities of actors and agents. The global advocacy network theory analyzes the impact of structure, rules, agents and actors on the actions of target actors and helps to understand the impact of transnational activities. Besides, the networks are able to adopt norms from different sources to achieve the objectives of a regime (Trubek et al 2000).

Trubek et al (2000:1194) further argue that the actors are able to "mount campaigns that use international, supranational, regional, national, and local laws as well as private norms and political actions to affect the outcome in one or more countries". The core assumption underlying the mounting of campaigns is the belief that effective regulatory systems can be created through the mobilization of overlapping jurisdictions and the continued operation of network actors across the borders of individual countries. From the analysis above, a tobacco control regime can be described as the set of institutions that have emerged globally to reinforce the adoption of the WHO-FCTC measures as appropriate tobacco control laws at the domestic level. The tobacco control actors use information dissemination and campaigns, which highlights the FCTC measures as important international norm, as means of achieving their objectives.

The transnational actors promote the adoption of the FCTC measures as domestic tobacco control laws to proscribe the consumption of tobacco globally. The international tobacco control actors - especially from the developed countries - are putting social pressure on all countries to 
acknowledge and enforce the norms of the FCTC through their activities. The FCTC, now a well-established the tobacco prohibition regime, serves as the umbrella body that coordinates the transnational activities of the tobacco control actors (Nadelmann 1990; Studlar 2002). The objective of the regime actors who are promoting the adoption of the FCTC measures is to make the measures a subject of criminal law that will help to restrict the consumption of tobacco globally. The transnational actors are engaging the developing countries because many of the countries are weak and are unable to or do not have the political will to crack down on the violators of the international global norms. In addition, the transnational actors contact deviant countries such as Malawi and Indonesia who have refused to sign and ratify the FCTC protocol because of the dependence of their economies on tobacco to offer incentives that will influence the countries to diversify their economies.

The belief is that once the countries obtain other sources of economic activities and employment that contribute to their GDP, they will be willing to become parties to the FCTC protocol and also control tobacco. However, it needs to be noted that the transnational tobacco actors are not only the actors seeking to proscribe the consumption of tobacco. There are other actors who are preventing the adoption of effective domestic tobacco control laws. Generally, the activities of each of the actors clearly place them in one or the other networks within the tobacco control policy subsystem. Nevertheless, there are others who are not directly involve in a clear cut network but can only be linked to any of the networks through a thorough analysis of their activities and actions. 


\section{Pro-Tobacco Advocacy Network}

Farquharson (2003:82) argues that the pro-tobacco advocacy network (or the tobacco industry network) is made up of actors who are linked to the values and goals of the tobacco industry. The actors in the network include transnational tobacco companies (TTCs) that are very competitive, well- resourced and locally independent actors as well as the associated trade organizations (Farquharson 2003). The activities of the network in the developing countries started with the TTCs and manufacturers associations in the 1980s but later extended to incorporate interested actors from farmers/leaf growers, dealers, and advertising and hospitality industries (INFOTAB \& Advisory Group 1983; B.Simpson \& INFOTAB 1985; Verkerk \& INFOTAB 1985; Cairney et al 2012). The members are connected by a common interest built around the protection and sustenance of the tobacco industry.

The pro-tobacco network is very effective in the dissemination of strategic information to their target audience but is very secretive about its advocacy activities (Farquharson 2003). The threat of reports that linked tobacco smoking to lung cancer by the Royal College of Physicians and the United States Department of Health Education and Welfare in the 1960s has united the tobacco companies to protect their common interest and increased their scope of operation (BAT 1980). The tobacco industry network has systematically monitored the tobacco control policies of individual jurisdictions and used their research and public relations strategies to avoid the adoption of stricter regulatory policies that will hamper their activities (Studlar 2002; Asare 2007; Mamudu and Glantz 2009; Farquharson 2003).

The resources enable the network to penetrate the developing countries in a top-down manner to influence their policy behavior (Cairney et al 2012; Chitanondh 2001, The U.S. Department of Health and Human Services 1992). Once in the developing countries, the TTCs 
encourage the use of tobacco products with advertising, promotional, and marketing strategies that are highly sophisticated (White 1997; Cairney et al 2012). The resources also make the TTCs attractive to countries that are seeking developmental projects by allowing the companies to establish new manufacturing plants, purchase diversified state companies, or engages in joint ventures ownership (Cairney et al 2012; Aguinaga \& Shatenstein 2002; Collin et al 2004; Gilmore \& McKee 2004; LeGresley et al. 2008; O’Sullivan \& Chapman 2000; Shafey et al 2009; WHO-EMRO 2004). Apart from the legitimate attraction, the TTCs also maintain their presence in the developing countries through illicit trade, which makes their products available to the domestic markets to undermine tobacco control activities (Shafey et al 2009; Guevara 2008; International Consortium of Investigative Journalists 2001; WHO-EMRO 2004, Cairney et al 2012).

Mamudu (2005) and Farquharson (2003) maintain that the International Tobacco Information Center, or INFOTAB, established in 1979, was the organization that connects the actors of the transnational tobacco industry network and also championed their cause during the 1980s. Farquharson (2003:85) argues that the International Committee on Smoking Issues (ICOSI) was a predecessor of the INFOTAB and it laid the foundation for the transnational tobacco industry network. The ICOSI was established in 1977 by the major tobacco companies to shape public opinion on tobacco issues through the media, conduct pro-tobacco research with sponsorship from the tobacco companies, and also to refute the claim that smoking has health hazards (Farquharson 2003; tobacco.org e-news 2001).

Like the ICOSI, the INFOTAB had the same objective but expanded its activities to include the "sharing of pro-tobacco data and the coordination of data and information in economic, scientific and technical areas, dissemination of pro-tobacco information in order to influence 
policymaking in the tobacco subsystem throughout the world" (Farquharson 2003:85; INFOTAB 1985). The INFOTAB established a project in the 1980s that enabled the tobacco interest groups and companies to monitor the tobacco control activities of the WHO and some nongovernmental organizations in the developing countries (Cairney et al 2012). The project also enabled the tobacco industry network to monitor the activities of the World Bank, IMF, and the UN Development Program related to tobacco control. This helped to undermine the tobacco control efforts by assisting manufacturers' association to politically resist the adoption of tobacco control measures and to organize workshop and promotional activities for refuting the negative information about tobacco consumption (Philip Morris 1981; Hargrove \& INFOTAB 1981; INFOTAB 1984; B.Simpson \& INFOTAB 1985).

Farquharson (2003) notes that the members of INFOTAB were both from the major tobacco companies and the representatives of the various manufacturers associations and INFOTAB provided them with the necessary tobacco information by analyzing the media coverage of tobacco issues (see also INFOTAB 1985). The INFOTAB enabled the tobacco industry network to keep track of developments within the tobacco industry globally on issue-byissue and country-by-country basis (INFOTAB 1987; Farquharson 2003). In addition, INFOTAB monitored the tobacco control policy developments, and provided a comprehensive report on anti-smoking conferences and policies as well as the plans to implement the objectives of the tobacco industry.

The pro-tobacco network is still in operation through informal organizations like the Industry Ingredients Group (IIG) in spite of the demise of the INFOTAB in 1991 (Farquharson 2003). The network continues to use the issue of political economy that stresses the importance of tobacco manufacturing and leaf growing to the GDP of the developing countries (Philip 
Morris 1981; INFOTAB 1990; Hargrove \& INFOTAB 1981; INFOTAB 1984; B. Simpson \& INFOTAB 1985, Cairney 2007). The network currently adopts a multilevel approach, which includes concentrating its efforts on particular tobacco control issues like second hand smoking and strategizing against the adoption of stricter regulation against tobacco products in any place to avoid its spillover effects in other areas (Farquharson 2003; Opukah 1996; BAT 1997; Cairney 2007; Cairney et al 2012).

The tobacco industry organized advocate groups such as the International Tobacco Growers Association (ITGA), an agricultural lobby group which champions the lobbying activities of the tobacco growing in third world countries at the World Health Organization (Yach and Bettcher 2000; Farquharson 2003). Furthermore, the tobacco industry network uses other strategies such as the corporate social responsibility through which developmental projects are handed over to local communities, employing deceptive regulatory codes, and making the claim that tobacco control is an issue of the developed world to undermine the tobacco control efforts (Cairney et al 2012). In other instances, the TTCs sue to overturn laws promulgated to control tobacco and also recommend weak tobacco legislation that will have minimal impact on their activities (Global Smoke-free Partnership 2009; Hirschhorn 2006).

Farquharson (2003) maintains that the tobacco companies sponsor domestic interest groups such as restaurants associations, domestic tobacco growers, and smokers' rights groups to champion its activities and obstruct domestic policy adoption (Samuels and Grantz 1991; Traynor, et al 1993; Ellis, Hobart and Reed 1996). For instance, companies such as BAT have used local tobacco farmers and businesses to lobby against the adoption of stricter tobacco policies in countries where the company operates (WHO 2009; Interview 2010). The activities of the tobacco industry are some of the motivating factors for this research as it seeks to examine 
and explain the different strategies used by the tobacco industry network to obstruct the adoption of relevant domestic tobacco laws in the developing countries.

\section{Anti-Tobacco Advocacy Network}

The anti-tobacco advocacy network is made up of an amalgamation of governmental and nongovernmental actors bonded by their commitment to halt the smoking addiction and health hazards being spread by the tobacco industry (Farquharson 2003; Mamudu 2005, 2008;

Nathanson 1999). According to Mamudu (2005, 2008), the activities of the anti-tobacco advocacy groups globally were energized by the official recognition of the harmful effects of tobacco acknowledged in the reports of the Royal College of Physicians and that of the U.S. Surgeon General in the 1960s. The two reports were followed by other research providing evidence of the strategy of the tobacco companies to spread the harmful effects of smoking through the expansion of their trade globally. This gave birth to transnational activities to control tobacco consumption through collaboration and sharing of relevant information. Mamudu (2005, 2008) maintains that following the reports, deliberate attempts were adopted by certain tobacco control interest groups, mostly from the developed countries, to organize advocacy groups to undertake tobacco control campaigns.

The advocacy network operates as an international tobacco control network (ITCN) to counter the trading activities and the influence of the tobacco industry network on policy making, especially in the developing countries (Cairney et al 2012). Moreover, the members of the network communicate through conferences and the internet to spread knowledge on the hazards of tobacco and strategies to influence policymakers to adopt tobacco control laws. The members also use legislative testimonies, legal suits, and campaigns to influence tobacco control 
policy outcomes. Mamudu $(2005,2008)$ further argues that the interest groups provide funding to other groups from developing countries to participate in the tobacco control conferences and meetings.

The conferences and funding have been the means of expanding the scope of advocacy against the control of tobacco consumption and the activities of the tobacco industry. The advocacy, which emerged under the umbrella of International Union against Cancer (UICC) and the International Liaison Committee on Smoking Control (ILCSC) in the 1970s and 1980s (Leonard Zahn \& Associates 1985), has in recent time come under the leadership of the American Cancer Society (The Observer 1985). Cairney et al (2012) maintain that the network has continuously championed tobacco control in developing countries in a bottom-up approach through the use of public awareness on tobacco consumption and its side effects and also by influencing policy development as well as mobilizing domestic forces to counter the activities of the tobacco industry network.

The 1990s saw an additional momentum to the anti-tobacco advocacy globally as more advocacy groups emerged to protect the interest of the innocent victims of tobacco, especially children, because of the availability of more evidence revealing the health hazards of second hand smoking on the nonsmokers (Nathanson 1999; Studlar 2002; Asare 2007; 2009; CDC 1999; Mamudu 2005; 2008). Alliances and coalitions of NGOs, specialized networks, and regional networks became part of the international tobacco control network (Cairney et al 2012; Philip Morris 1993). Cairney et al (2012) and Mamudu and Glantz (2009) argue that, organizations like the International Nongovernmental Coalition against Tobacco (INGCAT), International Network of Women against Tobacco (INWAT), and the Asia Pacific Association for Control of Tobacco (APACT) came under the Framework Convention Alliance (FCA) and actively influenced the 
development of the FCTC. The Framework Convention Alliance, which started as alliance of nonprofit organizations is now a nonprofit organization incorporated in the Switzerland with representative offices in Washington, DC and other places around the world. The FCA is currently made up of over 350 organizations from more than 100 countries working on the development, ratification and implementation of the FCTC international treaty.

Western philanthropists like the Mayor Michael Bloomberg of New York City and Bill and Melinda Gates have joined the ITCN to implement the FCTC measures in the developing countries through the provision of funding. A deputy director of the tobacco free initiative (TFI) indicated in an interview that the Bloomberg Foundation, and the Bill and the Melinda Gates Foundation are two major financiers of the activities of the FCTC in developing countries. He indicated that the WHO-TFI is umbrella body of the FCTC that oversees the implementation of the FCTC protocol. In addition, organizations and groups that were already in existence have incorporated tobacco control as part of their activities (Mamudu 2005, 2008). Farquharson (2003) maintains that the membership of the anti-tobacco network comprises: the international and domestic NGOs, intergovernmental organizations, governmental officials, individual researchers, and media practitioners. She argues that the activities of the anti-tobacco network are well coordinated and funded even though it is not as well funded as the pro-tobacco network (also see Mamudu 2005, 2008). She further observes that whereas the aim of the pro-tobacco network is to prevent the adoption of any form of tobacco policy that has the potential of hindering their activities, the anti-tobacco network actors focus on the promotion of a specific area of tobacco control. The members of the anti-tobacco network mostly champion specific types of tobacco control instrument(s) that interests them because of their belief in the 
effectiveness of those instruments in addressing the tobacco issue, in spite of the fact that the members are bonded by their core values and the interest to halt the hazards of tobacco globally.

The core values motivate them to share ideas and galvanize support for their activities through informal and formal means or to become members of a tobacco control epistemic community. The formal means of contacts are made through venues like GlobaLink (a web based forum) or conferences such as the World Conference of Tobacco or Health (Farquharson 2003, Mamudu 2005), and the meetings of the Commonwealth member countries. However, members who are not part of the GlobaLink communicate through other informal means to share ideas on innovative ways to control tobacco consumption. Other means of contacts are through joint research projects, invitation to serve on advisory group, or on expert panels (Farquharson 2003). Mamudu (2005: 79) argues that the members believe in collaborative actions involving individuals, communities, countries, regional, and international organizations as the effective means to control the spread of tobacco use and its health hazards globally. The intention of the collaboration is to adopt innovative tobacco control programs through the sharing of relevant information to combat the spread of tobacco health hazards and also to halt the activities of the tobacco industry.

In a nutshell, the core interest of the anti-tobacco advocacy group is to develop new and innovative ways to counter the activities of the tobacco industry and halt the hazards associated with smoking. Therefore, they use all the means available to them to transmit relevant information and strategies that will enable them to curtail smoking and its related harm. In that regard, this study examines the strategies employed by the anti-tobacco network to counter the activities of the tobacco industry through the promotion of the adoption of control laws in the selected countries. 


\section{Trans-border Advocacy Network and Policy Change}

The studies that address the determinants of policy change abound (Capano and Howlett, 2009; Rose 1991; 2005; Bennett 1991; Dolowitz and Marsh 1996; 2000; Bennett and Howlett 1992; Studlar 2002; Asare 2007; 2009; Evans and Davies 1999; Ladi 2005; Common 2001; Evans 2004; Stone 2000; Bomberg and Peterson 2000; Finnemore 1996; McNamara 1998; Wolman 1992). Generally, these studies assess the determinants of policy change through the lenses of policy transfer and focus on the role of policy transfer agent, discourse or structure (Ladi 2005). Studies focusing on the activities of agents normally concentrate on the action taken by actors like policymakers (Evans 1999; Rose 1991; 2005; Bennett 1991), international organizations (Bomberg and Peterson 2000), nongovernmental organizations (Stone 2000; Ladi 2005), or the joint action of governmental and nongovernmental actors (Keck and Sikkink 1998; Faquharson 2003; Studlar 2003) to facilitate policy change. Normally, the scholars analyze the actions of policy transfer actors by examining the will to voluntarily adopt certain policies or to exert some pressure on others to adopt preferred policies.

On the other hand, some scholars emphasize structure through which the agency obtains meaning in policy transfer. Scholars focusing on structure stress the impact of the external environment on the actions of agents and concentrate on the roles of ideas, organizational rules and imperatives embedded in the structure that facilitate policy change (Ladi 2005; Finnemore 1996; McNamara 1998; Hall 1993; Common 2001; Evans 2004). Therefore, their scope of analysis centers on the factors that influence policymakers and societies to adopt certain policy initiatives. Moreover, scholars focusing on structure tend to emphasize macro level activities while those that stress the role of agent in policy change concentrate on the micro. 
There are also scholars who focus on the interplay between structure and agents in policy transfer processes (Ladi 2005; Dolowitz and Marsh 1996; 2000; Stone 2000). Yet other scholars concentrate on the interactions between the structure and agents as means of understanding policy outcomes (Evans and Davies 1999; Common 2001). Such scholars comprehensively combine several approaches to examine the factors that influence policy change. Even though they use multi-level approaches to understand policy change, their analysis tend to treat ideas that facilitate policy change as given within a certain structure such as globalization or Europeanization. Nevertheless, liberal-constructivists who investigate how ideas are constructed within the structure, tend to focus only on that aspect in their analysis of policy change.

The trans-border advocacy network approach investigates policy change by combining all these approaches and perspectives. It operates on the assumption that what causes a particular jurisdiction or country to adopt a certain policy is a combination of factors, which includes the use of ideas that have been socially constructed by a group and are elevated as the core belief and practices within a certain structure (See Figure 3.1). Consequently, governmental and nongovernmental actors collaborate to spread their ideas to others for them to adopt as domestic laws. Therefore, the adoption of the idea or tactics into domestic law is influenced by the actions of policy transfer agents and system of new ideas that are socially constructed and are manipulated into globally accepted and binding requirements, and these are transmitted to create policy change in certain countries. In the tobacco control world, a group of actors who believe in the control of tobacco to avoid its harmful effects on health has been able to elevate their belief into a prohibition regime (Nadelmann, 1990) in the form of the FCTC, which has become a structure. These actors continue to promote their interest as a system of ideas embedded in the structure in the form of best practices for controlling tobacco for adoption into domestic tobacco 
control laws. Consequently, some policy makers and societies are willingly adopting the best practices as domestic policy while others are indirectly being coerced by the tobacco control network actors to adopt the policies. The actors offer different kinds of technical and financial incentives to motivate the adoption of the laws. Moreover, the knowledge transfer actors governmental and nongovernmental actors like the WHO, ACS, the Commonwealth, the Bloomberg Foundation and Initiative, the Union, IDRC, RITC, media practitioners, and individual activists, among others- are actively spreading the message on the health hazards of tobacco and the adoption of their ideas as the best practices to halt the hazards.

The collaboration between these actors and their daily interactions with countries that have not yet adopted comprehensive tobacco control laws results in a change in the way that the tobacco issue was originally understood or perceived. This culminates in the adoption of domestic tobacco control laws or internal pressure on the policymakers to adopt such laws. Therefore, the trans-border advocacy network, which combines different approaches and perspectives, helps to undertake a comprehensive investigation of the determinants of policy change in the countries. The approach helps to understand why a country that considered tobacco production as essential bedrock of its economy may decide to diversify the economy to make it less dependent on the tobacco product. It also helps to identify the nature and sources of information influencing the policy adoption as well as the possible incentives directly or indirectly influencing the adoption of the FCTC instruments as domestic tobacco control laws. 


\section{Figure 3.1: Trans-border Advocacy Network and Tobacco Control Policy Change}

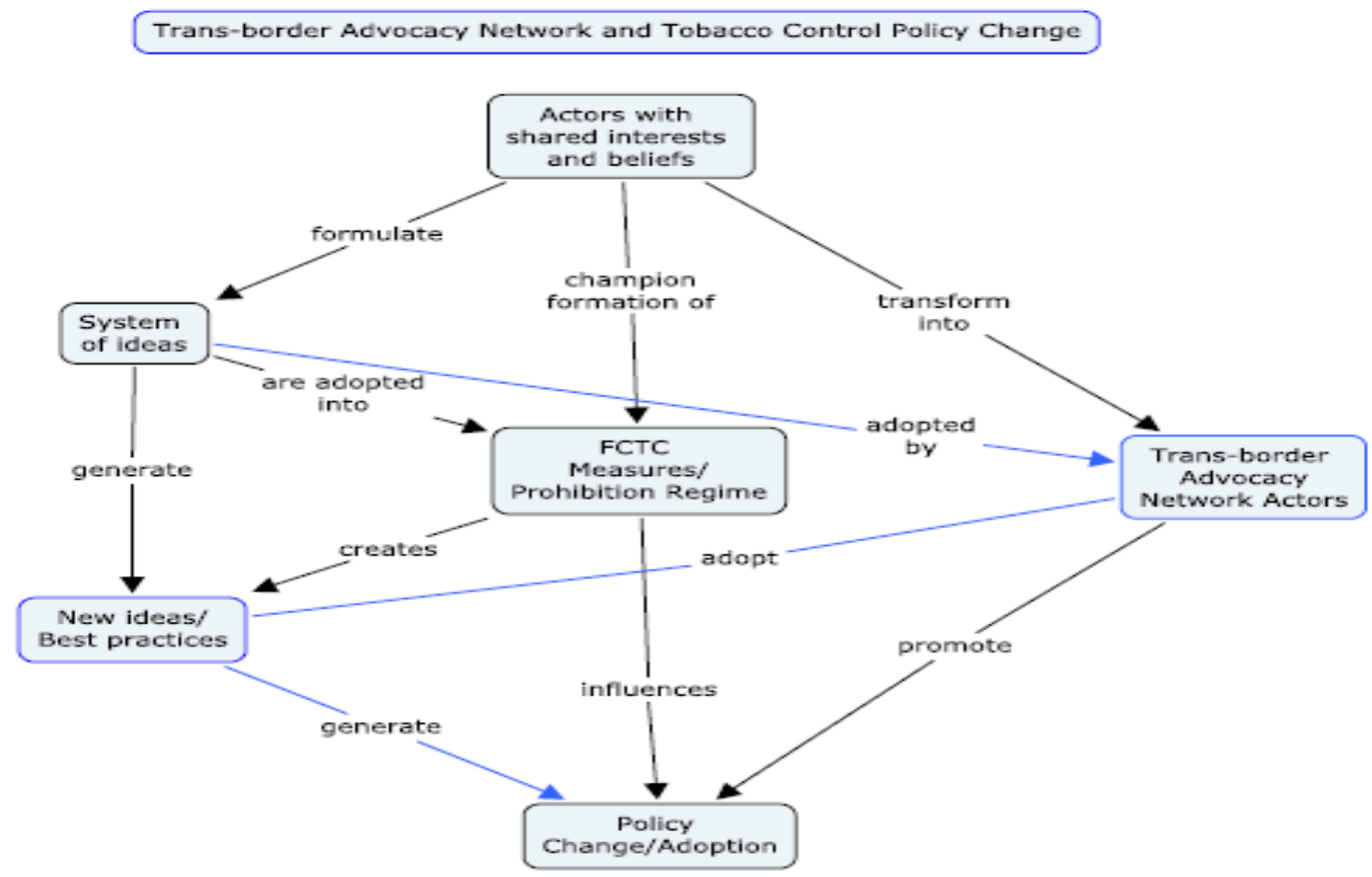

Source: (Self developed model based on the studies of Mamudu 2005; Ladi 2005; Keck and Sikkink 1998, Finnemore 1996, McNamara 1998)

\section{FCTC Instruments and Tobacco Control Policy Change}

The central issue in the tobacco control policy subsystem is the adoption of the FCTC instruments and best practices as domestic tobacco laws. Whiles the tobacco industry network has been actively obstructing the adoption of these instruments, the international tobacco control network (ITCN) continuously promotes the adoption of the instruments as domestic tobacco laws in the developing countries to protect public health and halt the activities of the transnational tobacco companies. In spite of the variations in the categorization of the policy instruments by scholars, the tobacco control instruments are generally summarized under the regulatory (advertising, sales, environmental tobacco smoke, regulations of ingredients), capacity building (funding for community development programs to combat tobacco use), Finance or economic 
incentive (taxation and other levies, agriculture incentive, litigation against tobacco companies for financial recovery of healthcare cost and lost taxation through smuggling), education (health warning label, general anti-smoking and anti-tobacco use campaigns), and learning tools (legislative and executive reports) (Studlar 2002: 221-222, 2003, 2004; Schneider and Ingram 1997; Licari 2002; SEATCA 2011). Similarly, the FCTC groups the instruments into demand and supply reduction measures (WHO-FCTC 2009).

\section{a) Price and tax instruments}

An increase in taxes, which generally leads to a rise in the price of tobacco products, is considered one of the effective means of controlling the consumption of tobacco (Studlar 2004). For smokers in many developing countries who have meager income, a high price of tobacco could serve as the red light to end the smoking of cigarettes in spite of the addictive nature of tobacco (WHO 2008; Studlar 2004). A decrease in the income of such smokers will compel them to examine their choices and focus more on basic necessities. A reduction of about $3 \%$ in consumption could result from an increase in the price of tobacco products by $10 \%$ (Jha and Chaloupka 2000; SEATCA 2011). In addition, the increase in the price of tobacco products through taxation could generate more revenue for the governments in the developing countries (World Bank 1999, Jha and Chaloupka 2000, Studlar 2004, WHO 2008). However, the negative side to price increase is the possibility for increased smuggling and illegal trading of tobacco from neighboring countries. But the countries could offset these illegal trades by instituting some form of litigation against perpetrators of such activities as was done by the US and Canada (Studlar 2004). 


\section{b) Protection against secondhand smoke or "passive smoking"}

Secondhand smoke or passive smoking is the process whereby nonsmokers are exposed to tobacco smoke against their will. Inhaling such smoke creates a lot of health hazards to the nonsmokers even though they chose not to smoke. Therefore, the adoption of laws that can prevent nonsmokers from being exposed to cigarette smoke is considered essential, not only to protect their health, but to ensure some sort of justice for the nonsmokers who are forced to unintentionally inhale what they have not consented (SEATCA 2011). Pioneered by Anglo-American countries, these laws have become one of the core measures for tobacco control globally as many countries have adopted different aspects of such laws (Studlar 2004). Generally, the law places restrictions on smoking in public places, such as restaurants, public transport, government buildings, airlines, and gaming facilities. Countries like Australia have use litigation against the tobacco companies as means of enforcing their second hand smoking instruments (Studlar 2004).

\section{c) Product regulation and ingredient disclosure}

The regulation of cigarette products and disclosure of ingredients used in making the cigarette will help the smokers to have an idea of the health risk associated with their choice to smoke and possibly serve as a deterrent to smoking. The regulation requires the producer to indicate as clear as possible the levels of tar and nicotine used in the tobacco products, and the possible diseases that the consumption of the product can cause, as well as information on cessation (Studlar 2004; MOH, GHS \& FCTC 2010). Studlar (2004) maintains that the warning labels on cigarettes packs is visible sign of tobacco control. 
The ingredients disclosure and placement of warning label instruments started in the United States in the 1965 but many countries have since adopted different and stricter form of the instruments, which stresses the color, size, position, nature and content of language used in the warning (Studlar 2004, WHO 2008).

\section{d) Restrictions on advertising, promotion, and sponsorship}

Advertising, promotion and sponsorship is considered as one of the effective means of luring people to engage in certain behavior. In the case of smoking, the TTCs have been known to use attractive and deceptive advertisements to attract new smokers (WHO 2008, Mamudu et al 2008). For many countries, especially the developing ones, ban on advertising has only focus on direct television and radio advertising, leaving the tobacco companies to adopt other forms of advertising such as billboards, print and point of sale advertising (Studlar 2004, Munthali 2009). Point of sale advertising is when sellers of tobacco products place their products in advantageous places to induce or to attract consumers. Studlar (2004) argues that in some instances, advertising that comes with international sponsorships are ignored as the emphasis of control has been on domestic advertising. Recently, emphasis has been placed on banning all forms of tobacco advertising and promotions to halt the increasing trend in the rising number of new smokers as a comprehensive means of controlling the deceptive activities of the TTCs. Already certain jurisdictions and localities in the United States have started to control the point of sale advertising of tobacco products and certain countries in the EU are controlling all the other forms of advertising (Studlar 2004, Asare 2007). Some countries 
also regulate promotional activities like placing of tobacco names on other products, giving of free samples, and smaller packets to children.

e) Education of the public on the consequences of smoking

Education helps people to make informed decisions, and in many cases the lack of knowledge on the consequences of certain actions may cause people to take decision that may be detrimental to their wellbeing. It is generally believed that most smokers are unaware of the health consequences of smoking, and therefore an education program to that effect will help to reduce the number of smokers. The education is to highlight the harm associated with smoking for nonsmokers and cessation programs for smokers. Sometimes the images of harm caused by tobacco to people in other areas are used to deter prospective smokers. Information on the harm caused by the growing of the tobacco leaves and alternative economic activity for tobacco growers are offered to the farmers (WHO 2008; FCTC 2010).

\section{f) Inclusion of tobacco cessation services in national health programs}

Cessation services are considered essential to assist smokers to quit smoking. Evidence suggests that many times smokers try to quit but go back to smoking. Therefore, the availability of cessation services will prevent relapsing on the attempt to stop smoking and help smokers to quit. A national cessation program can boost the morale of smokers and encourage them to fight the grip of nicotine during the attempt to quit smoking (FCTC 2010). 
g) The establishment of a national coordinating mechanism for tobacco control

A national mechanism for coordinating tobacco control will help to adopt policies that are effective and relevant to the tobacco control situation of individual countries. Even though countries are implored to adopt the FCTC measures, the countries are required to adopt measures that are relevant to their need, and the presence of a national coordinating mechanism will help to facilitate that. The countries are supposed to spearhead the capacity building activities through which programs are established to develop the skills of the locals in addressing the problem of concern. The government can also champion activities to mobilize support for tobacco control among the people by setting a positive tone to support the tobacco control efforts (FCTC 2010).

h) Prohibiting smuggling or unlawful trade in tobacco product and liability Smuggling, or unlawful trade in tobacco, has been one of the means through which TTCs make tobacco products available for domestic consumption in the developing countries. Therefore, in addition to the provision of more resources and personnel for enforcing tobacco control measures, an effective mechanism to prevent smuggling and unlawful trade is necessary for any effective tobacco control measures to succeed. Many of the developed countries like the US and Canada have used litigation as means of preventing illegal trade and smuggling (Studlar 2004). Therefore, such a strategy could aid the developing countries to curtail illegal trade and smuggling of tobacco into their countries. 


\section{i) Prohibition of the distribution or sales of tobacco to under age people}

The recent strategy adopted by the TTCs is to induce minors or under age children to smoke, with the belief that once they get hooked to the product, they will continue to smoke all of their life (WHO 2008). In addition, the companies realize that under age people are easy to lure into smoking. The adoption of the minimum age requirement for smoking has been in place in certain countries since the early part of the $20^{\text {th }}$ century but has not been effectively implemented. Studlar (2004) maintains that some countries started to change the age limit to 18 and 19 years in the 1980s and used stringent measures like inspection and heavy fines to enforce it. Therefore, the adoptions of policies that will prevent the sales of tobacco products to minors can go a long way in controlling the level of tobacco consumption in the developing countries.

\section{j) The provision of support for economically viable alternatives}

Many developing countries grow tobacco leaf because of the contribution of this product to their GDP. Tobacco production serves as the main source of employment for over $50 \%$ of their population. The provision of a viable alternative will help galvanize support of tobacco control policies in those countries as it will provide employment for the people and income for the country (WHO 2008).

The international tobacco control network actors believe that the adoption of the FCTC instruments and best practices as domestic policies could help to curtail the anticipated health hazards associated with tobacco consumption in the developing countries. Therefore, they are enforcing the norms of the global tobacco control prohibition regime by ensuring that the 
developing countries who have not adopted effective tobacco control measures will comply with the requirement of the FCTC.

\section{Developed Countries, Global Tobacco Prohibition Regime, and Developing Countries}

Nadelmann (1990:485) maintains that the emergence of a prohibition regime is characterized by the establishment of international institutions and conventions that serve as a coordinating body for the enforcement of the norms of the regime. As indicated earlier, the birth of the WHO-FCTC convention finalizes the establishment of the global tobacco prohibition regime. The FCTC requires all member countries to translate it provisions into national laws that are effectively implemented (Cairney et al 2012). Moreover, the FCTC influences the behavior of developing countries and places specific regulations on them in way that is similar to "hard law". Additionally, the developing countries are obligated by the guidelines set by the Conference of Parties and the FCTC ratification and implementation processes (Cairney et al 2012). The transnational tobacco control actors are actively promoting compliance with the tobacco prohibition regime in the developing countries to proscribe the activities of the tobacco industry network and also to halt the tobacco health hazards. In sub- Saharan Africa, the actors use knowledge transfer and learning processes, bilateral and multilateral relations, ideas, and network to shape the behavior of the countries and also to ensure that the countries implement effective tobacco control measures (Cairney et al 2012).

The tobacco control unit of WHO, Tobacco Free Initiative, promotes the development of national policies and programs, advocate for tobacco-free societies and non-use of tobacco, and serves as a clearinghouse for scientific information on tobacco use and control (Cairney et al 2012; WHO 1988e; WHO 2005). The TFI through the six regional offices of the WHO and 
country representatives, offer advice and technical knowledge to the developing countries to enable them adopt effective domestic tobacco control laws (WHO 2009). Another strategy of the WHO in promoting the adoption of the FCTC protocol is the use of education and campaigns to sensitize the countries and the public of the dangers of tobacco consumption. As indicated earlier, the TFI uses the Annual No Tobacco Day to highlight the harm associated with tobacco consumption as means of preventing the consumption of the products. Moreover, the provision of scientific evidence linking tobacco to certain health hazards helps to convince the countries and individuals about the harm of tobacco that helps to prohibit the use of the products.

Other international governmental organizations such as the World Bank, United Nations, and the Commonwealth also play key role in enforcing the tobacco control prohibition regime. The World Bank currently prohibits the use of its agricultural loan for growing tobacco (Asare 2007) and also promotes the economics of tobacco use and control (WB 1999; Jha \& Chaloupka 1999; Mamudu \& Glantz, 2009; Cairney et al 2012). Also, the Ad Hoc Interagency Task Force on Tobacco created by the UN in 1999 as a focal point on Tobacco Control helped to familiarized the developing countries with the fundamentals of the scientific evidence and arguments associated with tobacco use and control (Shafey, et al 2009; WHO 2008; Cairney, et al forthcoming). With time, the developing countries have increased their interest in tobacco control, especially in the areas of health education and using warning labels to create awareness of the health effects of tobacco (Cairney et al 2012; Interview 2011). The Health Ministers Conference of the Commonwealth also recommends resolutions for controlling tobacco, which are often adopted by the Meetings of the Heads of Governments of the member countries (Krishnan et al 2003; Latif 2004; Commonwealth Secretariat 2007). 
Other means through which the developing countries experience the enforcement of the tobacco control prohibition regime are bilateral and multilateral relations, which serve as sources of learning and channel of diffusion of tobacco control knowledge (Cairney et al 2012). Cairney et al (2012: 13) maintain that the US Centers for Disease Control and Prevention has since the 1999 collaborated with the WHO and other governmental and nongovernmental organizations in Canada, UK, and U.S. to launch the Global Tobacco Surveillance System (GTSS) to systematize tobacco use and control data ( also see WHO 2005; CDC 1999). They argue that the Global Youth Tobacco Survey (GYTS) was conducted in 162 countries between 1999 and 2008 for all the six regions of the WHO, and a total of 165 tobacco control research projects funded by organizations and institutions based in the developed countries were undertaken in several countries of the six regions (also see Warren et al 2009; Mackay et al 2006). Cairney et al (2012) argue that the engagements have generated a lot of tobacco control research in the developing countries in recent decade, which has given policy makers easy access to local evidence on tobacco use and control, and also made public health as the focus of policy choice.

In addition, philanthropic organizations and other nongovernmental organizations from the developed countries have been collaborating with the WHO and governmental organizations to promote tobacco control ideas to the developing countries (Cairney et al 2012). Cairney et al (2012: 14) argue that these organizations propagate three main ideas: (1) the shift of tobaccoinduced morbidity and mortality to the developing countries; (2) socio-economic costs of tobacco use; and (3) tobacco industry target of developing countries as means of influencing the behavior of the developing countries. They further argue that the notion that tobacco companies have shifted their activities to the developing countries, and that $70 \%$ of the tobacco epidemic will be from the developing countries by 2030 if effective tobacco control policies are not 
adopted has compelled some policymakers of the developing countries such as South Africa to adopt effective tobacco control law (also see Jha \& Chaloupka 1999, 2000; Mamudu \& Glantz 2009; Asare 2009).

Mamudu and Glantz (2009) note that the African countries indicated the tobacco-induced morbidity and mortality as the core issue for their support of the FCTC during the FCTC deliberations. In addition, the availability of increasing evidence that the healthcare cost of tobacco use and the indirect cost resulting from lost productivity and other social dislocations negatively affect the developing countries have shifted the political-economic to public health focus of policy making in the developing countries (Barnum 1994; Panos Institute 1994; Cairney et al 2012). The combination of these activities and strategies has been the mode used by the transnational tobacco control network, which is serving as the core agents, to ensure compliance of the developing countries with the tobacco control prohibition regime.

\section{Transnational Actors and Tobacco Control Knowledge Transfer in the African Region}

The belief among knowledge transfer agents is that it is easier to collectively engage the countries in the African region to assist them with the adoption of best practices as domestic tobacco laws because of the similarities of the tobacco control in the region. This has resulted in a strategy whereby the agents engage the countries collectively to transfer tobacco control knowledge to them (Interview 2010). An interviewee from the American Cancer Society indicated that collectively engaging the countries helps to ensure that all the countries can benefit from the meager resources and also be united in halting the activities of the tobacco industry on the continent. He indicated that when it comes to areas where the 
tobacco control situation of individual countries are unique, funding have been provided directly to domestic NGOs to campaign for that purpose.

In general, many of international NGOs and IGOs have provided technical, financial, informational, and capacity building support to governmental institutions, activists and NGOs both at the national and regional levels. For instance, the WHO, which has been at the forefront of promoting tobacco control globally, uses educational symbolic politics like the annual World No Tobacco Day celebration to highlight the health hazards of tobacco and promote the adoption of tobacco control laws. The event is very popular in the developing countries and it is celebrated in places where there is no tobacco control program or activity (Cairney et al 2012). The World Health Organization also organizes periodic training and conferences for tobacco control actors (mostly in the health sector), to offer them the technical expertise and advice for tobacco control.

The Commonwealth of former British colonies uses the Health Ministers' meetings to champion the adoption of tobacco control laws by member countries. It sponsors studies to examine the impact of tobacco control on the member countries, especially the low and middle income countries (Latif 2004, Krishnan 2003). Latif (2004) argues that the objectives of the studies were to provide recommendations for assisting members that have not adopted comprehensive tobacco laws. Realizing a direct link between tobacco consumption, poor health, and poverty, member countries see tobacco control as a necessary measure to achieving the Millennium Development Goals (MDGs), which were adopted by the Commonwealth Head of Governments Meeting (CHOGM) held in Coolum, Australia in March 2002 (Latif 2004; Krishnan 2003, Commonwealth Secretariat 2007). 
Some of the recommendations presented in the reports included the idea that the Commonwealth should share expertise on tobacco research, legal skills, and also ensure that BAT, which operates in almost all the member countries, maintain the same operational ethical standards internationally as it does in the UK (Latif 2004, Commonwealth Secretariat 2007). According to Latif (2004), some of the recommendations advised the Commonwealth members to task its legal division to come up with a model tobacco bill based on the best practices in the developed member countries for adoption by the developing members to save them resources and also protect public health. So far, the Commonwealth play advisory role for member countries by offering important information on who to contact for tobacco control knowledge (Commonwealth Secretariat 2007). Mostly, the Health Ministers decide on resolutions which are adopted by the meeting of the Heads of Government. The conferences of the Commonwealth Health Ministers have become one of the venues where member countries share knowledge about the best practices of tobacco control. For instance, organizations such as the International Development and Research Center of Canada and Canadian Public Health Association (CPHA) have use the conferences of the Commonwealth Health Ministers to champion the signing of the FCTC protocol and adoption of domestic tobacco control laws by the member countries (IDRC, 2003; CGTCF 2006).

Also, the Commonwealth Health Ministers Conference played a crucial role in the activities of the Canadian Global Tobacco Control Forum (CGTCF), a multi-agency consortium for which the Canadian Public Health Association (CPHA) is the coordinating and administrative agency (CGTCF 2006). The CGTCF, with the support of the Commonwealth Health Ministers Meeting and the FCTC Conference of the Parties (COP), undertook regional action towards the 
implementation of FCTC and organized activities that focused on tobacco control alliancebuilding in Burkina Faso, Congo-Brazzaville, Mozambique, and Niger as well as a capacity building training in Cuba, Brazil, Peru, Mexico, and Colombia. The Forum provided financial and technical support to non-governmental organizations (NGOs) and government partners in various key countries and regions to enable them benefit from Canadian expertise to achieve ratification of the FCTC and/or implementation of FCTC measures (CGTCF 2006).

The Meeting of the Commonwealth Heads of Government held at Trinidad and Tobago in 2009 identified tobacco consumption as one of the major factors of non-communicable diseases (NCDs) that is a huge public health problem for all the member countries (Commonwealth Secretariat 2011). Therefore, they agreed to reduce the incidence of NCDs by adopting different policies and community-based initiatives to discourage tobacco use, unhealthy diets, and to promote physical activity (Commonwealth Secretariat 2011). So far the activities of the Commonwealth have been limited to deliberation and advocating for the adoption tobacco control laws and there is no clear evidence that that the decision has actually led to the adoption of legislation that are well implemented but that cannot be ruled that. However, tobacco control activists maintain that the recognition by the Heads of Government that tobacco cause health hazards, which could be avoided with the adoption of relevant tobacco control laws is a huge step, especially for the developing countries in Africa. This is because such a realization could motivate the political will to promote the adoption of relevant tobacco control laws to protect public health. In addition to the conferences of the Commonwealth, another venue that has been used by actors to promote tobacco control policy adoption with policymakers is the African Union (AU). Organizations such as the Framework Convention Alliance, an association of tobacco control governmental and nongovernmental organizations, have used 
the conferences of the AU to champion the adoption of effective regulatory tobacco control policies. The Framework Convention Alliance (2007) reports that the WHO African Regional Office Coordinator, Dr. Ahmed Ogwell, has attended several conferences of the officials of AU, one of which was the third ordinary session of African Union Ministers of Health Conference held at Johannesburg, South Africa, from April 9-13, 2007.

The Report indicates that the meetings discussed measures to improve the health situation within the continent and recognize the adoption of effective tobacco control legislation as a major component to achieving that objective. It further argues that the ministers adopted a health strategy and a declaration to strengthen the health system for equity and development of the continent at the end of the conference. The meetings and declarations are important because domestic tobacco control activists can use that as accountability politics to put pressure on their respective governments to abide by their commitments by formulating and adopting tobacco control legislations. The Framework Convention Alliance has championed and facilitated the establishment of the Africa Tobacco Control Alliance (ATCA), which is serving as a Pan-African network for tobacco control for the promotion of health and a tobacco free Africa (ATCA 2009). The Africa Tobacco Control Alliance received a grant of US \$10,000 from the FCA for its first board meeting in Nairobi, Kenya in April 2009 that enabled the organization to identify five priority areas of activities to focus on in the next five years (ATCA 2009). The Africa Tobacco Control Alliance (2009) indicate a receipt of a grant from IDRC in the sum of CAN \$ 122,000 to establish a secretariat and expand its activities to attract more audience and support. In addition, the Norwegian Cancer Society (NCS) granted US $\$ 50,000$ to the ATCA to develop and distribute materials that indicates the link between tobacco control, 
poverty alleviation and development (ATCA 2009). The ATCA used the material as the basis for lobbying government officials for the inclusion of tobacco control in the poverty alleviation strategies and developmental plans of the governments. The Alliance organizes exhibitions and side events during meetings and conferences of regional and sub-regional bodies of the officials like the African Union, ECOWAS, SADC, EAC, and ECCAS (ATCA 2009).

The Bill and Melinda Gates Foundation also offer grants to promote tobacco control in the African region. The Foundation has given the American Cancer Society a grant of $\$ 7$ million over five years to coordinate and lead evidence-based tobacco control approaches in controlling the consumption of tobacco and its related health problems in the African region (Becker, 2010). Becker (2010) maintains that the intention of the grant is to enable the Society to organize and assert leadership of the Africa Tobacco Control Consortium to curtail the tobacco epidemic. The Consortium is made up of the Africa Tobacco Control Regional Initiative (ATCRI), Africa Tobacco Control Alliance (ATCA), Framework Convention Alliance (FCA), and Campaign for Tobacco-Free Kids (CTFK), and the International Union against Tuberculosis and Lung Disease (The Union). Many tobacco control organizations and activists from the region have also benefitted from a joint grant of $\$ 500$ million provided by the Bill and Melinda Gates Foundation and the Bloomberg Foundation for global tobacco control initiatives ${ }^{16}$ (Interview 2010/2011). The grant from the Bloomberg Foundation and Initiative is jointly managed by the International Union against Tuberculosis and Lung Disease (The Union), and the Campaign for Tobacco Free Kids (Interview 2010/2011). An interviewee from the Bloomberg Foundation maintains that the disbursement of funds for tobacco control activities are normally channeled through five major organizations - the

\footnotetext{
${ }^{16}$ For details about the grant, visit http://www.gatesfoundation.org/press-releases/Pages/bloomberg-gates-tobaccoinitiative-080723.aspx retrieved on 5/4/2010.
} 
World Health Organization, Campaign for Tobacco Free Kids, Centers for Disease Control and Prevention Foundation, the World Lung Foundation, and John Hopkins University Bloomberg School of Public Health - to countries and tobacco control agencies.

However, funding has been directly offered to certain organizations to undertake tobacco control activities in countries considered priority areas such as Indonesia (Interview 2010/2011). The interviewee indicated that the total commitment of the Foundation for tobacco control is US $\$ 375 \mathrm{~m}$, to be disbursed over 6 years starting from 2007. She further said the Foundation has so far disbursed about US \$ 250 million, averaging about US \$ 58.5 million a year. She indicated that the Gates Foundation joined the Bloomberg Foundation in 2008, and the Gates Foundation has so far offered US \$15 million in support of the tobacco control activities. The participants said that Michael Bloomberg (business entrepreneur and mayor of New York City), believes that helping other countries control their tobacco situations indirectly help New York City because the city attracts people from all over the world. In that sense, if other countries succeed in halting tobacco and cigarette smoking, the city might likely have fewer people from other countries that smoke and be in a position to focus on the smokers already in the city. Moreover, tobacco control activists from Ghana and Malawi are among the individuals that have benefitted from a grant of $\$ 10$ million provided to vulnerable countries by the American Cancer Society (ACS), the International Union against Cancer (UICC), and the Cancer Research UK to create awareness about the dangers of tobacco, and to put pressure on their governments to sign and ratify the FCTC (ACS, UICC and Cancer Research UK, 2004). The American Cancer Society and Cancer Research UK also offered funding to the Africa Tobacco Control Regional Initiative to provide the stage for sharing relevant information and provide institutional support and 
capacities building to adopt, implement, and enforce efficient tobacco control laws and programs in Africa ${ }^{17}$.

The Research for International Tobacco Control and the International Development Research Center of Canada also established the Africa Tobacco Situational Analysis (ATSA), with the support of the Bill and Melinda Gates Foundation in 2007 to analyze the tobacco control situation of certain African countries, ${ }^{18}$ including both Ghana and Malawi. The ATSA helps to build the capacity of researchers to gather, synthesize, and analyze relevant tobacco control information as well as to use the information generated in supporting broader strategies for tobacco control in Africa. The Canadian International Development Research Center (2009) maintains that the objective of the ATSA has expanded to include the use of country specific case studies that analyze and document the broader social, economic and political tobacco control activities as way of identifying policy priorities and interventions. The twelve countries adopted for analysis include Malawi, Ghana, South Africa, Senegal, Cameroun, Eritrea, Tanzania, Zambia, Kenya, Mauritius, Burkina Faso, and Nigeria. Generally, anti-tobacco network campaigns highlight the negative effects of smoking on public health, lobby for the ratification and signing of the WHO-Framework Convention on Tobacco Control, and draw attention to the health hazards of tobacco products and negative activities of tobacco companies. The network also build coalitions to pressure the government to adopt effective tobacco control laws, mobilize periodic demonstrations against the tobacco companies and the government, liaise with other transnational actors to provide updated information on the health hazards of tobacco, and encourage government (especially those relying heavily on tobacco) to diversify their

\footnotetext{
${ }^{17}$ See http://www.atcri.org/eng/index.php for details.

${ }^{18}$ See the IDRC website for more information http://www.idrc.ca/en/ev-119375-201-1-DO_TOPIC.html
} 
economy. The actors promote different types of tobacco control instruments for adoption in the developing countries and some of the details will considered in the next chapter.

\section{Anticipated Tobacco Control Policy Change in the Developing Countries}

The study adopts the broader view of policy to track the changes in the policy behavior of the countries. The broader sense of policy change could involve the change of behavior or action on a certain issue as well as adopting a policy that indirectly addresses a problem within the domestic environment. Sometimes policymakers and politicians may feel reluctant to adopt a direct policy for addressing a particular problem because of the perceived impact on their political capital or the economy of the country. Therefore, they may adopt another policy that indirectly addresses the problem but is seen to be a safer policy to adopt for the policy makers. A scenario like this in tobacco control could involve the refusal to adopt a direct tobacco control law because of the anticipated impact of such laws on the gross domestic product of the country but adopting other policies such as labor, environment, or economic diversification policies to make the country less reliant on tobacco products. The adoption of such laws may indirectly help to protect public health by protecting nonsmokers, especially children from exposure to nicotine. In another scenario, a country may change its behavior from initially refusing to sign and ratify the FCTC protocol to signing the protocol but not ratifying it. Moreover, the rhetoric of the politicians could move from speaking negatively about the tobacco issue to being neutral or speaking a little positive about the issue in question.

Many developing countries have agrarian economies and depend on tobacco as the single major cash crop, which contributes substantially to their gross domestic product and also serve as a major source of employment for the majority of the citizens. In that sense, politicians may be 
afraid of adopting policies that could create unemployment for the people and also decrease the contribution to the gross domestic product. For instance, tobacco contributes a major percentage of the gross domestic products of countries like Malawi (Otanez et al 2009, Cairney 2007). The sector also accounts over $70 \%$ of the foreign earnings of the country. Moreover, policymakers may often resist the idea of adopting a stricter tobacco control law that could create unemployment and incur the displeasure of the public. In addition, the decision not to adopt tobacco control laws may be to avoid losing the sponsorships and contributions that the tobacco companies offer to the activities and campaigns of influential politicians (Interview 2010). Nonetheless, the unwillingness to adopt such laws may be because of the fear of losing their support or attracting resistance from the tobacco interest groups like the local tobacco farmers who are used by the tobacco companies to lobby the government against adopting stringent tobacco laws.

Many times the public resistance to the adoption of tobacco control laws may be due to the picture painted about the importance of the product to their economic wellbeing. In such situations, people may change their minds to support the adoption of relevant tobacco laws when they get other information on the health hazards of the product through the activities of NGOs, IGOs and tobacco control activists. Moreover, the availability of relevant information on the health implications of tobacco could influence the behavior of the politicians and policymakers, who may soften their stand towards the adoption of laws on tobacco due to pressure from international and domestic activists as is evidenced in Malawi ${ }^{19}$. Sometimes, international and regional pressure may cause a government to adopt certain policies that will improve their image in response to the way that the tobacco issue is framed by the tobacco control activists and

\footnotetext{
${ }^{19}$ A detail discussion on how the political authorities of Malawi has soften their stand on tobacco control is done in chapter five
} 
interests. Moreover, donor countries and organizations may use grants as incentive to influence the adoption of certain laws (Evans 2004). It must be noted that a key strategy in the struggle to control tobacco products has been the way the issue is framed (Studlar 2002, Cairney 2007, Cairney et al 2012). The tobacco industry network always tries to project tobacco as positive for the economies of the developing countries while the anti-tobacco network associates the product with its health hazards. The success of the activities of any of these networks and interests has great impact on the actions of the policymakers and the public.

\section{Conclusion}

The chapter has broadly examined the factors that influence policy change such as the actions and motivations of transnational knowledge transfer actors, their strategies of operation, and power structure. In addition, the notion of policy change in the tobacco control policy subsystem as perceived by this study has been generally examined. Moreover, the activities of the actors in the tobacco control networks to prevent or promote the adoption of stricter tobacco control laws have been reviewed. The chapter also examined the activities undertaken by transnational tobacco control actors to control tobacco in the African Region. The finding is that many of the transnational actors and organizations engage the actors from the developing countries at the regional level to spread the meager resources and also adopt collective action for addressing the tobacco problem. The study concludes that paying attention to a broader view of policy change and using it as the basis of analyzing the politics and process of tobacco control in Ghana and Malawi will help to understand the political struggles that occur during the process of adopting tobacco control law. 


\section{Chapter Four}

\section{Efforts to Control Tobacco in Ghana}

\section{Introduction}

This chapter examines the efforts to control the consumption of tobacco products and its health hazards in Ghana. Tobacco came to Ghana after World War II when the transnational tobacco companies moved to this part of the world (Cairney et al 2012). Since then Ghana witnessed the activities of several tobacco manufacturing companies for about two decades. Nevertheless, the country has some tobacco control activities going on in effort to curtail the consumption and the harm associated with tobacco.

This chapter examines the tobacco control activities of Ghana focusing on the political struggles and resistance within the process. It looks at the composition of the tobacco control interest groups and actors who are directly or indirectly involve in the process. In addition, it examines the manner through which tobacco control knowledge is transferred and how relevant information is used to change the understanding of the tobacco issues in the country. In other word, it examines how the trans-border advocacy network approach helps to explain the process of tobacco control in Ghana focusing on the activities of anti-tobacco advocacy network to hasten the adoption of appropriate tobacco laws and strategies adopted by the tobacco industry and its affiliates to prevent the adoption of such laws in Ghana.

\section{Tobacco and the Ghanaian Economy}

Ghana has agrarian economy and has depended on major cash crops as the key contributors to her GDP. Tobacco at certain periods was one of the major cash crops and tobacco leaf farming provided raw materials for the domestic cigarette manufacturing plants in the 
country. This facilitated the export of tobacco leaves and Ghanaian manufactured cigarettes to the neighboring countries. Additionally, it led to the establishment of a state-owned tobacco leaf buying company and tobacco manufacturing plant after independence in 1957 . However, the last tobacco manufacturing plant in Ghana, operated by the British American Tobacco company, closed its manufacturing plant in 2006 and relocated to Nigeria. Some tobacco control activists believe that the company was forced to relocate because of the unfavorable environment created by tobacco control activities, but the company indicated a strategic business restructuring as the reason for leaving (Interviews 2010). An interviewee indicated:

in Ghana, there are no tobacco advertisements at the moment. Sometimes ago the tobacco industries were supporting beauty pageants etc., those days are over. Public awareness has been made through the GHS, Health Promotion unit and other agencies but they complain of resources for public education. During the World No Tobacco Day celebration you will also find a few media campaigns but beyond that nothing is happening. I met one of the top people in the BAT, who said to me that they folded up in Ghana largely because of the campaign that was going on to get people to stop smoking cigarettes and the use of other products. I was told that it was strategically more economical to send the manufacturing to Nigeria and import into the country ${ }^{20}$

Ghana still produces and exports tobacco leaves and was ranked $74^{\text {th }}$ in tobacco leaf production in the world in 2007 (Wellington et al 2011). In spite of this, the activities of tobacco control advocacy groups have led to the institution of certain voluntary agreements and alternative measures to control tobacco through administrative directives issued by various governmental ministries spearheaded by the Ministry of Health directive to ban smoking in all government facilities and also to ban tobacco advertisement on TV and print media adopted in the 1980s (Owusu-Dabo et al 2010). The administrative directives have achieved minimal gains because of the good will of the public to respect them as means of controlling tobacco in the

\footnotetext{
${ }^{20}$ Adopted from the data of interview conducted by Owusu-Dabo et al 2010
} 
country (Interview 2010). This is in spite of the fact that Ghana at one point had a state-owned tobacco manufacturing plant and tobacco leaf buying company.

Although the National Parliament of Ghana has not passed any tobacco legislation, Ghana has created certain alternatives through executive action that fulfill some obligations of the FCTC measures (MOH, GHS \& FCTC 2010). These include existing laws, regulations and administrative directives issued by different government ministries (MOH, GHS \& FCTC 2010). Ghana was also among the first forty countries that signed and ratified the FCTC in the early period in 2004 and has played an active role in the activities of the Conference of Parties (COP) of the FCTC (MOH, GHS \& FCTC 2010; Wellington et al 2011; Owusu-Dabo et al 2010).

\section{Tobacco Industry Network in Ghana}

Ghana's commercial experience with tobacco dates back to the post WW II when there was increased demand for cigarette by soldiers who had developed the taste while serving at war overseas (Owusu-Dabo et al 2009). The influence of the tobacco industry network in Ghana is linked directly to the history of the companies in the country. The tobacco industry and its affiliates in Ghana are made up of cigarette sellers, tobacco farmers, companies, businesses, manufacturers and individuals linked to the cigarette selling business. Ghana tobacco activities were mostly influenced directly by the both private and public tobacco companies who had manufacturing plants in the country.

Owusu-Dabo et al (2009) indicate that BAT partnered with local business to establish a depot, which served as a warehouse and a distribution center for the country in 1948, but in 1951, created the Gold Coast Tobacco Company (GCTC) to take over the business. In 1952, BAT established the Pioneer Tobacco Company (PTC) and tasked it to spearhead domestic 
tobacco cultivation and manufacturing, which resulted in the official production of cigarettes in Ghana in 1954 and by 1959; the PTC took over GCTC (Owusu-Dabo et al 2009). Owusu-Dabo et al (2009) also note that the tobacco industry enjoyed significant success from that period, but in 1962 the post- independence government passed a law to take over tobacco marketing in the country as part of Nkrumah's socialist program for economic development.

Owusu-Dabo et al (2009) further indicate that the government established the Ghana Tobacco Leaf Company (GTLC) and purchased the tobacco leaf department of PTC but allowed the PTC to control the management of the new company. However, after the overthrow of the Nkrumah government, the private tobacco companies returned to the business until the Acheampong regime passed another law for the state ownership of foreign companies and organizations in 1976. They maintain that the International Tobacco Ghana (ITG) company was established in 1976 as a partner of Rothmans UK to produce Rothmans King Size cigarette, but the ITG ceased operation in 1989, selling its assets to Meridian Tobacco Companies (MTC), which was jointly owned by state-owned Social Security and National Insurance Trust (SSNIT) and Rothmans UK.

Around the same period, the Leaf Development Company was established in 1988 to produce tobacco leaf for the local market and also for export, which resulted in the first export of 250 tons of tobacco leaf, with export earnings of $\$ 380,000.00$ and also increased the interest in the growing of tobacco in the country. In 1991, Rothmans bought 49.5 percent shares of the company and took over the management of MTC in partnership with SSNIT. In addition, the PTC merged with MTC when BAT bought Rothmans UK, and BAT became a monopoly in the manufacturing of tobacco in Ghana until December 2006 when significant drop in profit and the activities of the tobacco control activists forced the company to cease operation in Ghana and 
relocated its plants to Nigeria (Owusu-Dabo et al 2009, 2010). In spite of the fact that BAT has officially closed their production plant in Ghana, business and agents earlier established to distribute cigarettes in Ghana continue to import cigarettes to the country and also to lobby against the official adoption of domestic tobacco control laws (Interview 2010/2011). The companies that legally import tobacco products into the country at the moment are BAT Ghana, Market Direct, and Target Link (Owusu-Dabo et al 2010). In addition, there are several illegal importers who smuggle the products into and out of the Ghanaian market, and so have a special interest in protecting the status-quo (Interview 2010).

The tobacco industry network also capitalizes on the regional distribution of cash crops to frame the adoption of the comprehensive tobacco legislation as an attempt to kill the economic activities of farmers in the middle belt and the already impoverished northern regions (Interview 2011). This is in spite of the fact that the three regions in the northern part of Ghana -Northern, Upper East and Upper West regions- have the highest prevalence rates of tobacco use 17.7\%, 15.3\%, and 11.4\% respectively (Gyebi, 2007, Wellington et al 2009). The network actors are also alleged to be secretly providing financial incentives to the politicians and policy makers to prevent the adoption of a domestic tobacco control bill (Interview 2010, Owusu-Dabo et al 2010). For instance, there is an allegation that the visit of the President of the British American Tobacco (BAT) to Ghana, after the tobacco control bill was re- drafted to reflect the provisions of the FCTC in 2005, might be the reason why the government dragged its feet to introduce the bill in Parliament for over five years. Since the Ghanaian legislative process requires Cabinet (the executive branch) to introduce all bills to Parliament, tobacco control activists claim that the visit of the BAT President and his private conversation with President Kufour of Ghana was the 
reason why his government failed to introduce the draft tobacco bill to Parliament during his term of office.

In spite of the official ban of the sponsorship of national events by the tobacco companies in Ghana through the directives of the Ministry of Health, the tobacco companies still use other avenues of sponsorship to indirectly influence the politicians. For instance, BAT is one of the sponsors of Chanton House Prize that recognizes the contribution of leaders to economic and social development as well as peace and conflict resolution in sub Saharan Africa. The former

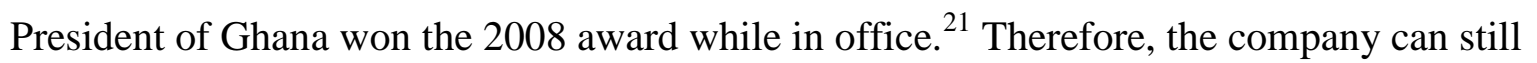
influence the activities of these leaders through their sponsorship of this high profile and prestigious awards. An intriguing twist to the tobacco control story was the announcement by Estelle Appiah, head of the Legislative Drafting Division of the Attorney General Department, that a Public Health Bill incorporating tobacco control provisions had replaced the proposed Tobacco Control Bill drafted by the National Steering Committee for Tobacco Control (Joy News, Monday January 10, 2011).

Mrs. Estelle Appiah maintained that tobacco control is a public health issue and thus incorporating it as part of an overall public health bill will be useful to the public. She also stated that having it as part of the overall public health bill will make it easily accessible to the people. She further argued that the Public Health Bill is divided into eight sections, and the tobacco control provisions are in section six of the bill. Initially, the tobacco control activists were skeptical about the incorporation of the tobacco bill into the Public Health bill. However, the bill has been introduced in the floor of Parliament and it has been referred to the Health Select committee for final amendments (Personal correspondence 2011).

\footnotetext{
${ }^{21}$ For details, see http://www.chathamhouse.org.uk/news/view/-/id/463/
} 


\section{Tobacco Control Network Activities in Ghana}

Ghana has a number of tobacco control voluntary agreements instituted by various directives of the governmental ministries and agencies (Wellington et al 2009; Owusu-Dabo et al 2010; Wellington et al 2010; MOH, GHS \& FCTC 2010). Like many developing countries, the Ministry of Health of Ghana is the main governmental agency, championing tobacco control in collaboration with other NGOs, organizations and tobacco control actors (Wellington et al 2009; Owusu-Dabo et al 2010; Cairney et al 2012). The Research and Development Division of the Ministry of Health currently houses the focal point for tobacco control and it also works closely with the National Tobacco Control Steering Committee (NTCSC) (MOH, GHS \& FCTC 2010). The NTCSC was established in 2002 and tasked with formulating a policy on tobacco control, coordinating all tobacco control activities, and making recommendations for tobacco control under the supervision of the Ghana Health Service (Wellington et al 2009).

The National Tobacco Control Steering Committee is made up of twenty- eight members drawn from several institutions and organizations (See Table 4.1). Owusu-Dabo et al (2009) maintain that the committee replaced the Ghana Committee on Tobacco Control (GCTC), earlier established in 1993 for similar purposes. The Ministry of Health, in conjunction with others government ministries, has issued several administrative directives to prohibit tobacco consumption and its health hazards (Wellington et al 2009; Owusu-Dabo et al 2010; Interview 2010). However, the directives - some of which were adopted before the establishment of the FCTC - are not effectively implemented because enforcement has been through voluntary agreement with stakeholders and the goodwill of the public to respect the directives (Interview 2010, Owusu-Dabo et al 2010, VALD 2010). 
Table 4.1: Composition of the National Tobacco Control Steering Committee of Ghana

\begin{tabular}{|l|l|}
\hline Institution & Number of Representation \\
\hline Ministry of Health/ Ghana Health Service & 4 \\
\hline Developmental Partners & 1 \\
\hline Health Select Committee - Parliament House & 2 \\
\hline Food and Drugs Board & 1 \\
\hline Ghana Standards Board & 1 \\
\hline National Media Commission & 1 \\
\hline Ministry of Trade and Industry & 1 \\
\hline Ghana Tourist Board & 1 \\
\hline Attorney-General's Office & 2 \\
\hline Ministry of Women and Children's Affairs & 1 \\
\hline Ministry of Information & 1 \\
\hline Ministry of Finance & 1 \\
\hline Customs, Excise and Preventive Service & 1 \\
\hline Ministry of Education, Youth and Sports & 1 \\
\hline Ghana Education Service & 1 \\
\hline Ministry of Food and Agriculture & 1 \\
\hline Rep. Coalition of NGOs in Health & 3 \\
\hline Others (researchers, farmers, religious organization, etc.) & 4 \\
\hline Source: Bas
\end{tabular}

Source: Based on Information from Wellington 2007; Owusu-Dabo et al 2010; Interviews 2010

A respondent to an interview indicated that "tobacco advisement on radio and television were banned by the directives of the Ministry of Health long ago. Therefore we seem to be doing very well in the area of controlling tobacco activities. However, there is no official legislation for enforcement" (Interview 2010). The Ministry of Health also banned the advertisement of tobacco in both the print and broadcast media in 1982 (Wellington et al 2009; Owusu-Dabo et al 2010). Also, the government issued an administrative directive to prohibit smoking in government facilities, offices, and public places like cinema centers and restaurants in 1980s (MOH, GHS \& FCTC 2010; Wellington et al 2010). In addition, Ghana also joined the International Quit and Win Program in the same year (Wellington, 2007). The quit and win program uses positive incentives to encourage smokers to quit and has motivated a lot of smokers to quit since it started (Wellington, 2007). Mrs. Edith Wellington - the Principal Health Research Officer of the 
research unit of Ghana Health Service and the focal point person for tobacco control in Ghana indicated at the 2007 plenary session workshop for research for action on the FCTC that the Quit and Win program has seen laudable results since its inception. She maintains that $43.3 \%$ of participants in the program stayed tobacco free by the year 2005.

Ghana also became the $39^{\text {th }}$ country to ratify the FCTC protocol in 2004 when Parliament unanimously voted in support of the signing and ratifying of the international protocol (Kingson 2010; Owusu-Dabo et al 2010; Wellington et al 2009). Additionally, Ghana has played a pivotal role in the activities of the Conference of Parties of the FCTC and has chaired the subcommittee meetings of the conference on two occasions (Interview 2010). The Tobacco Control Steering Committee has been able to come up with a tobacco proposal that is reflective of the FCTC measures and the National Parliament has promised to enact it into law as soon as possible, possibly before the end of their term in 2012 .

Wellington (2007) maintains that the committee members that drafted the bill got briefings on the GYTS statistics on the tobacco situation and the effects of smoking of Ghana, and this knowledge was used in the drafting of the bill. The draft tobacco control bill covers all the FCTC provisions (Interview 2011). The committee used the FCTC provisions and the sample tobacco control policies of countries like Canada, South Africa, Sweden, and Finland, and others, as a guide in producing the draft (Wellington 2007). In addition, tobacco control experts with technical knowledge from domestic and developmental partners such as the WHO, IMF, and World Bank were brought in to make a presentation on the importance of the tobacco control to the country or to assist with drafting the bill (Wellington 2007; Interview 2010).

Moreover, the Ministry of Health has been able to ban smoking in all Ministry of Health facilities since 2006 through other directives (Owusu-Dabo et al 2010; Kingson 2010). Kingson 
(2010) maintains that the Ministry and the Ghana Tourist Board reached an agreement with owners of entertainment and hospitality industry to create a smoke free environment in 2007 . In the same year, other Ministries issued directives to convince people not to smoke in public transport and buildings, ports, stadia, and a designation that some places such as airport, governmental educational institutions, and major hotels were smoke free ${ }^{22}$ (Wellington 2007; Wellington et al 2009; Kingson 2010; Owusu-Dabo et al 2009; 2010). The Tobacco Control Steering Committee also developed a five- year plan of action for controlling tobacco in 2007, which was the first ever plan of action designed specifically for Ghana (Wellington 2007). Wellington (2007) argues that the plan outlines a framework for a comprehensive and a multidimensional public health approach to control tobacco, which primarily focuses on interventionist measures through prevention, cessation and protection.

Owusu-Dabo et al (2010) argue that there is also a minimal achievement in the area of disclosing the content of tobacco and regulation of tobacco products. There is a form that requires the importers of the cigarette to indicate the content of tobacco products they intend to import into the country. The content disclosure and regulations were an outcome of an agreement between the Steering Committee, the Ministry of Health, the importers of tobacco products, and the Ghana Food and Drugs Board, who monitors and enforce the directive. This agreement was to strengthen the regulatory effort of the Food and Drugs Board by ensuring that cigarette imported into the country met the set standard (Owusu-Dabo et al 2010; Interview 2010). However, resources constrain the ability of the Food and Drugs Board to effectively regulate the cigarettes imported into the country, especially those that are smuggled into the country (OwusuDabo et al 2010)

\footnotetext{
${ }^{22}$ Refer to the timeline for tobacco control efforts in Ghana for the details of the administrative directives to control tobacco.
} 
In spite of this, cigarettes imported into the country mostly comply with the FCTC requirements of not falsely promoting tobacco products or deceiving people about the characteristics of the product. The Ministry of Finance has also raised taxes on tobacco products, and an import duty of $140 \%$ of custom insurance freight (CIF) and a value added tax (VAT) of $16 \%$ is now imposed on tobacco imported into the country. The Minister of Health indicated that the flat tax was to be increased to $150 \%$ for health reasons and also to align the Ghana's tobacco tax to international agreement on excise duty (Interview 2011; WHO 2009; Ghana Chronicle, Friday, November 26, 2010). Ghana's total tax on cigarettes is $67.5 \%$ as a proportion of the price of cigarettes, which is fairly high in comparison to countries such as the United States $36.93 \%$, South Africa - 44.28\% -, and Malawi - 53.89\% - but fairly low when compared to countries such as United Kingdom - 76.49\% and Canada - 69.29\% (Tobacco Atlas, 2009). However, the excise tax as percentage of the cigarette price is 14\% (Eriksen, Mackay and Ross 2012). In spite of this, tobacco activists often argue that the taxation is too small to discourage smoking and have called on the government to adopt higher taxes on cigarettes.

An interviewee indicated that the importers comply with the directives of the Ministry to disclose the contents and ingredients of the cigarette even though there is no law to prosecute those that violate the requirement:

The draft bill mandates the FDB to regulate tobacco but since the bill has not been passed into law yet what we do now is just follow the Minster's directive and ensure that all the three importers of tobacco in the country sign a document to ensure that they declare the content of the product they intend to import into the country. At the moment the three companies are BAT, Market Direct and Target link. They are all complying with the demands of the FDB now ${ }^{23}$

The Ministry of Health requires that all cigarettes packs imported into the country should have one of the three rotational health warning of the Food and Drugs Board that reads: (1)

\footnotetext{
${ }^{23}$ Adopted from the interviews of Owusu-Dabo et al 2010 with permission
} 
“smoking causes fatal diseases" (front of pack), "smokers die young” (back of pack), (2) "smoking causes cancer" (front of pack), "smoking damages the health of those around you" (back of pack), or (3) "smoking seriously harms you and others around you" (front of pack), and "stopping smoking reduces the risk of fatal heart and lung diseases" (back of pack). The Food and Drug Board also mandates that the warning must be clear, legible, and written in black and white and also occupy at least $50 \%$ of the most obvious side of the pack (Interview 2011). The officers of Custom, Excise and Preventive Service (CEPS) occasionally seize and destroy cigarette products brought to the country illegally in an effort to enforce the warning requirements and curtail the smuggling of tobacco into the country. For instance, the Northern Sector Command of CEPS destroyed 330,000 cartons of cigarettes in December 2009 because they were either smuggled into the country or had no health warning (GNA, December 13, 2009).

A tobacco control activist indicated that the warning is not enough and that it needs to be in pictures and also bolded so that people can easily see it. He argues that most of the companies do not comply with the $50 \%$ labeling requirement and that the passage of the draft tobacco bill will help to enforce the requirement. Some activists also complained about the inadequate effort to control the smuggling of tobacco products from neighboring countries, the absence of a policy restricting the youth from smoking, and the lack of an effective policy to assist farmers with the provision of viable alternatives ( also see Owusu-Dabo et al 2010, Wellington et al 2009). Although the country is doing very well with creating awareness and educating people about the dangers of tobacco, some activists complained that a lot more could be done as the current effort is limited to the Annual World No Tobacco Day celebrated on the May $31^{\text {st }}$ every year. 
Some activists complain that the adoption of some tobacco control voluntary agreements before the establishment of the FCTC and the early ratification of the FCTC protocol as well as the participation of Ghana in all meetings of the conferences of parties (COP) of the FCTC for deliberating on the implementation of FCTC protocols create the impression that the policymakers have the political will to adopt a comprehensive tobacco control law, but that has not been the case yet (Owusu-Dabo et al 2009). It is for this reason that the Ghana Ministry of Health collaborates with NGOs like the Media Alliance in Tobacco Control (MATCO), Vision for Alternative Development (VALD), Healthy Ghana, Coalition of Nongovernmental Organization in Tobacco Control (CNTC), researchers, media practitioners, and individuals with the passion to end the harmful effect of tobacco on the public health. These actors have collaborated with other regional and international actors to quash the power and activities of tobacco companies and their affiliates in Ghanaians by putting pressure on the government to pass the draft tobacco bill. The groups use accountability politics (Keck and Sikkink 1998) as a means of putting pressure on the government of Ghana to pass the tobacco bill into law. For instance, the director for VALD, Mr. Issah Ali, has constantly reminded the government of its ratification of the FCTC protocol and the obligations that comes with it (Aidoo, 2009) ${ }^{24}$.

The NGOs and the Ghana Health Service use the celebration of the annual World No Tobacco Day celebration to highlight the harm of tobacco to public health and also organize periodic workshops and training to educate the public on the harmful effects of smoking (Wellington 2007; Wellington et al 2009; Kingson 2010). The VALD and CNTC have separately organized tobacco control educational workshop for the public and NGOs, respectively, in Accra (Aidoo 2009). Healthy Ghana also organizes tobacco control workshops for religious groups in

\footnotetext{
${ }^{24}$ Also see http://www.ghanaweb.com/public_agenda/article.php?ID=14155 for more on the related articles.
} 
Accra to solicit their support in the fight against tobacco consumption (Tobacco Control in Africa, 2010). Moreover, VALD recently organized a training workshop for the media to encourage them to be partners in highlighting the public health impact of tobacco through active campaigns and to also improve their reporting on tobacco control and other related issues (Ghana News Agency, December 18, 2009).

To aid the tobacco control effort of domestic actors, certain government agencies and international nongovernmental organizations offer informational, direct training and financial assistance to the local actors and organizations to enable them to put pressure on the policy makers for the passage of the tobacco law. For instance, Ms. Edith Wellington was a beneficiary of the IDRC/RITC Africa Tobacco Situation Analysis Development grant to undertake research aimed at understanding critical determinants of tobacco control in the continent ${ }^{25}$. An interviewee from the Bloomberg Foundation indicated that the Vision for Alternative Development received a grant of US $150,000.00$ in 2009 to undertake a consolidated campaign for the implementation of article II of the FCTC on pictorial warnings on tobacco products and also to promote the passage of the draft tobacco bill into law.

The organization was tasked to advocate for the adoption of a strong national tobacco control bill that stresses pictorial warning. The director of VALD has been very influential in pushing for the passage of the tobacco control bill into law by Parliament (Personal Communication, 2011). The bill has pictorial warning as a major component. The activists believe that even though Ghana has not passed a national tobacco control law, their efforts has chalked up some successes through the voluntary agreement and the directives by the government ministries and agencies. Some believe the current government of the National

\footnotetext{
${ }^{25}$ http://www.idrc.ca/en/ev-119943-201_105232-1-IDRC_ADM_INFO.html for more information
} 
Democratic Party will pass the bill into law before the end of their term of office in 2012, because of the pressure being exerted on the government. The policy makers will pass the bill currently in Parliament, in spite of the replacement of the proposed Tobacco Control bill with the Public Health Bill, which has a provision on the tobacco control instrument in section six of the bill, as previously discussed.

\section{Knowledge Transfer and Tobacco Control in Ghana}

The activities to help Ghana with the adoption of the best practices of tobacco control as domestic law are not very different from what is going on in other developing countries. The international tobacco control actors - nongovernmental and governmental - engage their counterparts in the country in an effort to make Ghana a member of the community of tobacco control countries. The nongovernmental actors, through their interactions with actors from the developed countries, acquire skills and put pressure on the central government to translate the signing and ratifying of the FCTC protocol into the adoption of effective domestic tobacco control laws. The tobacco control agents use incentive to influence the government institutions such as the Ministry of Health and the Ghana Health Service (GHS). Consequently, the Ministry and the Health Service have used the knowledge obtained from interactions with tobacco actors to push for a tobacco-free Ghana through the issuance of directives. In addition, the GHS and tobacco control NGOs and activists have been putting pressure on the Parliament of Ghana to pass a tobacco control bill, backed by law, to curtail the health effects of tobacco consumption.

Normally, the knowledge transfer agents from the developed countries also use other means of sponsorship, direct and indirect engagements, and personal interactions to transfer knowledge. For instance, the Principal Health Research Officer at the Ghana Health Service, 
who doubles as the focal point person for tobacco control in Ghana, was offered an assistantship to go to Canada to train on tobacco control strategies. Basically the training in Canada was meant to expose her to tobacco control policies adopted in Canada and US so as to influence the adoption of similar tobacco control laws in Ghana. In addition, the availability of the literature on smoke free laws, advertising bans, and taxes on tobacco products adopted in countries like Canada, US, and UK influence the Ministry of Health and others in the adoption of tobacco control directives centering on such issues (Interview 2010).

However, the Ghana Health Service officials had to engage the stakeholders to ensure compliance with the directives on the advertising ban, a ban on the sponsorship of sporting events, and voluntarily designating certain areas as smoke free at restaurants and hotels because the voluntary agreement do not have the full force of law. As indicated earlier, the policy culture requires the Parliament to legislate laws for it to have the full force of sanctions. Aside from sponsoring domestic actors for tobacco control programs, the international governmental and nongovernmental organizations also make their expertise available to assist with the formulation of tobacco control policy. The World Health Organization Representative in Ghana was part of the committee that drafted the tobacco control bill (Wellington 2007; Joy News, 2011).

As the umbrella body for the FCTC, the WHO periodically documents the best practices in tobacco control from different countries. Therefore, the advice of a representative of WHO to the committee that drafted the tobacco bill meant easy access to best practices adopted in other countries that could be used in Ghana. In addition, the tobacco control policy ideas borrowed from countries such as Canada, Finland, and South Africa and the advice from tobacco control experts made it easy for the drafting of the tobacco instrument of the country (Interview 2011). Ghana has also attended all the meetings of the World Conference on Tobacco or Health 
(WCTOH) and the Conferences of Parties (COPs) of the FCTC, mostly organized by the FCA. Moreover, Ghana, as a member of the Commonwealth and the WHO, benefits from discussions on effective strategies and measures to control tobacco that takes place at Commonwealth and WHO meetings. For instance, relevant ideas on preventing communicable diseases such as tobacco-related ones are normally discussed in the Commonwealth Heads of Government and Ministers' Meetings and the WHO conferences attended by the President and/or top governmental officials like the Minister of Health and representatives of the Ministry of Health of Ghana (Latif 2004; Commonwealth Secretariat 2011).

Similarly, other tobacco control actors and experts from Ghana participate in international conferences on tobacco, where relevant policies and information for controlling tobacco are discussed. For instance, the General Secretary of the Vision for Alternative Development (VALD) attended the premier of the Livestrong Global Cancer Summit in Dublin, Ireland, in August 2009 (Nelson 2009). The summit discussed essential strategies for controlling cancer to eliminate its burden globally. Moreover, key tobacco control actors such as Mr. Issah Ali and Mrs. Edith Wellington are members of GlobaLink, the web based tobacco control group. The membership of the GlobaLink enables the tobacco actors to have access to vital tobacco control information and literature that can be utilized in the home environment to formulate local laws or serve as means of influencing policymakers towards such objectives.

\section{Liberal-Constructivism and Tobacco Control in Ghana}

The tobacco control network is active in ensuring that there is a change of perception on the tobacco issue in Ghana to garner support for the adoption of tobacco control law. A research conducted by Dr. John Koku of the University of Ghana on tobacco farmers in the Gbefi Tornu 
and Gbefi Hoeme communities in the Volta region found that the farmers were interested in growing other crops apart from tobacco, provided that they could have some sort of micro credit (IDRC 2009). The Report noted that the farmers cited health reasons for the desire to grow alternative crops even though they found the growing of the tobacco crop to be very lucrative. The research was sponsored by the IDRC, ACS, DFID, Cancer research, UK, CRDI, and CITCR.

Mrs. Edith Wellington of the Ghana Health Service also received a similar grant to conduct research among diverse groups including Members of Parliament, policymakers, private and public media practitioners, and civil society groups in Accra (IDRC 2009). Mrs.

Wellington's research found a high level of support for the adoption of tobacco control laws including a comprehensive ban of smoking in public places (IDRC 2009). The research findings indicate that majority of Ghanaians consider tobacco consumption and its health hazards as a major problem, especially smoking among the youth, and support a ban on free samples of cigarette to minors, as well as preventing minors from purchasing or selling tobacco products.

In another development, the Chairman of the Parliamentary Select Committee on Health, Dr. Mustapha Ahmed, urged the Ministry of Health to hasten the process of introducing the draft tobacco bill to Parliament for consideration and passage into law citing the health impact of tobacco on public health as a major concern (Odoi-Larbi 2009). The call was made in his speech to mark the World No Tobacco Day in Parliament in 2009 and he indicated that it was unacceptable for nonsmokers to continue to inhale cigarette smoke. He called for immediate action to ban smoking in public places to protect nonsmokers. Odoi-Larbi (2009) maintains that the Minister for Local Government and Regional Development, who is also the Member of Parliament for Wa West Constituency, Hon. Joseph Yieleh Chireh has also called on his 
colleagues to act fast and bring a lasting solution to the problem by passing a national tobacco control law. It needs to be noted that Hon. Chireh is from the northern part of Ghana, which is one of the ecological region for the growing of tobacco. Therefore his call for the passage of tobacco bill says a lot about the impact of the tobacco control activities on the thinking of Ghanaians and policy makers. Certainly, it could be seen that majority of the public and policy makers are now convinced of the negative health impact of tobacco and are embracing the adoption of effective tobacco control legislation as a way forward to address the issue.

\section{Conclusion}

The chapter has broadly examined the efforts to control the consumption of tobacco products and its health hazards in Ghana. It was found that Ghana had tobacco manufacturing plants operating in the country until 2006 when the last manufacturing company owned by BAT relocated to Nigeria. However, the country continues to experience the activities of the tobacco industry network, in spite of the fact that there is no tobacco manufacturing physically located in the country. The activities of the industry network are often identified as the reason why the Parliament of Ghana has delayed in passing tobacco control legislation.

On the contrary, the country has also seen modest gains in the effort to control tobacco. The relocation of the BAT manufacturing plant to Nigeria is often attributed to result of the activities of the tobacco control actors. In addition, Ghana has signed and ratified the FCTC protocol and has been active in the activities of the Conference of Parties. Ghana also has several voluntary agreements to control tobacco adopted through directives of government ministries and departments, which were facilitated by the engagements of the 
transnational tobacco control actors with the Ministry of Health and domestic NGOs. Lastly, the first reading of the draft tobacco control bill has been done in the floor of Parliament and the bill has been referred to the Select Committee on Health for the final Amendments. The bill is projected to be passed into law before the end of the first term of the NDC government. The study concludes that Ghana has made some achievements with tobacco control in spite of the activities of the tobacco industry network to prevent the adoption of any tobacco control instrument. 


\section{Chapter Five}

\section{Tobacco Control Activities in Malawi}

\section{Introduction}

This chapter examines the activities undertaken by tobacco control actors to control the consumption of tobacco products and its health hazards in Malawi. Tobacco leaves farming is the main tobacco activities of Malawi and the country only had the first domestic tobacco manufacturing plant in 2009. The plant is privately owned by Nyasa Tobacco Manufacturing Company. The tobacco industry network is very active in Malawi and the members of the network are alleged to be the force behind Malawi's failure to sign and ratify the FCTC protocol up to date. In spite of this, the country engages in some tobacco control activities to curtail tobacco consumption and its associated harm. This chapter examines the political struggles between the tobacco interest groups and tobacco control actors during the attempt to adopt tobacco control law in the country. It investigates the composition of actors in both networks and also examines how the tobacco control knowledge is transferred to the country. In addition, it examine how the dissemination of relevant tobacco ideas is impacting the way the tobacco issue is understood in the country.

\section{Tobacco and the Economy of Malawi}

Malawi is one of the largest producers of tobacco leaf in sub-Saharan Africa and among the top-ten producers in the world (Makoka et al 2011). The activities of the tobacco industry and its affiliates are very strong in Malawi and the industry is often credited with preventing the country from adopting any official tobacco control legislation, in spite of the activities of the tobacco control activists. Malawi is also one of the countries where child labor on the tobacco 
farm is very high (Otanez et al 2006), and children have been exposed to nicotine levels equivalent to about 50 cigarettes a day, an amount higher than the level normally obtained from second hand smoke (Plan International 2009) ${ }^{26}$. However, there are few voluntary agreements adopted through the directives of the Ministry of Health to control tobacco but the agreements are poorly enforced.

Tobacco control activists believe that recent evidence shows a good prospect for adopting some tobacco control legislation, and they are making every effort to take advantage of the policy window before it closes. For instance, the rhetoric of politicians about tobacco control has changed and certain aspects of the FCTC measures for controlling tobacco such as providing a viable alternative to tobacco farming as means of supporting the economy have been adopted, even though the country has not signed or ratified the FCTC protocol. However, recent developments indicate that the Nyasa Tobacco Company and its political supporters are actively reversing the modest achievements (executive directives) with tobacco control attained in Malawi (Interview 2010/2011, Makoka et al 2011). The Company is vigorously advertising in both the electronic and print media (Interview 2011). An interviewee indicated that:

The Nyasa Manufacturing Company has sent the tobacco control campaign to the zero point. Since entering the market they have adopted aggressive marketing using radio and newspapers and they continue to defy all basics required for tobacco marketing. By June 17, 2011 they had erected a huge bill board at Kanengo industrial site, which is the main entrance into the Capital and has the largest industrial workforce in the country. Their banner continues to have small warnings, but deliberately mistranslated the main health warning of: smoking is hazardous to your health. The main Chichew language translation is: kusutua kumawononga moyo. However Nyasa uses the words kusuta kukhoza kuwononga moyo which translates as smoking may be hazardous to your health". Further to this is their daily campaigns where they offer their cigarettes to promotional music shows, dance competitions

\footnotetext{
${ }^{26}$ For detail of the report see http://plan-international.org/about-plan/resources/news/child-tobacco-pickerspoisoned-reveals-report and http://edition.cnn.com/2009/HEALTH/09/25/child.tobacco.picking/index.html
} 
and play loud music in rural areas to promote their brands. There is no voluntary agreement with this company and it is exploiting the situation to its advantage (Interview 2011).

\section{Tobacco Industry Network in Malawi}

The influence of the tobacco industry network in Malawi dates back to the $1800 \mathrm{~s}$ when the movement of settlers from United States and Scotland facilitated the commercial production of tobacco leaves in the country. Tobacco production has since taken a grip on the Malawian economy. Malawi has been used by the tobacco industry network to undermine the FCTC activities by arguing that the adoption of the FCTC protocol will significantly destroy her economy (Interview 2010). Malawi produces burley tobacco, fluecured tobacco, and the northern dark fired tobacco and is among the five leading producers of burley tobacco globally (Makoka et al 2011; Otanez et al 2006).

The leading tobacco producing districts in the country are Mchinji, Lasungu and Lilongwe, and these districts have large tobacco auction centers. Auction Floors are the major centers for selling tobacco leaves, mostly to the leaf buying companies, and the auction is monitored by the Tobacco Control Commission of Malawi, a government agency, which was established in 1938 to monitors the quality of the product and to also promote the tobacco industry (Interview 2010, Makoka et al 2011). Until 2009 when a domestic manufacturing company was established in the country, Malawi has always exported its tobacco leaf to other countries.

The composition of the tobacco industry network in Malawi is made up of the Ministries of Agriculture and Finance, the Ministry of Economics, certain government officials, Tobacco Association of Malawi (TAMA), Tobacco Tenants and Allied Workers Union of Malawi (TOTAWUM), tobacco leaf processing companies, auction holding 
companies, and tobacco leaf buying companies such as Universal Corporation, Alliance One International, Limbe Leaf Tobacco, and Continental Tobacco Company. The leaf buying companies export the tobacco leaves to places where companies like Phillip Morris and BAT have manufacturing plants (Interview 2010; Otanez et al 2007; Semu-Banda 2007). The tobacco leaf buying companies generate over $65 \%$ of the country's export earnings and are therefore very influential players in the Malawian tobacco business (Makoka et al 2011).

The leaf buying companies act as agents for penetration and consolidation of market for the tobacco industry network in Malawi (Cairney et al 2012). The companies market and promote tobacco use and also undermine the policies and programs of the Malawian government. Makoka et al (2011) maintain that the US tobacco-growing subsidiaries Limbe Leaf and Alliance One - are the major leaf buying companies, and they purchase over 95\% of Malawian tobacco leaves and sell them to companies like BAT or Phillip Morris. Another major player in the Malawian tobacco activities is Premium TAMA, a joint venture company between the tobacco growers association (TAMA) and the UK-based Premium Tobacco (Makoka et al 2011). Premium TAMA has an investment of over US\$22 million in the tobacco business and in October 2009, established the Kanego Tobacco Processors to process tobacco in Malawi. Although the tobacco manufacturing companies are not visibly involved in the tobacco activities, a recent report indicates that companies like BAT have a key interest in ensuring that Malawi remains a major tobacco producing economy (Makoka et al 2011) $)^{27}$.

The strong influence of the tobacco industry network, led by the tobacco leaf buying companies and their affiliates, is often alleged as the main reason why Malawi have not

\footnotetext{
${ }^{27}$ Also refer to http://www.un.org/ecosocdev/geninfo/afrec/vo118no3/183tobacco.htm.
} 
adopted any legally backed and enforced tobacco control legislation ${ }^{28}$ (Interview 2010). In other words, tobacco growing occupies a strong economic position, which causes the country to resist tobacco control, as usually the case with tobacco growing economies such as the southern states of the US. Malawi is one of the two major tobacco producing countries - the other is Zimbabwe - in sub-Saharan Africa that has neither signed nor ratified the Framework Convention on Tobacco Control (FCTC). The lobby activities of the network are mostly championed by the Ministries of Agriculture, Industries and Trade, Finance, and Economics, as well as certain government officials who have been at the forefront of resisting any attempt to adopt strict tobacco laws (Kapito 2000; Otanez et al 2006; Interview 2010). For instance, the establishment of the Nyasa Tobacco Manufacturing Company, in 2009 was championed by the Ministries of Finance and Industry (Makoka et al 2011).

Moreover, the tobacco industry network uses the politicians and influential personalities to lobby the government against the adoption of any policy that will hamper their activities and they have been able to influence the government to be very protective of the tobacco industry in Malawi (Interview 2010). In addition, the network offers funding to the Tobacco Association of Malawi (TAMA) and uses members of the Association to resist any attempt by the government to adopt tough tobacco control laws. Members of the TAMA are the local tobacco farmers and workers of the tobacco leaf proces sing companies. A participant in an interview indicated that TAMA members often receive funding and farming materials from the leaf buying companies to assist them with their farming. Additionally, Premium TAMA also receives a lot of funds from the Premium Tobacco, UK (Makoka et al 2011).

\footnotetext{
${ }^{28}$ The study ignores voluntary agreements with actors like the tobacco companies which are ineffective and mostly ignored.
} 
Although the funding to individual farmers is woefully small, it is a viable strategy used to influence the farmers to consistently lobby the Ministry of Agriculture and put pressure on the government against signing and ratifying the FCTC protocol. The majority of the farmers have argued that adopting such policies will make them unemployed, increase the level of poverty, and decrease the agricultural gross domestic product of Malawi. Nonetheless, top politicians from both the ruling party and the opposition are also known to own tobacco farms and therefore have a special interest in protecting the activities of the industry in Malawi (Makoka et al 2011). Makoka et al (2011) maintain that, John Tembo, the President of the main opposition Malawi Congress Party (MCP) is a well-known tobacco farmer and has also served as the Board Chairman for the Limbe Leaf Tobacco Company for many years. Mr. Tembo is said to be a very influential person in the Malawian Parliament, who assigns membership to the committees in the National Assembly, and therefore has a great influence on the way members vote on issues. In addition, several cabinet ministers are alleged to own tobacco farms (Interview 2011).

The network actors also use the media, sponsored researches, and affiliate groups to project tobacco as an economically important product to the Malawi, and this has been one of the key strategies used to win the support of the public and policy makers against the adoption of tobacco control laws (Otanez et al 2006). In addition, the tobacco industry network uses advertising targeting the youth to lure them to smoke (Kapito 2000; SemuBanda 2007). For instance, billboards featuring state-of-the-art digital images are normally positioned at places mostly frequented by the youth as indicated above (Interview 2010, 2011). The tobacco companies also sponsor events in which famous artists and musicians 
are used to attract and lure the youth to smoke by offering them cigarettes and other tobacco products as winning prizes or free samples (Makoka et al 2011).

The companies also offer free samples of cigarette packs to guests at hotels and use the image of famous musicians in print advertisements but include weak tobacco warning such as "smoking may be hazardous to your health" in the advertisement (Interview 2010). The tobacco control activists argue that the word "may" used in the warning is deceptive and accompanying it with a famous celebrity who is smoking might send a negative message that tobacco use is not hazardous to the person. Mostly, individual sticks of cigarette are sold to make them accessible to the poor instead of packs, even though recent legislative changes in developed countries like the $\mathrm{US}^{29}$ allows only sales of packs to consumers. In 2009, BAT (Malawi) undertook an active publicity campaign and many of the posters used in the vigorous advertising activities indicated the price of individual cigarette sticks to attract the youth and the poor. Additionally, the tobacco manufacturing and leaf buying companies, through the corporate social responsibility promotions, offer educational facilities, wells for potable water, and campaigns against child labor as means of redeeming their corporate image to the public, but at the same time they actively promote and sell their products (Otanez et al 2006, Munthali 2009).

The Youth Alliance in Social and Economic Development (YASED) reports that the largest tobacco leaf buying company in Malawi, Alliance One, has handed over a school building worth USD \$130, 000.00 to the Ministry of Education for the Kalonga Primary School (Tobacco Control in Africa 2009) ${ }^{30}$. The group also maintains that BAT donated 28

\footnotetext{
${ }^{29}$ For more on this see http://tobaccofreemalawi.blogspot.com/

${ }^{30}$ For details on the social responsibility, visit http://blogsofbainbridge.typepad.com/africa/2010/01/malawi-lawsuitagainst-a-tobacco-control-advocate.html
} 
bicycles to the Ministry of Agriculture in 2009. Limbe Leaf Buying Company has also donated an equivalent of US \$17,142.00 to the Church of Central Africa Presbyterian (CCAP) for its reforestation program in 2009 (Makoka et al 2011; Malawi Nation; Malawi Daily Times). Tobacco control activists argue that the donations should be considered as atonement for some of the damages caused to Malawians by the tobacco industry network due to their activities in the country. For instance, they argue that the donation towards reforestation is repayment for the lost forest in the tobacco growing areas (Makoka et al 2011).

The actors of the tobacco industry network also use lawsuits and the threat of lawsuits to scare and silence tobacco control activists. A case in point is where a leading tobacco control activist, John Kapito, was sued for libel and defamation by the Nyasa Tobacco Manufacturing Company for commenting on the deceptive advertisements by the company, in spite of the Ministry of Health directive that has banned tobacco advertisement on the radio and television (Munthali 2009). The tobacco control activists allege that these reasons account for why the Malawian government has not signed or ratified the FCTC, forcing the Ministry of health to the use directives to control tobacco instead of tobacco control legislation that could be properly enforced with the full force of the law (Interview 2011). A respondent indicated that:

the absence of a strong anti-tobacco lobby, and the opposite being true about the industry, sees Malawi's position as being industry driven. The last position after the Uruguay COP meeting was to sign by January 2011, but it seems the industry wants to play a wait and see approach. They use Malawi as the last post for making their case about the impact of poverty and employment should the FCTC continue introducing what they consider as stifling measures. Should Malawi sign the FCTC, I am sure the industry will create another excuse not to force the implementation. However, just by signing, it would be a great opportunity for tobacco control to take up the campaign and force government to follow the FCTC to the letter (Interview 2011). 


\section{Tobacco Control Network Activities in Malawi}

The tobacco control network is very active in Malawi and the actors engage in activities aimed at curtailing the influence of the tobacco industry network and the hazards associated with the consumption of tobacco products. Like Ghana and many developing countries, the Ministry of Health of Malawi is the main governmental agency that promotes tobacco control, because of the health hazards associated with tobacco consumption. The Ministry works with other agencies and anti-tobacco advocacy network actors like Smoke Free Malawi, operated by the Youth Alliance Social and Economic Development (YASED), and the Consumers Association of Malawi (CAMA), Centre for Social Concern, Cancer Registry of Malawi, Health Journalists Network, Journalists Union of Malawi, Medical Doctors Association of Malawi, National Youth Council of Malawi, World Health Organization Communication Office, Drug Fight Malawi, Link for Education and Governance, lawyers, researchers, and individuals with the passion to control the activities of the tobacco industry and the negative hazards of tobacco in Malawi.

The actors have conviction that a broad and sustained educational program that targets the public - including government officials, women, youth, children and tobacco farmers - will help to galvanize support for tobacco control in Malawi (Makoka et al 2011; Interview 2011). Malawi has a few voluntary tobacco control regulations, which were adopted through the directives of the Ministry of Health. However, the regulations are not properly enforced because they were not legislated by the Parliament of Malawi, which is the official law making body. The voluntary tobacco control agreements include a smoking ban in some public and private places, including domestic flights of Air Malawi, airports, and fuel stations adopted since 1999. 
In addition, a directive to ban radio and television advertisement and also to require a health warning to be carried in print advertisements has been in place since 2005. Moreover, the Ministry of Health also issued a similar directive to ban tobacco sponsorship and promotional activities from the sports and entertainment industries in the same year. The Minister of Finance in 2008/2009 budget statement increased the excise tax on tobacco through the introduction of a system of tax stamps (Hajat and Milanzi, 2008). The Minister also adopted an additional duty system that was based on the quantity of cigarettes imported or produced in the country instead of the value. Hajat and Milanzi, (2008:14) maintain that the duty introduced were new flat rates ranging from the equivalent of $\$ 18$ per thousand sticks for hinge-lid packets and \$12 per thousand sticks for soft-lid packet of cigarettes.

The intention of the new excise tax was to control smuggling and promote Malawian grown tobacco, as cigarettes that contain at least $70 \%$ of Malawian tobacco attract a much reduced tax rate (Makoka et al 2011). The rates for cigarettes with Malawian tobacco were equivalent of $\$ 9$ per thousand sticks for hinge-lid packets and $\$ 6$ per thousand sticks for soft- lid packet, which was much lower than the rate for those imported into the country. The hinged-lid packet of cigarettes comprise an inner package defined by a group of cigarettes wrapped in a sheet of foil inner wrapping and a rigid outer package housing the inner package that helps to prevent loss of essential ingredient of the cigarette or becoming soft once the lid is open. Therefore, the hinge-lid cigarettes tend to be more expensive than the soft-lid packet, which are basically the cigarettes in one layer soft box (Interview 2011).

The Ministry of Health and the domestic nongovernmental organizations in conjunction with interested individuals continue exert pressure on the government of Malawi to sign and ratify the FCTC protocol and also adopt tobacco control laws that are 
legally binding and effectively enforced by highlighting on the negative health effects of the tobacco products. For instance, the Youth Alliance in Social and Economic Development (YASED), which operates the Smoke Free Malawi project, has been campaigning and highlighting the negative effects of tobacco and smoking on public health (Munthali 2009). The group has instituted a two-year public campaign to mobilize support aimed at putting pressure on the government to sign and ratify the FCTC protocol and also to ban public smoking in the entire country (Makoka et al 2011, Interview 2010). A case in point is a program jointly established by YASED and the National Youth Council of Malawi (NYC) and the Centre for Agriculture Research and Development (CARD) of Bunda College, Germany, to educate young people on the dangers of smoking (Munthali, 2009).

The tobacco control actors believe that there is the window of opportunity for adopting some tobacco policies if they persist with their activities and frame the issue as a public health issue (Interview 2010, 2011). An interviewee indicated that their belief: is premised on the fact that increased public awareness on the dangers of tobacco will shift the focus from the political and economic debate on the role of tobacco that the government and its tobacco allies have exploited by using fear to quash attempts to create awareness on dangers of tobacco. A good strategy of using it as a public health issue creates personal awareness as individuals will reflect on causes of death within their own set up, which would easily counter the political-economic rhetoric that has been used to silence tobacco control efforts. With high awareness levels, very few would voluntarily wish to grow, smoke or produce tobacco and government will be under pressure to identify alternatives to the economy with urgency (Interview 2011)

The anti-tobacco groups consider the education of the public on the negative hazards caused by the tobacco industry as a strategy to neutralize the strong activities of the tobacco industry in Malawi. To that effect, the members of the Smoke Free Malawi have been highlighting a report by PLAN Malawi indicating that children working in the tobacco industry are exposed to high levels of nicotine. PLAN Malawi is the Malawi branch of 
PLAN International, a nongovernmental child development organization that works in over 48 countries in Africa, Asia and American continents to promote child rights and lift millions of children out of poverty (Interview 2011).

Smoke Free Malawi members are using the report to pressure the government to sign and ratify the FCTC protocol and also adopt appropriate tobacco laws to protect the health of children and other groups exposed to the dangers of nicotine (Interview 2010). The group uses the report to gain support of the public for tobacco control by stressing that if nothing is done by the government, Malawi will soon witness an epidemic of tobacco related diseases such as cancer. An interviewee further noted that highlighting the issues will help to put more pressure on the Malawian government from other powerful actors and international organizations such as the Commonwealth and WHO.

The tobacco control activists also organize protest marches against the activities of the tobacco industry as part of achieving their objectives. Munthali (2009) reports of the peaceful demonstration organized by civil society groups, cancer groups, against the meeting of ITGA held in Salima, Malawi, from May $3^{\text {rd }}$ to $6^{\text {th }} 2009$, during which the chief executive of ITGA was in attendance. The protest activities, included press conferences on the harmful effects of tobacco on the people and economy of Malawi, demonstration of posters from the airport through the major routes where the delegates to the meeting travelled, submission of petitions to the delegates, and a radio broadcast of public debate on tobacco, which was aired on two major radio stations in the city.

Many of the activists of Malawi have been invited to participate in regional and international conferences (Interview 2010). The Ghana News Agency on February 18, 2009 published an article on a tobacco control training program organized by the Africa Tobacco 
Control Regional Initiative (ATCRI). The meeting was meant to equip members with skills on policy and advocacy to help change policy and to train them in epidemiology necessary to make a difference in their respective countries. Participants from Malawi took part in the training program. In addition to regional conferences, the tobacco control actors in Malawi also benefit from informational resources through their membership of the GlobaLink, managed by the International Union against Cancer (Interview 2010/2011).

John Kapito, a tobacco control activist in Malawi, is listed as a beneficiary of a $\$ 20,000$ two year grant, by the American Cancer Society (ACS), the International Union against Cancer (UICC), and the Cancer Research UK to pressure the government of Malawi to sign and ratify the FCTC and also adopt relevant domestic tobacco control laws. A status report by John Kapito indicates that the activities carried out with the grant have generated some consensus among the key stakeholders for the government to sign and ratify the FCTC (ACS, UICC and Cancer Research UK, 2004). The report further indicates that the President of Malawi authorized the Ministry of Foreign Affairs to sign the FCTC, but the 2004 presidential election and the absence of cabinet at that time delayed the signing of the protocol, and Malawi has not signed the protocol to date. In addition, Mr. Deusdedit Peter Kafere of the University of Malawi also got a grant from the IDRC to undertake a research to determine the critical determinants for tobacco control in the country in 2008 (IDRC 2008).

The collaboration between the international NGOs, IGOs and local actors has compelled the government to adopt laws that indirectly promote the spirit of tobacco control such as the measures for strengthening the child labor laws, which has been reauthorized continuously (Interview 2010). Malawi passed a labor law in 2000 to prevent children from working on the farms and has since been periodically strengthening their labor laws to prevent 
the exposure of children to nicotine by working on the tobacco farms ${ }^{31}$. Recently, over 300 labor officers have been trained by the Ministry of Labor and Vocational Training (MOLVT) and given the responsibilities to monitor, inspect and report child labor issues and also to enforce child labor laws, together with the police. The Ministry and other organizations have organized youth committees in the villages and towns and tasked them to report issues of child labor for prosecution. There have been significant convictions, in which violators were fined up to the equivalent of US $\$ 200$ or a maximum of 5 years in prison as required by the Employment Act of 2000. The Ministry of Gender, Child Welfare, and Community Services has been tasked to formulate policy on childcare and protection. The Ministry, together with the Department of Social Welfare, offers assistance to children on the street to avoid exploitation by the tobacco farmers.

In view the high level of the use of child labor in tobacco farms, a core objective of antitobacco advocacy network members was to promote the adoption of laws that will protect the children from the exposure to the nicotine either through second hand smoke or working on the farms. Together with other NGOs and human rights organization, pressure was mounted on the government, which resulted in the adoption of the child labor law and it has been strengthened since it was first adopted. The most recent effort in strengthening the laws was in 2010, in which the minimum age of labor was raised from 14 years to18 years (Interview 2010), and the laws are properly enforced because it was legislated by the National Parliament. In addition, the government adopted a Free Primary Education policy in 2002 to decrease child labor by providing stability for children to remain in schools (Kayange 2003) ${ }^{32}$. Kayange (2003)

\footnotetext{
${ }^{31}$ For details see http://www.cridoc.globalmarch.de/child_labour.html

32 See Kayange, George 2003 “Child Labor Situation in Malawi: An Overview” Child Rights Information and Document Center Retrieved from website: http://www.cridoc.globalmarch.de/child_labour.html on 4/15/2010
} 
maintains that the government has continuously allocated substantial budgetary resources to the Ministry of Education to run this program.

According to some tobacco control activists, the adoption and strengthening of the child labor laws to a large extent indirectly achieves the objective of curtailing the exposure of children to high levels of nicotine despite the fact that a direct tobacco control law on second hand smoke has not been adopted. They argue that the policy might seem symbolic, but it is a significant success chalked up by the tobacco control network's campaign and activities because the tobacco leaf buying companies are forced to abandon the use of child labor that makes tobacco leaves and farming in Malawi cheap and attractive.

Consequently, many farmers are losing interest in growing tobacco products and seeking alternative crops to grow because it is expensive to grow tobacco without the cheap or free labor from the children (Interview 2010). However, the tobacco interest groups and farmers are not attacking the government for the adoption of the policy because the policy is considered a labor laws and not a direct tobacco control measure (Interview 2010, 2011). There is also a significant change in the rhetoric of government officials about the need to ratify the FCTC as some officials have indicated that the country will ratify the FCTC protocol, in spite of the fact that it has not happened yet (Interview 2010).

\section{Knowledge Transfer and Tobacco Control in Malawi}

The knowledge transfer agents use several means including the dissemination of new ideas and incentives to engage governmental institutions such as the Ministry of Health to transfer tobacco control knowledge for adoption into domestic law in Malawi. The officials of government and domestic NGOs and other actors also gained knowledge on new 
strategies for controlling tobacco through participation in international conferences like the Framework Convention Alliance, WHO, Commonwealth, and the World Conference on Tobacco or Health, and meetings of the COPs (Interview 2010). In addition, the members get to learn of the best practices for controlling tobacco that has been applied somewhere through GlobaLink, which is serving as the biggest online resource for tobacco control knowledge transfer (Mamudu 2005, 2008; Interview 2011).

Moreover, knowledge is also transferred through other means of contacts and agreement with the government of the receiving country. For instance, the government of Malawi has adopted measures to diversify the economy through direct communication with the US. The government signed a memorandum of understanding (MOU) with the US government under the United States African Development Foundation (USADF) in April 2009 to promote development, diversify the economy, and increase participation of groups in trade and investment activities ${ }^{33}$. Among the core groups who received funding were the Roots and Tubers Enterprises Farmer Group (CMRTE) to help with the growing of roots and tuber crops in order to reduce the dependence of the economy on tobacco.

The U.S is known for the expertise in supporting its economy with different sources of revenue. Through the provision of financial incentives, the U.S government has been able to transfer their knowledge on diversifying the economy to Malawi to make the Malawian economy less dependent on one product. The assistance to Malawi could be classified as a form of indirect coercive knowledge transfer, whereby IGOs and governments use incentives to pressure the adoption of certain policy tools but fails to offer the adopting country the option of selecting

\footnotetext{
${ }^{33}$ For details on the MOU, visit http://www.adf.gov/USADFFirstGrantSignedinMalawi.htm
} 
from different policy tools that they have the capability and incentives to learn about the outcome of their actions (Schneider and Ingram 1988).

A participant in the interview indicated that the initiative was taken because the U.S provided funding for the program and Malawi was asked to use the money specifically for that project. Adopting policies to diversify the economy is a major measure recommended by the FCTC and it is important for tobacco control in Malawi because of the high level of dependence of the economy on tobacco. The government was compelled to adopt the measure because tobacco control activists collaborated with other interested organizations to reframe the tobacco issue as an issue of poverty of the farmers because the farmers lack an alternative means of livelihood or other economic activities than tobacco growing (Interview 2010).

A clear observation is that whereas the intention of the countries transferring knowledge and tactics prefer to get the policy makers of receiving countries to adopt them into domestic policies, the receiving countries have conditions that determine which tactics or ideas get adopted into domestic policy. For Malawi, the litmus test for a particular knowledge or tactics to be adopted into domestic policy is political economy - the impact of the policy on the Malawian economy (Interview 2010). Therefore, any knowledge or tactic that was perceived to have a negative or adverse effect on the economy was less likely to be supported by the public or to be adopted into domestic policy by the policy makers. The adoption of this measure to diversify the types of crops that farmers grow was a safe zone for the policymakers because it intended to provide alternative farming activity to the farmers without directly calling for an end to tobacco leaf growing.

Measures like this is the reason why the tobacco companies and their affiliates have been projecting tobacco as the major blood vessel for the survival of the economy while the 
anti-tobacco advocacy network actors have also been intensifying their activities to debunk the argument of the former as baseless. Both of these networks use sponsored researchers, the media, and other means of communication to get their message to policy makers and the public. However, the tobacco companies seem to enjoy more advantage over their counterparts on this propaganda game because of their resources and established position. So far, the anti-tobacco advocacy network has reframed the tobacco issue as that of poverty and public health, which they consider essential to expand on the little success that they have chalked up this far.

\section{Liberal-Constructivism and Tobacco Control in Malawi}

Malawi also shows evidence of the struggles to control the minds of the people on the tobacco issue. The findings in Malawi suggest that the tobacco control activities are having influence on how the tobacco issue is perceived or understood. For instance, a recent research survey, sponsored by the Bill and Melinda Gates Foundation and the Canadian International Development Research Center found that $80 \%$ of non-smokers and over $50 \%$ of smokers in Malawi would support a tobacco advertising ban in Malawi (Makoka et al $2011)^{34}$. Also, a member of the Malawian delegation to the 2007 Commonwealth Health Ministers Meeting (CHMM) indicated that effective tobacco control is the only means through which the rising tuberculosis (TB) rates in Malawi can be controlled (Commonwealth Secretariat 2007). Recent research by the WHO found direct

\footnotetext{
${ }^{34}$ See the details of this report at https://idl-bnc.idrc.ca/dspace/bitstream/123456789/42362/1/129788.pdf
} 
epidemiological evidence that links smoking to TB infection and also for the development of pulmonary $\mathrm{TB}^{35}$.

In addition, the leader of the Malawian delegation to FCTC in 2003 indicated that the FCTC was a health convention and not a tobacco treaty. Moreover, a Principal Secretary of the Ministry of Agriculture indicated in January 2009 that Malawi will sign and ratify the FCTC (Tobacco Control in Africa 2009). He argues that ratifying the Convention will enable Malawi to have a voice at the meetings of the FCTC to speak in favor of tobacco. In spite of the fact that the government has not yet signed and ratified the protocol, the tobacco control activists see the pronouncement and change in the position of the Malawian government as a major milestone because Malawi was one of the countries used by the tobacco industry network to abandon the FCTC when it was established (Interview 2010). The government had indicated consistently that it would not ratify the FCTC in the past and activists believe that once the country signs and ratify the protocol, it could be used to put pressure on the government to adopt tobacco control legislation.

As earlier indicated, the campaign for protecting children against the dangerous effects of nicotine through working on the tobacco farms ${ }^{36}$ and second hand smoke has compelled a joint effort of government, tobacco leaf buying companies, and affiliated NGOs to adopt measures that will prevent the exposure to nicotine. The tobacco companies and affiliates have been compelled to establish a foundation known as Eliminating Child Labor in Tobacco Growing (ECLT) ${ }^{37}$ to enforce the prevention of child labor on the tobacco farms

\footnotetext{
${ }^{35}$ For details on the research finding: http://www.searo.who.int/en/Section10/Section2097/Section2106_10682.htm

${ }^{36}$ An interview participant indicated that research has shown that the amount of nicotine absorbed into the body a day through collecting the tobacco leaves is about the equivalent of 50 cigarettes and more than that obtained through second hand smoke.

${ }^{37}$ For more on the activities of the ECLT, see http://www.eclt.org/site/wp-content/uploads/2010/02/ECLT-inMalawi-Quick-Facts.pdf
} 
(Otanez et al 2006). Even though the government has not adopted a direct smoke free environmental law, the strong child labor laws and measures will curtail the exposure of children to the dangerous effects of nicotine as a result of working on the tobacco farms. However, Otanez et al (2006) maintain that the tobacco companies and their affiliates are using the ECLT project as a cover up on the real negative effect of tobacco on public health and also to boost their corporate image. In addition, the public mounts pressure on the government for the adoption of policies to diversify the economy to relieve it from the overreliance on only tobacco products. A tobacco control activist in an interview indicated that the recent support among the public for an advertising ban and diversifying the economy is an indication that there is a change in the understanding of tobacco from its economic importance to its harmful health hazards.

The activist indicates that people are paying more attention to the negative health hazards of tobacco preached by the tobacco control activists than the economic importance projected by the tobacco companies. In spite of the fact that the government has been dragging its feet to adopt a tobacco law because of the influence and resistance by the tobacco companies, recent pronouncements and activities show a growing interest of the government for the adoption of some tobacco laws to protect public health.

\section{Conclusion}

The analysis above shows that effort to control tobacco in Malawi is stalled by the active tobacco growing activities, which has assumed a strong economic position in Malawi. It illustrates the struggles between the tobacco control activists and tobacco industry network in the effort to win the mind of the public about the tobacco issue. The tobacco control activists (the 
anti-tobacco advocacy network) have framed the tobacco issue as a public health issue to mount pressure on the policy makers in speeding up the control of tobacco and its health hazards. However, the tobacco companies and affiliates (pro-tobacco advocacy network) have also been using all kinds of strategies to prevent the adoption of tobacco measures, which have the tendency of decreasing their activities. The tobacco industry network continues to project the economic importance of tobacco to the development of the countries.

The activities of the tobacco companies are supported by government officials and influential politicians, many of who have tobacco farms and therefore try to prevent the adoption of effective tobacco control laws that will destroy their investments. However, Malawi has few tobacco control voluntary agreements instituted through the directives of the Ministry of Health but the directives are not properly enforced. Malawi has also adopted laws that indirectly fulfill the spirit of the tobacco control by protecting children from exposure to nicotine. The country also provides the farmers with alternative crops to grow to reduce the dependence on tobacco leaves. However, a privately owned tobacco manufacturing company established in the country in 2009 is openly ignoring the directives on the advertisement ban. The tobacco control activists believes that framing the tobacco issue as a public health issue will help to win support for the adoption of effective tobacco control laws. They cite a recent survey showing that the public favors the adoption of certain tobacco control measures to protect public health as evidence. 


\section{Chapter Six}

\section{Evaluation and Conclusion}

\section{Introduction}

The central topic of this study is to examine the tobacco control policies adopted in Malawi and Ghana focusing on the politics between the tobacco control and the tobacco industry networks during the process of adopting tobacco control laws. Ghana and Malawi are Commonwealth member countries in the sub-Saharan African with similar political institutions, policy culture, histories of tobacco prevalence, and are also at the same stage of the tobacco epidemics (Lopez et al 1994; Studlar 2002). Both countries have agrarian economies and rely on or have at a certain point relied on tobacco products as a major contributor to their gross domestic product (GDP). Each of the countries also has some tobacco control activities going on because of the influence of international dynamics, the expansion of the activities of the tobacco companies, effort to control illicit trade in tobacco, and the desire to prevent the harm associated with tobacco consumption.

However, the trajectory and extent of tobacco production and control efforts in the two countries have been different for varied reasons. The response to the efforts to control tobacco by the two countries has yielded different results, which necessitate an investigation into the factors that account for the similarities and differences in their policy behavior (Studlar 2002). The countries have not adopted any formal tobacco control legislation that is effectively enforced but have used administrative directives and taxation to control tobacco production and consumption. However, the taxation and the directives have been used for different purposes in the two countries. For instance, each of the countries use taxation to raise revenue but Malawi 
also uses the taxation to promote its tobacco while Ghana uses it to control tobacco smoking (Interviews 2011).

Ghana has made modest gains in sustaining the voluntary agreements instituted through various ministerial directives and has a tobacco bill that is currently before the Parliamentary Select Committee on Health for the final amendments before it is enacted into law. But the contrary has been the case with Malawi, where the minimal gains achieved in the effort to control tobacco are deteriorating partly due to the level of dependence of the Malawian economy on tobacco products. In spite of this, tobacco control activists believe there is a window of opportunity to improve upon the minimal gains that needs to be taken advantage of by framing the tobacco as a public health issue.

A combination of three theories comprising the multi-level knowledge transfer theory, the global advocacy network theory, and the liberal - constructivist theory, was used to examine the process of tobacco activities in the two countries. This chapter specifically analyzes the validity of these theories in explaining the politics underlying the adoption of domestic tobacco control policies in the two countries. The data so far indicates that all the theories collectively help in understanding the politics underlying the adoption of tobacco control policies in Ghana and Malawi. On an individual level, the global advocacy network theory to a large extent helps to explain the politics underlying the adoption of tobacco control policies in Ghana and Malawi better. However, it is also important to stress that the theory alone does not provide a complete understanding of the politics and a complete picture of the politics is obtained when the theory is complemented with the knowledge transfer and liberal-constructivist approaches. While the global advocacy network theory helps to identify the actors who are promoting their interest as tobacco control ideas for adoption into laws globally, the multi-level policy transfer approaches 
also present an understanding of how the actors engage the bureaucratic institutions mainly the Ministry of Health to influence it to adopt the tobacco control ideas. Lastly, the liberal constructivist theory provides explanation on why the countries being contacted decide to adopt or not to adopt the tobacco control ideas being transferred to them.

By way of presenting the findings, this chapter comparatively discusses the voluntary tobacco control agreements adopted through the directives of government ministries and agencies spearheaded by the Ministry of Health in Ghana and Malawi as well as measures to consolidate the tobacco control efforts by adopting legislation that is effectively enforced. In addition, the research questions posed for the study are examined using the data collected from

the responses from the interviews and secondary documents. It also analyzes the motives behind the adoption of the voluntary agreements and tax measures to understand how a similar measure could be adopted for a different purpose in each of the countries. The chapter also examines the action of the tobacco industry network and explains how the structure of tobacco industry network in each country impacts tobacco control activities. Lastly, it presents the impact of tobacco control in the developing countries, the shortcomings of the study, and identifies the areas for future research.

\section{Tobacco Control Coalitions in Ghana and Malawi}

Ghana and Malawi share similar experiences in terms of the interest groups and actors who play vital roles in influencing policy making in tobacco control of the countries. As former colonies of Britain, both countries fall prey to the transnational tobacco companies (TTC) especially the British American Tobacco (BAT) and Phillips Morris, which has established strong manufacturing, trading relations, and/or production bases in 
almost all former colonies of Britain (Latif 2004). The British American Tobacco Company, other TTC, and their affiliates such as the ITGA have presented themselves as natural allies of tobacco growers and retailers, and are actively working with the local businesses, farmers and organizations built around the value of the tobacco industry to prevent the adoption of stricter domestic tobacco laws in almost all the Commonwealth countries (Latif 2004).

\section{Global Advocacy Network and Tobacco Industry Network}

Ghana and Malawi experience the activities of the tobacco industry network in different ways. In Ghana, the tobacco industry network activities have been spearheaded by tobacco companies like British American Tobacco, Embassy, distributors, and importers of cigarettes into the country. Since 2006, there has been no tobacco manufacturing plant visibly located in Ghana because of unfavorable environmental conditions for production. However, the tobacco interest groups are still influential and they are actively lobbying policymakers against the adoption of legally backed tobacco legislation that will hamper their business.

Some tobacco control activists maintain that the visible presence of the manufacturing plant in Ghana, including a state-owned plant at a point in time, was a blessing in disguise as it made it easy for tobacco control actors to demonize and project the companies as the bad guys who were promoting their business and profit ventures at the expense of public health. An alternative explanation is the fact that tobacco production did not emerge as one of the major contributors to the GDP and foreign earnings of Ghana may has made it easier for certain voluntary agreements to be adopted. Ghana has relied on different agricultural products as major contributors to the GDP and the cocoa crop farming has been a major source of employment and export commodity. This may have made it easier for Ghana to promote the adoption of tobacco 
control instrument, unlike Malawi where the tobacco product is both the major source of employment and contributor of foreign earnings. In spite of this, the tobacco network is alleged to have forestalled the passage of the comprehensive tobacco legislation by the Parliament of both countries. Instead the network has entered into voluntary agreement with the Ministry of Health to promote the adoption of administrative directives for controlling the consumption of tobacco in both countries. The voluntary agreements have been considered as weak laws for controlling tobacco in most western countries (Studlar 2004). In addition, tobacco control activists believe that the visit of the President of British American Tobacco and his private discussion with President Kufour, the former President of the Republic, resulted in the failure of the cabinet to introduce the draft tobacco bill to Parliament for the passage into law.

In Malawi, the tobacco leaf processing and buying companies allegedly collaborate with certain government ministries and tobacco growers and workers associations in the country to resist the adoption of any tobacco control legislation. Malawi's tobacco activities mainly involve the growing of tobacco leaf for export, and also as raw material for a tobacco manufacturing plant recently established in the country. The tobacco sector offers employment to over $50 \%$ of Malawians and contributes a high percentage to the foreign earnings and the gross domestic products of the country. Therefore, as is usual with tobacco growing economies (Cairney et al 2012), the Malawian policymakers are not friendly to tobacco control ideas. Key policymakers and politicians are active members of the coalition resisting the adoption of effective tobacco control measures by the government to protect their investments - tobacco farms.

The extent of dependence of the Malawian economy on tobacco products enabled the international tobacco growers association to use Malawian tobacco leaf growers/farmers to lobby against the adoption the FCTC measures on grounds that it will destroy their economy. The 
tobacco leaf buying companies through their corporate responsibility programs offer developmental projects and assistance to communities to maintain a favorable image in spite of the fact they continuously promote activities of the tobacco industry network. Malawi has not signed or ratified the FCTC protocol, and the few voluntary agreements on tobacco control measures instituted by the directives of the Ministry of Health are deteriorating in enforcement following the establishment of the Nyasa Tobacco Manufacturing Company in 2009 because the company has no voluntary agreement with the Ministry of Health.

\section{Global Advocacy Network and Tobacco Control Network}

The Ministry of Health of Ghana and Malawi, which champions tobacco control activities in each country, collaborates with domestic and international tobacco control organizations to promote tobacco control activities. Some of the key partners in pushing for the adoption of policies to protect international public health are the World Health Organization and the Commonwealth. The World Health Organization champions the adoption of policies to promote global public health. Additionally, it is the umbrella body housing the FCTC and actively champions tobacco control. The FCTC was established as an international regulatory body to protect the drastic challenge posed by the tobacco epidemic. Therefore, the WHO is actively involved with tobacco control activities and has been at the fore front in the promotion of tobacco control policies to prevent the health hazards associated with tobacco.

The WHO, through the Tobacco Free Initiative (TFI), coordinates global response to control tobacco use and also facilitates the implementation of the FCTC protocol, especially with capacity building at the country level (Interview 2011). The TFI organizes the Annual No Tobacco Day celebrations, which are used mainly to educate the public on the dangers associated 
with tobacco consumption. In addition, the Health Ministers of member countries through the Commonwealth Health Ministers Conference deliberate on key public health policy issues, which impact the policy adoption in their respective countries (Krishnan et al 2003, Commonwealth Secretariat 2007, 2011). Therefore, as member countries of both organizations, Ghana and Malawi are influenced by the activities and decision of the WHO and the Commonwealth on public health, in which tobacco control looms large.

International nongovernmental and governmental organizations also play influential role in the tobacco control activities of the two countries. For instance, the Bill and Melinda Gates' Foundation offers sponsorship to the African Tobacco Control Consortium administered by the American Cancer Society (Becker 2010, Interview 2011). The Consortium coordinates and leads evidence-based approaches to control tobacco consumption and its health hazards in the region. The Foundation through the RITC/IDRC supports the African Tobacco Situational Analysis project, which helps develop the capacity of researchers to gather, synthesize and analyze relevant tobacco control data (IDRC 2009). Ghana and Malawi were among the twelve countries selected as case studies to analyze the broader social, economic, and political tobacco activities to identify policy priorities and interventions (IDRC 2009). The Consortium found that some of the factors that inhibit the progress of tobacco control in sub-Saharan Africa are inadequate funding for tobacco control, the lack of political will, and the present of a weak civil society that lacks the needed capacity to influence policy change (Interview 2011).

The Bloomberg Foundation and Initiative also offered a grant to the Vision for Alternative Development, a nongovernmental organization in Ghana, to undertake a consolidated campaign for the implementation of FCTC Article 11 (pictorial warning) and also to advocate for the adoption of a strong national tobacco control bill in Ghana (Interview 2010). In addition, Ms. 
Edith Wellington, the contact person for tobacco control at the Ghana Health Service, and Mr. Deusdedit Peter Kafere benefitted from IDRC/RITC Africa Tobacco Situational Analysis Development grant for undertaking research aimed at understanding the determinants of effective tobacco control measures in the region (IDRC 2008). In Malawi, Mr. John Kapito, a tobacco control activist, also benefitted from a grant meant for putting pressure on the government of Malawi to sign, ratify and adopt relevant tobacco control laws offered by the ACS, UICC and the Cancer Research, UK (ACS et al 2004).

Additionally, the Africa Tobacco Control Alliance received funding from the Framework Convention Alliance, the International Development Research Center of Canada, and the Norwegian Cancer Society to educate policymakers on the link between tobacco control and poverty alleviation and also to lobby the policymakers for the inclusion of tobacco control in their poverty alleviation programs (ATCA 2009). The Global Youth Tobacco Survey has also conducted surveys in both countries to provide data on the prevalence of tobacco use among the youth to help with the tobacco control strategies. The assistance, collaborations, and funding have enabled the regional and domestic tobacco control organizations in Africa and the countries, respectively, to organize campaigns, capacity building activities and educational programs aimed at facilitating the adoption of comprehensive domestic tobacco control policies that are effectively enforced. Even though neither of the two countries has gotten to that stage yet, both of them have some voluntary tobacco control measures in place and Ghana is closer to passing comprehensive tobacco control legislation. So far, the impact of the tobacco control voluntary agreements in Ghana and Malawi have been different, and the purpose of the adoption of some of the measures were different in each of the countries. 
Table 6.1: $\quad$ Status of Policies and Progress towards Selected Demand Reduction Tobacco Control Policies/Measures

\begin{tabular}{|l|l|l|l|l|l|l|}
\hline Country & Monitor & $\begin{array}{l}\text { Protect from } \\
\text { tobacco smoke }\end{array}$ & $\begin{array}{l}\text { Offer help } \\
\text { to quit } \\
\text { tobacco use }\end{array}$ & $\begin{array}{l}\text { Warn about the } \\
\text { dangers of } \\
\text { tobacco }\end{array}$ & $\begin{array}{l}\text { Enforce bans } \\
\text { on tobacco } \\
\text { advertising }\end{array}$ & $\begin{array}{l}\text { Raise } \\
\text { taxes on } \\
\text { tobacco }\end{array}$ \\
\hline Ghana & 3 & 3 & 4 & 5 & 4 & 4 \\
\hline Malawi & 3 & 2 & 2 & 2 & 3 & 4 \\
\hline
\end{tabular}

\section{Voluntary Agreements (Tobacco Control Instruments) Adopted in Ghana and Malawi}

The analysis of data reviews that Ghana and Malawi are at different status with regard to their progress with adopting certain tobacco control measures (refer to Table 6.1). Ghana is ahead of Malawi in the adoption of certain demand reduction control measures (WHO 2011). The status of the tobacco control policies and progress for certain demand reduction measures for Ghana and Malawi were examined using a scale and data from a previous study by the World Health Organization. The World Health Organization indicates that the estimation was made based on survey and other data reported by the countries, which were scored according to indicators developed by the WHO (WHO 2011). This study used the scale to measure the progress of some tobacco control instruments of Ghana and Malawi based on data collected through surveys and analysis of secondary materials. Ghana ranked higher than Malawi in the measurement indicators for all the instruments, except that of the monitoring measure.

\footnotetext{
${ }^{38}$ An additional scale was added to this category to take care of Ghana's peculiar situation. In addition, Ghana meets all the characteristics of the $* *$ (specific health warnings mandated), except the language one where the warnings are written in only English, the official language of business and instruction.

${ }^{39}$ Refer to the appendix for the keys to the scale used
} 


\section{Monitoring}

Both Ghana and Malawi ranked in the third category on the implementation of the FCTC monitoring measure (WHO 2011). The countries had data that were from 2003 or recent and a survey sample that represented the national population. The status of the policies was examined using a four scale ranking that looks at how representative and current the data were. Both countries had some sort of a survey to monitor the prevalence of tobacco use among the people. The GYTS tobacco surveillance study was conducted in Ghana in 2000 and 2005 to assess tobacco use among the youth. In addition, a national demographic and health survey to determine tobacco prevalence among adult were conducted in 2003 and 2008. Malawi also had the GYTS survey in 2006 to assess the prevalence of tobacco use among the youth, and a national survey to determine tobacco prevalence among adults were conducted by the World Health Organization in 2003 and 2005.

\section{Protection against secondhand smoke or "passive smoking"}

The indicator for this measure was based on the number of public places that are completely smoke-free (WHO 2011). Both countries have some voluntary tobacco control agreements on smoke free environments in certain public areas and Ghana falls within the category three of the scale while Malawi is in the category two. Ghana has voluntary tobacco agreements that prohibit smoking in all government buildings, Ministry of Health facilities, port and harbor, stadia, airport, domestic and international airlines, public and certain private transport as well as agreement prohibiting smoking in all domestic and international airlines. The national tobacco steering committee and the Ghana Tourist Board (GTB), the agency in charge of tourism has been able to reach an agreement with the hospitality industry - restaurants and hotels - to designate certain areas as smoke free. The Ministry of Education also prohibits 
smoking by school children and teachers within certain parameters of the school and it administers severe punishment to those caught violating the directives.

Additionally, the Ministry of Education of Ghana in 2009 established a School Health Education Policy that encourages child friendly school environment and healthy lifestyles and prohibits tobacco use as well as emphasize counseling services for students who want to quit smoking (MOH, GHS \& FCTC 2010). The directives for smoke free environments in Ghana started in the 1980s when the government administratively restricted smoking in all government facilities, offices, and public places such as cinema centers and restaurants, and has continued with the active involvement of other ministries that have issued similar administrative directives. Recently, the Ministry of Health has issued another directive to have no smoking signs to be mounted on all the premises of all health facilities (Ghana News Agency, June 4, 2011). Malawi also has voluntary tobacco control agreement that prevents smoking in domestic flights of Air Malawi, fuel stations, and airports (Interview 2011).

\section{Assistance to Quit Tobacco Use}

This measure was analyzed using a five scale indicator that focused on the presence of nicotine replacement therapy or smoking cessation program that were either sponsored or not. The evidence from available data places Ghana within category four and Malawi at the category two. Ghana is a member of the international "quit and win cessation program" that offers incentive to smokers to quit. The cost and incentives to encourage the quitting of smoking are covered by the Ministry of Health and the Ghana Health Service. Malawi was placed in the second category because no such program or any other was reported in the country (Interview 2011). 


\section{Product regulation and ingredient disclosure}

Articles (9), (10) and (11) of the FCTC requires parties to adopt a law or some measures for testing and measuring as well as disclosing the contents of tobacco products, and also to comply with the required health warning for tobacco products to be imported into the country. Ghana ranked within the fifth category on a six scale indicator that focuses on the presence of health warning on cigarette packs, the position and size that the warning occupies, and whether specific health warning are mandated. Ghana has a voluntary agreement that requires the importers of the cigarettes to register and disclose the contents of cigarettes to be imported into the country. The Ghana Food and Drugs Board (FDA), per the 2007 directives of the Ministry of Health, ${ }^{40}$ mandates tobacco importers to register all tobacco products (MOH, GHS \& FCTC 2010). By way of monitoring, the importers are given a form to sign, on which they indicate the contents of the products to be imported into the country and are also required to test the ignition propensity of cigarettes.

The instructions on the forms indicate the required ingredients allowed for cigarettes imported into the country and the health warning to be indicated on the cigarette pack. Generally, the importers are obliged to carry one of three rotational sets of textual health warnings ${ }^{41}$ on both the front and back of each cigarette pack (VALD 2010; MOH, GHS \& FCTC 2010; Interview 2011; also refer to Figure 6.1). The Ghana Food and Drug Board has since 2010 been enforcing a requirement insuring that the size of the message covers $50 \%$ of both the front and back of the pack and (FCTC 2010). The warning should also be clear, indicated in black and white, visible and legible, and written in English, the official language of the country (MOH, GHS \& FCTC

\footnotetext{
40 The Ministry of Health directed that all importers should comply with the requirement of the FDA about the regulations and required content of tobacco products allowed in the country.

${ }^{41}$ The health warning was developed by the National Tobacco Control Steering Committee and regulated by the FDA
} 
2010; Interview 2011). Moreover, all retailers are required to display a common warning of the Ministry of Health at all point of sales (Interview 2011, also refer Figure 6.1). In addition, the Minister of Health has indicated that the FDB is also considering appropriate pictorial health warnings for tobacco packs to display (Interview 2011; FCTC 2010). The Northern Command of Custom, Excise and Preventive Service (CEPS), has on certain occasions seized and destroyed certain cigarettes imported into Ghana because it did not have the mandated Ministry of Health warning clearly indicating the harmful effects of smoking (Interview 2010).

Malawi, on the other hand falls within the second category of the scale because of the absence of the nationally required health warning. An interviewee indicated that "Malawi does not legally require health warning on cigarette packs, but the companies put a one line sentence after all the products notification that smoking maybe hazardous to your health". He observed that the health warning is written in very small letters that is not easily noticeable (Interview 2011). Given that Malawi does not legally require health warning on cigarette packs, the companies use the health warnings to boost their corporate image - an indication of how they care about the health of the public - but not for the public health concern.

\section{Restrictions on advertising, promotion, and sponsorship}

Both countries have voluntary tobacco control agreements that prohibit the advertisement of the tobacco products and tobacco sponsorship for sports and recreational activities. Per article (13) of the FCTC, all parties are required to adopt a comprehensive ban of all advertising, promotion and sponsorship. Ghana ranked four on a six scale category that analyzed the instrument based on the media and other indirect forms of advertising where the ban is required. 
Figure 6.1 Sample Health Warnings on Cigarettes and Point of Sale in Ghana

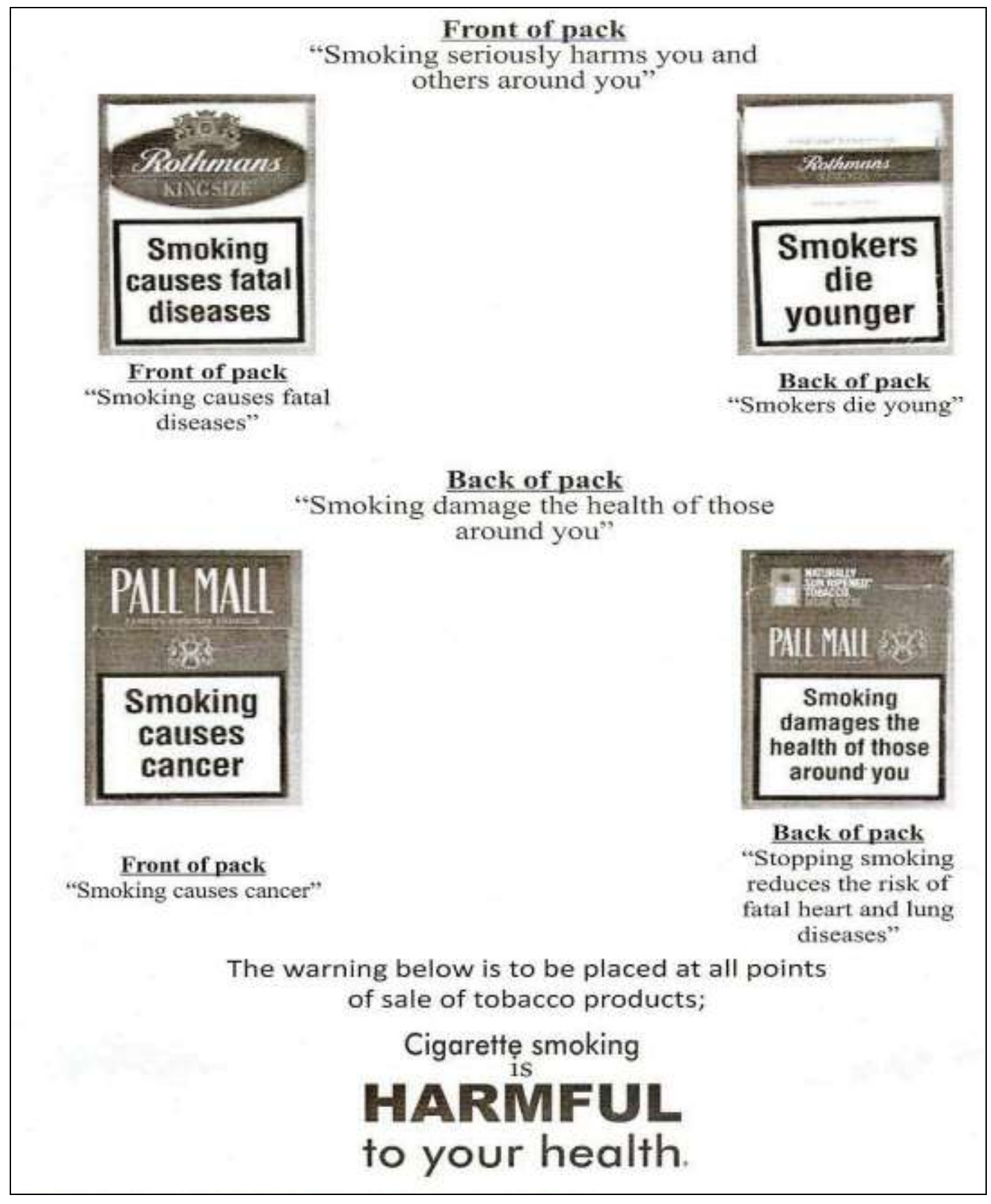

Source: (VALD 2010; Opuni 2009) 
Since 1982, Ghana has successfully banned all cigarette advertisements of any form on radio, television, and the print media (Interview 2011; MOH, GHS \& FCTC 2010; Wellington et al 2011). The requirement of the National Media Commission for broadcasting indicates that smoking should only be shown when it is meant for plot and character development (MOH, GHS \& FCTC 2010).

Among the Ghana Food and Drug Board's requirements for the importation of tobacco products into the country is a ban on tobacco advertising on billboard, vehicles, and transportation stations or on the walls. The requirements also prohibit tobacco sponsorship for events and the offer of promotional samples of cigarettes to underage children. Nevertheless, point of sale advertising, outdoor and bill board advertising as well as sponsorship of certain events are allowed under stringent restrictions (MOH, GHS \& FCTC 2010).

On the other hand, Malawi fell within the category three because it has a voluntary agreement on a total ban of advertisement of tobacco products in only radio and television but allows advertisements carried in the print media to be accompanied by a health warning (Makoka et al 2011). An additional indicator was created to take care of the Malawian case, which was not covered by the original scale of the WHO. The original WHO indicator had a five scale, which had category three as ban on TV, radio and print media but Malawi has ban on TV and radio but not in the print media. Therefore, the indicators were increase to a six scale to make room for the Malawian case.

\section{Price and Tax Instruments}

Price and tax measures are considered as one of the effective instrument for controlling tobacco consumption. The conventional wisdom of tobacco control is that the increase of excise 
tax on tobacco will increase the price of the product and make it inaccessible to marginal smokers with limited income. Article six of the FCTC treaty recognizes price and tax measures as effective means for reducing tobacco consumption by all cohorts of society, especially the youth. Both Ghana and Malawi ranked within the category four of the five scale indicator for the tax measure. The countries have both increased excise taxes on tobacco products, but the intention for the tax increase was to raise revenue (Interviews 2010/2011). Ghana has 67.5\% total tax as a proportion of cigarette price and that of Malawi is 53.89\%. However, the excise tax as a percentage of cigarette prices are $14 \%$ and $37.3 \%$ for Ghana and Malawi respectively (Eriksen, Mackay and Ross 2012).

The government of Ghana imposed an import duty of a flat ad valorem rate of $140 \%$ of the Cost, Insurance, and Freight (CIF) value on cigarettes but the rate was changed in 2007 and replaced with a varied excise tax system for different brand of cigarettes (MOH, GHS \& FCTC 2010). However, the pre -2007 ad valorem flat rates of $140 \%$ of the CIF value was re-adopted in 2009 (MOH, GHS \& FCTC 2010). Wellington et al (2011) maintain that the excise tax system was abolished after the relocation of the last tobacco manufacturing plant to Nigeria. Moreover, the Ministry of Finance has increased the import duty to $150 \%$ recently (Interview 2011).

In addition, there is a value added tax (VAT) of $12.5 \%$ and a health insurance levy (NHIL) of 2.5\% which are imposed on all goods (Wellington et al 2011). Tobacco products also attract 0.5\% levies of the Economic Community of West African States (ECOWAS) domestic export development and investment fund (EDIF) (Wellington et al 2011; MOH, GHS \& FCTC 2010). The seemingly high taxes on tobacco product are not supposed to serve as punitive measure to control tobacco consumption as the taxes are mainly for revenue (Wellington et al 2011). However, some tobacco control activists maintain that price of cigarettes are still cheap in the 
country compare to countries like Canada and the UK, and call on the government to pass the draft tobacco bill into laws to institute high taxes that will deter smokers from smoking (VALD 2010).

On the other hand, the objective of the tax increase in Malawi was to promote the purchase of Malawian tobacco leaf. Therefore, different excise tax rates have been instituted for cigarettes that use Malawian tobacco leaf and those that do not. The rates on the tobacco using the Malawian tobacco leaf are lower. The tobacco control activists believe that the small tax increase in both countries may cause some smokers to quit and is an achievement that can be built upon. It cannot be denied that the rates could still serve a dual purpose of compelling marginal smokers to quit due the high excise and other taxes imposed on the tobacco products while at the same time raising revenue in both Ghana and Malawi.

In addition to the demand reduction measures discussed above, other instruments are also analyzed below:

The provision of support for economically viable alternatives

Article (17) of the FCTC protocol mandates parties to assist tobacco farmers and workers with economically viable alternative crops to farm or jobs to do. In both Ghana and Malawi, minimal efforts to provide alternative economic activities for tobacco farmers have been adopted. In Malawi, with the help of a grant from the US government under the USADF, tobacco farmers have started to grow other products such as tubers. In Ghana, where the tobacco farming has been shrinking after the closure of the BAT manufacturing plant, the Ministry of Food and Agriculture (MOFA) with assistance of the World Bank, offers limited loans to farmers to undertake alternative farming activities (Interview 2011). However, the tobacco control activists argue that the credit offered to the farmers is not enough to motivate the farmers to completely 
stop the growing of tobacco leaves because the farmers do not get enough credit to acquire big farmland for enough crops that they can sell (Interview 2011). Normally, the farmers end up feeding on the crops they grow and do not get to sell some of the crops for income. However, with tobacco farming, they are able to obtain enough income to buy food and sometimes save.

\section{Education, communication, and public awareness on the consequences of smoking}

Perhaps one measure that is vigorously undertaken is public education on the harm of tobacco to public health. Article (12) of the FCTC requires parties to adopt programs that will promote effective and comprehensive educational and public awareness on the harm associated with tobacco consumption and second hand smoking as well as to offer assistance for quitting smoking. Both Ghana and Malawi undertake active public education on the negative consequences of smoking. The Ministry of Health of each country uses the Annual No Tobacco Day, celebrated on May 31, to educate the public about the harmful effects of tobacco consumption. The Ghana Health Service also uses the Quit and Win campaigns to make people aware of the benefit of tobacco cessation and a tobacco free lifestyle. Other Ministries and governmental agencies also offer educative programs on the tobacco control.

For instance, the Ghana Ministry of Education, with the assistance of the Ghana Health Service, offers educational programs on tobacco control in schools (Interview 2011). Pictorial information known as the smoker's body posters are distributed to schools for the purposes of making the students and teachers aware of the dangers associated with smoking (MOH, GHS \& FCTC 2010). In addition, local NGOs, some media groups, and tobacco control organizations periodically organize public education on the effects of smoking on public health. For instance, NGOs like VALD of Ghana and YASED, which operates the Smoke Free Malawi project, 
periodically organize programs to educate the public on the harm of smoking. The Smoke Free Malawi project has media practitioners and health professionals as members, and they collaboratively undertake campaigns to educate the public on the harm associated with tobacco use.

\section{The establishment of a national coordinating mechanism for tobacco control}

The establishment of a national coordinating mechanism for controlling tobacco is considered essential to an effective tobacco control program. Article (5) of the FCTC recommends the establishment and financing of a national coordinating mechanism or a focal point for tobacco control. For both countries, the Ministry of Health spearheads tobacco control activities. In 2002, Ghana established the National Tobacco Control Steering Committee (NTCSC), which is tasked with adopting the appropriate mechanism for controlling tobacco in the country. The committee is also responsible for drafting a tobacco control bill for passage into law by the Parliament of Ghana and had since 2005 produced a draft bill, which has been incorporated into the Public Health bill currently in Parliament. Moreover, a tobacco control focal point is situated in the Research and Development Division (RDD) of the Ghana Health Service (MOH, GHS \& FCTC 2010).

On the other hand, in Malawi, in addition to the Ministry of Health that champions tobacco control activities, an NGO known as the Youth Alliance in Social and Economic Development (YASED) is serving as an umbrella body that hosts the Smoke free Malawi project. The Smoke Free Malawi program has a number of tobacco control governmental and nongovernmental organizations and activists as members and they have been putting pressure on the government of Malawi to sign and ratify the FCTC protocol, and also to adopt tobacco control legislation that 
is effectively enforced (Interviews 2011; Makoka et al 2011). However, YASED is only a NGO championing tobacco control activities but not an officially designated organization by government for coordinating tobacco control activities and mechanism.

Although Ghana and Malawi has not adopted any tobacco control legislation that is effectively enforced, many of the people interviewed believe that the interaction and collaboration with, as well as the funding from, the international nongovernmental and governmental organizations and agencies have played a major role in the minimal efforts in tobacco control achieved in both countries. The collaboration with the Ministry of Health and local NGOs has facilitated the adoption of the certain voluntary agreements on tobacco control, which could be built upon for effective tobacco control legislation. In the case of Ghana, some officials of the knowledge transfer agents were key-members of the committee that prepared the tobacco control bill (Interview 2010/ 2011).

\section{Litigation}

Litigation is also considered an instrument that could have impact on the activities of the tobacco industry and has emerged as a recent tobacco control tool in the developing countries. Given the fact that the judiciary is a component of the legal system and also litigation in some states in developed countries such as US was an effective tobacco control instrument, tobacco control activists see it as a possible instrument that can compel the government and tobacco companies to heed to their voice and adopt effective tobacco control law in the developing countries (Cairney et al 2012; Interview 2011). An interviewee in Malawi identified litigation as an instrument that could compel the government of Malawi to adopt tobacco control law. He maintained that a group known as Forum for a Clean Environment (FORCE) has sued the 
Ministry of Natural Resources Energy and Environmental Affairs, for failure to implement the environmental management act that mandates the regulation of tobacco smoke.

The interviewee also indicated that "litigation has so far greater chances of pushing for tobacco control agenda in the country than any other channel, as any court ruling will force the government to implement measures as contained in the court order without a chance. Litigation process will bring attention to the issues and evidence presented; courting much needed publicity to the tobacco control cause. Litigation in face of uncertainty by government is the only strongest available option for tobacco control in Malawi" (Interview 2011). However, the respondents in Ghana expressed doubt about the impact of litigation to promote tobacco control because of the influence of the government over the judiciary of the country. The impact of litigation as a tobacco control instrument in Ghana or Malawi is an interest that can be examine in detail in another academic study since that entails a detail examination of the legal systems in both countries.

\section{Impact of Voluntary Agreements as Tobacco Control Instrument in Ghana and Malawi}

Mostly, study conducted in western countries find voluntary agreement as tobacco control instrument to be ineffective (Studlar 2004, Cairney 2007). However, preliminary results from the two countries show that the voluntary agreement has achieved some progress as tobacco control instruments, especially in Ghana. For instance, the last tobacco manufacturing plant in Ghana left the country in 2006 because of the tobacco control activities, which satisfies the objective of the supply side of the tobacco control measures - to control the activities of the tobacco industry (Interview 2010, Owusu-Dabo et al 2010). The requirement for importers to disclose the content of cigarette products to be imported into Ghana is also well respected, which 
helps to regulate the activities of the importers (Interview 2010). On the demand side, a status report indicated that $43.3 \%$ of the participants who took part in the quit and win program in Ghana stayed tobacco free three years after the adoption of the program in 2002 (Wellington 2007).

In addition, report of recent surveys in both Ghana and Malawi show that majority of the people now consider the tobacco issue as public health issue instead of the political economy image presented by the tobacco industry in the past (Makoka et al 2011, Wellington et al 2009). While a comprehensive analysis of the impact of these voluntary agreements was not the focus of this study, these indicators provide some evidence of the impact of the voluntary agreement in the countries, especially on the supply side control measures. However, an extensive study will help to determine the real impact of the voluntary agreements on the demand side control measures. Perhaps, the success of the voluntary agreement as tobacco control instrument could be attributed to the structure and influence of the bureaucracies of the countries. The fact the bureaucratic agencies are more comfortable with the implementation of administrative rules and they also serve as agent of continuity with much influence on the administration of the countries because of the past histories might be the reason why the voluntary agreements have impact in these countries than the west.

\section{Knowledge Transfer and Adoption of Domestic Tobacco Control Policies}

The adoption of the voluntary agreements in Ghana and Malawi have mostly been the result of the interaction of the Ministry of Health with transnational tobacco control actors at venues such as the meetings of the WHO, the Commonwealth, FCA, and also through the online resource, GlobaLink. Normally, the information on the best practices for controlling tobacco 
consumption are presented to the countries at such meeting and sometimes, the countries voluntarily adopt the ones that are relevant to their tobacco control situation (Mamudu 2005). In other instances, the knowledge transfer agents offer sponsorship and funding for the adoption of a specific instrument of tobacco control. For instance, the Bloomberg Foundation and Initiative offered funding to the VALD, an NGO in Ghana, to lobby for the adoption of pictorial warning as a tobacco control instrument in Ghana (Interview 2010). The director of VALD was influential in pushing for the passage of the tobacco bill currently before the Health Select Committee of the Parliament of Ghana. The bill has a significant section on pictorial warning. Therefore, it is reasonable to state that such forms of knowledge transfer involves some sort of indirect or negotiated coercion whereby the knowledge transfer agent requires the receiving country to adopt a certain form of tobacco control policy instrument of their preference.

In Malawi, the government received funding from the US under the United States African Development Foundation (USADF) in 2009 for promoting development, diversifying the economy, and increasing participation of groups in trade and investment activities. A core aspect of this initiative was specifically for promoting the growing of alternative crops to the tobacco leaf, based on the US expertise on maintaining diverse economic activities to support her economy. Even though the sponsorship did not directly indicate that it was for tobacco control, it is no doubt that promoting an alternative to the growing of tobacco leaf is a major measure recommended by the FCTC for controlling tobacco.

Many of the voluntary tobacco control agreements adopted through the directives of the Ministry of Health and the increases in the excise taxes on cigarettes in both countries were influenced by the international nongovernmental and governmental organizations and agencies such as the IDRC/RITC, the WHO, America Cancer Society, the International Union against 
Cancer (UICC), and the Framework Convention Alliance. These organizations champion the adoption of best practices and new tobacco control ideas such as high excise taxes, warning labels, and a ban on tobacco advertising and on smoking in public places. Moreover, the countries and organizations promote the adoption of specific instruments they consider relevant for controlling tobacco consumption and will achieve the same results as the western countries where they were initially applied (Asare 2007). So far, the knowledge transfer agents have not attained a lot of success with the adoption of the transferred knowledge into legislations that are well enforced. However, some of the instruments recommended have been adopted as voluntary agreements, especially in Ghana (Interview 2011). For instance, the Ministry of Health issued a directive for smoke free areas to be designated at certain public places following the call of the COP of the FCTC for the adoption of smoke free laws since 2007.

Other means through which knowledge have been transferred include direct involvement of the knowledge transfer actor in drafting tobacco control policies of the receiving countries and using the policy documents of the developed countries as a guide to draft the policy document of the receiving country. For example, as earlier indicated, Ghana used the tobacco control policies of countries like Canada, Finland, and South Africa as a guide in writing the draft tobacco control bill currently before Parliament (Wellington 2007, Interview 2011). An interviewee indicated that the members of the Ghana national tobacco control steering committee studied the tobacco control policies of these countries and modeled the Ghanaian bill along certain relevant aspects of the policies. Also tobacco control experts from the WHO and the developmental partners of Ghana were brought in to offer advice to the committee that wrote the draft tobacco bill (Wellington 2007). It needs to be stressed that the voluntary agreement adopted so far are as 
a result of the engagement of the knowledge transfer agents with governmental institutions such as the Ministry of Health to influence them to adopt the tobacco control instruments.

\section{Liberal - Constructivism and Adoption of Domestic Tobacco Control Policies}

The battle between tobacco industry network and tobacco control activists over tobacco control is not just about the adoption or non-adoption of tobacco control laws but more importantly about shaping the understanding of the tobacco issue. The tobacco industry network wants the issue to be understood and perceived as that of political economy and has been projecting the economic importance of tobacco products to the economic development of these countries (Mamudu et al 2008; WHO 2008). On the other hand, tobacco control activists refute the political economy argument and highlight the negative health hazards of cigarette smoking to both smokers and non-smokers. The activists organize campaigns and educational programs to stress the health hazards associated with tobacco consumption. Normally, tobacco control activists in both countries use the Annual No Tobacco Day to highlight the dangers of smoking and educate the public on how to avoid the harm associated with smoking and also put pressure on policy makers to adopt relevant policies that will protect public health. The celebration becomes a form of symbolic politics, whereby the harm caused by tobacco consumption somewhere is highlighted to seek support for the adoption of tobacco control policy in the domestic arena (Keck and Sikkink, 1998).

In the past the tobacco industry network was successful in convincing a majority of countries about the economic importance of tobacco (World Bank 1999). However in recent times following the publication of the World Bank ground breaking study rejecting the claim of the tobacco industry about the economic importance of tobacco for the development of the 
developing countries and the findings of other studies providing more data to support the harm associated with tobacco consumption, there is evidence that this perception and understanding is shifting in support of the adoption of tobacco control laws to protect public health (Mamudu et al 2008; WHO 2008). Evidence from both Ghana and Malawi found support among majority of the public for the adoption of tobacco control laws that are effectively enforced to protect public health.

In Ghana, a recent survey conducted in one of the main tobacco growing areas indicated the willingness of farmers to grow alternative crops because of the negative health hazards associated with tobacco leaf growing $^{42}$ (IDRC 2009). Another survey found a large support among the public for the adoption of stricter tobacco control policies that are well enforced particularly to prevent the sales of cigarettes to minors and enforce a total ban of smoking in public places (IDRC 2009). One of the surveys, which were conducted by Ms. Edith Wellington of the Ghana Health Service and three other colleagues, had a total population of 308 participants, and some of the participants included members of Parliament and other policy makers. They used survey questionnaires on 242 participants and in-depth interviews on 66 participants to collect data. The survey found majority of Ghanaians, including some policymakers, to be concerned with the problem of tobacco consumption and its health hazards, especially youth smoking. Also, the Chairman of the Parliamentary Select Committee on Health and the Minister of Local Government and Regional Development in 2009 have called for the hastening of the passage of the tobacco control bill into law to protect public health, especially the hazards of second hand smoking (Odoi-Larbi 2009). The story is not different in Malawi, where a similar survey found support of the public for the adoption of tobacco control laws that

\footnotetext{
${ }^{42}$ Details earlier discussed under Liberal-Constructivism and Tobacco Control in Ghana at page 174.
} 
are effectively enforced to protect public health. The survey conducted by researchers found that $80 \%$ of non-smokers and over $50 \%$ of smokers in Malawi would support a total tobacco advertising ban in Malawi (Makoka et al 2011).

Tobacco experts and tobacco control activists believe that the change in behavior and perception of certain policymakers as well as the recent public support for the adoption of tobacco control policies that are properly enforced is the result of the tobacco control information and educational program, which highlights the harm of tobacco smoking to public health. Some of the people interviewed indicated that the public now understands the tobacco issue as a public health issue due to the message that they have been receiving from tobacco control actors and programs. The campaign of the tobacco control actors is generating support for the adoption of tobacco control law. It is generally believed that a change in the understanding of the tobacco issue is a necessary precursor to the adoption of tobacco control legislation that can be effectively enforced (Interviews 2010/2011)

\section{Findings}

So far the theories adopted for this study seem to provide a detailed insight and a better understanding of the politics surrounding the adoption of tobacco control policies in Malawi and Ghana. Some studies have demonstrated how international governmental and nongovernmental actors collaborate with actors within a country to ensure the adoption of a certain policy (Asare 2009, Keck and Sikkink 1998, Farquharson 2003, Hudson 2001, Trubek et al 2000, Mamudu and Studlar 2009). For instance, Keck and Sikkink (1998) found that international NGOs and governmental actors collaborated to push for the adoption of human rights and women's right laws in certain countries. 
Malan and Leaver (2003) maintain that the success of South Africa's tobacco control following the 1999 Tobacco Control Amendment Act was partly the result of anti-smoking groups and nongovernmental organizations putting pressure on policy makers to adopt domestic laws. Asare (2009:103) identifies some of the anti-smoking groups in South Africa as the National Council against Smoking (NCAS), the South African Medical Research Council (SAMRC), the Cancer Association of South Africa (CANSA), the Heart and Stroke Foundation of South Africa (HSFSA). Asare (2009) argues that the anti-tobacco groups in South Africa collaborated with intergovernmental and nongovernmental organizations such as the WHO, World Heart Federation, International Union against Cancer, and the World Conference on Tobacco or Health to ensure the success of its campaign.

Asare (2009), and Malan and Leaver (2003) maintain that the groups lobbied persistently and waged a relentless war to protect both smokers and nonsmokers from the harm of tobacco before the government became involved in the tobacco control in the 1990s. They further argue that the lobbying, combined with the subsequent commitment to public health by the African National Congress government led by Nelson Mandela, resulted in the success of the tobacco control in South Africa. Moreover, Waverly (2007) maintains that the organizations helped South Africa to adopt tobacco control instruments, including price and taxation tobacco control measures as a strategy to control the consumption of tobacco products. This was in spite of the strong opposition from the tobacco companies.

This study illustrates how governmental and nongovernmental organizations are actively collaborating with domestic actors in Ghana and Malawi to push for the adoption of their interest as tobacco control laws. Additionally, this study finds that, in spite of the failure of these countries to officially adopt tobacco control legislation that are properly enforced, certain 
voluntary agreements have been adopted through the directives of the Ministry of Health (and other government ministries in Ghana) in the face of opposition by the tobacco industry network. Moreover, the tobacco bill in Parliament has tobacco provisions that are based on the best practices and new ideas of tobacco control of some countries with effective tobacco control laws (Wellington 2007).

Similarly, while some studies have found that policy adoption can occur through voluntary policy transfer, policy copying, policy borrowing, or lesson drawing (Rose 1991, 2005, Studlar 1999; Newmark 2002; Bennett 1991), others have shown that policy adoption can be as a result of negotiated policy transfer processes (Asare 2005; Evans 2004). In addition, other studies found that the adoption of certain policy issues can occur through a combination of both voluntary and coercive policy transfer strategies (Evans 2004; Evans and Davies 1999; Ladi 2005; Common 2001; Dolowitz and Marsh 1996; 2000).

The findings of this study show that the tobacco control knowledge transfer in Ghana and Malawi and probably all developing countries, is facilitated by both voluntary and indirect coercive policy transfer processes, which to a large extent confirms the findings of previous studies. It is obvious that the knowledge transfer agents such as the ACS, the Bill and Melinda Gates Foundation, the Bloomberg Initiative and Foundation, IDRC/RITC, WHO, Commonwealth, and IUTLD, etc., are using both direct and indirect monetary incentives to engage the bureaucratic institutions - Ministry of Health- to promote the adoption of preferred best practices of tobacco control as domestic policies in Ghana and Malawi. Simultaneously, the countries also voluntarily adopt certain policies relevant to their policy problem, which are considered safe for the political capital. For instance, in Malawi, the adoption of the child labor laws that prohibit children from working on the tobacco farms was safe for the politicians 
because it was not a direct tobacco control policy; even though it addresses the issue of child exposure to high levels of nicotine.

Lastly, earlier studies using the liberal - constructivist approach to international relations have demonstrated how relevant information / new ideas can be disseminated to certain areas to change the understanding of certain policy issues (Wendt 1992, Haas 1990, Wolfson 2001, Nathanson 1999, Finnemore 1996, McNamara 1998, Goldstein and Keohane 1993). Mamudu (2005) argues that the measures for controlling the serious health hazards attributed to tobacco consumption is socially constructed by tobacco control advocates. However, the measures have been projected into the international arena as effective measures for controlling the harm associated with tobacco consumption. The adoption of voluntary agreements as tobacco control instruments can be credited to how the transnational tobacco control actors are promoting the adoption of those measures on the global scene to convince the policymakers, especially in the developing countries to believe that the failure to adopt those measures will create a public health epidemic.

The findings on how knowledge / new ideas / information on the health hazards of tobacco consumption is being used to shape a new understanding of the tobacco issue in Ghana and Malawi in a way that it motivate the adoption of tobacco control instrument confirms earlier studies done under the liberal-constructivist approach. The transnational tobacco control actors are vigorously engaging the two countries to adopt tobacco control law that will prevent future tobacco epidemic. In addition, the domestic tobacco control actors in each of the countries also actively use the media and other forms of campaign to educate the public about the health hazards of tobacco consumption. This study finds that the actions of the tobacco control actors is generating increasing level of public support for the adoption of properly enforced tobacco 
control legislation in both countries because of the perceived fear associated with the failure to adopt the tobacco control instrument that the transnational agents have made them to believe are effective for curtailing a tobacco epidemic (Makoka et al 2011; Odoi-Larbi 2009; IDRC 2009).

\section{Structure of Bureaucracy and Adoption of Voluntary Agreements}

The success of the voluntary agreements as tobacco control instruments is also attributable to the structure and influence of the bureaucratic agencies, especially the Ministry of Health of the countries which have been spearheading the adoption of tobacco control measures. The bureaucrats are the main implementers of the policies of the government and mostly they are interested in the adoption of administrative rules that they have inputs in and are easier to implement. Therefore, the adoption of some of the voluntary agreements is preferred by the bureaucrats to address the tobacco problem because the interest groups that maybe against the adoption of the instrument pose less resistance during implementation due to their initial involvement in the process. The involvement of the tobacco interest groups in the negotiations for the adoption of policy instrument helps to ensure some level of compliance during the implementation of the voluntary agreements, especially in the case of Ghana.

\section{Model Testing and Discussion}

The preceding analysis indicates that the combination of all the three theories help to understand the processes leading to the adoption of domestic tobacco control laws in Ghana and Malawi and perhaps other developing countries in sub-Saharan Africa. It is clear that the knowledge transfer agents use both direct and indirect incentives to engage the Ministry of Health that are spearheading the adoption of the voluntary agreements. In spite of the use 
incentives of one form or the other to motivate the adoption of the preferred tobacco control laws promoted by the tobacco control agents, the receiving countries also willingly adopt certain policies that they consider as relevant for their policy environment. In addition, the collaboration between international and domestic knowledge transfer facilitates the adoption of the interest of the tobacco control agents - which are being promoted as effective tobacco control ideas and best practices for controlling the anticipated harm associated with the tobacco products - into laws in the countries. Finally, the dissemination of tobacco control information is leading to a change in the way the tobacco issue is understood, which is facilitating a change in the behavior of the countries to adopt some voluntary tobacco control measures or adopt some laws that indirectly promote tobacco control. The changes in behavior also take the form of a change in the rhetoric of policymakers about the tobacco control.

However, not all the propositions for this study were validated. The propositions under the multi-level knowledge transfer approach included the notion that: 1) incentive indirectly promote the adoption of tobacco law; 2) strong external and domestic pressure facilitate the adoption of tobacco control law; 3) Commonwealth meetings and interactions generate the adoption of tobacco law 4) the lobbying activities of BAT among member countries of the Commonwealth prohibits the adoption tobacco law; 5) signing and ratifying FCTC protocol promotes the adoption of tobacco law; 6) participation in formal or informal tobacco control conferences and meetings promote the adoption of tobacco law; and, 7) the activities of transnational tobacco control IGOs and NGOs influence the adoption of tobacco law.

The propositions were examined with the data collected from the responses to the interviews and secondary document provided by some of the officials who were contacted and archival sources. The analysis of data provides evidence in support of the proposition that 
indirect coercive policy transfer is a major component of the tobacco control knowledge transfer taking place in the developing countries. The tobacco control knowledge transfer agents use financial and other form of incentives to influence the Ministry of Health of Ghana and Malawi to adopt tobacco control law as means of conforming to the norms of the tobacco prohibition regime, FCTC. Secondly, it was found that active role of both international and domestic tobacco control actors, especially the bureaucratic agencies are essential to the adoption of tobacco control instruments (voluntary agreements) in both Malawi and Ghana. However, the domestic actors are influential and make impact when policymakers are not directly involved in the tobacco business. For instance, in Malawi, where key policy makers own tobacco farms, the few voluntary agreements to control tobacco is being ignored by a tobacco manufacturing company recently established in the country. On the other, there are constant attempts to strengthen the voluntary agreements in Ghana because of the absence of policymakers who are directly involved in the tobacco business.

Simultaneously, the countries are introduced to tobacco control ideas through their participation in the conferences of IGOs such as the WHO and the Commonwealth, and the online tobacco control community, GlobaLink. Therefore, the countries have voluntarily adopted some of the ideas as tobacco instruments in the form of voluntary agreements and other laws that they consider relevant for their tobacco control situation. Moreover, the transnational tobacco companies through their activities and interactions with the countries also influence the adoption of the tobacco instruments. Thirdly, the study did not find any evidence to support the notion that the presence of the headquarters of the British American Tobacco Company in Great Britain and possible lobbying of Commonwealth member countries has any impact on the decision of the member countries not to adopt tobacco control law. Instead, it was found that the tobacco 
industry uses their affiliates in any country to obstruct the attempt to adopt any policy that will decrease their profit making and they are mostly successful when the country has a tobacco economy.

The study also found evidence in support of the notion that signing and ratifying the FCTC influences the adoption and implementation of legally backed tobacco laws. The signing and ratifying the FCTC protocol increases the pressure on the government, which forces the country to make some progress with adopting some tobacco control instruments. For instance, the Parliament of Ghana is currently debating a draft tobacco bill that is likely to pass into laws before the end of the term of the NDC government in December 2012 (Interview 2011). Moreover, Ghana has adopted and continuously strengthened voluntary agreements to control the consumption of tobacco and to prevent its health hazards. In addition, the last tobacco manufacturing plant was forced out of the country because of unfavorable conditions for operation. Tobacco control activists maintain that Ghana's action is because of its obligation to the FCTC treaty (Interview 2011).

On the other hand, in Malawi, where the country has not signed or ratified the treaty, the few tobacco control instruments adopted through the directives of the Ministry of Health is increasingly being rendered ineffective. The country has allowed a tobacco company to be established in 2009 and the company is actively disobeying the ban on advertisements (Interview 2011). The company is alleged to have mounted a huge billboard at the industrial area of the capital and is using deceptive tobacco warnings as well as giving away free samples of cigarettes to the youth (Interview 2011).

The propositions under the global advocacy network theory revolves around the idea that: 1) countries will adopt tobacco control policies if the knowledge is transfer by collaboration 
between IGOs and NGOs; 2) Tobacco growing economy are less likely to adopt effective tobacco law; 3) Countries with tobacco manufacturing plant are less likely to adopt effective tobacco laws; and 4) Tobacco economies are more likely to adopt laws that indirectly control the consumption of tobacco. The analysis of the data finds evidence in support of the proposition that the knowledge transfer agents comprising IGOs and NGOs mostly act in a group to transfer the knowledge. The composition of the actors takes different form including the collaboration between actors who actually visits the countries and those that donate money for tobacco control in those countries. The agents transferring the tobacco control knowledge to the developing countries normally do so in coalitions and pull resources together to transfer the knowledge to the countries. Therefore, it could be inferred that acting in coalition is an effective means for knowledge transfer. Nonetheless, there is no evidence suggesting that individual organizations will not be successful with the knowledge transfer to the developing countries.

It was also found that the tobacco growing and manufacturing economies are reluctant to adopt stricter tobacco control laws because of the belief that it will decrease the contributions to their GDP and also because of the reaction of the tobacco farmers and workers as well as policy makers who have investments in the tobacco industry to such a policy. However, the countries are likely to adopt laws that indirectly address the tobacco issue. For instance, the Parliament of Malawi has passed labor laws that indirectly address the issue of children exposure to high levels of nicotine from working on the tobacco farms, even though it is unwilling to pass any direct tobacco control legislation. In the case of Ghana, the Parliament has not enacted any tobacco control law but is currently debating such law five years after the last company moved out of the country. However, the Ministry of Health has issued several directives for certain tobacco 
control instruments such as advertising ban that are well respected in the country partly due to the fact that tobacco was not a major contributor to the GDP of the country.

Lastly, the propositions under the liberal-constructivist approach included the notion that: 1) the tobacco control information is changing the understanding of the tobacco issue; 2) the transnational actors are using tobacco control information to promote conformity to the norms of the tobacco prohibition regime. The study found evidence in support of the notion that the tobacco control campaign is changing the perception of the public about the tobacco issue in Malawi and Ghana as recent research show that majority of the public now support the adoption of some form of tobacco legislation to protect public health. In addition, the activities of some transnational tobacco control actors provide evidence to support the notion that they are promoting conformity with the FCTC protocol, which can be considered an established tobacco prohibition regime. The activities of the actors show an attempt to change the thinking and behavior of the countries towards cooperation for a mutually beneficial objective, which is a core characteristic of a prohibition regime (Nadelmann 1990; Studlar 2002). The activities to change the understanding on the tobacco issues also accounts for why the countries are becoming receptive to certain tobacco control ideas and are also adopting certain tobacco control instruments.

\section{Limitation of the Study}

A major limitation of study is the absence of tobacco legislation passed by the National Parliaments of the countries. The absence of tobacco control law legislated by the Parliament necessitated focusing on voluntary agreement instituted through directive of the Ministry of Health and other relevant ministries. The adoption of this strategy was influenced by the 
objectives of the FCTC treaty, which requires parties to adopt laws, regulations, administrative directives and other tobacco control measures to promote the treaty (FCTC 2009). Nevertheless, this study emulates the strategy of Drope (2010), in which he examined the adoption of smoke free measures in certain African countries using alternative tobacco control arrangements. Therefore, this study used the adoption of voluntary agreements, alternative policy instruments that promote the spirit of the FCTC, and the changes in the rhetoric of policymakers as an indication of the trend of the policy direction of the countries. The strategy adopted helped to provide the picture of the politics of tobacco control in the two countries. It is not certain if the replication of the study will produce the same findings. Nevertheless, the findings might be generalized if measured against the FCTC provisions.

In addition, the sampling of interviewees can also affect the generalizability of this study as the desire to interview as many people as possible, randomly selected from each of countries and the organizations, was not met. As a result, a purposive and snowball sampling method was used to obtain participants for the interviews, which enabled me to select knowledgeable people to provide the required information for the study. In the end, the strategy took away the randomness that makes the study generalizable. Moreover, attempts to interview some people from the tobacco industry network and the economic departments in the two countries as well as officials from the Commonwealth Secretariat were unsuccessful. Therefore, the information on the groups and organizations were obtained from reports, published materials and information from their websites. The final sample size was small and biased, which may also have negative impact on possible generalization and replication of the findings of the study. 


\section{Future Research}

This study contributes to the studies that examine the politics of tobacco control in developing country but specifically focus on Ghana and Malawi, two Commonwealth countries in sub-Saharan Africa with a centralized form of government and influential bureaucratic institutions. It also shows how voluntary agreements often considered an ineffective tobacco control instrument in western countries could be used to control tobacco in areas where the National Parliament fails to enact tobacco control. Therefore, future studies can examine if the findings will be applicable to other English countries, sub-Saharan African countries, nonCommonwealth, or Commonwealth countries with federal system of government where policymaking authority is shared with local levels of government but the agencies of the central government still wields ultimate authority. Given that tobacco control activists have multiple points to influence the adoption of policy in federal systems, future research could be design to examine the politics surrounding the adoption of domestic tobacco control policies in such countries.

Alternatively, other research could examine the politics surrounding the adoption of tobacco control laws in the Francophone countries to ascertain if the findings will be similar or different from what were found in these two countries. Finally, other research could investigate the impact of the voluntary agreements on tobacco smokers. Since these agreements are mostly between tobacco interest groups and the agencies issuing the directives, a study could be designed to find out if the directives generate any reduction in the number of smokers or not, in spite of the notion that they are weakly enforced. The findings of such a study will help to ascertained if adopting the voluntary agreements is necessary or a waste of resource. 


\section{Appendix}

\section{Interview Questions and Protocol}

\section{Background}

Following the success of tobacco control in most developed countries, the tobacco companies have shifted their attention and activities to the developing countries because many of these countries have weak tobacco control laws or do not have any. This has resulted in a rapid increase in tobacco consumption and its related health hazards. The adoption of the best practices of the tobacco control measures by developing countries have been identified as the best means of addressing the tobacco epidemic. There is some evidence that the officials of U.S, Canada, WHO, NGOs, and epistemic communities are contacting the developing countries to transfer the tobacco control knowledge to them so that they can adopt relevant tobacco control policies to avoid the epidemic. This study examines the policies being adopted in Ghana and Malawi and the political struggles that characterize the process.

(1) Please identify the capacity in which you are/were involved in the tobacco control activities, and the main activities of the group, organization, or country that you represented.

(2) Have you ever engaged in tobacco control related interactions with any individuals, groups, countries, or organizations? If so, please specify and explain the purpose of the interactions and indicate if the interaction was official or unofficial.

(3) Which countries, international governmental organizations (IGOs), nongovernmental organizations (NGOs) or experts were involved in the interactions?

(4) Please indicate the number of times you were engaged in the interactions and explain briefly the specific tobacco related issues that were discussed.

(5) Kindly indicate the format (meeting, conferences, etc) of the interactions and the organizations, countries, or groups who organized, initiated or led the discussions.

(6) If you were engaged in more than one interaction, were they with the same actor(s) or different group of actors?

(7) If the interactions were with different actors, were the same types of issues discussed? Please identify the issues discussed during each engagement.

(8) Were there other means - such as correspondence through email or reading of relevant literature - through which you received information on tobacco control issues? Please identify the means and the specific information you were introduced to. 
(9) How does the contribution of tobacco to the economy of your country affect the decision to adopt or not to adopt tobacco control laws?

(10) Do you think that it is necessary to encourage countries to become parties to the FCTC? If so why or why not?

(11) Do you think that there is a group promoting question (10) above. If so, kindly identify the group and your opinion on their activities?

(12) Do you consider the tobacco issue as global or local? Kindly indicate the extent to which you consider the tobacco issues as global or local?

(13) How do you perceive/understand the tobacco issue?

(14) Has this always been your perception/understanding of the tobacco issue? If not, what accounts for the change in your understanding of the tobacco issue?

(15) How has the interactions on the tobacco issue with other actors affected your understanding/framing of the issue?

(16) Does your country have domestic tobacco control laws? If so, what tobacco laws does the country have?

(17) What reasons influenced/motivated your country to adopt or not to adopt a particular tobacco control laws?

(18) Does the use of a common language influence your decision on the type of meetings or correspondence to be part to discuss issues about tobacco control? Please explain.

(19) Do you think the structure of bureaucracy can impact the adoption of tobacco control instrument in your country?

(20) Has your country signed and ratified the FCTC protocol?

(21) What measure do you consider to be the most important means or ideas to control tobacco?

(22) Have your country receive any incentive or funding to adopt any direct or indirect tobacco control instrument? If so, kindly explain the outcome.

(23) How do you consider the tobacco issue?

(24) Were the interactions on tobacco control that you were engaged in about specific or general ways to control tobacco? Explain.

(25) Is your country likely to adopt a tobacco control law if the idea is presented by a group of actors/organizations or individual actor/organization? 
(26) Do you think that the tobacco industry network can use IGOs to influence the adoption or non-adoption tobacco control policies? Please explain.

(27) Please identify the nature of tobacco control activities in your country.

(28) Kindly identify the composition of tobacco activists in your country. Please be sure to specify coalitions involving international, regional and domestic actors.

(29) Have your country adopted any law that can indirectly control tobacco production, consumption or exposure? If so, please explain why the country did that.

(30) Kindly discuss how domestic actors influence the adoption of domestic tobacco control law in your country?

\section{Types of Interviews Conducted}

\section{Telephone Interviews}

The interviews were conducted between March 2010 and July 2011. The respondents were contacted through the email to request their participation and also for them to indicate the possible dates and time for an interview. In addition, the emails made the respondents aware of the academic nature of the study and a copy of the letter approved by the IRB of West Virginia University was sent to them to assure them of their confidentiality. Once the person accepts to take part in the study, a copy of the research question was sent to him/her prior to the date of the interview to give the person an idea of the issues that will be covered and also help the respondent prepare. The interviews were recorded through hand written notes. Mostly, a request was made to the respondent for an email follow up to clarify certain issues that might have been skipped during the interviews. The interviews were informal and normally short once the respondents accept the email follow-up. This approach helped respondents to go into details on certain issues and also enabled them to talk about a whole range of issues of interest to them. 


\section{Face-to- Face Interviews}

These interviews were conducted at New York and New Jersey during the summer of 2010 from June 7-11. The interviews were mainly conducted with nongovernmental organizations who are involved with the tobacco control activities of the developing countries. Prior to the interview, responded were contacted through emails and telephone to schedule a date and time for the interviews. In addition, the emails made the respondents aware of the academic nature of the study and a copy of the letter approved by the IRB was sent to them to assure them of their confidentiality. The interviews were informal and they normally started with the establishment of rapport with the participants through general questions on the activities of the organization and the specific role of the interviewee. At the end of the interview, a request was made for follow-ups through emails.

\section{Interviews through Email}

Email correspondences were used than all the other means of collecting data for the study. Almost everyone who was interviewed through telephone and face-to-face also had follow-up interviews through the email. Many of them sent relevant information and documents as email attachments or referred me to relevant documents during the follow-up. The respondents were also able to make detail comments on a topic in the email and sometimes talk about other issues that were not mentioned in the initial contact. Some of the people in Malawi and Ghana, who declined to participate in the telephone interview, referred me to their publications or other documents on the issue. The email interviews also enabled me to ask specific questions to the respondent to probe for detail of a particular issue. 


\section{List of Individuals and Organizations that Provided Information/Document for the Study}

Kondwani Munthali, Media Consultant, Public Health and Youth Development Officer, and also National Coordinator, Youth Alliance in Social and Economic Development (YASED), Malawi

Mr. Issah Ali, National Coordinator of Coalition on the Tobacco Control Bill of Ghana, Executive Director of Vision for Alternatitive Development (VALD), Ghana and also, chairman of the African Tobacco Control Alliance (ATCA)

Dr. Marty Otanez ${ }^{43}$, Assistant Professor, Anthropology Department, University of Colorado, Denver

Dr. Jeffrey Drope, Assistant Professor, Political Science Department, Marquette University, Milwaukee, WI

Dr. Owusu-Dabo ${ }^{44}$, UK Centre for Tobacco Control Studies, Division of Epidemiology and Public Health, University of Nottingham, Clinical Science Building, City Hospital, UK

Ghana Health Service, Ministry of Health, Ghana

Department of Health, Malawi

Mr. Samuel Brenyah, Former Member of Parliament, Ghana

Dr. Armando Peruga, Program Manager, Tobacco Free Initiative, World Health Organization, Geneva, Switzerland

Dr. Barima Poku, Ghana Health Service, Ashanti Region, Ghana

Ms. Olga Bailey, World Health Organization, United Nation Office, New York American Cancer Society

Mrs. Betsy Fuller Matambanadzo, International Health Programs, Bloomberg Philanthropies and Initiative, New York

America Cancer Society

Ghana Food and Drug Board

Canadian International Development and Research Center/ Research for Tobacco Control Campaign for Tobacco Free Kids

\footnotetext{
${ }^{43}$ Dr. Otanez is a tobacco control researcher and expert on Malawi

${ }^{44}$ Dr. Owusu-Dabo has conducted research and written several articles on the state of tobacco control in Ghana
} 


\title{
Copy of Introductory Letter to Interview Respondents Approved by the Institutional Research Board of West Virginia University
}

\author{
TO WHOM IT MAY CONCERN
}

This letter is a request for you to take part in a research project to examine the processes of tobacco control knowledge transfer. This project is being conducted by Edward Brenya, a doctoral candidate at the Political Science Department under the supervision of Dr. Donley Studlar, an Eberly Family Distinguished Professor, Eberly College of Arts and Science, for a Ph.D degree in General Political Science. Your participation in this project is greatly appreciated and will take approximately 15 minutes.

Your involvement in this project will be kept as confidential as legally possible. All data will be reported in aggregate. The research will not ask any information that should lead back to your identity as a participant. Your participation is completely voluntary. You may skip any question that you do not wish to answer during the interview and you may discontinue at any time. West Virginia University's Institutional Review Board acknowledgement of this project is on file.

I hope that you will participate in this research project, as it could be beneficial in understanding the process of tobacco control knowledge transfer. Thank you very much for your time. Should you have any question about this interview or the research project, please feel free to contact the chair of the dissertation committee, Dr. Donley Studlar at (304) 293-3811, ext 5269 or by email through dstudlar@wvu.edu. The West Virginia University institutional review board can also be reached throughwvuecomp@wvu.edu or(304) 2937073.

Thank you for your time and help with this project.

Sincerely,

Edward Brenya 


\section{Explanation for Table 6.2: Status of Policies and Progress towards Selected Demand}

\section{Reduction Tobacco Control Policies/Measures}

\section{Monitor}

Definition: The implementation status of the monitoring measure was classified by grouping countries into four groups' indicators as follows:

$1=$ No known data or no recent* data or data that are not both recent* and representative**

$2=$ Recent $^{*}$ and representative** data for either adults or youth

$3=$ Recent* and representative** data for both adults and youth

$4=$ Recent $^{*}$, representative ${ }^{* *}$ and periodic ${ }^{* * *}$ data for both adults and youth

* Data from 2003 or later. $\quad * *$ Survey sample representative of the national population.

*** Survey repeated at least every five years.

Surveys were considered "adult surveys" if participants were above 15 years of age. Surveys were considered "youth surveys" if all participants were between 11 and 19 years of age.

Method of estimation: Country reported data, scored according to the definition of this indicator

\section{Protect from tobacco smoke}

Definition: Protect from tobacco smoke groups are calculated by scoring countries according to their responses to survey questions. The highest scores are allocated to countries achieving best practice. Scores for this indicator are calculated as follows:

$1=$ Data not reported/not categorized

$2=\mathrm{Up}$ to two public places completely smoke-free

$3=$ Three to five public places completely smoke-free

$4=$ Six to seven public places completely smoke-free

$6=$ All public places completely smoke-free (or at least $90 \%$ of the population covered by complete subnational smoke-free legislation)

\section{Offer help to quit tobacco use}

Definition: The implementation status of the Tobacco Dependence Treatment measure was classified by grouping countries into five groups. The groupings for this indicator are:

$1=$ Data not reported

$2=$ None

$3=\mathrm{NRT}^{*}$ and/or some cessation services** (neither cost-covered)

$4=\mathrm{NRT}^{*}$ and/or some cessation services $* *$ (at least one of which is cost-covered)

$5=$ National quit line, and both NRT* and some cessation services** cost-covered

* Nicotine replacement therapy.

** Smoking cessation support available in any of the following places: health clinics or other primary care facilities, hospitals, office of a health professional, the community.

\section{Warn about the dangers of tobacco}

Definition: The implementation status of the Health Warnings measure was classified by grouping countries into five groups. The groupings for this indicator are:

$1=$ Data not reported

$2=$ No warning or warning covering $<30 \%$ of pack surface 
$3=\geq 30 \% *$ but no pictures or pictograms and/or other appropriate characteristics**

$4=31 \%-49 \% *$ including pictures or pictograms and other appropriate characteristics**

$5=\geq 50 \% *$ including other appropriate characteristics $* *$ but no pictures or pictograms

$6=\geq 50 \% *$ including pictures or pictograms and appropriate characteristics**

* average of the front and back of the cigarette pack. ** ${ }^{*}$ Specific health warnings mandated;

- appearing on individual packages as well as on any outside packaging and labeling used in retail sale;

- describing specific harmful effects of tobacco use on health;

- are large, clear, visible and legible (e.g. specific colors and font style and sizes are mandated);

- rotate;

- written in (all) principal language(s) of the country

\section{Enforce ban on tobacco advertising}

Definition: The implementation status of the Tobacco advertising, promotion and sponsorship measure was classified by grouping countries into five groups. The groupings for this indicator are:

$1=$ Data not reported

$2=$ Complete absence of ban, or ban that does not cover national television (TV), radio and print media

$3=$ Ban on national $\mathrm{TV}$, radio but not print media

$4=$ Ban on national TV, radio and print media only

$5=$ Ban on national TV, radio and print media as well as on some but not all other forms of direct* and/or indirect** advertising

$6=$ Ban on all forms of direct* and indirect** advertising.

* Direct advertising bans:

- national television and radio;

- local magazines and newspapers;

- billboards and outdoor advertising;

- point of sale.

** Indirect advertising bans:

- free distribution of tobacco products in the mail or through other means;

- promotional discounts;

- non-tobacco products identified with tobacco brand names (brand extension);

- brand names of non-tobacco products used for tobacco products;

- appearance of tobacco products in television and/or films;

- sponsored events.

\section{Raises taxes on tobacco}

Definition: Taxes assessed include excise tax; value added tax ("VAT"), import duty (when the cigarettes were imported) and any other taxes levied. Only the price of the most popular brand of cigarettes is considered. In the case of countries where different levels of taxes applied to cigarettes are based on either length, quantity produced or type (e.g. filter vs. non-filter), only the rate that applied to the most popular brand is used in the calculation.

Given the lack of information on country and brand-specific profit margins of retailers and wholesalers, their profits were assumed to be zero (unless provided by the national data collector). The implementation status of the Raise tobacco taxes measure was classified by grouping countries into five groups. The groupings for this indicator are:

$1=$ Data not reported

$2=\leq 25 \%$ of retail price is tax

$3=26-50 \%$ of retail price is tax

$4=51-75 \%$ of retail price is tax

$5=>75 \%$ of retail price is tax. 


\section{References:}

“Accra Residents on New Tobacco Tax" Ghanaian Chronicle Friday, November 26, 2010.

African Tobacco Control Alliance 2009 “Annual Report 2009”

Agbenyikey, Wil "Ghana Parliament Discusses Tobacco Bill” Ghana News Agency, Sunday, 31 July 2011

Aguinaga, B. A., \& Shatenstein, S. 2002. Profits Over People: Big Tobacco Industry Activities to Market Cigarettes and Undermine Public Health in Latin America and the Caribbean. Washington, DC: PAHO.

Aidoo, Priscilla. 2009. “Tobacco Importation to be Banned" Ghana Ministry of Information http://www.tobacco.org/articles/country/ghana/?starting_at=15. Retrieved on 7/12/2010

Albaek, Erik. 2004. "Holy Smoke, No More: Tobacco Control in Denmark". In Ronald Bayer and Eric A. Feldman (eds.) Unfiltered: Conflicts over Tobacco Policy and Public Health. Cambridge: Harvard University Press. pp 190-218

Alder, Emmanuel. 1991. "Cognitive Evolution: A dynamic Approach for the Study of International Relations in their Progress". In Emmanuel Alder and Beverly Crawford (eds.) Progress in Postwar International Relations. New York: Columbia University Press

Alder, Emmanuel. 1992. "The Emergence of Cooperation: National Epistemic Communities and the International Evolution of the Idea of the Nuclear Arms Control". International Organization 46(1):101-145

Alder, Emmanuel and Peter M. Haas. 1992. "Conclusions: Epistemic Communities, World Order and the Creation of a Reflective Research Program" International Organizations, 46 (1): 367-390

American Cancer Society and World Lung Foundation. 2009. Tobacco Atlas Third Edition 
Tobacco Control.Org enews 2001 International Committee onSmoking Issues (ICOSI) http://tobaccodocuments.org.enews

American Cancer Society, Cancer Research, UK and International Union against Cancer "Global Advocacy for the Signing, Ratification, Implementation and Enforcement of the Framework Convention on Tobacco Control Executive Report

Anhari, Achadi, Widyastuti Soerojo, and Sarah Barber. 2005. "The Relevance and Prospects of Advancing Tobacco Control in Indonesia” Health Policy 72: 333-349

Arksey, Hilary and Peter Knight. 1999. Interviewing for Social Scientists: An Introductory

Resource with Examples. London; Thousand Oaks, Calif.: Sage Publications.

Aron, Raymond. 1966. Peace and War: A Theory of International Relations. Translated by Richard Howard and Annette Baker Fox. New York: Doubleday and Co.

Asare, Bossman E. 2007. "Federalism, Multi-level Governance and Tobacco

Control: Comparative Public Policy in the United Kingdom and South Africa" Dissertation submitted to the Eberly College of Art and Science. Morgantown: West Virginia University

Asare, Bossman. 2007. Tobacco Control - Comparative Public Policy in the United Kingdom and South Africa. Germany: VDM Verlag Dr. Muller.

Asare, Bossman. 2009. Tobacco Regulation in South Africa: Interest Groups and Public Policy. African Journal of Political Science and International Relations 3 (3):99-106

Asma, Samira and Linda Pederson, 1999. "Tobacco Control in Africa: Opportunities for Prevention” Tobacco Control 8(4): 353-354 
Assunta, M. 2004. Unfiltered: Conflict over Tobacco Policy and Public Health. Tobacco Control, 13, 455.

Ballard, John. 2004. “The Politics of Tobacco Control in Australia: International Template?” In Ronald Bayer and Eric A. Feldman (eds.) Unfiltered: Conflicts over Tobacco Policy and Public Health. Cambridge: Harvard University Press. pp 68-88

Baggott, Rob. 1988. "Health V. Wealth: The Politics of Smoking in Norway and the UK." Glasgow: Strathclyde Papers on Government and Politics No. 57, Department of Politics.

BAT. 1997. Consumer and Regulatory Affairs. http://legacy.library.ucsf.edu/tid/lpn63a99 Retrieved 11/26/2009

BAT. 1980. Africa Public Affairs Conference: The Changing Policies of WHO and their Implications for BAT Companies in Africa. http://legacy.library.ucsf.edu/tid/sgn26a99. Retrieved 1/ 21/2011

Barnum, Howard. 1994. The Economic Burden of Global Trade in Tobacco. Tobacco Control, 3, 358-361.

Barraclough, Simon and Martha Morrow. 2008. "A Grim contradiction: The Practice and Consequences of Corporate Social Responsibility by British American Tobacco in Malaysia” Social Science \& Medicine 66: 1784-1796

Bayer, Ronald and James Colgrove. 2004. "Children and Bystanders First: The Ethics and Politics of Tobacco Control in the United States". In Ronald Bayer and Eric A. Feldman (eds.) Unfiltered: Conflict over Tobacco Policy and Public Health Cambridge: Harvard University Press. Pp. 8-37 
Becker, Andrew. 2010. “American Cancer Society to Manage New Effort against Tobacco in Africa" American Cancer Society http://pressroom.cancer.org/index.php?s=43\&item=219 Retrieved 6/12/2010

Beyer, Joy de and Waverley, Brigden. 2003. Tobacco Control Policy Strategies, Success and Setbacks. Washington DC: The World Bank/RITC

Bennett, Colin J. 1991a. "How States Utilize Foreign Evidence” Journal of Public Policy 11:3154

Bennett, Colin J. 1991b. "What is Policy Convergence and What Causes it?" British Journal of Political Science 21:215-233

Berry, Frances S. 2008. "Innovators and Copycats: Path Dependency and Eight Other Models of Policy and Management Diffusion”. Paper Prepared for Presentation at the American Political Science Association Conference in Boston, MA August 28-31

Berry, Frances S and William D. Berry. 2007. "Innovation and Diffusion Models in Policy Research” In Paul Sabatier (ed.) Theories of the Policy Process. Colorado: Westview Press

Berry, Frances S and William D. Berry. 1999. "State Lottery Adoptions as Policy Innovation: An Events History Analysis”. American Political Science Review 84: 395-415

Berg, Bruce. 2004. Qualitative Research Methods for the Social Sciences. $5^{\text {th }}$ Ed. Boston: Allyn and Bacon.

Blanke, Douglas, and da Costa Silva (Eds). 2004. Tobacco Control Legislation: An Introductory Guide. World Health Organization. 
Bomberg, Elizabeth and Peterson, John M. 2000. Policy Transfer and Europeanization: Passing the Heineken Test? Paper for the Political Studies Association-UK 50th Annual Conference April 10-13 London

Borio, Gene. 2001. Tobacco BBS Web page: http://www.tobacco.org

Brenya, Edward and Bossman Asare. 2011. "Traditional Institutions and Agenda Setting: The Case of the Adoption of the Ghana Education Trust Fund" African Research Review, Volume 5 (3): 90-103

Cairney, Paul. 2007. “A ‘Multiple Lenses' Approach to Policy Change: The Case of Tobacco Policy in the UK" British Politics (2): 45-68

Cairney, Paul, Hadii Mamudu, and Donley T. Studlar. 2012. Global Tobacco Control Policy: Power, Policy, Governance and Transfer. London: Palgrave.

Camilleri, L. C., \& Morgan Stanley. 2009. Philip Morris International: Unpublished.

Camilleri, Louis C and Morgan Stanley. 2008. Remarks by the Chairman and CEO of Philip Morris International Inc. Global Consumer and Retail Conference November 18, 2008. http://www.pmi.com/eng/media_center/speeches_and_presentations/documents/FINAL_ 1118-800am_MS_DISTRIBUTION_REMARKS\%5B1\%5D.pdf

Canadian Global Tobacco Control Forum 2006 "Strengthening Global Tobacco Control: Evaluative Report" Health Bridge Canada. Retrieved in 7/15/11 from http://www.healthbridge.ca/CGTCFevaluativereport_may17_.pdf

Caporaso, James. 1997 'Across the Great Divide: Integrating Comparative and International Politics', International Studies Quarterly (41): 563-92

Castles, Francis (Ed.). 1993. Families of Nations: Patterns of Public Policy in Western Democracies. Aldershot: Dartmouth 
Centers for Disease Control and Prevention (CDC). 1999. Best Practices for

Comprehensive Tobacco Control Programs. Atlanta, GA: U.S. Department of

Health and Human Services, Centers for Disease Control and Prevention, National

Center for Chronic Disease Prevention and Health Promotion, Office on Smoking and

Health.

Chapman, S., \& Fahey, P. 1989. Cigarette smoking and economic prosperity: the relationship of GNP per capita and cigarette consumption in 103 countries. Sydney, Australia:

Department of Community Medicine, University of Sydney

Chitanondh, H. 2001. Defeat in Trade-Victory in Health. Bangkok, Thailand: Thailand Health

Promotion Institute and The National Health Foundation

Collin, J., LeGresley, E., MacKenzie, R., Lawrence, S., \& Lee, K. 2004. Complicity in

Contraband: British American Tobacco and Cigarette Smuggling in Asia. Tobacco

Control, 13, 104-111

Collin, Jeff, Kelley Lee, and Karen Bissell. 2002. "The Framework Convention on Tobacco Control: The Politics of Global Health Governance" Third World Quarterly 23: 265-282

Collier, David and Richard .E. Messick. 1975. "Prerequisites versus Diffusion: Testing Explanation of Social Security Adoption”. American Political Science Review 69:12991315

Common, Richard, 2001. Public Management and Policy Transfer in Southeast Asia. Burlington: Ashgate Publishing Company

Commonwealth Secretariat 2011 "Progress Report: Commonwealth Secretariat Road Map on Non-Communicable Diseases" Commonwealth Secretariat Retrieved on 
$7 / 15 / 11$ from

http://www.thecommonwealth.org/files/236498/FileName/HMM(G)(11)4Update_on_ Commonwealth_Road_Map.pdf

Cook, Brain J. 1996. Bureaucracy and Self-Government. Baltimore: The John Hopkins University Press

Crain, R.L. 1966. “Fluoridation: “The Diffusion of Innovation Among Cities”. Social Forces 44:467-476

Creswell, John. 2001. Research Design: Qualitative and Quantitative Approaches, $2^{\text {nd }} \quad$ Ed. Thousand Oaks, CA: Sage Publications.

Derthick, Martha. 2002. Up in Smoke: From Legislation to Litigation in Tobacco Politics. Washington, D.C.: CQ Press.

Diani Mario. 2000. "Social Movement Networks Virtual and Real” Information, Communication \& Society (3) 3: 386-402

Dolowitz, David P. 2003. “A Policy-makers Guide to Policy Transfer” Political Science Quarterly 74(1): 101-108

Dolowitz, David P. 1998. Learning from America: Policy Transfer and the Development of the British Workfare State. Brighton: Sussex Academic Press

Dolowitz, David P. 1997. "British Employment Policy in the 1980s: Learning from the American Experience" Governance 10: 23-42

Dolowitz, David P. and D. Marsh. 1996. "Who Learns What from Whom: A Review of Policy Transfer Literature" Political Studies 44; 343-357

Dolowitz, David. P. and David Marsh. 2000. "Learning from Abroad: The Role of Policy Transfer in Contemporary Policy-Making.” Governance 13(1):5-23 
Dolowitz, David P, Stephen Greenwold, and David Marsh. 1999. "Something Old, Something New, Something Borrowed, But Why Red, White and Blue?” Parliamentary Affairs 52 (4): 719-730

Drezner, Daniel W. 2001. “Globalization and Policy Convergence” International Studies Review 3(1): 53-78

Drope, Jeffrey. 2010. “The Politics of Smoke-free Policies in Developing in Developing Countries: Lessons from Africa” CVD Prevention and Control (5):65-73

Efroymson, Debra and Ahmed Saifuddin. 2003. "Building Momentum for Tobacco Control: The Case of Bangladesh”. In Joy de Beyer and Linda Waverley Brigden (ed.). Tobacco Control Policy: Strategies, Successes and Setbacks. Washington DC: The World Bank/RITC

Edwards, Kasper. 2001. "Epistemic Communities, Situated Learning and Open Source Software Development" Paper Prepared for the Epistemic Cultures and the Practice of Interdisciplinarity’ Workshop NTNU, Trondheim, June 11-12 www.Epistemic_com_Sit_Learn and OSS v07.doc (Retrieved 8/27/2009)

Eldring, Line, Sabata Nakayanye, and Malehoko Tshoaedi. 2000. "Child Labor in Tobacco Growing Sector in Africa" Report prepared for the IUF/ITGA/BAT Conference on the Elimination of Child Labor, Nairobi 8-9th October 2000

Ellis, GA; Hobart RL and Reed DF. 1996. “Overcoming a Powerful Tobacco Lobby in Enacting Local Smoking Ordinances: The Contra Costa County Experience” Journal of Public Health Policy 17:28-46

Eriksen, Michael, Judith Mackay, and Hana Ross. 2012. The Tobacco Atlas, (Fourth Edition). American Cancer Society

Evans, Mark. 2004 (Ed). Policy Transfer in Global Perspective. Burlington: Ashgate: 


\section{Publishing Company}

Evans, Mark and Jonathan Davies. 1999. “Understanding Policy Transfer: A Multi-Level, MultiDisciplinary Perspective" Public Administration Vol. 77(2): 361-385

Fakhfakh, Radhouane, Mohamed Hsairi, Mohsen Maalej, Nourredinne Achour, and Taoufik Nacef. 2002. "Tobacco Use in Tunisia: Behavior and Awareness.” Bulletin of World Health Organization, 80:350-375.

Farquharson, Karen. 2003. "Influencing Policy Transnationally: Pro-and Anti-Tobacco Global Advocacy Networks.” Australian Journal of Public Administration, 62(4):80-92.

Farrell, Bryan. 2009. “Up in Smoke: Big Tobacco Ruins a Small African Nation” Earth Island Journal Winter Issue. Retrieved on 10/16/ 2011 from http://www.earthisland.org/journal/index.php/eij/article/up_in_smoke1

Feldman, Eric. 2004. "The Limits of Tolerance: Cigarettes, Politics, and Society in Japan.” In Ronald Bayer and Eric A. Feldman (eds.) Unfiltered: Conflicts over Tobacco Policy and Public Health, 38-67. Cambridge: Harvard University Press

Finnemore, Martha. 1996. National Interest in International Society. Ithaca: Cornell University Press

Flora, Meerjardy, C G N Mascie - Taylor, Mahmudu Rahman. 2009. “Gender and Locality Difference in Tobacco Prevalence among Adult Bangladesh” Tobacco Control 18: 445450

Food and Agriculture Organization. 2003. Projections of Tobacco Production, Consumption and Trade to the Year 2010. Rome, Italy: Food and Agriculture Organization. 
Framework Convention Alliance. 2001. "FCTC Negotiations” Website:

http://fctc.org/archives/inbl.shtml (Retrieved 11/25/2007)

Framework Convention Alliance. 2007. “Annual Report on Tobacco Control for 2007”

http://www.fctc.org/index.php?option=com_docman\&task=doc_download\&gid=126\&Ite mid=76 (Retrieved on 7/5/10)

Frankenberg, Gunter. 2004. "Between Paternalism and Voluntarism: Tobacco Consumption and Tobacco Control in Germany. In Ronald Bayer and Eric A. Feldman (eds.) Unfiltered: Conflicts over Tobacco Policy and Public Health, 161-189. Cambridge: Harvard University Press.

Frey, Scott R. 1997. “The International Traffic in Tobacco” Third World Quarterly 18(2):303319

George, Alexander L and Andrew Bennett. 2005. Case Studies and Theory Development in the Social Sciences. Cambridge, M.A: MIT Press

Ghana News Agency “CEPS Destroys 330,000 Cartons of Cigarettes” Ghana News Agency Report, December 13, 2009

Giddens, Anthony. 1984. The Constitution of Society: Outline of the Theory of Structuration Berkeley: University of California Press

Gilmore, A. B., \& McKee, A. M. 2004. "Moving East: How the Transnational Tobacco Industry Gained Entry to the Emerging Markets of the Former Soviet Union-part I: Establishing Cigarette Imports”. Tobacco Control, 13, 143-150.

Glantz, A. Stanton, John Slade, Lisa A. Bero, Peter Hanauer, and Deborah E. Barnes. 1996. The Cigarette Papers. Berkeley: University of California Press. 
Global Smokefree Partnership. 2009. Global Voices Report: Rebutting the tobacco Industry, Winning Smokefree Air, 2009 Status Report. Naples, Italy: Pixel Entropy

Givel, Michael S and Stanton A Glantz. 2000. "Failure to Defend a Successful State Tobacco Control Program: Policy Lesson from Florida” American Journal of Public Health 90 Issue 5: $762-767$

Goldstein, Judith and Robert Keohane O (eds.) 1993 Ideas and Foreign Policy: Beliefs, Institutions, and Political Change. New York: Cornell University Press

Graham, Erin, Craig Volden, and Charles R. Shipan. 2008. "The Diffusion of Policy Diffusion" Paper Prepared for Presentation at the American Political Science Association Conference in Boston, MA August 27-31

Gray, Virginia. 1973. "Innovation in the States: A Diffusion Study.” American Political Science Review, 67: 1174-85.

Guevara, M. W. 2008. Tobacco Underground: The Booming Global Trade in Smuggled Tobacco. http://www.publicintegrity.org/investigations/tobacco/pages/introduction Retrieved 10/17/2009

Gyebi, Edmond “Ghana: Three Poorest Regions Lead in Cigarette Smoking,” Ghana Chronicle June 7, 2007, Accra

Haas, Ernest B. 1990. When Knowledge is Power: Three Model of Change in International Organization. Berkeley: University of California Press Haas, Peter. 1992. "Introduction: Epistemic Communities and International Policy Coordination," International Organization, 43(1):377-403

Haas, Peter. 1989. "Do Regimes Matter? Epistemic Communities and the Mediterranean Pollution Control” International Organization 46:1-35 
Hajat, Rafiq and Dyton Milanzi. 2008. “Analytical Presentation of the Second Meeting (Budget) of the $40^{\mathrm{TH}}$ Session of Parliament" Malawi National Assembly: Office of the Clerk of Parliamentary June $30^{\text {th }}$

Hall, Peter. 1993. "Policy Paradigms, Social Learning and the State" Comparative Politics 25: 275- 295

Hammond, R and M Assunta. 2003. "The Framework Convention on Tobacco Control: Promising Start, Uncertain Future. Tobacco Control; 12: 241-242 http://tobaccocontrol.bmj.com/cgi/content/full/12/3/241\#FN1 Retrieved on 11/29/2007

Hargrove, G. C., \& INFOTAB. 1981. Developing Countries Group. http://legacy.library.ucsf.edu/tid/ghr25e00 . Retrieved 11/ 22/ 2009.

Heclo Hugh. 1978. "Issue Networks and the Executive Establishment” In Anthony King (ed.) the New American Political Systems. Washington, D.C: American Enterprise Institute, pages $87-124$

Hirschhorn, N. 2006. Research Reports and Publications based on tobacco industry documents, 1991-2006. Retrieved 11/23/200 from http://tobacco.health.usyd.edu.au/site/gateway/docs/pdf/Hirschhorn_publications_list.pdf Hudson, Alan. 2001. 'NGOs’ Transnational Advocacy Networks: From ‘Legitimacy’ To 'Political Responsibility”' Global Networks 1(4): 331-52

INFOTAB. 1990. Key Activities \& Key Products. http://legacy.library.ucsf.edu/tid/yym40c00 . Retrieved 10/30/ 2009.

INFOTAB. 1987. “The Industry in Action” http://tobaccodocuments.org 
INFOTAB. 1985. "A General Briefing on INFOTAB" http://tobaccodocuments.org/ness/37888.html?zoom=750\&ocr_position=above_foramatt ed\&start_page=31 Retrieved 10/21/2009

INFOTAB. 1984. INFOTAB Board of Directors Meeting Brussels. http://legacy.library.ucsf.edu/tid/cxv75d00. Retrieved 10/20/2009

INFOTAB, \& Advisory Group. 1983. Following Discussion, the Chairman suggested that the Secretary General and the Advisory Group prepare an Action Plan to Progressively Improve the Quality of NMAs. http://legacy.library.ucsf.edu/tid/ptd88d00. Retrieved 2/12/2011

International Development Research Center. 2009. "Research for Action on FCTC: Report on the Second Round of a Small Grants Competition" Ottawa, Canada". http://www.idrc.ca/uploads/user-S/12514719831RITC_FCTC2_ENG-correctedFINAL.pdf. Retrieved on 7/7/2010

International Development Research Center (IDRC). No date. Governance, Equity and Health Website www.idrc.ca/geh Retrieved 11/25/2007

International Development Research Center (IDRC) 1995. Exporting the Epidemic: An International Health Disaster. Website http://www.idrc.cs/en/ev-1-201-1-DO TOPIC.html (Retrieved October 26, 2007)

Jacobs, Rowena, H. Frederick Gale, Thomas Copehart C. Ping Zhang and Prabhat Jha. 2000. "The Supply-side Effects of Tobacco Control Policies in Prabhat Jha and Frank Chaloupka (eds.) Tobacco Control in Developing Countries Oxford: Oxford University Press, pp 311-341 
Jaffee, Steven. 2003. "Malawi’s Tobacco Sector: Standing on One Strong Leg is Better than on none” Africa Region Working Paper Series No.55. Retrieved from http://www.worldbank.org/afr/wps/wp55.pdf on 5/25/2011

Jha, Prabhat, and Frank J. Chaloupka. 2000. “The Economics of Tobacco Control.” British Medical Journal, 321:358-361.

Jha, Prabhat and Frank Chaloupka (Eds.). 2000. Tobacco Control in Developing Countries (eds.), Oxford University Press

Jha, Prabhat and Frank J. Chaloupka (eds.). 1999. Curbing the Epidemic: Governments and the Economics of Tobacco Control. Washington, D.C.: World Bank.

Joossens, Lau and Raw M. 2006. "The Tobacco Control Scale: A New Scale to Measure Country Activity" Tobacco Control 15:241-253

Jordan, Lisa and Tuijl, Peter V. 2000. "Political Responsibility in Transnational NGO Advocacy" World Development (28) 12: 2051-2065

Joy Online “Tobacco Control Bill Scrapped” Joy News Monday January 10, 2011

Kagan, Robert A. and David Vogel. 1993. “The Politics of Smoking Regulation: Canada, France, and the United States.” In Robert L. Rabin and Stephen D. Sugarman (eds.), Smoking Policy: Law, Politics, and Culture, New York: Oxford University Press.

Kahneman, Daniel and Amos Tversky 1984. “Choices, Values and Frames” American Psychologist 39: 341-50

Kaiser, Karl. 1971. "Transnational Politics: Towards a Theory of Multinational Politics". International Organization 25:790-817 
Kapito, John. 2000. Testimony Offered For a strong and binding Framework Convention on tobacco Control (FCTC) at the Public Hearing Organized by the World Health Organization (WHO) in Geneva, October 12 and 13

Keck, Margaret E and Kathryn Sikkink.1998. Activists beyond Borders: Advocacy Networks in International Politics. Ithaca: Cornell University Press

Keck, Margaret E and Kathryn Sikkink. 1999. "Transnational Advocacy Network in International and Regional Politics” International Social Science Journal (51) 159: 89101

Keohane, Robert O and Joseph Nye. 1972. Transnational Relations and World Politics. Cambridge: Harvard University Press

Keohane, Robert O. 1989. International Institutions and States Power: Essays in International Relation Theory. Boulder: West view Press

Khan, MM, Aklimunnessa K, Kabir MA, Kabir M, and Mori M. 2006. "Tobacco Consumption and its Association with Drug Use among Men in Bangladesh" Addiction 101(8):117886.

King, Gary, Robert Keohane O, and Sidney Verba. 1994. Designing Social Inquiry: Scientific Inferences in Qualitative Research. Princeton, New Jersey: Princeton University Press

Kingson, Jeorge 2010. “We Need a Tobacco Control Law” January 21, 2010. Retrieved from www.ghanweb.com/GhanaHomepage/features / on 1/22/2010

Krasner, Stephen D. 1983. International Regimes Ithaca: Cornell University Press

Krishnan, P, Margaret Mungherera, and Sinéad B Jones. 2003. "Tobacco and the Commonwealth: A Call to Action" The Lancet 361: May 17. Retrieved from on 2/19/ 2010 from www.thelancet.com 
Ladi, Stella (ed.). 2005. Globalization, Policy Transfer and Policy Research Institutes. MA: Northampton, Edward Elgar.

Lando, Harry A, Belinda Borrelli, Laura C. Klein, Linda P. Waverley, Frances A. Stillman, Jon D. Kassel, and Kenneth E. Warner . 2005. "The Landscape in Global Tobacco Control: A Guide to Gaining a Foothold” American Journal of Public Health 95(6): 93945

Latif, Ehsan. 2004. "Stubbing It Out” A Briefing for Commonwealth Health Ministers on How to Implement the Framework Convention on Tobacco Control, Commonwealth Policy Unit Laugesen, Murray, Michelle Scollo, David Sweanor, Saul Shiffman, Joe Gitchell, Kathy Barnsley, Mark Jacobs, Gary A Giovino, Stanton Glantz, Richard A Daynard, Gregory N Conolly, and Joseph R Difranza. 2000. "World Best Practices in Tobacco Control" Tobacco Control Journal 9(2): 228-236. Retrieved on 9/28/2009 from website http://tobaccocontrol.bmj.com/cgi/reprint/9/2/228

LeGresley, E., Lee, K., Muggli, M. E., Patel, P., Collin, J., and Hurt, R. D. 2008. British American Tobacco and the "insidious impact of illicit trade" in cigarettes across Africa. Tobacco Control, 17, 339-346.

Leichter, Howard M. 1983. "The Pattern and Origins of Policy Diffusion: The Case of the Commonwealth" Comparative Politics 15(2): 223-233

Leonard Zahn \& Associates. 1981. Informational Memorandum International Union Against Cancer (UICC). http://legacy.library.ucsf.edu/tid/gyo88c00 . Retrieved 11/18/2009. Licari, Michael J., and Kenneth J. Meier. 1997. "Regulatory Policy When Behavior is Addictive: Smoking, Cigarette Taxes and Bootlegging." Political Research Quarterly, 50:5-24. 
Lopez, Allan D, Neil E. Collishaw, and Tapani Piha. 1994. "A Descriptive Model of the Cigarette Epidemic in Developed Countries”. Tobacco Control 3(3): 242-247

Mackay, J., Eriksen, M., \& Shafey, O. 2006. The Tobacco Atlas (2nd ed.). Atlanta, GA: American Cancer Society

Makoka, Donald, Munthali, K., and Drope, Jeffrey. M. 2011. "Tobacco Control in Malawi”, in Drope, Jeffrey. M. (Ed). Tobacco Control in Africa - People, Politics and Policies, International Development Research Centre (IDRC), Ottawa.

Malan, Mia and Rosemary Leaver .2003. "Political Change in South Africa: New Tobacco Control and Public Health Policies" In Joy de Beyer and Linda Waverley Brigden (Eds.). Tobacco Control Policy: Strategies, Successes and Setbacks. Washington DC: The World Bank/RITC

Mamudu, Hadii M. 2005. "The Politics of Tobacco Control: The Formation and Function of the Framework Convention on Tobacco Control (FCTC)". Dissertation submitted to the Eberly College of Art and Science. Morgantown: West Virginia University.

Mamudu, Hadii M. and Stanton A. Glantz. 2009. "Civil Society and the Negotiation of the Framework Convention on Tobacco Control.” Global Public Health, 4:150-168

Mamudu, H.M., and D Studlar. 2009. "Multilevel Governance and Shared Sovereignty: The European Union, Member States, and the Framework Convention on Tobacco Control" Governance; 22(1):73-97

Mamudu, Hadii M., Ross Hammond, and Stanton A. Glantz. 2008. “Tobacco Industry Attempts to Counter World Bank Curbing the Epidemic and Obstruct the WHO Framework Convention on Tobacco Control.” Social Science and Medicine; 67(11): $1690-9$ 
Mamudu, H.M., Hammond, R., and S.A. Glantz. 2008. “'Project Cerberus': Tobacco Industry Strategy to Create an Alternative to the Framework Convention on Tobacco Control" American Journal of Public Health 98: 1630-42

Mamudu, Hadii M., Ross Hammond, and Stanton A. Glantz. "International Trade vs. Public Health Issue during the FCTC Negotiations, 1999-2003.” American Journal of Public Health [Submitted)

Manfredi, Christopher and Antonia Maioni. 2004. "Rights and Public Health in the Balance: Tobacco Control in Canada.” In Ronald Bayer and Eric A. Feldman (eds.) Unfiltered: Conflicts over Tobacco Policy and Public Health. Cambridge: Harvard University Press, pp. 68-88

Manheim, Jarol B, Richard Rich C, Lars Willnat, and Craig Leonard Brians. 2006. Empirical Political Analysis: Research Methods in Political Science. New York: Pearson Education Inc.

Marmor T. R. and Lieberman E. S. 2004. "Tobacco Control in Comparative Perspective: Eight Nations in Search of an Explanation.” In Ronald Bayer and Eric A. Feldman (eds.) Unfiltered: Conflicts over Tobacco Policy and Public Health, 275-291. Cambridge: Harvard University Press

McAdam, D, McCarthy J, Zald M (eds). 1996. Comparative Perspectives on Social Movements: Political Opportunities, Mobilizing Structures, and Cultural Framings. New York: Cambridge

McNamara, Kathleen L. 1998. The Currency of Ideas: Monetary Politics in the European Union. New York: Cornell University Press 
Merriam, Sharan B. 1998. Qualitative Research and Case Study Application in Education. San Franscisco, CA: Jossey- Bass Publishers

Ministry of Health, Ghana Health Service, and Framework Convention on Tobacco Control. 2010. 'Needs Assessment for Implementation of the WHO Framework Convention on Tobacco Control in Ghana" Report to the FCTC

Minstrom, Michael and Karen Mossberger. 2008. "The Politics of Diffusion and the Diffusion of Policy Innovation" Paper Prepared for Delivery at the American Political Science Association Conference in Boston, MA August 28-31

Minstrom, Michael. 1997. "Policy Entrepreneurs and Diffusion of Innovation” American Journal of Political Science 42 (3): 738-70

Munthali, Kondwani. 2009. "Update of Malawi Tobacco Control Efforts and Appeal for Renewed Support for Legal and Advocacy Campaigns" Tobacco Control in Africa Retrieved 10/16/2010 from http://blogsofbainbridge.typepad.com/africa/2010/01/malawilawsuit-against-a-tobacco-control-advocate.html "Law Suit against Tobacco Control Activist" Tobacco Control in Africa Retrieved 10/16/2010 from http://blogsofbainbridge.typepad.com/africa/2010/01/malawi-lawsuit-against-a-tobaccocontrol-advocate.html

“Tobacco Company Handover School worth USD \$ 130,000” Tobacco Control in Africa Retrieved 10/16/2010 from http://blogsofbainbridge.typepad.com/africa/2010/01/malawi-lawsuit-against-a-tobaccocontrol-advocate.html 
"Malawi Government to Sign FCTC to Fight Tougher Tobacco Control Legislations" Tobacco Control in Africa Retrieved 10/16/2010 from http://blogsofbainbridge.typepad.com/africa/2010/01/malawi-lawsuit-against-a-tobaccocontrol-advocate.html

"YASED Launches Stay Healthy and Tell a Friend Ribbon Campaign” Tobacco Control in Africa Retrieved 10/16/2010 from http://blogsofbainbridge.typepad.com/africa/2010/01/malawi-lawsuit-against-a-tobaccocontrol-advocate.html "Research Indicates Malawians Will Support Smoking Advertising Ban” Tobacco Control in Africa Retrieved 10/16/2010 from http://blogsofbainbridge.typepad.com/africa/2010/01/malawi-lawsuit-against-a-tobaccocontrol-advocate.html

Nadelmann, Ethan A. 1990. "Global Prohibition Regimes: The Evolution of Norms in International Society," International Organization, 44: 479-526.

Nathan, Rose. 2004. Model Legislation for Tobacco Control: A Policy Development and Legislative Drafting Manuel $\left(1^{\text {st }} \mathrm{Ed}\right)$. France: International Union for Health Promotion and Education (IUHPE).

Nathanson, Constance A. 1999. "Social Movements as Catalysts for Policy Change: The Case of Smoking and Guns”. Journal of Health Politics, Policy and Law 24:421- 488

Nelson, Bettey Naa. 2009. "VALD Selected to Participate in Global Summit" Ghana Chronicle August $24^{\text {th }}$

Nelson, Thomas E, Zoe M. Oxley, and Rosalee A Clawson. 1997. “Toward A Psychology of Framing Effects” Political Behavior 19 (3): 221- 46 
Newmark Adam J. 2002. "An Integrated Approach to Policy Transfer and Diffusion” The Review of Policy Research 19(2): 151-178

Newmark Adam J. 1999. "Diffusion of Uniform Programs Throughout School Districts in Florida" Paper Presented at the Annual Meeting of Political Studies Association Nottingham. England, March 23-25

Novotny, T.E, Mamudu, H.M. 2008. Progression of Tobacco Control Policies Implications for Global Action and for Tobacco Control in Developing Countries. Washington, D.C: World Bank

Nye, Joseph S and Robert O Keohane. 1971. "Transnational Relations and World Politics: A Conclusion" International Organization 25(3):721-748

Observer. 1985. UICC DEVELOPMENTS. OBSERVER'S REPORT. http://legacy.library.ucsf.edu/tid/hcj44e00 . Retrieved 11/18/2009.

Odoi-Larbi, Stephen. 2009. "Parliament to Ban Tobacco Use in Public" Ghana Chronicle June 3 rd

Oppong-Anane, Kwame. 2001. Country Pasture: Ghana Retrieved on 6/31/10 from http://www.fao.org/ag/AGP/agpc/doc/Counprof/Ghana/Ghana.htm

Opukah, Shabanji. 1996. Amesca Meeting Monday 29th January 1996: CORA Report. http://legacy.library.ucsf.edu/tid/olh61a99 . Retrieved 11/22/2009

O'Sullivan, B., and Chapman, S. 2000. "Eyes on the Price: Transnational Tobacco Companies in China 1976-1997”. Tobacco Control, 9, 292-302.

Otanez, M.G., Mamudu H.M., and S.A. Glantz. 2009. “Tobacco Companies Use Malawi to Lobby Against Global Tobacco Control.” American Journal of Public Health 99(10): 1759-1771 
Otanez, M.G., Mamudu H., and S. A. Glantz. 2007. "Global Leaf Companies Control the Tobacco Market in Malawi.” Tobacco Control 16, 261-9

Otanez, M G, Muggli M E, and S. A. Glantz. 2006. "Eliminating Child Labor in Malawi: A British American Tobacco Corporate Responsibility Project to Sidestep Tobacco Labour Exploitation" Tobacco Control 15:224-2

Owusu-Dabo, Ellis, Ann McNeil, Sarah Lewis, Anna Gilmore, and John Britton .2010. "Status of Implementation of Framework Convention on Tobacco Control (FCTC) in Ghana: A Qualitative Study” BioMed Central Public Health 10 (1) 1-11. http://www.biomedcentral.com/1471-2458/10/1 Retrieved 7/3/10

Owusu-Dabo, Ellis, Sarah Lewis A, Anna Gilmore, and John Britton .2009. "Smoking Uptake and Prevalence in Ghana" Tobacco Control 18 (5): 365-70

Pampel, Fred. 2008. “Tobacco Use in sub-Sahara Africa: Estimates from The Demographic Health Surveys” Social Science and Medicine. 2008 April; 66(8): 1772-1783.

Pan American Health Organization (PAHO). 2003. The Framework Convention for Tobacco Control: Strengthening Health Globally. Website http://www.paho.org/English/DD/PUB/tobacco.pdf (Retrieved October, 26, 2007)

Panos Institute. 1994. Tobacco: Health and Beyond - Developing Countries at the Crossroads. Retrieved from http://legacy.library.ucsf.edu/tid/zye17a99 on June 24, 2011.

Patton, Michael Quinn. 2002. Qualitative Research and Evaluation Methods. 3rd Edition. Thousand Oaks, CA: SAGE publications

Peters, Guy 1998. Comparative Politics: Theories and Methods. New York: New York University Press

Peters, Guy. 1997. "The Diffusion of Administrative Reform” West European Politics (Autumn) 
Petracca, Mark, P. 1992. The Politics of Interests: Interest Groups Transformed. Colorado: West view Press

Philip Morris. 1993. The Activist Movement. http://legacy.library.ucsf.edu/tid/glg14e00. Retrieved 11/18/2009.

Philip Morris. 1981a. “Developing Countries Group Progress Report Covering Projects and Action Plans Since the Board of Directors Meeting on March 30, 1981. http://legacy.library.ucsf.edu/tid/dhr25e00 . 11/22/ 2009.

Philip Morris. 1981b. “The International 'Conspiracy' Against Tobacco”. http://legacy.library.ucsf.edu/tid/znw23e00 . 9/11/2009

Piper, Nicola and Andrea Uhlin .2004. "New Perspectives on Transnational Activism” in Nicola Piper and Andrea Uhlin (eds.) Transnational Activism in Asia: Problems of Power and Democracy. New York: Rutledge, pp. 1-25

Proctor, Robert N. 1999. The Nazi War on Cancer. Princeton: Princeton University Press Pross, A. Paul, and Ian S. Stewart. 1994. "Breaking the Habit: Attentive Publics and Tobacco Regulation.” In Susan Philips (ed.), How Ottawa Spends, 1994-1995, Ottawa: Carleton University Press

Public Health Association of South Africa .2007. Leadership in Tobacco Control for Public Health Associations in East and Southern Africa. Phasa Newsletter Issue 9. Website http://www.phasa.za.org/newsletter/sep07/index.html (Retrieved October 26, 2007)

Rafiq Hajat and Dyton Milanzi .2008. "Analytical Presentation of the Second Meeting (Budget) of the $40^{\text {th }}$ Session of Parliament (Budget)" National Assembly: Office of Clerk of Parliament, Malawi

Read, Melvyn. 1996. The Politics of Tobacco: Policy Networks and the Cigarette Industry. Aldershot, Hampshire: Avebury. 
Research for International Tobacco Control. 2006. Setting Tobacco Control Priorities for East, Central and Southern Africa Region International Development Research Center, Canada's website publication: http://www.idrc.ca/en/ev-84117-201-1-DO_TOPIC.html (Retrieved June 1, 2009)

Risse-Kappen, Thomas. (ed.) .1995. Bringing Transnational Relations Back in Non State Actors, Domestic Structure and International Institutions. Cambridge: Cambridge University Press

Robertson, David Brain. 1991. "Political Conflict and Lesson Drawing” Journal of Public Policy $11: 55-78$

Roemer, Ruth. 1982. Legislative Action to Combat the World Smoking Epidemic. Geneva: World Health Organization.

Roemer, Ruth. 1993. "Legislative Action to Combat World Tobacco Epidemic” Geneva: WHO

Roemer, Ruth and Allyn L Taylor .2005. "Origins of the WHO Framework Convention on Tobacco Control” American Journal of Public Health 95(6): 936-938

Rogers, Everett M.1995. Diffusion of Innovations. New York: The Free Press

Rose, Richard. 2005. Learning from Comparative Public Policy: A Practical Guide. New York: Rutledge

Rose, Richard 1991. "What is Lesson Drawing?” Journal of Public Policy 11:3-30

Rubin, Herbert J. and Irene S. Rubin. 1995. Qualitative Interviewing. Thousand Oaks, CA: Sage Publications

Sabatier, Paul and Hank Jenkins-Smith. 1999. “The Advocacy Coalition Framework.” In Paul A. Sabatier (ed.), Theories of the Policy Process, Boulder: West view Press. 
Saloojee, Yussuf. 1993a. “Tobacco Control: A Case Study (South Africa).” In Proceedings of All Africa Conference on Tobacco and Health. November 1 1993, Harare, Zimbabwe.

Samuels Bruce and Stanton A Glantz .1991. "The Politics of Local Tobacco Control” Journal of the American Medical Association 266:2110-17

Schneider, Anne and Helen Ingram. 1998. "Systematically Pinching Ideas: A Comparative Approach to Policy Design”. Journal of Public Policy 8:61-80

Schreiner, Bruce and Emery P. Dalesio. 2008. "10 Years after Settlement, Tobacco Rebounds in US" Yahoo News November 25. Associated Press

Sebenius James K. 1992. “Challenging Conventional Explanations of International Cooperation: Negotiation Analysis and the Case of Epistemic Communities" International Organization 46(1):323-65

Seeliger, Robert. 1996. “Conceptualizing and Researching Policy Convergence” Policy Studies Journal 24 (2), 287-306

Selin H, and Bols M. 2002. Developing Legislation on Tobacco Control: Template and Guidelines. Washington, DC: Pan American Health Organization (PAHO)

Semu-Banda, Pilirani .2007. “Over One Million of Malawi’s Children Are Child Laborers” The WIP Retrieved 10/16/2009 from http://thewip.net/contributors/2007/08/over_one_million_of_malawis_ch.html\#more

Shafey, O., Eriksen, M., Ross, H., and Mackay, J. 2009. The Tobacco Atlas (3rd ed.). Atlanta, GA: America Cancer Society, World Lung Foundation

Shimkhada, Riti and John W. Peabody. 2003. "Tobacco Control in India.” Bulletin of the World Health Organization, 81:41-60 
Sikkink, Kathryn. 2002. "Transnational advocacy networks and the social construction of legal rules” In Yves Dezalay, and Bryant G. Garth (eds.) Global prescriptions: the production, exportation, and importation of a new legal orthodoxy. Ann Arbor: University of Michigan Press

Simpson, B., \& INFOTAB. 1985. International Scope and Major new Issues, Latin American Workshop, Miami, 30-31 July, 1985. http://legacy.library.ucsf.edu/tid/gjq52f00. Retrievd January 21, 2011

Simmons, Beth and Zachary Elkins. 2004. "The Globalization of Liberation: Policy Diffusion in the International Political Economy," American Political Science Review 98(1): 471- 492

Soerojo, Widyastuti and Bundiantoro Setyo .2007. "Indonesia Report Card: Status of Tobacco Use and its Control," Southeast Asia Tobacco Control Alliance (SEATCA)

Sklair, Leslie. 2002. "The Transnational Capitalist Class and Global Politics: Deconstructing the Corporate-State Connection” International Political Science Review 23(2): 159-174

Slaughter, Anne-Marie. 2004. A New World Order. Princeton, New Jersey: Princeton University Press

Spill, Rorie, Michael Licari, and Leonard Ray. 2001. “Taking on Tobacco: Policy Entrepreneurship and Tobacco Litigation.” Political Research Quarterly, 54:605-622

Statistics, Research and Information Directorate (SRID). 2001. Agriculture in Ghana. Facts and Figures. Ministry of Food and Agriculture. Accra. Ghana

Stone, Diane. 2004 "Transfer Agents and Global Networks in the Transnationalisation of Policy" Journal of European Public Policy 11: 545-566 
Studlar, Donley T .2007. "Ideas, Institution and Diffusion: What Explains Tobacco Policies in Australia, Canada and New Zealand". Commonwealth and Comparative Politics. 45(2):164-184

Studlar, Donley T. 2005. “The Political Dynamics of Tobacco Control in Australia and New Zealand: Explaining Policy Problems, Instruments, and Pattern of Adoption" Australia Journal of Political Science. 40:255-274

Studlar, Donley T. 2004a. "Lateral, Top-Down, or Bottom Up Policy Diffusion? Comparing Tobacco Control in the United States, Canada and Australia."

Studlar, Donley T. 2004b. "Tobacco Control Policy Instruments in a Shrinking World: How much policy learning?” In D. Levi-Faur and E. Vigoda-Gadot (eds.) International Public Policy and Management, 189-209. Marcel Decker

Studlar, Donley T. 2003. “Tobacco Control” In Leslie Pal and Kent Weaver (eds.), The Government Taketh Away: The Politics of Pain in the United States and Canada, 137166. Washington, DC: Georgetown University Press.

Studlar, Donley T. 2002b. Tobacco Control: Comparative Politics in the United States and Canada. Peterborough, Ontario: Broadview Press.

Studlar, Donley. 1999. "Diffusion of Tobacco Control in North America" Annals of the American Academy of Political and Social Science, Vol. 566, No.1, 68-79

Taylor, Peter. 1985. The Smoking Ring: Tobacco, Money and Multinational Politics. Revised Edition. New York: New American Library.

Thorup, Larsen L. 2009. "Smoking bans, health and Science: A comparative study of policy networks and smoking policies in England, Germany and Denmark" Retrieved from the 
on 8/26/2009 Social Science Research Network Website:

http://papers.ssrn.com/sol3/papers.cfm?abstract_id=1462298

Traynor, MP, ME Begay and Stanton A Glantz. 1993. "New Tobacco Industry Strategy to Prevent Local Tobacco Control" Journal of the American Medical Association 270:479-86

Trubek David M., Jim Mosher \& Jeffrey S Rothstein .2000. “Transnationalism in the Regulation of Labor Relations: International Regimes and Transnational Advocacy Networks", Law and Social Inquiry (25) 4: 1187-1211

U.S. Department of Health and Human Services. 1992. Smoking and Health in the Americas. Atlanta, GA: The U.S. Department of Health and Human Services, Public Health Services, Centers for Disease Control, National Center for Chronic Disease Prevention and Health Promotion, Office of Smoking and Health. DHHS Publication No. (CDC) 928419.

Vision for Alternative Development. 2010. “Civil Society Shadow Report on Ghana's Implementation of the Framework Convention on Tobacco Control" FCTC Verkerk, H. G., \& INFOTAB. 1985. INFOTAB Regional Workshop - Kuala Lumpur May 1315, 1985 International Overview of Main Issues. http://legacy.library.ucsf.edu/tid/jjq52f00 . Retrieved December, 19, 2010

Vogel, David and Robert Kagan A (eds.) 2004. The Dynamics of Regulatory Change: How Globalization Affects National Regulatory Policies. Berkeley: University of California Press

Walker, Jack L. 1969. “The Diffusion of Innovation among the American States” American Political Science Review 63: 880- 900 
Warner, Kenneth E. 2000. "The Need for, and Value of, a Multi-Level Approach to Disease Prevention: The Case of Tobacco Control" in Smedley, Brian D and S Leonard Syme (eds.) Promoting Health: Intervention Strategies from Social and Behavioral Research. Washington, D.C.: National Academy Press

Warren, C. W., Asma, S., Lee, J., Lea, V., \& Mackay, J. 2009. Global Tobacco Surveillance System: The GTSS Atlas. Atlanta, GA: The US Centers for Disease Control and Prevention, World Lung Foundation.

Waverly, Linda. 2007. “Global Tobacco Control: Resources for International tobacco control research and policy development" A Paper Presented to the Executives of the Canada Tobacco Control Research Initiative and the International Development Research Center. Retrieved from the website of IDRC on 10/16/2009: www.idrc.ca/ritc

Waverly, Linda. 2007. "Tobacco as a Development Issue” A Presentation Made to the Research for International Tobacco Control and the International Development Research Center. Retrieved from the website of IDRC on 10/16/2009: www.idrc.ca/ritc

Wellington, Edith; John Gyapong, Sophia Twum-Barima, Moses Aikins, and John Briton 2011 "Tobacco Control in Ghana" in Drope, Jeffrey.M. (Ed). Tobacco Control in Africa People, Politics and Policies, International Development Research Centre (IDRC), Ottawa.

Wellington, Edith K .2007. "Tobacco Control Effort in Ghana: Drafting of Tobacco Control Bill and Plan of Action for Tobacco Control" A Paper Presented at the Plenary Session of the Workshop for Research for Action on the FCTC 
Wellington, Edith K, Jane Amponsah, William Bosu, and Agyeman B. Akosa 2009 "Survey of the Implementation of the Framework Convention on Tobacco Control (FCTC) in Ghana" Ghana Health Service - Health Research Unit

Wendt, Alexander. 1992. “Anarchy Is What States Make of it- The Social Construction of Power- Politics” International Organization 46:391-425

White, Vicki. 2009. "Trend in the Prevalence of Smoking” in Scollo, Michelle and Margaret Winstanley (eds.) Tobacco in Australia: Facts and Issues. A Comprehensive Online Resource. Retrieved on 10/16/2009 from Website: http://www.tobaccoinaustralia.org.au/

Wolman, Harold. 1992. "Understanding Cross-National Policy Transfer: The Case of Britain and U.S” GOV. 5: 27-45

World Bank. 1999. Curbing the Epidemic: Government and Economics of Tobacco Control Washington, DC: World Bank

World Bank Report 1995

World Conference on Tobacco or Health (WCTOH) .2000. “World's Best Practices in Tobacco Control." Tobacco Control 9:228-236.

World Conference on Tobacco or Health (WCTOH). 1994. ( $8^{\text {th }}: 1992$ : Buenos Aires, Argentina). Building a Tobacco-Free World Atlanta, GA: American Cancer Society.

World Conference on Tobacco or Health (WCTOH). 1967. Toward a Less Harmful Cigarette (Proceedings) edited by Ernest L Wynder and Dietrich Hoffman of the National Cancer Institute. New York: New York

World Health Organization. 2009. History of the WHO Framework Convention on Tabaco Control Geneva: WHO Framework Convention on Tobacco Control 
World Health Organization. 2008. WHO Report on the Global Tobacco Epidemic: Mpower Package. Geneva: WHO

World Health Organization. 2005. WHO Collaboration Centers for Tobacco Control Geneva: WHO Press.

World Health Organization. 2004. "International Consultation on Environmental Tobacco Smoke (ETS) and Child Health Background Papers" Website: http://www.who, int/tobacco/fctc/consultation/ets2/en/ (Retrieved 11/26/2007)

World Health Organization. 2004. Scientific Advisory Committee on Tobacco Production Regulation. Retrieved on June 26, 2011 from http://www.who.int/tobacco/sactob/en/

World Bank. 2003. Economics of Tobacco Control in Developing World: A Background Paper for the High Level Round Table on Tobacco Control and Development

World Health Organization. 1999. The World Health Report 1999. Geneva: World Health Organization

World Health Organization. 1997. The World Health Report: Conquering Suffering, Enriching Humanity. Geneva: WHO. Website: http://www.who.int/whr/1997/media_centre/en/index.html

White, Anna. 1997. Cigarette Marketing in Senegal, West Africa. Tobacco Control, 6, 243-245. WHO- Framework Convention on Tobacco Control 2009 Overview of Control Measures Website http://www.who.int/fctc/text_download/en/ (Retrieved 7/12/09)

WHO: Framework Convention on Tobacco Control: Conference of Party Report, Ghana. 2007, 2-6-2009 
WHO Regional Office for Africa. 2000. Partners Unite on Tobacco Control Policy and Programming. Website http://www.afro.who.int/index.html (Retrieved October 26, 2007)

WHO Regional Office for Africa. 2007. "WHO, CDC to Accelerate Survey Aimed at Preventing Tobacco Related Death”. WHO -AFRO Website http://www.afro.who.int/index.html (Retrieved October 26, 2007)

Wolfson, Mark. 2001. The Fight against Big Tobacco New York: Aldine De Gruyter World Health Organization Regional Office for Eastern Mediterranean. 2001. Workshop on Media and Tobacco Enhancing the Role of the Media in Tobacco Control EMRO, Cairo, Egypt, 31 May - 3 June 2001. Website http://www.emro.who.int/tfi/tfi.htm (Retrieved October 26, 2007).

World Health Organization Regional Office for Eastern Mediterranean. 2007 "Tobacco Use in Shisha: Studies on Waterpipe Smoking in Egypt" WHO Regional Office for the Eastern Mediterranean/Egyptian Smoking Prevention Research Institute Website: www.emro.who.int/tfi/pdf/shisha.pdf. Retrieved on 10/16/2009

World Health Organization -Office for Eastern Mediterranean. 2004. Tobacco Industry Activities in the Eastern Mediterranean Region: Illicit Tobacco Trade. http://www.emro.who.int/TFI/pdf/TFI-019.pdf Retrieved 10/16/ 2009

World Health Organization Tobacco Free Initiative (TFI) Report 2007 World Health Organization. 1988e. WHO Programme on Tobacco or Health. http://legacy.library.ucsf.edu/tid/hsd00a99. Retrieved on July 10, 2010 
World Health Organization. 2005. WHO Collaborating Centers for Tobacco Control. Retrieved on July 10, 2010, from http://www.who.int/collaboratingcentres/networks/networksdetails/en/index 9. html

Wilson, Duff. 2010 “Gates Foundation Stops Grant to Canadian Research Center” New York Times Online April 12. Retrieve 4/24/2010 http://www.nytimes.com/2010/04/13/business/global/13gates.html?_r=1

Yach, Derek and Douglass Bettcher. 2000. "Globalization of the Tobacco Industry Influence and New Global Responses” Tobacco Control 9: 206-216

Youssef, R.M., S.A. Abou-Khatwa, and H.M. Fouad. 2002. "Prevalence of Smoking and Age of Initiation in Alexandria, Egypt” Eastern Mediterranean Health Journal 8 (4\&5).

Yunus, Mohammad. 2001. “Craving for Nicotine: A Study on Tobacco Prevalence in Bangladesh" A Report Presented to the World Health Organization. Retrieved from website:http://www.searo.who.int/LinkFiles/Regional_Tobacco_Surveillance_System_Se ntinelBangladesh.pdf 